Old Dominion University

ODU Digital Commons

Mechanical \& Aerospace Engineering Theses \&

Dissertations

Mechanical \& Aerospace Engineering

Spring 2020

\title{
A Study of the Aeroacoustics of Swept Propellers for Small Unmanned Aerial Vehicles
}

Arthur David Wiedemann

Old Dominion University, wiedemannarthur@gmail.com

Follow this and additional works at: https://digitalcommons.odu.edu/mae_etds

Part of the Acoustics, Dynamics, and Controls Commons, and the Aerospace Engineering Commons

\section{Recommended Citation}

Wiedemann, Arthur D.. "A Study of the Aeroacoustics of Swept Propellers for Small Unmanned Aerial Vehicles" (2020). Master of Science (MS), Thesis, Mechanical \& Aerospace Engineering, Old Dominion University, DOI: 10.25777/8qwv-vq29

https://digitalcommons.odu.edu/mae_etds/309

This Thesis is brought to you for free and open access by the Mechanical \& Aerospace Engineering at ODU Digital Commons. It has been accepted for inclusion in Mechanical \& Aerospace Engineering Theses \& Dissertations by an authorized administrator of ODU Digital Commons. For more information, please contact digitalcommons@odu.edu. 


\title{
A STUDY OF THE AEROACOUSTICS OF SWEPT PROPELLERS FOR SMALL UNMANNED AERIAL VEHICLES
}

\author{
by \\ Arthur David Wiedemann, \\ Old Dominion University \\ A Thesis Submitted to the Faculty of \\ Old Dominion University in Partial Fulfillment of the Requirements for the \\ Degree of \\ MASTER OF SCIENCE \\ AEROSPACE ENGINEERING \\ OLD DOMINION UNIVERSITY \\ May 2020
}

Approved By:

Colin Britcher (Director)

Drew Landman (Member)

Oktay Baysal (Member) 


\title{
ABSTRACT \\ A STUDY OF THE AEROACOUSTICS OF SWEPT PROPELLERS FOR SMALL UNMANNED AERIAL VEHICLES
}

\author{
Arthur David Wiedemann \\ Old Dominion University, 2020 \\ Director: Colin Britcher
}

In recent years, small unmanned aerial vehicles (UAVs) have increased in availability and use in both public and commercial sectors. This increased use of small UAVs or "drones" has the unfortunate consequence of introducing excessive noise into communities where they operate. Implementation of noise reduction methods is necessary if we wish to see expanded use of drones in public areas. With electric propulsion, the primary source of noise is the rotor/propeller used in contemporary multi-rotor configurations. In this thesis, the aerodynamics and acoustic behavior of various swept propellers is examined with computational and experimental methods.

A family of propeller geometries was established, with test articles machined from aluminum. A straight $16 \times 11.363$-inch propeller was used as a control for the nonconventional 15,35 , and 55 degree swept propellers. These propellers have a backwards sweeping blade centerline designed to "de-phase" the far-field acoustic pressure. The aerodynamic properties were collected in ODU's low speed wind tunnel and an acoustic experiment was conducted outdoors to determine the noise characteristics of the propeller while hovering. The computational analysis was done with NASA's Aircraft Noise Prediction Program 2 (ANOPP2). This program used the Farrassat-1A Formulation to integrate and propagate acoustic pressures generated by the propellers to observers in the far field at various RPM and distances for several propeller shapes. Overall, the aerodynamic efficiency of the non-conventional propellers did not sway from the conventional, straight propeller design. However, the dBA produced by each propeller requires further study, as the differences in sound pressure level between the designs could not be determined due to external environmental factors. 
Copyright, 2020, by Arthur David Wiedemann, All Rights Reserved. 


\section{ACKNOWLEDGMENTS}

During my time working on the thesis I have received guidance and aid from several people. First and foremost, I would like to thank my advisor, Dr. Colin Britcher, for his guidance and support. This thesis would have simply not been possible without his insight and guidance. I would also like to thank Dr. Drew Landman, who provided aid and feedback on my experiments, and Dr. Oktay Baysal for being on my committee. I must extend my gratitude to Larson Lindberg, from ODU's machine shop, who spent many hours advising me and working on the $\mathrm{CNC}$ machine to bring the propellers to life. My gratitude also goes out to Dr. Christopher Fuller, who provided the acoustic equipment needed to conduct the outdoor experiment. I am indebted to Dr. Leonard Lopes and the NASA ANOPP2 development team who designed and provided a fantastic simulation program for aeroacoustics. This program has been essential to my work. I would like to thank Engin Baris for dedicating his time and effort with programming and troubleshooting the LabView code used for the indoor and outdoor experiment. Lastly, I would like to thank my parents, Samantha Hahn, and Andrew Hahn for their support. 


\section{NOMENCLATURE}

\begin{tabular}{|c|c|}
\hline$p^{\prime}$ & Acoustic pressure, $\mathrm{Pa}$ \\
\hline$p_{\text {ref }}^{\prime}$ & Reference pressure, $\mathrm{Pa}$ \\
\hline$p_{r m s}^{\prime}$ & Root-mean-square pressure, $\mathrm{Pa}$ \\
\hline $\mathrm{R}$ & Radius of propeller \\
\hline $\mathrm{Ra}_{\mathrm{a}}$ & Radial position, in \\
\hline$C_{r}$ & Chord length, in \\
\hline$P$ & Pitch, in \\
\hline B & Geometric angle, deg \\
\hline$\Phi_{\#}$ & Phase angle, deg \\
\hline$\phi$ & Swept angle, deg \\
\hline$\theta$ & Horizontal radiation angle, deg \\
\hline$\varphi$ & Vertical radiation angle, deg \\
\hline$A_{\#}$ & Amplitude \\
\hline V & Forward velocity, $\mathrm{m} / \mathrm{s}$ \\
\hline$V_{\text {inf }}-(x)$ & Airstream velocity, $\mathrm{m} / \mathrm{s}$ \\
\hline$v_{a}$ & Tangential velocity, $\mathrm{m} / \mathrm{s}$ \\
\hline$n$ & Revolutions per second, rad/s \\
\hline $\mathrm{J}$ & Advance ratio \\
\hline$C_{t}$ & Coefficient of thrust \\
\hline$C_{q}$ & Coefficient of torque \\
\hline
\end{tabular}




\begin{tabular}{|c|c|}
\hline$C_{p}$ & Coefficient of power \\
\hline$C_{1}$ & Coefficient of lift \\
\hline$C_{d}$ & Coefficient of drag \\
\hline$\eta$ & Efficiency \\
\hline W & Torque, $\mathrm{N}^{*} \mathrm{~m}$ \\
\hline $\mathrm{T}$ & Thrust, N \\
\hline$\rho$ & Fluid density, $\mathrm{kg} / \mathrm{m}^{\wedge} 3$ \\
\hline q & Source function \\
\hline$a_{n}$ & Number of airfoils \\
\hline$S_{n}$ & Segment of Propeller \\
\hline$\Phi_{v}$ & Local inflow angle \\
\hline $\mathrm{t}$ & Observer time, s \\
\hline$\tau$ & Retarded time, s \\
\hline$x$ & Source position, $\mathrm{m}$ \\
\hline y & Observer position, $\mathrm{m}$ \\
\hline $\mathrm{r}$ & Distance between the observer and source, $m$ \\
\hline c & Speed of sound, $\mathrm{m} / \mathrm{s}$ \\
\hline $\mathrm{Li}$ & Dipole source term \\
\hline Un & Monopole source terms \\
\hline M & Mach number \\
\hline $\mathrm{Mr}_{\mathrm{r}}$ & Mach vector \\
\hline$\square^{2}$ & Box operator \\
\hline
\end{tabular}


TABLE OF CONTENTS

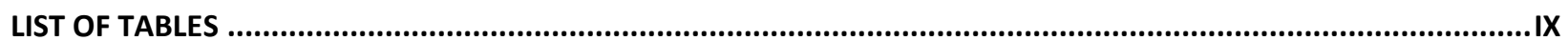

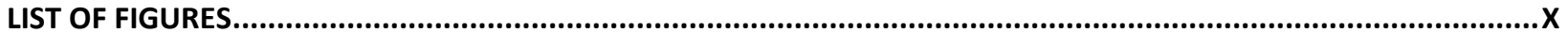

\section{CHAPTER}

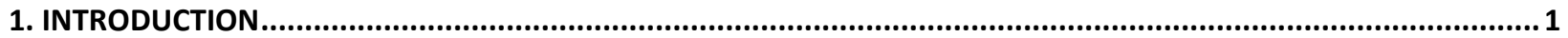

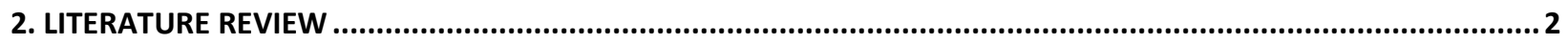

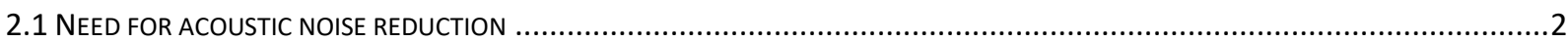

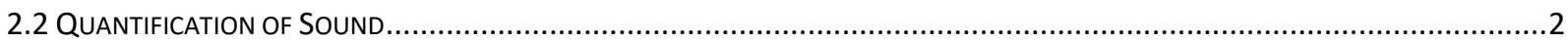

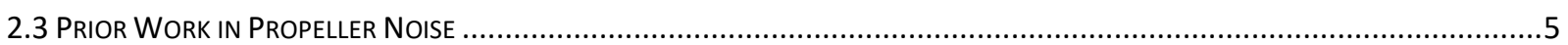

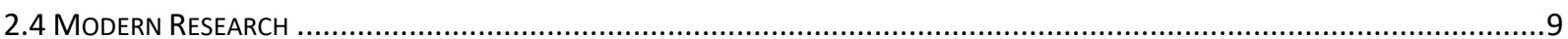

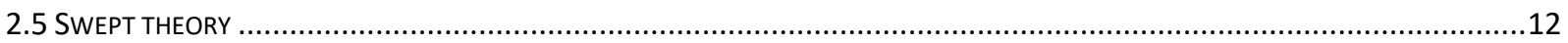

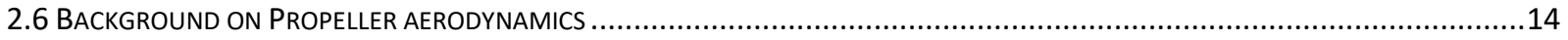

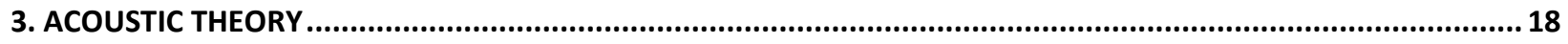

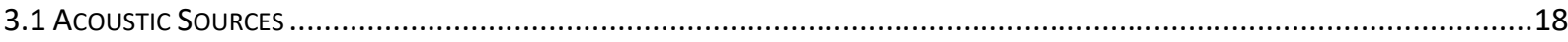

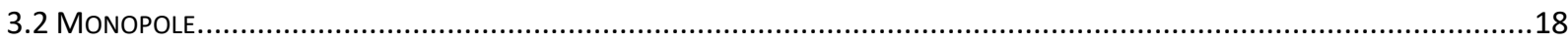

3.3 DIPOLE

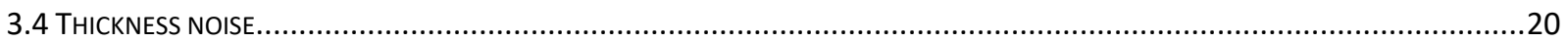

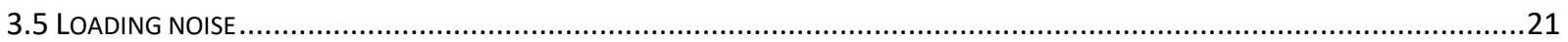

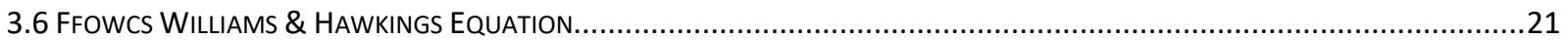

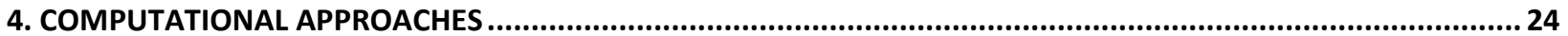

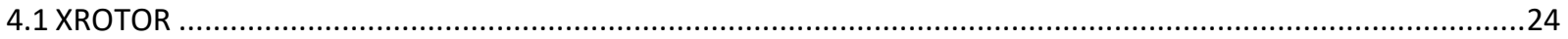

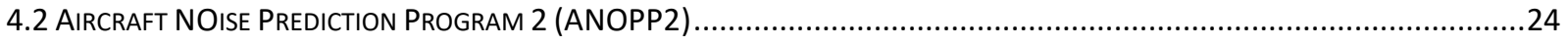

5. DEFINITION OF SWEPT PROPELLER AND MANUFACTURING ............................................................................26

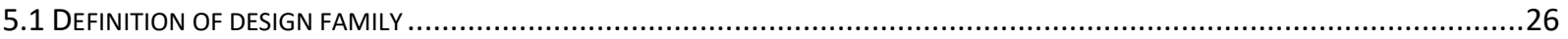

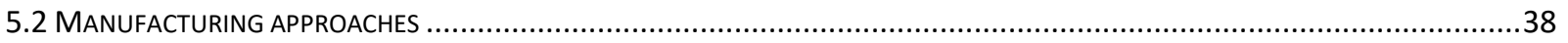

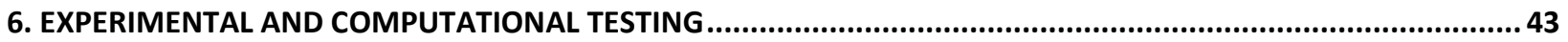

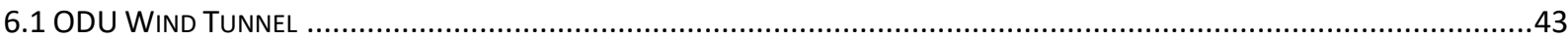

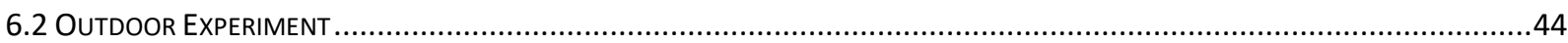

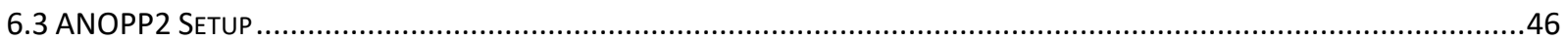

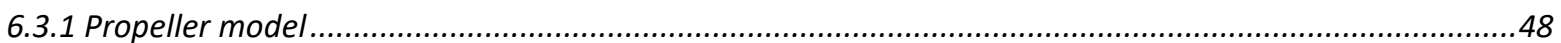

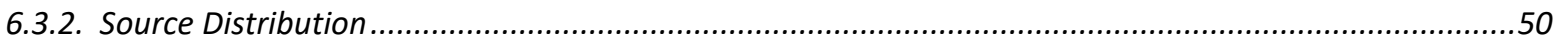

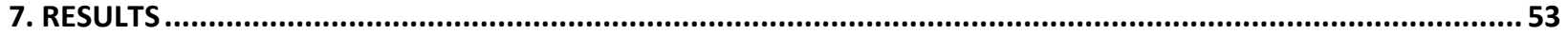

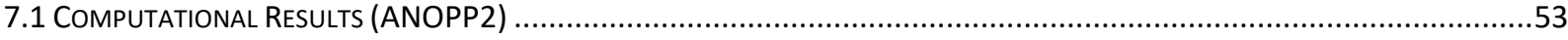

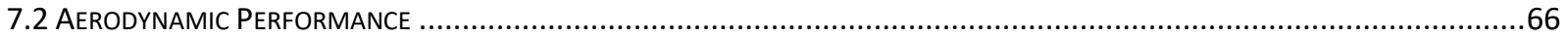

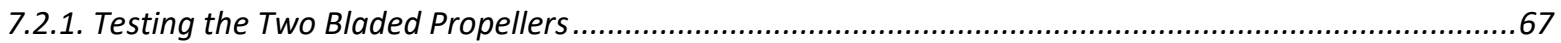

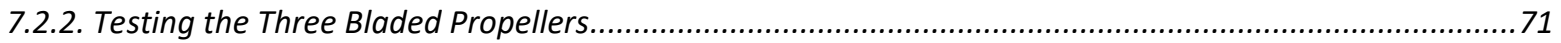

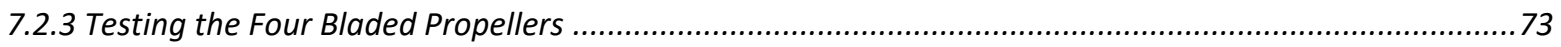

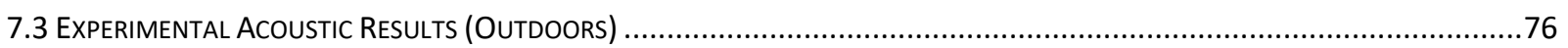

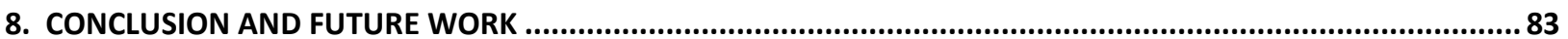




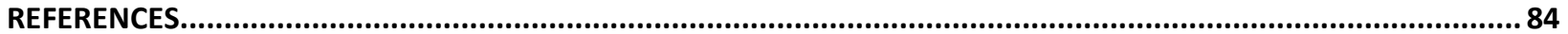

APPENDICES

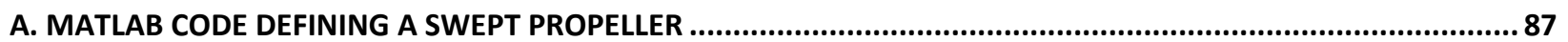

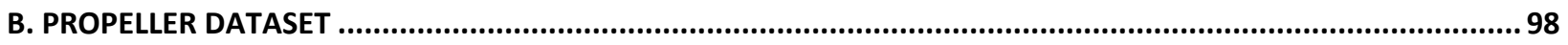

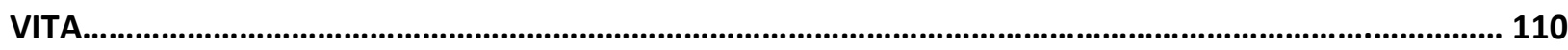




\section{LIST OF TABLES}

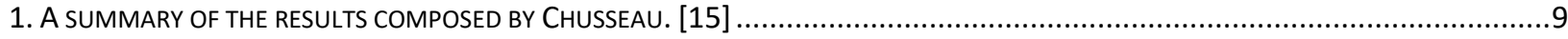

2. THE GEOMETRIC PROPERTIES USED TO DEFINE THE 3D SHAPE OF A TRADITIONAL PROPELLER. ...........................................15

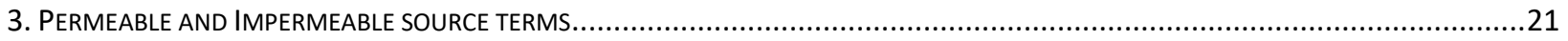

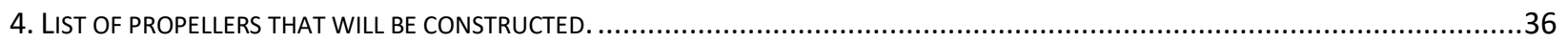

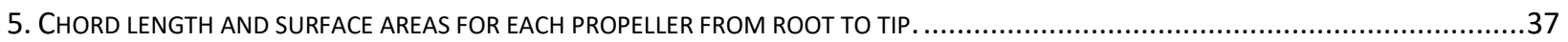

6. XROTOR DATA COMPARED TO EXPERIMENTAL DATA FOR THE STRAIGHT PROPELLER. ..........................................................49

7. XROTOR DATA COMPARED TO EXPERIMENTAL DATA FOR THE SWEPT-35 PROPELLER........................................................50

8. COMPARISON BETWEEN XROTOR AND EXPERIMENTAL VALUES FOR THE STRAIGHT PROPELLER 5000 RPM ...............................50

9. COMPARISON BETWEEN XROTOR AND EXPERIMENTAL VALUES FOR THE SWEPT-35 PROPELLER AT 5000 RPM...........................50

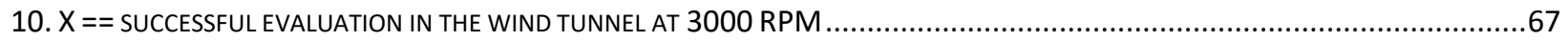

11. THE GEOMETRIC PROPERTIES USED TO DEFINE THE STRAIGHT PROPELLER (18 STATIONS DEFINED) .........................................98

12. THE GEOMETRIC PROPERTIES USED TO DEFINE THE SWEPT-15 PROPELLER (18 STATIONS DEFINED) .........................................98

13. THE GEOMETRIC PROPERTIES USED TO DEFINE THE SWEPT-35 PROPELLER (18 STATIONS DEFINED) .........................................99

14. THE GEOMETRIC PROPERTIES USED TO DEFINE THE SWEPT-55 PROPELLER (18 STATIONS DEFINED) .........................................99

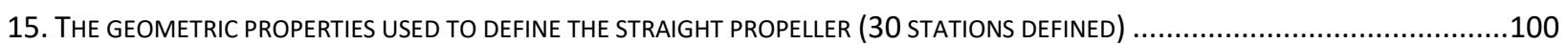

16. THE GEOMETRIC PROPERTIES USED TO DEFINE THE SWEPT-15 PROPELLER (30 STATIONS DEFINED) .......................................101

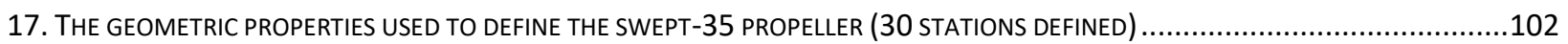

18. THE GEOMETRIC PROPERTIES USED TO DEFINE THE SWEPT-55 PROPELLER (30 STATIONS DEFINED) .....................................103 


\section{LIST OF FIGURES}

1. ESTIMATED NEW EQUAL LOUDNESS CONTOURS AND THE CONTOUR PROPOSED BY ROBINSON AND DADSON [5] ............................4

2. COMPARISON OF UNWEIGHTED AND A-WEIGHTED SPL VS FREQUENCY PLOT [6] .......................................................

3. SOUND-PRESSURE LEVEL MEASUREMENT RECORDED FROM FLYOVER AT 300 FEET BETWEEN THE UNMODIFIED AND MODIFIED TEST

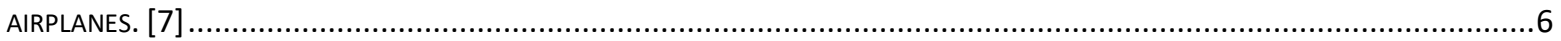

4A AND 4B: 4A SHOWS THE FOUR DIFFERENT TYPES OF ROTOR BLADES TESTED. 4B COMPARES THE SOUND-PRESSURE AGAINST THE TIP

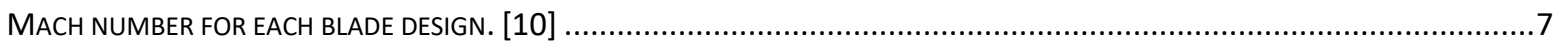

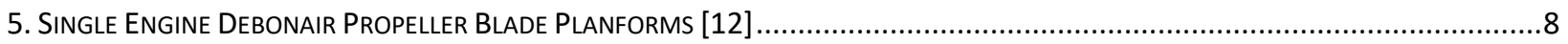

6A AND 6B: 6A SHOWS THE VARIOUS PROPFAN DESIGNS FROM REFERENCE 10. WHILE 6B SHOWS THE RESULT OF APPLYING THE SWEPT THEORY. THEY REPORTED A 5DB REDUCTION IN NOISE AND A SMALL INCREASE IN EFFICIENCY FOR THE SWEPT PROPELLER OVER THE

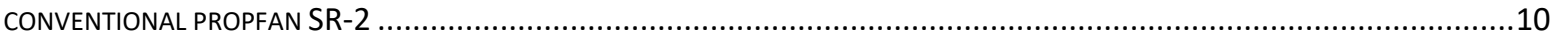

7. EXPERIMENTAL SETUP IN AN ANECHOIC CHAMBER TO EVALUATE THE ACOUSTIC NON-UNIFORM BLADE SPACING. [17] ....................10

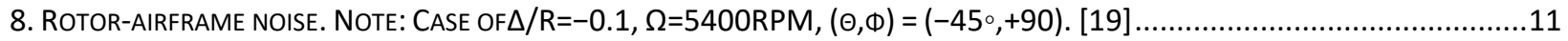

9. IMAGE AND GEOMETRIC DETAILS OF TESTED CONICAL AIRFRAME [19] ......................................................................12

10A \& 10B: 10A SHOWS THE COMPONENT CONTRIBUTION OF EACH AIRFOIL AND 9B SHOWS THE RESULTANT COMBINATION FROM THREE

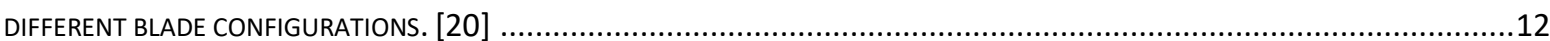

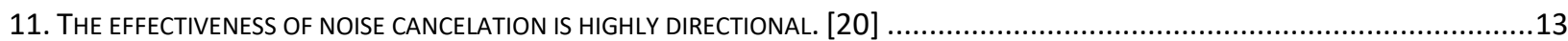

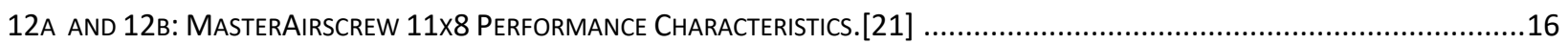

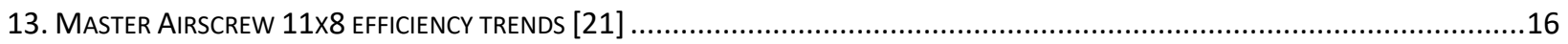

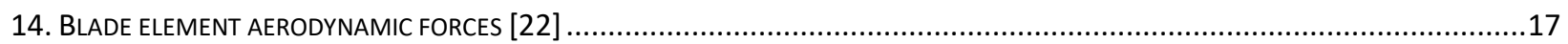

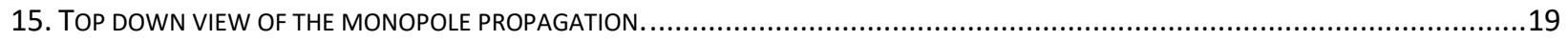

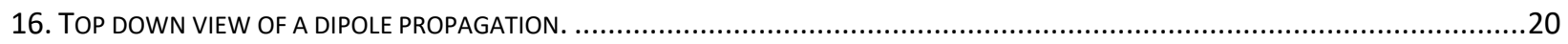

17.THE DEFINITION OF THE MOVING SURFACE AS $F(X, T)=0$. THIS SETS N AS THE UNIT OUTWARD NORMAL TO THE SURFACE. $F(X, T)>0$ AND

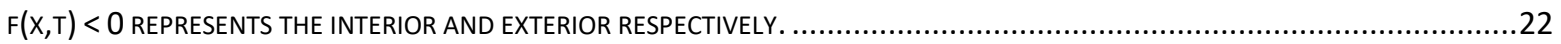

18. ANOPP2 INCORPORATES VARIOUS PHYSICAL EFFECTS INTO THE PHYSICS-BASED ANALYSIS. [30]........................................25

19. CONVENTIONAL TUBE-AND-WING AND UNCONVENTIONAL HYBRID WING BODY AIRCRAFT DESIGNS. [30] ...............................25

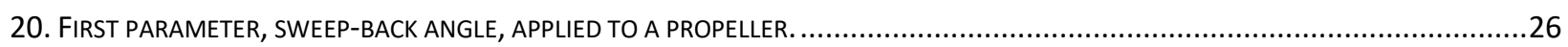

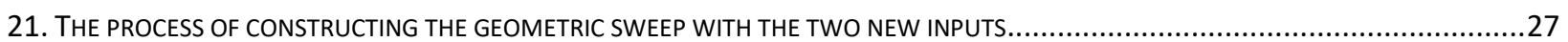

22. TOP-DOWN VIEW OF THE GEOMETRY FOR AN 8 -INCH PROPELLER WITH A 35-DEGREE SWEEP. THIS FIGURE PROVIDES AN ILLUSTRATION

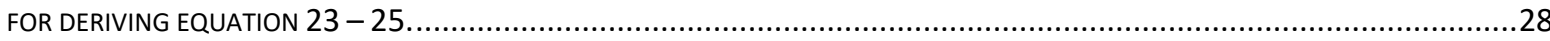

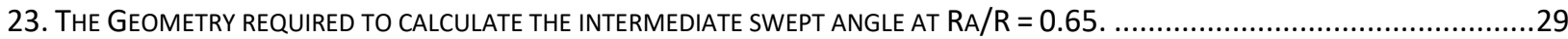

24A, 24B, 24C, AND 24D: THE DIFFERENCE BETWEEN SHEARING AND SWEEPING A PROPELLER BLADE. .........................................30

25A AND 25B: THE RELATIONS SHIP BETWEEN THE TANGENT SLOPE FOR THE AIRFOIL CHORD R/R $=0.65$, IN RED, AND THE CURRENT

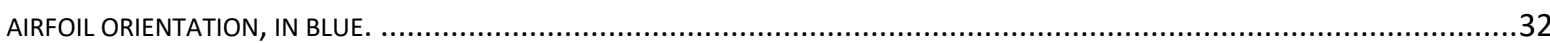

26A AND 26B: FINAL LAYOUT OF THE THREE-DIMENSIONAL $35^{\circ}$ SWEPT PROPELLER VIEWED FROM ABOVE AND THE SIDE.....................33

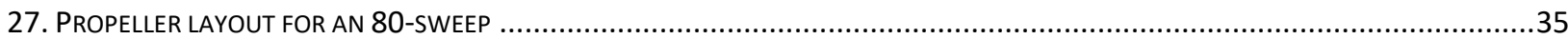

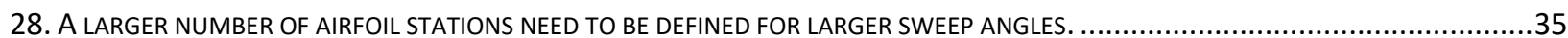

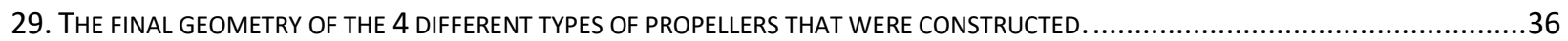

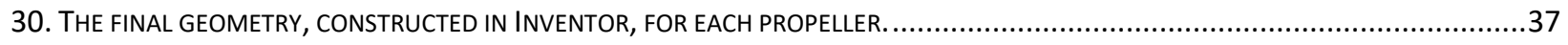

31.THE SUPER MINI MILL 2. THIS CNC MACHINE WAS USED TO MILL ALL 16 PROPELLERS......................................................38

32A \& 32B: 32A PRESENTS THE 1ST ROUGH CUT OF MILLING WITH THE 3DOF METHOD AND 32B PRESENTS THE 2 ND FINER CUT. ..........39

33. TWO SWEPT-35 PROPELLERS MADE IN THE 3DOF MILLER WITH THE TOP SIDE MILLED AWAY. THE HIGHLIGHTED PORTIONS WOULD BE

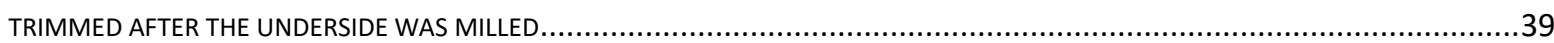

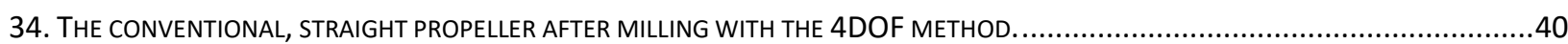

35. THE SWEPT-15 PROPELLER HELD IN WITH THE TAIL STOCK AND ROTARY TABLE FOR THE 4DOF MILLING METHOD........................41

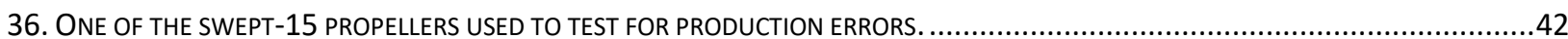

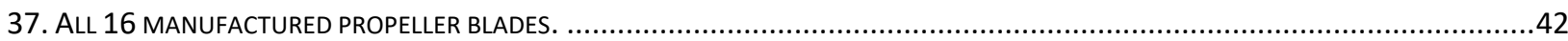

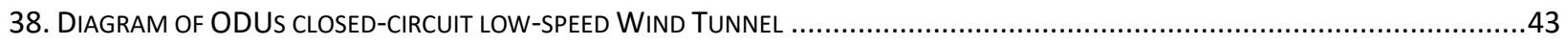




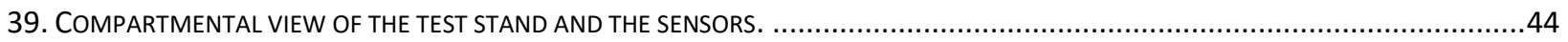

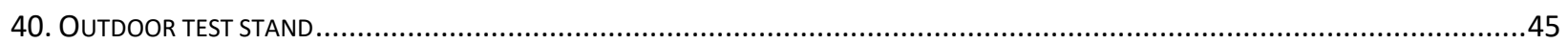

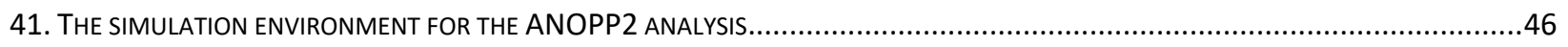

42. THE 50-METER SEMI SPHERE SHOWING THE SPHERICAL COORDINATE SYSTEM. ..............................................................47

43A. SHOWS THE APTH FILE COLLECTED FROM THE SIMULATION. 43B SHOWS THE FAR FIELD PRESSURE GRID AS A RESULT OF PLOTTING ALL PRESSURE WHEN TAU $=0.0045 \mathrm{~S}$.

44. COEFFICIENT OF LIFT AND DRAG ON THE PROPELLER. THE ARROWS SERVE AS A SKETCH AND NOT BASED OFF THE AERODYNAMIC

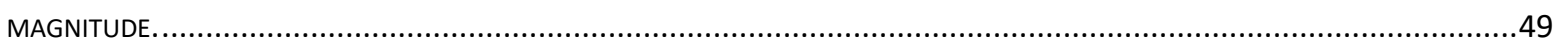

45. SKETCH OF THE PROPAGATION WAVES FROM THE PROPELLER TO OBSERVERS IN THE FAR-FIELD. .............................................51

46. SKETCH OF A MULTITUDE OF SOURCE PAIRS PROPAGATION TO THE FAR-FIELD AND THE SEGMENT DIVISION FOR THE PROPELLER. .......52

47. ANOPP2 CAN COMBINE MULTIPLE NOISE SOURCES AND PROPAGATE INFORMATION TO THE OBSERVER WITH ONE SURFACE. [30] ....52

48. THE STRAIGHT PROPELLERS GEOMETRY (TOP) AND ACOUSTIC PRESSURE OUTPUT (BELOW) FOR THE 7000 RPM AND 10M CASE.......54

49. THE SWEPT-35 PROPELLERS GEOMETRY (TOP) AND ACOUSTIC PRESSURE OUTPUT (BELOW) FOR THE 7000 RPM AND 10M CASE. ....54

50. THE SWEPT-55 PROPELLERS GEOMETRY (TOP) AND ACOUSTIC PRESSURE OUTPUT (BELOW) FOR THE 7000 RPM AND $10 M$ CASE. ....55

51. THE SWEPT-35 PROPELLER SPLIT IN MULTIPLE SOURCE POINTS ACROSS EACH BLADE. .........................................................56

52. TRENDS FOR THREE DIFFERENT PROPELLERS IN ANOPP2 AT VARIOUS RPM'S AND DISTANCES FOR THREE OBSERVER POINTS...........57

53. THE STRAIGHT PROPELLER'S ACOUSTIC PRESSURE AT VARIOUS RPMS RECORDED AT 10M FROM ALL ANGLES ON THE SEMISPHERE. ...58

54. THE SWEPT-35 PROPELLER'S ACOUSTIC PRESSURE AT VARIOUS RPM RECORDED AT 10M FROM ALL ANGLES ON THE SEMISPHERE. ...59

55. THE SWEPT-55 PROPELLER'S ACOUSTIC PRESSURE AT VARIOUS RPM RECORDED AT 10M FROM ALL ANGLES ON THE SEMISPHERE. ...60

56. DIPOLE AND MONOPOLE CONTRIBUTION TO THE TOTAL PRESSURE FOR THE STRAIGHT 2-BLADE PROPELLER AT 50M......................62

57. DIPOLE AND MONOPOLE CONTRIBUTION TO THE TOTAL PRESSURE FOR THE SWEPT-35, 2-BLADE PROPELLER AT 50M....................62

58. DIPOLE AND MONOPOLE CONTRIBUTION TO THE TOTAL PRESSURE FOR THE SWEPT-55, 2-BLADE PROPELLER AT 50M....................63

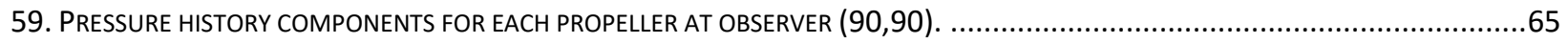

60. STRAIGHT AND SWEPT-55 COMPARED ACROSS VARIOUS BLADE COUNTS FROM OBSERVER $(90,90)$, 50M AWAY AND OPERATING AT AN

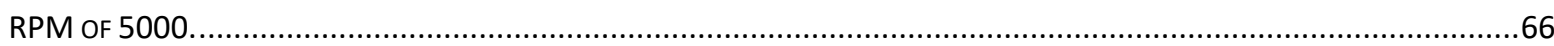

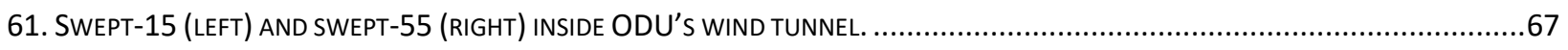

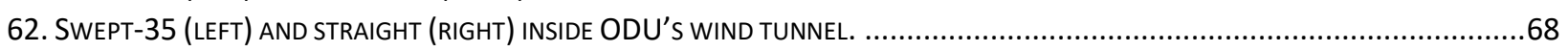

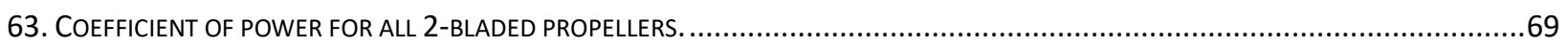

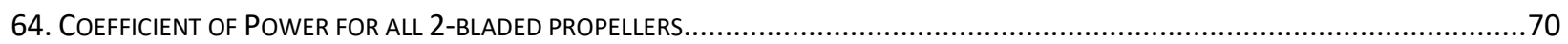

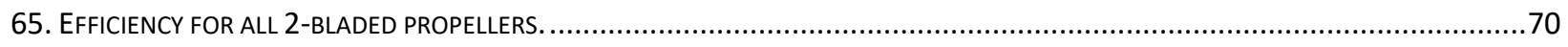

66. THE THREE DIFFERENT DESIGNS TESTED IN ODU'S WIND TUNNEL FOR THE THREE-BLADE CONFIGURATION. ...............................71

67. COEFFICIENT OF THRUST FOR THE STRAIGHT, SWEPT-35, AND SWEPT-55 3-BLADED PROPELLERS..........................................72

68. COEFFICIENT OF POWER FOR THE STRAIGHT, SWEPT-35, AND SWEPT-55 3-BLADED PROPELLERS. .........................................72

69. EFFICIENCY FOR THE STRAIGHT, SWEPT-35, AND SWEPT-55 3-BLADED PROPELLER ..........................................................73

70. THE TWO DIFFERENT DESIGNS TESTED IN ODU'S WIND TUNNEL FOR THE FOUR-BLADE CONFIGURATION..................................73

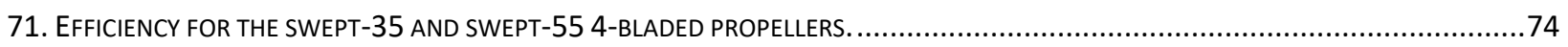

72. COEFFICIENT OF THRUST FOR THE SWEPT-35 AND SWEPT-55 4-BLADED PROPELLERS. ...................................................75

73. COEFFICIENT OF THRUST PLOT FOR THE SWEPT-35 AND SWEPT-55 4BLADED PROPELLERS. .................................................75

74. BACKGROUND NOISE AT VARIOUS POINT OF THE DAY. IT IS NOT NECESSARY TO PLOT THE NEGATIVE DB BUT A “WIDE VIEW" OF THE

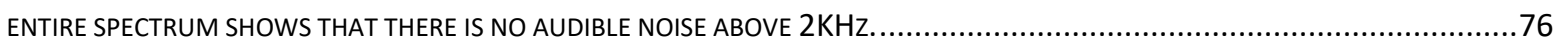

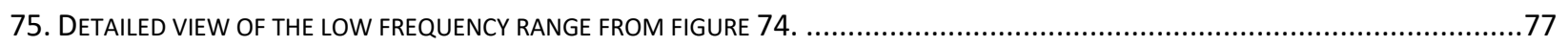

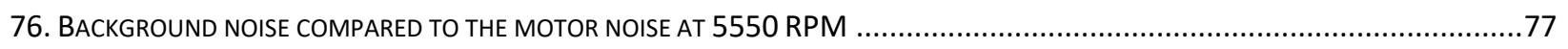

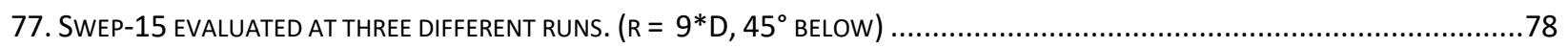

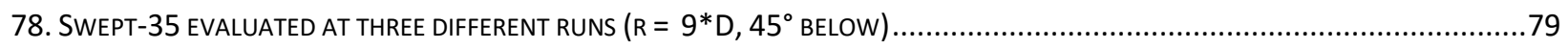

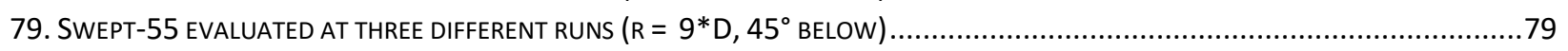

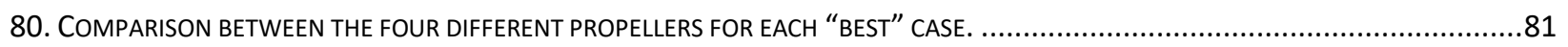

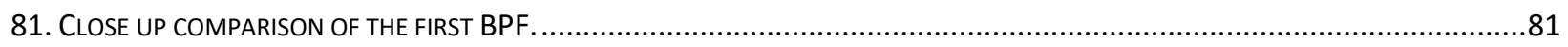

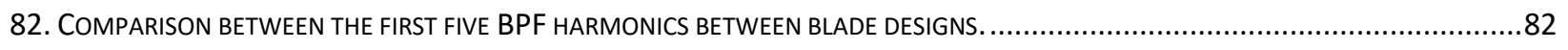

83. 'GEOMETRY.DAT' OUTPUT FROM THE MATLAB SCRIPT IMPORTED INTO TECPLOT .....................................................97 


\section{CHAPTER 1}

\section{INTRODUCTION}

Propellers provide an effective means of propulsion for submarines, cargo ships, unmanned aerial vehicles (UAV), and airplanes, where in all cases propellers are used to generate thrust. However, propellers often have the unfavorable effect of generating excessive noise. With air travel on the rise and the potential introduction of UAV's to ship goods and transport individuals, the skies are busier than ever before. With widespread use of UAV's, community noise would likely exceed what is deemed permissible by the Federal Aviation Administration (FAA). Previous studies have investigated methods of noise reduction for propellers such as lowering the rotational speed, reducing the radial length of the propeller blade, and adding more propellers to the rotorcraft (this makes up for the loss in thrust from slower, shorter blades). These methods have been used to reduce the total noise generated with the stipulation of reducing the thrust and increasing the weight. Additional research needs to be conducted on propeller noise to determine effective methods of noise reduction without degrading the aerodynamic efficiencies. Three augmented propeller blades and a control were evaluated both computationally and experimentally to determine the aerodynamic and acoustic effects of sweeping a propeller blade. The aerodynamics performance showed that the efficiencies did not suffer as a result of sweeping the blade for this propeller design, even at the most dramatic sweep. The acoustics were evaluated with both computation and experimental means. The computational analysis suggested that there was a slight increase as a result of swept propellers while the experimental showed lower noise. However, the acoustic experiment that was conducted in an outdoor setting suggests the swept propellers reduce noise. Additional testing is required to establish a definitive answer. 


\section{CHAPTER 2}

\section{LITERATURE REVIEW}

\subsection{NEED FOR ACOUSTIC NOISE REDUCTION}

Aircraft noise is an undesirable consequence of pressure fluctuations emitted by the airframe body and the propulsive devices as the aircraft moves through the medium . While there has always been some interest in noise reduction, the demand for noise reduction from commercial and governmental bodies has increased in recent years. This is likely due to the abundance of commercial and private aircraft along with the introduction of Unmanned Aerial Vehicles (UAVs) to the market. This has resulted in a busy and noisy sky.

The private sector has shown interest in applying small UAVs for commercial use. Small UAVs have sparked interest in shipping commodities, medical supplies, as well as agricultural applications to monitor crop growth and increase yield [1]. Other industries are investing in drone transportation, where these services would provide direct transportation between destinations. These innovations could provide tremendous benefit, but noisy drones could cause backlash from regulatory bodies if the community noise rose above acceptable levels.

\subsection{QUANTIFICATION OF SOUND}

Sound is a pressure fluctuation caused by a disturbance in the medium and is characterized by several properties such as frequency, sound pressure, speed of sound and direction. Discussion of direction will be covered in chapter 3 while the speed of sound, c, will be held as a constant. This leaves us with frequency and sound pressure to characterize and explore in this chapter.

Frequency is the number of repeating cycles over a duration of time. The human ear can detect sound waves from $20-20,000 \mathrm{~Hz}$ [2], although this range shrinks over the human lifespan. Frequency below $20 \mathrm{~Hz}$ is known as infrasound and above $20,000 \mathrm{~Hz}$ as ultrasound. The impact of frequency on human hearing will be discussed later, but it should be noted that lower frequency waves travel farther than higher frequencies. In addition, high frequency waves have less diffraction. This has resulted in applications for both humans and animals. Elephants use low frequency sound to communicate across vast distances. On the other end of the spectrum, bats use sonar in the ultrasound range, $20 \mathrm{kHz}-200$ $\mathrm{kHz}$, to locate prey in the dark [3]. It should be noted that bats can use low range frequencies to communicate as well. 
Sound pressure, also known as acoustic pressure, is the deviation from ambient pressure, $\mathrm{P}_{0}$, in the local pressure region due to an impulse caused by a disturbance. While acoustic pressure is measured in Pascals $(\mathrm{Pa})$ it is not the main term used to describe acoustic noise. The conventional term used to describe noise is the decibel, $\mathrm{dB}$. The decibel is a ratio between the acoustic pressure and a reference pressure (usually $20 \mu \mathrm{Pa}$ ) on a logarithmic scale. Equation (1) shows the relationship between the sound pressure and the logarithmic ratio, resulting in a metric known as the sound pressure level (SPL) with units in $\mathrm{dB}$. The log function is used because humans perceive sound intensity on a logarithmic rather than a linear relationship (Weber-Fechner law).

$$
d B=20 * \log _{10}\left(\frac{p^{\prime}}{p_{\text {ref }}}\right)
$$

One of the most fascinating graphs in acoustics is the equal-loudness contour, first constructed by Fletcher and Manson [4], which can be seen in figure 1. Fletcher and Manson exposed test subjects to pure tones at various frequencies over $10 \mathrm{~dB}$ increments. Listeners would report on an arbitrary frequency and $\mathrm{dB}$ level while simultaneously listening to a reference tone at $1000 \mathrm{~Hz}$. The arbitrary input was adjusted until the tones were perceived with identical loudness. Several listeners were tested and averaged to generate the trends with dashed lines. These lines indicated listeners perceived these noises as the same loudness, even when the actual sound-pressure-level varied. The results showed humans are significantly less sensitive to low-frequency noise $(20-800 \mathrm{~Hz})$ than medium or high frequencies. This indicates the human ear is not a perfect microphone that perceives all noises the same. However, pure tones are not directly applicable to noise experienced by humans [5], so the graph, while insightful, does not capture the complete picture of noise perception. Their work has been revised by the International Organization for Standards (ISO), and those improvements are represented by the solid lines. 


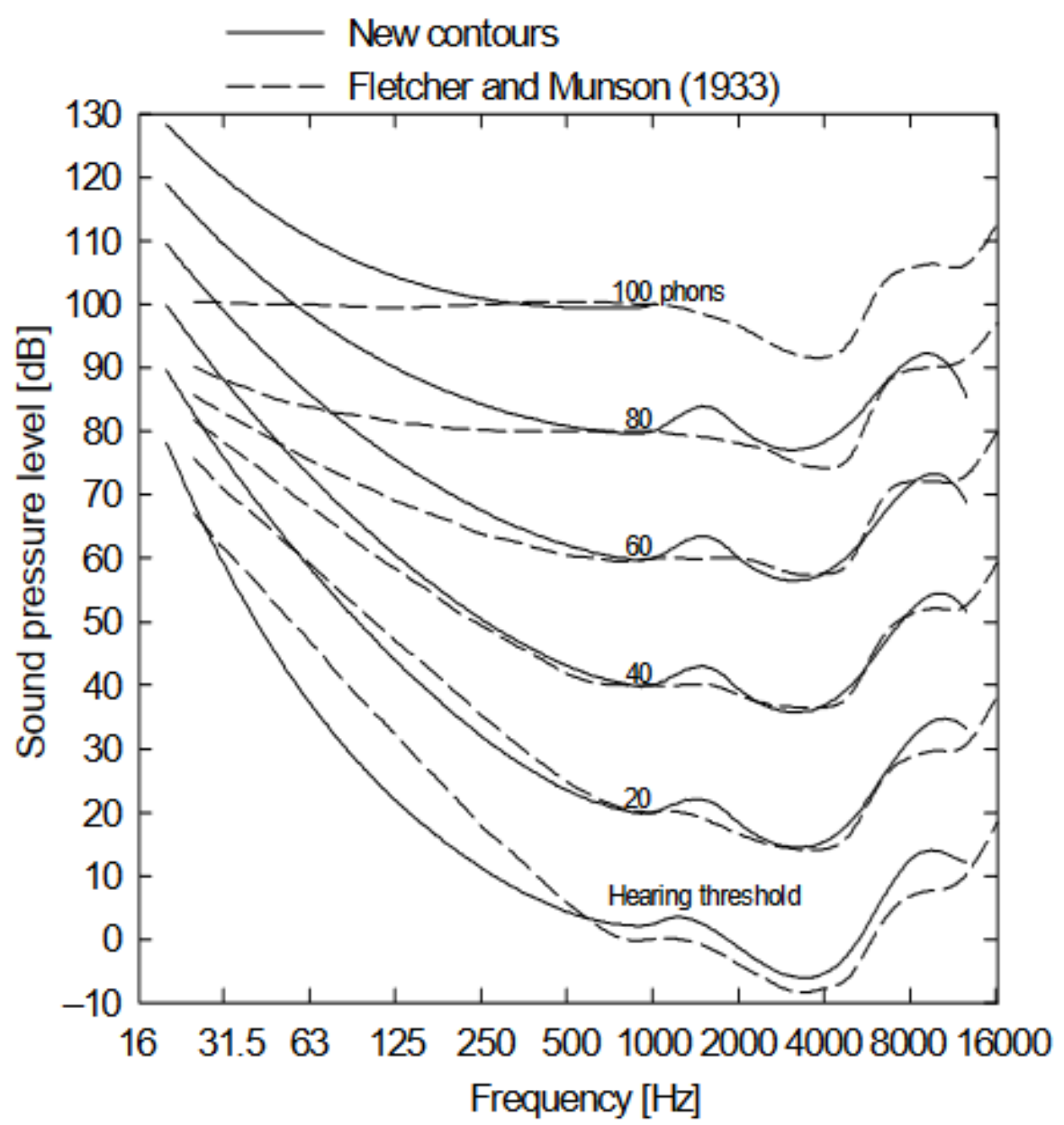

Figure 1: Estimated new equal loudness contours and the contour proposed by Robinson and Dadson [5]

These results also show the need to adjust the SPL from the actual acoustic signature to a weighted curve to better represent what humans perceive rather the pure noise itself. This is called the Aweighted curve and is defined by the international standard IEC which is a commonly used metric to evaluated environmental and industrial noise. Previous work conducted at Old Dominion University by Brian Duvall demonstrated the application of the A-weighting method, as seen in figure 2. The Aeronaut 16x8 3-Blade propeller was tested at a steady operating RPM of 5280 [6]. The unweighted data was compared to the modified A-weighted data. This figure shows how frequencies at the low and high ends of the human hearing range are shifted down as a result of applying the A-weighted curve. 


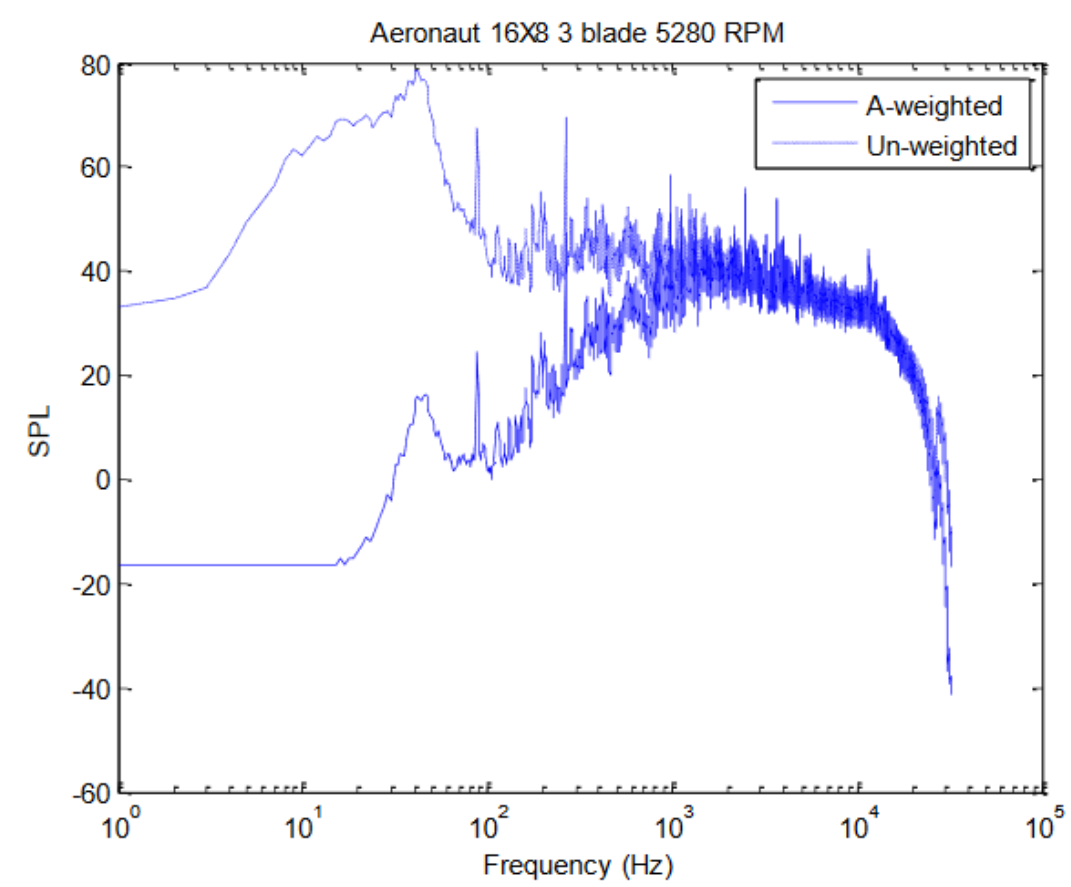

Figure 2: Comparison of Unweighted and A-weighted SPL vs frequency plot [6]

\subsection{PRIOR WORK IN PROPELLER NOISE}

This section reviews older but relevant research involving propeller noise and methods of reducing the acoustic signature. This section goes through, chronologically, the series of discoveries and methods used to investigate propeller noise. It is not a complete history but a brief review of discoveries and insights.

Vogeley [7] conducted tests on reconnaissance planes and found noise could be reduced by lowering RPMs and increasing the number of propeller blades. They achieved a noise reduction of $20 \mathrm{~dB}$ for their reconnaissance planes, but this reduction did not come from the propellers alone. Other factors such as engine mufflers were installed to dampen the noise of the engine exhaust. Regardless, the difference between the on and off state for the propeller was only $5 \mathrm{~dB}$ from a flight altitude of 300 feet. These modifications proved successful in noise reduction for a small light plane flying at low speeds, ranging from 80 to $130 \mathrm{mph}$. 


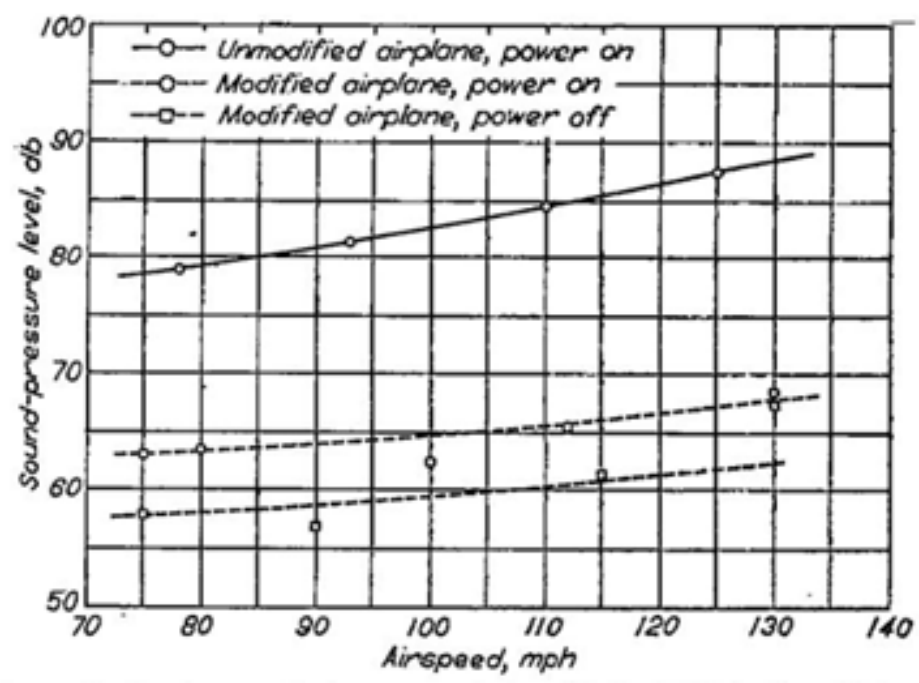

Figure 3: Sound-pressure level measurement recorded from flyover at 300 feet between the unmodified and modified test airplanes. [7]

Harlament [8] developed a 5-blade propeller using a relatively large horsepower engine which operates at low RPM. This propeller, with its high number of blades, low RPM, and low blade loading, resulted in a relatively low-level source of noise. The report also discusses the importance of the Mach number at the tip of the blade and suggests that an elliptical tip would produce less noise than a traditional square-end tip.

Rathgberg and Sipes [9] studied various parameters during aircraft fly-over at $1000 \mathrm{ft}$ altitude. They found tip thickness noise increases with Mach number. In addition, the authors investigated the potential acoustic benefits of shrouding a propeller. They found the shrouds provided insignificant levels of noise reduction.

Lee and Mosher [10] studied several different rotor blade tips for a helicopter. They ran tests at large subsonic tip Mach numbers. They found an appreciable reduction in noise compared to the traditional rectangular rotor blade. However, this noise reduction only exists at subsonic conditions. They found the swept propeller had the least amount of noise at Mach values below 0.9 and the swept-tapered was the second quietest. Simple sweep alone had little effect on high-speed impulsive noise. The blade tip is one of the most important regions for noise production on the blade, with the tip shape having a significant impact on the vortex generated. The thickness noise starts off small but grows to be the dominating source of noise at higher speeds. 

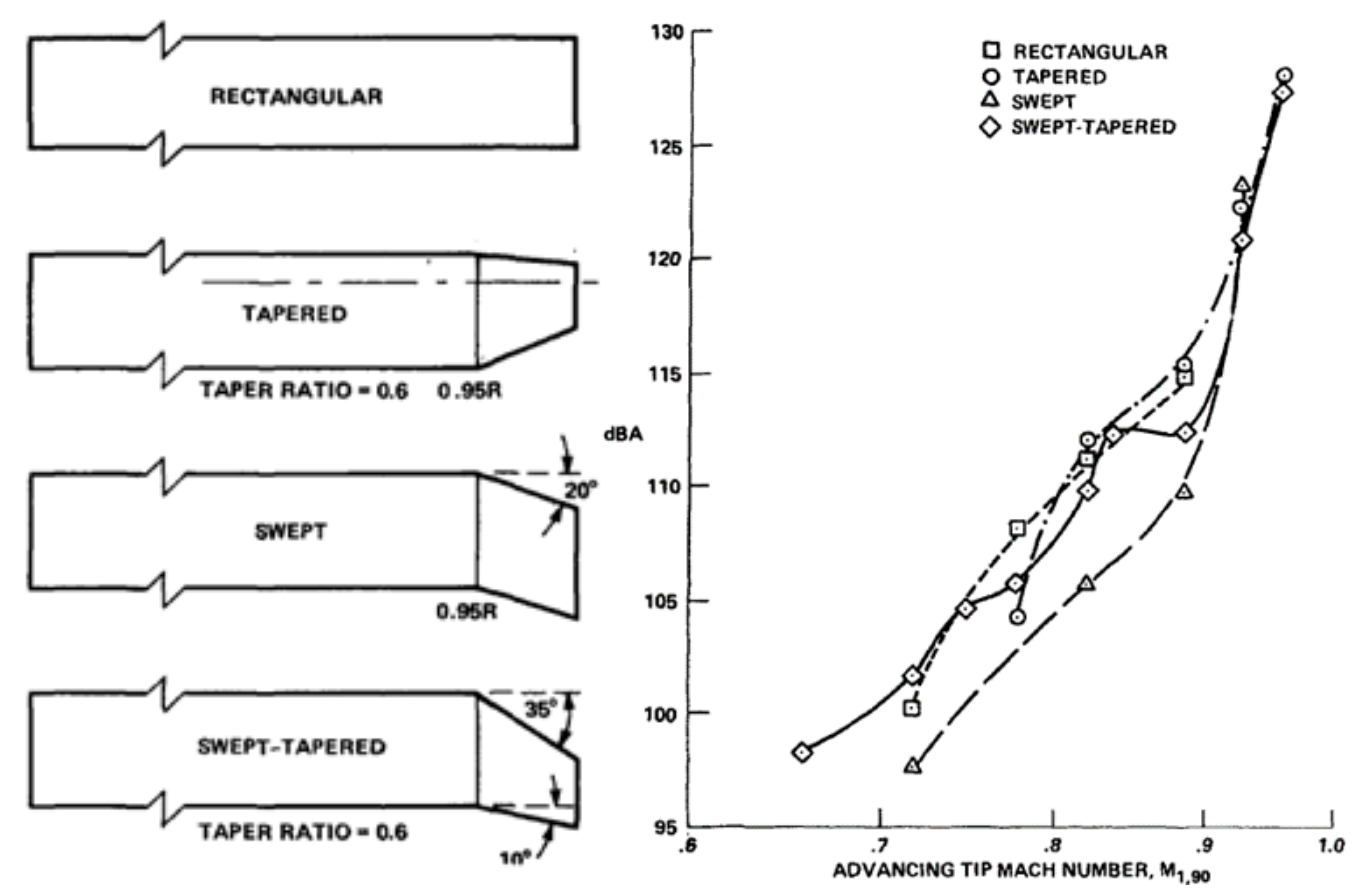

Figure 4a and 4b: 4a shows the four different types of rotor blades tested. $4 \mathrm{~b}$ compares the soundpressure against the tip Mach number for each blade design. [10]

Davis [11] reviewed the impact of different airfoils by comparing the NACA 16 and 65 series airfoils. This test sought to increase blade performance which would allow for a reduction in RPM and ultimately led to a reduction in noise. The study in question compared a 3-blade, 90 inch diameter operating at 2200 RPM to a 3-blade propeller with a redesigned airfoil at 2000 RPM. The test found a $3 \mathrm{~dB}$ reduction while maintaining aerodynamic performance.

Klatte and Metzger [12] studied the single engine Beechcraft Debonair and its various noise sources and found the main source was due to thickness noise. They investigated propeller tip shapes and found that the elliptical tip produced the least amount of noise. Tip sweep was considered in their experiments as well. Tip sweeps as large as 52 degrees were tested and predicted a $5.5 \mathrm{dBA}$ reduction in noise, as shown in figure 5 . The authors also gave several general remarks on their findings:

1- Use elliptical blade tips;

2- Use smaller airfoil tips while maintaining structural support;

3- Optimize propeller performance to reduce diameter;

4- Use as many propeller blades as possible while maintaining performance, cost, and weight criteria. 


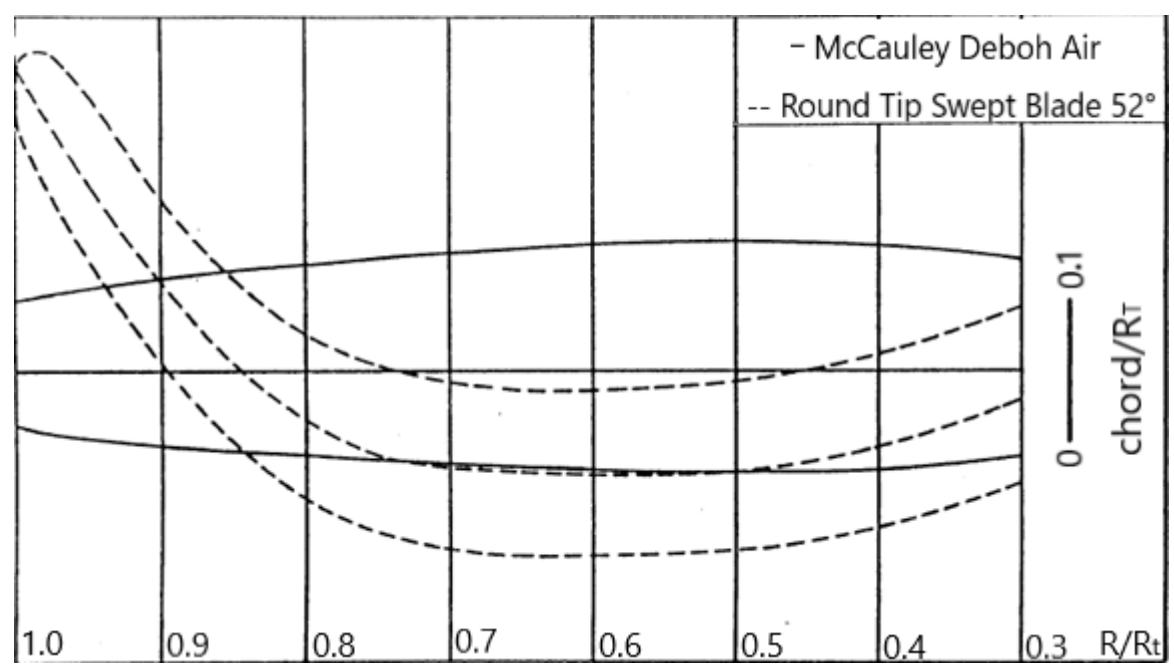

Figure 5: Single Engine Debonair Propeller Blade Planforms [12]

Dobrzynski [13] investigated the use of unsymmetrical blade spacing of a propeller with a large number of blades. An even number of blades must be used to alleviate the concern for balancing. Analytical predictions were conducted on a six-blade propeller, and the optimum angle between blades was found to be 20 degrees apart, producing a noise reduction of $4.3 \mathrm{~dB}$ at Mach values of 0.65 for a 3-meter diameter propeller. It is surprising to note the blade spacing has a stronger reduction for larger diameters, but this trend was only significant for the 6-blade design. The four-blade unsymmetrical spacing did see an initial reduction but no significant trend for larger diameters. A 1.7m diameter propeller was tested inside the DNW wind tunnel, and the general trends agreed with the analytical results. Aerodynamic performance did not suffer as a consequence of unsymmetrical spacing.

Weble [14] reports on a propeller manufacturer's experience in noise reduction. Weble found these general trends:

- $2-3 \mathrm{~dB}$ reduction when the RPM is reduced by 100 ,

- $2 \mathrm{~dB}$ reduction for every $0.1 \mathrm{~m}$ diameter reduction,

- $2 \mathrm{~dB}$ reduction in noise for each additional blade with a reduction in blade diameter.

Chusseau [15] reports on the acoustic and aerodynamic performance of prop-airplane noise. This study used monopole and dipole sources to represent thickness and loading noise respectively. The analysis predicts performance with the curved lifting line theory. Chusseau tested 2-5 bladed propellers at 2700 RPMs. The author found a general trend of $4.5 \mathrm{~dB}$ reduction for each additional blade. However, each 
additional blade interfered with the aerodynamic performance but only minutely as shown in the experiment overview. It should be noted the total radius of the propeller, $\mathrm{R}$, was scaled down for increasing blade count. This was done to keep the same thrust output of $2000 \mathrm{~N}$.

\begin{tabular}{|l|l|l|l|}
\hline Blade count & Scale Size for Propeller & Maximum achievable thrust & Noise reduction \\
\hline $2 \rightarrow 3$ & $R^{*} 100 \% \rightarrow R^{*} 90 \%$ & $0.75 \%$ loss & $-4 \mathrm{~dB}$ \\
\hline $3 \rightarrow 4$ & $R^{*} 90 \% \rightarrow R^{*} 85 \%$ & $1.5 \%$ loss & $-4.5 \mathrm{~dB}$ \\
\hline $4 \rightarrow 5$ & $R^{*} 85 \% \rightarrow R^{*} 80 \%$ & $3.5 \%$ loss & $-4.5 \mathrm{~dB}$ \\
\hline
\end{tabular}

Table 1: A summary of the results composed by Chusseau. [15]

\subsection{MODERN RESEARCH}

This brief historical overview has provided an understanding of the work that has contributed to our modern understanding of rotor acoustics. Research into rotor acoustics dwindled in the 90's and early 2000's. This is likely due to the inherent limitations of conventional propellers and their Mach operation range. Other propulsion designs such as open rotors or "propfans" were investigated due to the capabilities of sustaining flight at higher Mach values. Hager and Vrabel [16] worked on the process of developing advanced blade designs that included sweep in their construction. Geometric shapes of the various propellers are laid out in figure $6 a^{1}$, showing the various tip sweep configurations, number of blades, and operating speeds. The sweep was added to mitigate compressibility while the noise reduction was an incidental benefit. The Single Rotor 3 (SR-3) demonstrated these advantages over the conventional propeller design (SR-2) in figure 6b. The SR-3 obtains both an aerodynamic increase in efficiency and a noise reduction of $5 \mathrm{~dB}$ at Mach 0.8 . It should be noted that the (SR-5) has a dramatic sweep of 60 degrees. The authors pointed out that blade sweep should not exceed this value due to structure concerns.

\footnotetext{
${ }^{1}$ However, it appears that figure $6 \mathrm{~b}$ has a slight typo, as the authors states the SR-2 straight propfan was the loudest and inserted the propeller shape onto the graph in the upper left-hand corner but the SR-1 label has been attached. The SR-1 and SR-2 labels should be switched in figure 6b.
} 

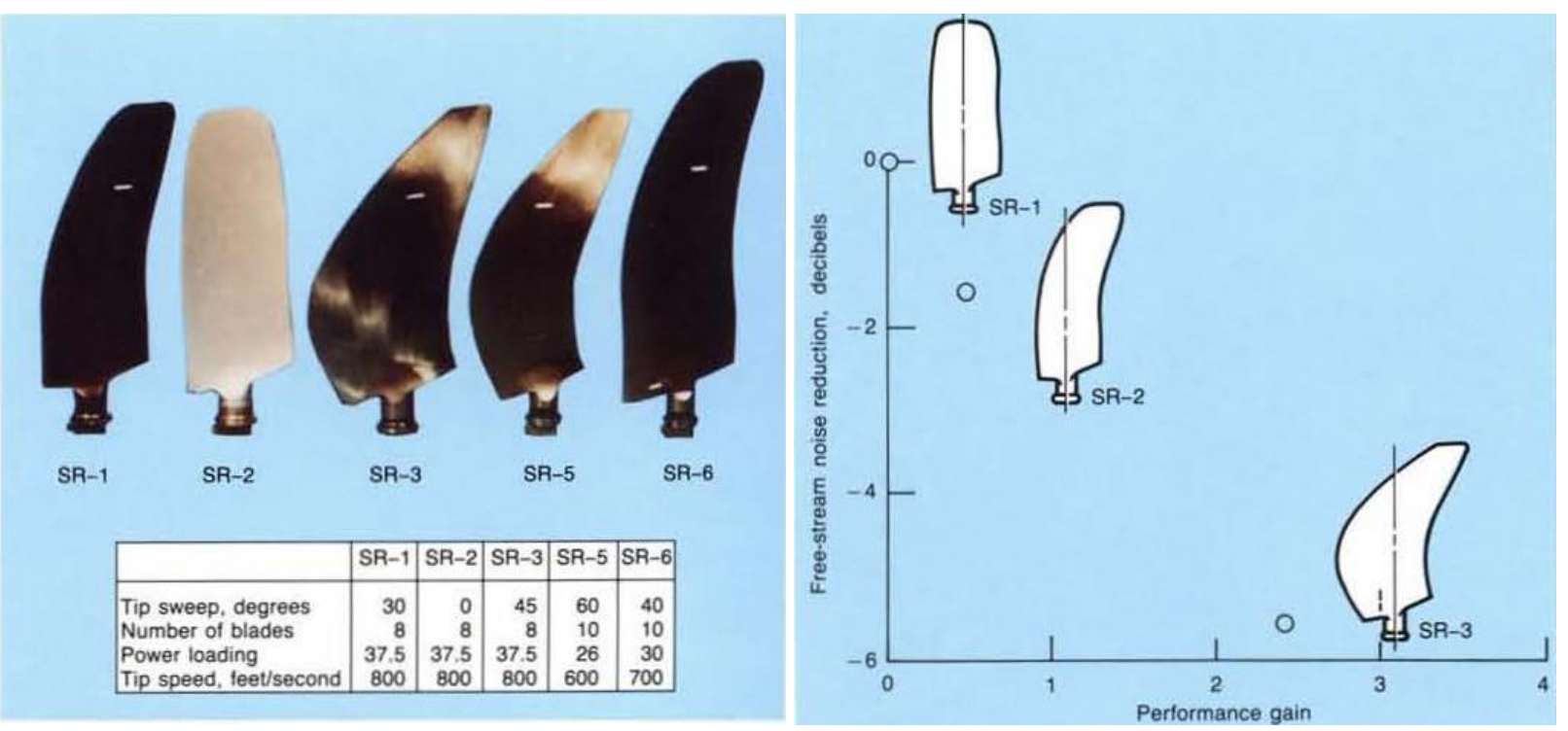

Figure $6 a$ and $6 \mathrm{~b}$ : $6 \mathrm{a}$ shows the various propfan designs from reference 10 . While $6 \mathrm{~b}$ shows the result of applying the swept theory. They reported a $5 \mathrm{~dB}$ reduction in noise and a small increase in efficiency for the swept propeller over the conventional propfan SR-2

With sUAVs in widespread use, propeller research has started to gain interest once again. Kim [17] conducted experimental testing on non-uniform blade spacing, using propellers manufactured with 3D printers. This made the production process quick but leaves the propeller susceptible to loading deformation. The non-uniform spacing of the blades shifts the tonal noise away from the plane of rotation $\left(\theta=90^{\circ}\right)$ with a $5 \mathrm{~dB}$ drop in tonal noise from the plane of rotation but an increase in broadband noise below $\theta=50^{\circ}$. This design could prove beneficial to flyover noise where the propeller's rotational axis is parallel to the ground. Figure 7, from reference 17, shows the non-uniform spacing of the 4-blade propeller and the directions where the benefits of acoustic reduction occur.
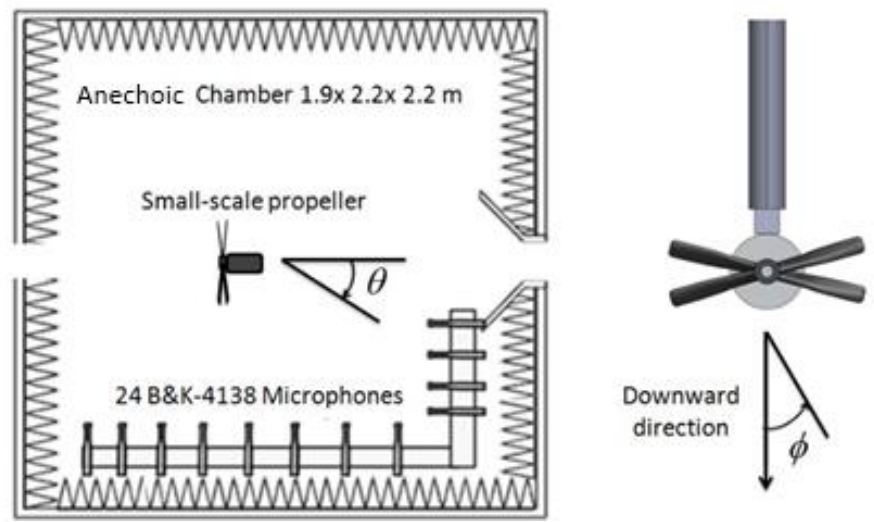

Figure 7: Experimental setup in an Anechoic chamber to evaluate the acoustic non-uniform blade spacing. [17] 
While older studies have focused on the propeller noise characteristics some more recent studies have investigated the electrical motor's and the airframe's contribution to the noise. Huff and Henderson [18] found that strong motor tones can be amplified by propellers loading from anywhere between $5-15 \mathrm{~dB}$. They also concluded that tonal noise is the most significant noise source for propellers. In addition, rotor-airframe interactions (RAI) have shown to be a major contributor to noise, and the closer in proximity the airframe is to the rotor, the more noise that will be generated by the interaction. Zawodny, and Boyd Jr. [19] have compared predictions to experimental testing and found that the RAI can produce greater acoustic pressures than the rotor itself. They have shown that the airframe of the sUAV must be considered for noise reduction. Figure $8 \mathrm{a}$ shows experimental results, and figure $8 \mathrm{~b}$ shows the stark contrast between the rotor and airframe-rotor interaction contribution to the total noise. It should be noted that figures $8 \mathrm{a}$ and $8 \mathrm{~b}$ show the worst case of RAl. At $\Delta / \mathrm{R}=-0.1$, where delta is the clearance space for the rotor tip above the airframe and $\mathrm{R}$ is the radius of the propeller, the RAI dominates the noise contribution, but at $\Delta / R=-0.5$ clearance we see a reduction in noise levels similar to an isolated rotor. This should inform sUAV designers about the construction of their airframes and the importance of clearance. A diagram of the Rotor and Airframe is presented in figure 9.

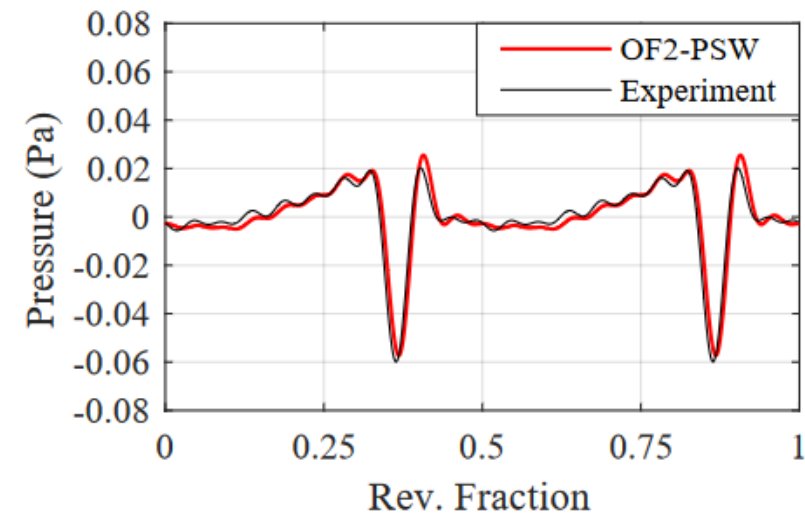

(a) Prediction comparison with experiment

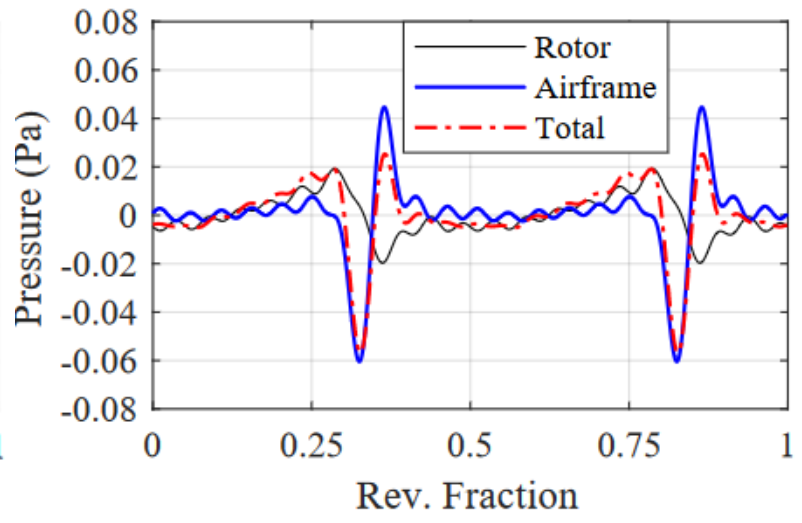

(b) Predicted source contributions

Figure 8: Rotor-airframe noise. Note: Case of $\Delta / R=-0.1, \Omega=5400 R P M,(\theta, \phi)=\left(-45^{\circ},+90\right)$. [19] 


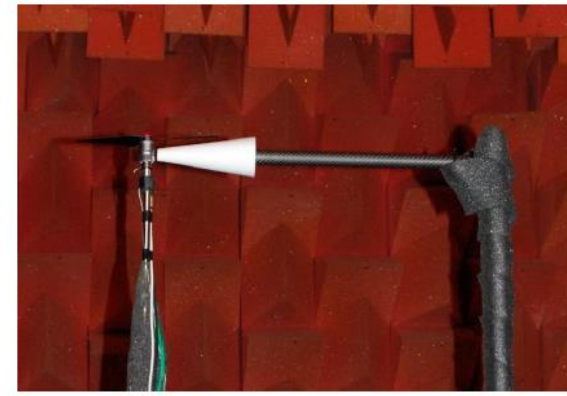

(a) Conical airframe in experimental setup

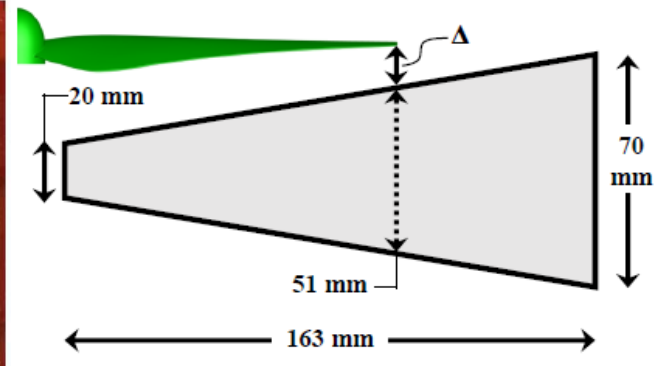

(b) Geometry of conical airframe

Figure 9: Image and geometric details of tested conical airframe [19]

\subsection{SWEPT THEORY}

Previous work established by Hanson [20] demonstrates the theoretical framework for reducing noise with a back swept propeller. Each airfoil along the centerline contributes its own sinusoidal source of

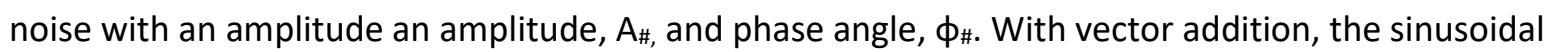
sources can be summed to produce a resultant sinusoidal wave with amplitude, Ar. Figure 10b presents three different propeller blades evaluated with this configuration, where each propeller has a vector addition performed on each respective shape. The conventional blade, on the left side of figure 10b, does not experience any beneficial phase interference from vector addition. Creating a noise source that stacks to form a large resultant amplitude. The next propeller shape has a minor swept shape and experiences some beneficial interference as a result of vector addition. The last propeller has a dramatic sweep, resulting in a strong interference effect. The total noise almost completely closes in on itself, making the resultant amplitude dramatically smaller compared to the conventional design.

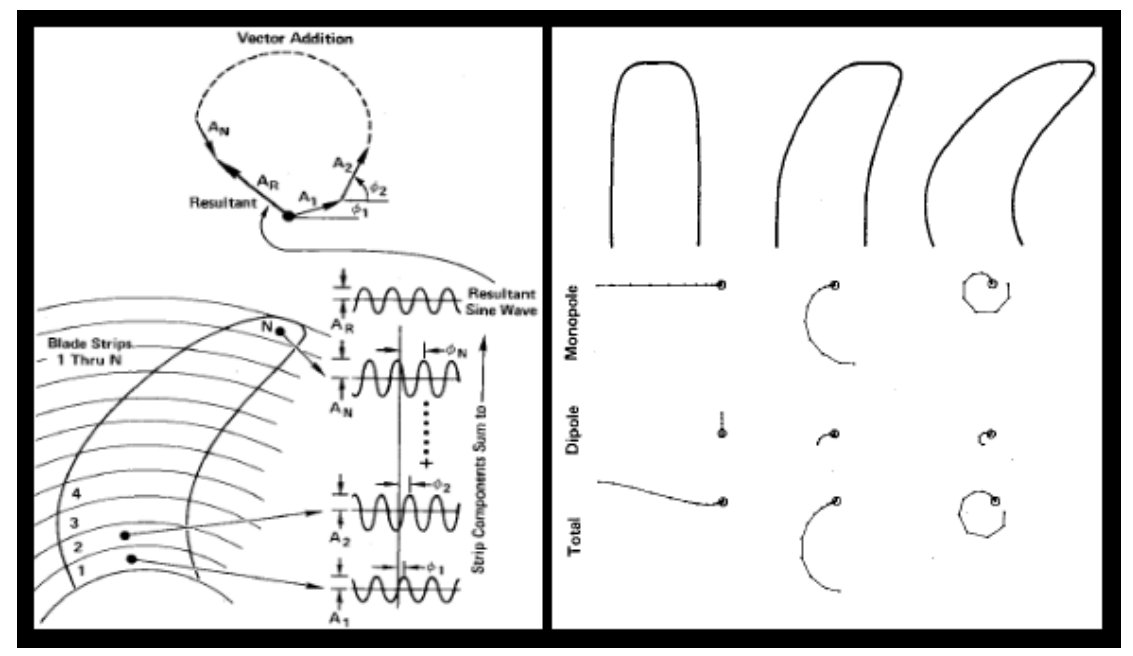

Figure 10a \& 10b: 10a shows the component contribution of each airfoil and $9 \mathrm{~b}$ shows the resultant combination from three different blade configurations. [20] 
While the swept propeller produces a smaller amplitude due to the interference, this effect is highly directional. Figure 11 compares the conventional propeller to the highly swept propeller from the previous diagram. For a propeller positioned in forward flight (plane of rotation at $\theta=90^{\circ}$ ) the effectiveness of the vector cancelation is shown from an observer located at 20,30, and 60-degree angles from forward flight. The upper arch line represents the noise level experienced by observers from the conventional, straight propeller, and the bottom arch displays the noise level produced by the highly swept propeller. The greyed-out region highlights the difference in $\mathrm{dB}$ level between the two geometries. The highly swept propeller has a noticeable benefit between the $10 \mathrm{deg}$ and $50 \mathrm{deg}$ angles with the largest difference in $\mathrm{dB}$ noise between the propeller designs occurring near the 30 deg angle. This angle is optimal because the author prescribed a peak radiation angle at 30 deg in reference 20.

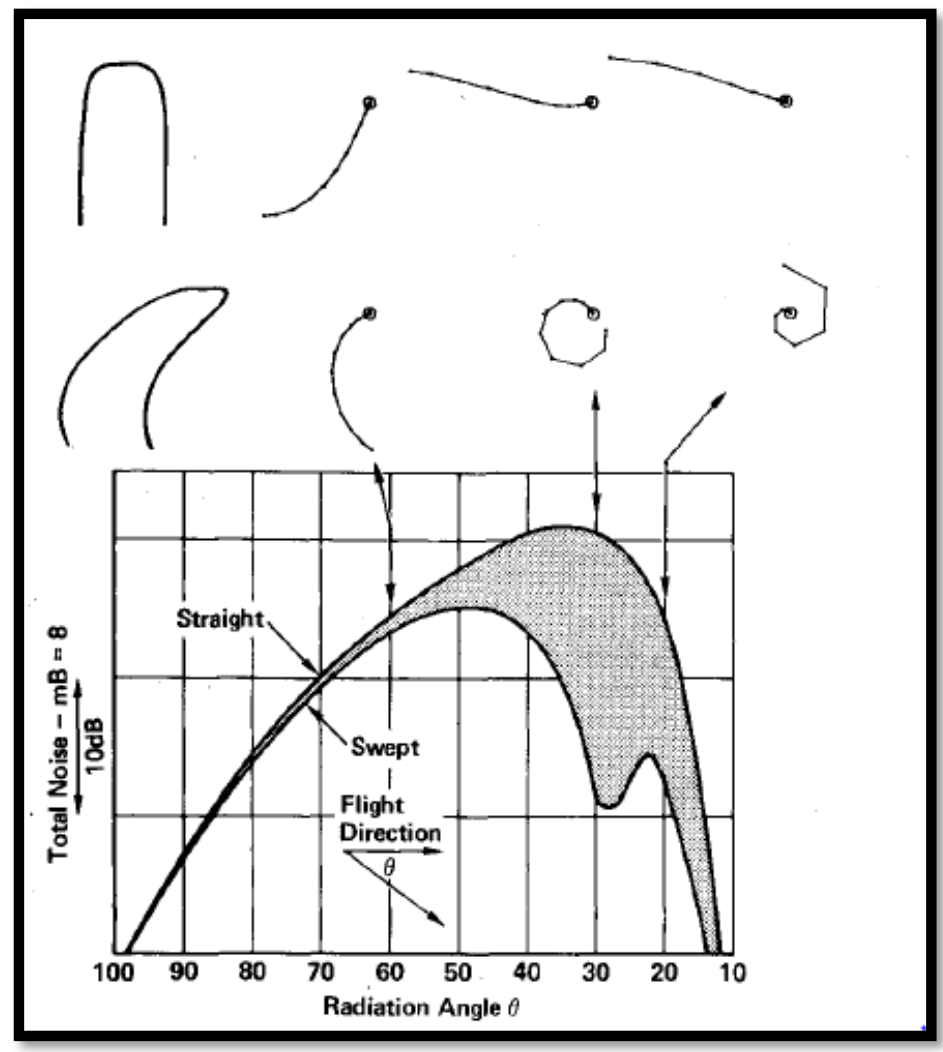

Figure 11: The effectiveness of noise cancelation is highly directional. [20] 


\subsection{BACKGROUND ON PROPELLER AERODYNAMICS}

Since the late nineteenth century, propellers have been used to generate thrust in a fluid environment. Vehicles ranging from submarines to airplanes rely on propellers to generate thrust while suspended in their respective fluid domains. While these propellers operate under different conditions, they still exhibit similar aerodynamic and geometric parameters.

The geometry of a propeller is defined by its tip diameter, D, and its pitch, P. The pitch is the theoretical distance a propeller advances in the axial direction after one revolution. These two dimensions are usually included in a propeller's name, and we will continue to use this practice in our propeller's designs. However, limiting the definition of a propeller blade to tip diameter and pitch is insufficient for describing the 3-D geometry. On a standard propeller there are a series of radial stations, Ra, between the root and the tip where each radial station has an airfoil with a defined chord length, $c_{r}$, and the rotation angle for each airfoil, $\beta$. The series of $R_{a}, c_{r}$, and $\beta$ are often listed in tables where the $R_{a}$ and $c_{r}$ are divided by the radius of the propeller $\mathrm{R}$ as seen in table 2 . Dividing all the chord lengths and radial stations by a user's input allows the geometry to be nondimensionalized. A nondimensionalized geometry allows a user to vary the size of the propeller while keeping the aerodynamic properties the same. This way, a propeller can be made smaller or larger without the need to run a new wind tunnel test. Table 2 shows an example of this relationship with the Master Airscrew 11x8 geometry.

\begin{tabular}{|c|c|c|}
\hline $\mathrm{R}_{\mathrm{a}} / \mathrm{R}$ & $\mathrm{C}_{\mathrm{r}} / \mathrm{R}$ & $\beta$ \\
\hline 0.15 & 0.159 & 35.04 \\
\hline 0.2 & 0.145 & 41.53 \\
\hline 0.25 & 0.146 & 41.45 \\
\hline 0.3 & 0.151 & 37.33 \\
\hline 0.35 & 0.155 & 32.95 \\
\hline 0.4 & 0.158 & 29.67 \\
\hline 0.45 & 0.159 & 26.92 \\
\hline 0.5 & 0.159 & 24.5 \\
\hline 0.55 & 0.158 & 22.46 \\
\hline 0.6 & 0.156 & 20.65 \\
\hline 0.65 & 0.153 & 19 \\
\hline 0.7 & 0.148 & 17.5 \\
\hline 0.75 & 0.143 & 16.29 \\
\hline
\end{tabular}




\begin{tabular}{|c|c|c|}
0.8 & 0.136 & 15.19 \\
\hline 0.85 & 0.128 & 14.03 \\
\hline 0.9 & 0.119 & 12.94 \\
\hline 0.95 & 0.104 & 11.33 \\
\hline 1 & 0.09 & 9.7 \\
\hline
\end{tabular}

Table 2:The geometric properties used to define the 3D shape of a traditional propeller.

The aerodynamics of a propeller can be nondimensionalized. Nondimensionalization of the geometric and aerodynamic properties of the propeller blade allows direct comparison between propellers when they vary in diameter and pitch. The aerodynamic nondimensional performance characteristics can be represented with the following equations. [6]

$$
\begin{gathered}
J=\frac{\text { forward speed }}{\text { radial speed }}=\frac{v}{n * D} \\
C_{T}=\frac{\text { Thrust produced }}{\text { properties of the fluid/propeller }}=\frac{T}{\left(\rho \mathrm{n}^{2} \mathrm{D}^{4}\right)} \\
C_{Q}=\frac{\text { Torque input required }}{\text { properties of the fluid } / \text { propeller }}=\frac{W}{\left(\rho \mathrm{n}^{3} \mathrm{D}^{5}\right)} \\
C_{p}=\frac{\text { Power input required }}{\text { properties of the fluid } / \text { propeller }}=\frac{2 \pi n W}{\left(\rho \mathrm{n}^{3} \mathrm{D}^{5}\right)} \\
\eta=\frac{\text { propulsive power out }}{\text { shaft power in }}=J \frac{c_{T}}{C_{P}} \\
\eta_{(J=0)}=\text { static efficiency }=\frac{T^{\frac{3}{2}}}{2 \pi n W * \sqrt{2 \rho A}}
\end{gathered}
$$

Performance plots for the MasterAirscrew $11 \times 8$ propeller are shown in figures $12 a, 12 b$, and 13 . These figures illustrate the general trends for coefficient of thrust, power, and efficiency for a propeller operating under steady flight as the advance ratio, J, increases. As noted in equation (2), J represents the nondimensional airspeed which compares the airstream velocity with respect to the cyclorotor tip speed $(n * D)$. $J$ is the parameter along the horizontal axis for all the performance plots. This metric is gradually increased for the three aerodynamic properties of interest. Figures $12 \mathrm{a}$ and $12 \mathrm{~b}$ show a decrease in $C_{t}$ and $C_{p}$ as $J$ increases for a constant tip speed. 

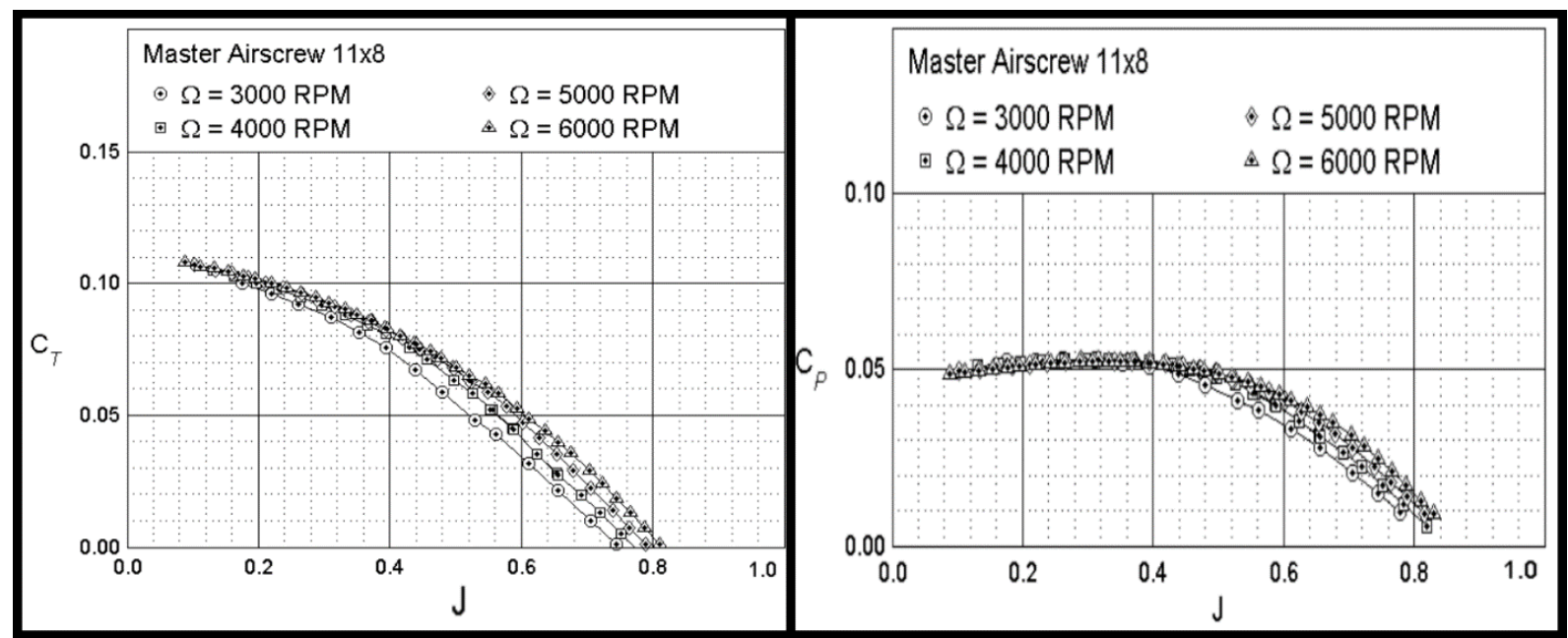

Figure 12a and 12b: MasterAirscrew 11x8 Performance Characteristics.[21]

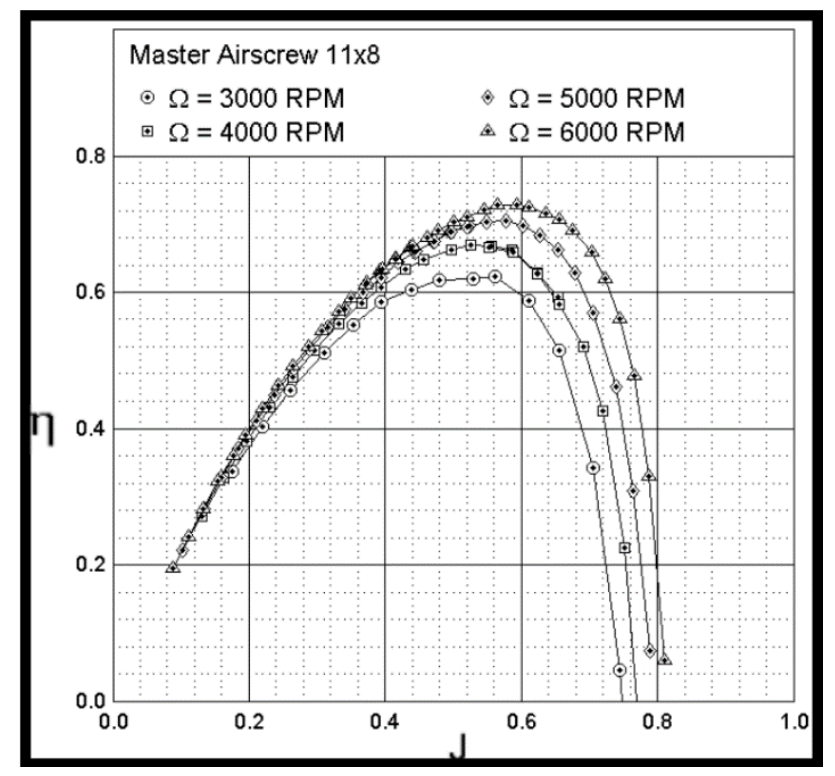

Figure 13: Master Airscrew 11x8 efficiency trends [21]

To understand why thrust and torque decrease as advance ratio increases, one should look at the blade element theory. The velocity vector for a propeller can be broken into two components, a radial component based on the rotational speed and the airstream velocity. The vector component creates an angle with the resultant velocity, phi. This can be seen in figure 14. Note that the beta angle is the total angle for the airfoil. The difference between the angle beta and phi is alpha, which represents angle of attack. As the advance ratio, or non-dimensional speed, increases the airspeed velocity component increases resulting in a larger phi angle. Since Beta is a geometric constant, only alpha can decrease to 
account for the larger phi angle. With this loss in angle of attack we get a loss in lift and by extension a loss in thrust. The thrust is reduced to zero when $\phi=\beta$. This is marked as the Zero Lift Line in figure 14 .

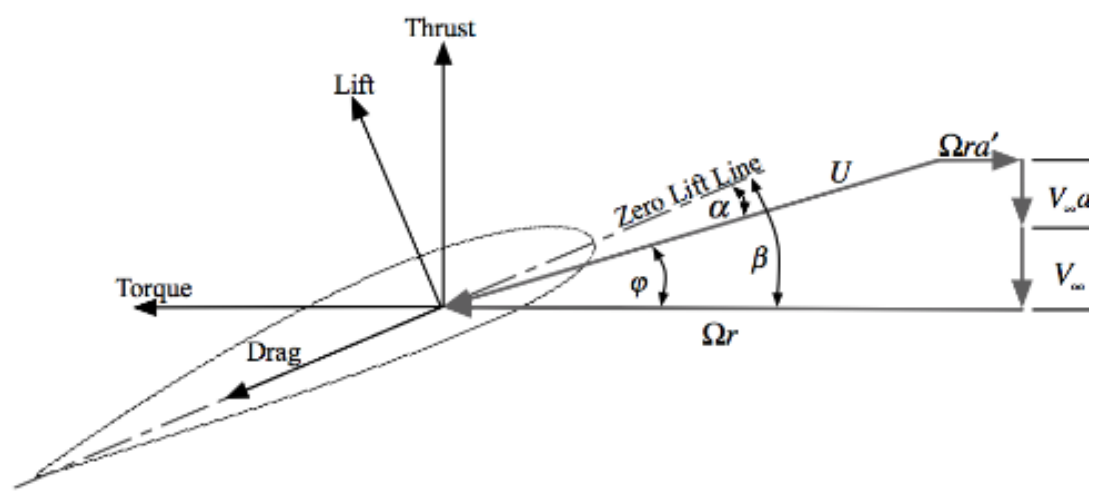

Figure 14: Blade element aerodynamic forces [22]

The $C_{t}$ and $C_{p}$ trends are compared with our "measurement of goodness," efficiency, from equation (6) which is represented with the Greek letter $\eta$, where $\eta$ evaluates the ratio between the useful power produced against the input power required to operate. It should be noted that the optimum condition, where the best tradeoff for thrust versus power input exists, occurs at $\mathrm{J} \sim 0.6$ for the Master Airscrew 11x8 propeller according to figure 13. The wind tunnel data was taken from the UIUC Propeller Database [21]. 


\section{CHAPTER 3}

\section{ACOUSTIC THEORY}

\subsection{ACOUSTIC SOURCES}

Equation (8) shows the one-dimensional wave propagation differential equation and equation (7) provide the three-dimensional wave propagation equation. This equation is useful in describing the motion of a vibrating string or sound radiating through a pipe.

$$
\begin{aligned}
& \frac{1}{c^{2}} \frac{d^{2} p^{\prime}}{d t^{2}}-\frac{d^{2} p^{\prime}}{d x^{2}}=0 \\
& \frac{1}{c^{2}} \frac{d^{2} p^{\prime}}{d t^{2}}-\nabla^{2} p^{\prime}=0
\end{aligned}
$$

\subsection{MONOPOLE}

This particular derivation of the Monopole and Dipole is taken from reference [23]. The classical wave equation, eqs. (8) and (9), are sufficient for describing the behavior of propagation if the forces and motion fluctuations in the medium are kept small. If there is excitation in the acoustic fluctuations caused by turbulence in the flow or a geometry rapidly moving through the medium, then there is an imbalance in the field. This can be accounted for with a source function $q(y, t)$, where $y$ represents the position, in vector coordinates, of the source and $t$ represents time. The modified nonhomogeneous wave equation with one source function is.

$$
\frac{1}{c^{2}} \frac{d^{2} p^{\prime}}{d t^{2}}-\nabla^{2} p^{\prime}=q
$$

The nonhomogeneous PDE can be solved with Green's function for the wave equation in unbounded space to produce equation (11). Note, $x$ and $y$ are vectors.

$$
p^{\prime}(x, t)=\int_{V} \frac{q\left(y, t-\frac{|x-y|}{c}\right)}{4 \pi|x-y|} d^{3} y=\int_{V} \frac{q\left(y, t-\frac{r}{c}\right)}{4 \pi r} d^{3} y
$$

The 4pi $|\mathrm{x}-\mathrm{y}|$ is a product of Green's function. This expression accounts for the spherical spreading of the source function and represents the distance between the observer position vector, $x$, and the source position vector, $y$. The distance between the two is often represented with $r$ instead of $|x-y|$. The $t-\mid x-$ $\mathrm{yl} / \mathrm{c}$ expression accounts for the arrival time delayed by the speed of propagation from the source to the observer. This expression is integrated over the spatial region the source volume occupies. If we represent the source function as a point source, we can remove the source position vector and purely represent the source as a function of time. This gives the relationship below. 


$$
p^{\prime}(x, t)=\frac{Q\left(t-\frac{r}{c}\right)}{4 \pi r}
$$

For the monopole case, which is represented as a single frequency, sinusoidal wave, the source strength can be found with equation (13), where Phi is the phase shift, $Q_{0}$ is the mean offset, and $Q_{s}$ is the complex source amplitude to account for losses. Ultimately, we are only concerned with the real part of the source.

$$
p^{\prime}(r, t)=\operatorname{Real}\left\{\frac{Q^{*}{ }_{s} e^{i(\omega t+\varphi)}+Q_{0}}{4 \pi r}\right\}
$$

Reference 23 also provides the derivation to include the Doppler effect and acoustic velocity. In the present work, there is little concern with that portion of the paper as the simulations is not concerned with these effects. Figure 15 shows a top down view of a monopole propagating acoustic pressure in all radial directions at a constant frequency.

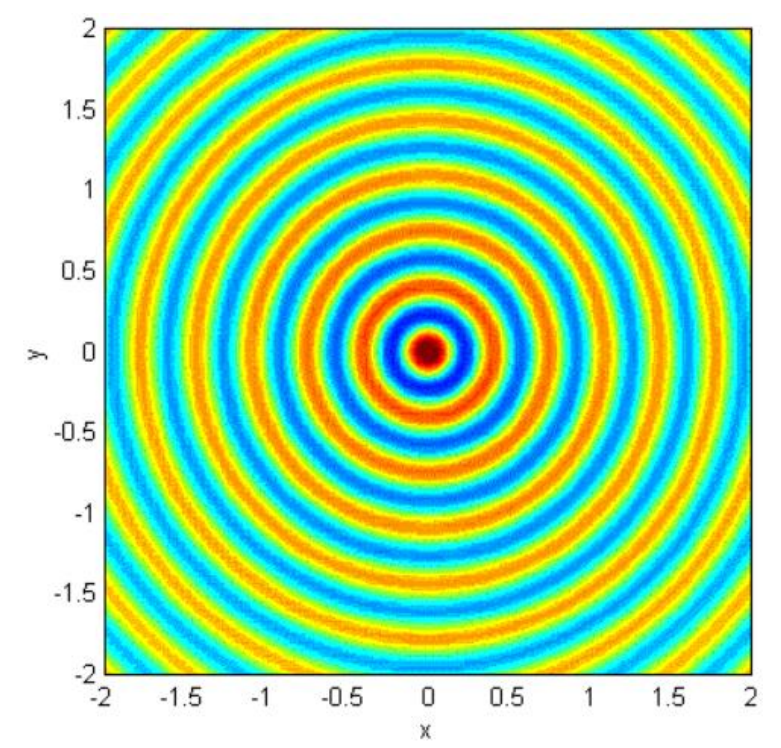

Figure 15: Top down view of the monopole propagation.

\subsection{DIPOLE}

A dipole is a source composed of two monopoles in close proximity propagating at 180 degrees out of phase with each other. This means that when one pole has a strength of $+Q$ the second pole's strength will be $-Q$. It should be noted the distance, $d$, between the poles is small but it is still greater than the diameter of either point source. As these sources approach each other, the combined source strength equation takes the following form: 


$$
Q(t)\{\delta(x)-\delta(x-d)\}=-\nabla *\{Q(t) d * \delta(x)\}
$$

The source strength for a dipole is slightly different than the monopole since the single sinusoidal waves. This source strength has a magnitude of $Q(t)$ and is highly directional. This source strength will be represented with $F(t)$ and results in a dipole pressure response found in equation (15), which shares similarities with the monopole pressure field as a point source in equation (9).

$$
p^{\prime}(x, t)=\frac{F\left(t-\frac{r}{c}\right)}{4 \pi r}
$$

The source strength, $F(t)$, has a harmonic time dependence with an offset, similar to the $Q$ term found in equation (14). The pressure solution to the dipole expression is in terms of $r, \theta$, and $t$. This distinction is important as the out-of-phase monopoles create interference in the far field preventing uniform propagation.

$$
p^{\prime}(r, \theta, t)=\operatorname{Real}\left\{\frac{\cos (\theta)}{4 \pi r} *\left[F_{S}^{*}\left(\frac{1}{r^{2}}+\frac{i \omega}{r c}\right) e^{i(\omega t+\phi)}+\frac{F_{0}}{r^{2}}\right]\right\}
$$

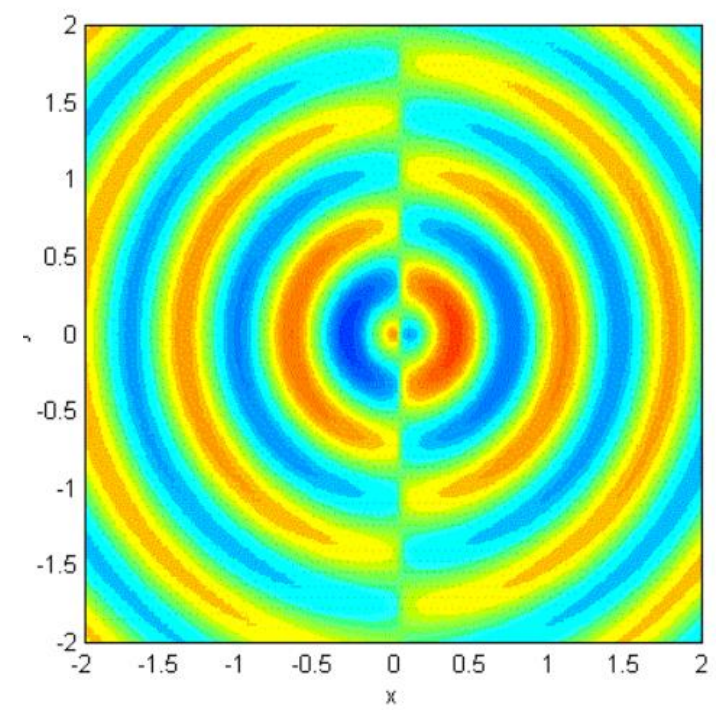

Figure 16: Top down view of a dipole propagation.

\subsection{THICKNESS NOISE}

When a physical body moves through a medium a pressure disturbance is created as a result of the fluid displacement. Larger and faster moving objects will create stronger wakes in the medium. This displacement is present in rotor dynamics where a propellers airfoil displaces fluid. The effect can be modeled as a monopole source and is known as thickness noise. Thickness noise is predominantly directed in the plane of the rotor. [24] 


\subsection{LOADING NOISE}

Loading noise is a result of steady and unsteady forces acting on the medium. For a propeller, these forces are broad band, lift, and drag. Loading noise predominantly act in the aft direction of the propeller [24] and can be represented as a dipole source. While it is helpful to approach complex noise sources in terms of their fundamental source functions, it is misleading to state that the monopoles sources are equivalent to thickness noise and dipole sources are equivalent to loading noise because a moving source signature is different from a stationary source [25]. For this reason, future discussion will refrain from discussing rotor noise in terms of monopoles and dipoles.

\subsection{FFOWCS WILLIAMS \& HAWKINGS EQUATION}

The Ffowcs Williams and Hawkings (FW-H) equation (17) governs the generation of sound in fluids from the presence of solid bodies in motion. The FW-H is an nonhomogeneous wave equation that manipulates the continuity equation with the Navier-Stokes equation into one volume and two surface sources for noise generation, where each source term can be thought of as distinct physical phenomenon contributing to the total noise produced by the moving body [26]. This makes the FW-H equation insightful for investigating noise generation as the components of the total noise can be separated into their individual physical contributions and studied. The FW-H equation involves enclosing all sound sources within a control surface, $f(x, t)=0$. This mathematical representation of the control surface allows us to represent $\nabla f=n$, where $n$ is the unit outward normal to the surface. The control surface can either represent an impermeable surface, such as a propeller, or a permeable surface surrounding the propeller.

$$
\overline{\square^{2}}=\frac{1}{c^{2}} \frac{d^{2}}{d t^{2}}-\nabla^{2}=\frac{d^{2}}{d x_{i} d x_{j}}\left\{T_{i j} H(f)\right\}+\frac{d}{d t}\left\{U_{n} \delta(f)\right\}-\frac{d}{d x_{i}}\left\{L_{i} \delta(f)\right\}
$$

\begin{tabular}{|c|c|c|}
\hline Component & Permeable surface & Impermeable surface \\
\hline$U_{n}$ & $=\left(\left(\rho_{0}-\rho\right) v_{n}+\rho_{0} u_{n}\right) * \widehat{n}_{J}$ & $\rho_{0} v_{n} \widehat{n}_{J}$ \\
\hline$L_{i}$ & $=\left(p_{i j}+\rho u_{i}\left(u_{n}-v_{n}\right)\right) * \widehat{n_{J}}$ & $p_{i j} * \widehat{n}_{J}$ \\
\hline$T_{i j}$ & $\rho u_{i} u_{j}+\left[c_{0}{ }^{2}\left(\rho-\rho_{0}\right)\right] \delta_{i j}+p_{i j}$ & $\rho u_{i} u_{j}+\left[c_{0}{ }^{2}\left(\rho-\rho_{0}\right)\right] \delta_{i j}+p_{i j}$ \\
\hline
\end{tabular}

Table 3: Permeable and Impermeable source terms 
The first component in equation (17) is the volume source term which can be identified by the Heaviside function $\mathrm{H}(\mathrm{f})$ and the $\mathrm{T}_{\mathrm{ij}}$ term, which represents the Lighthill stress tensor. This stress tensor contains all the nonlinear contributions to the rotor noise. The next two sources are Thickness noise (red) and Loading noise (blue) and can be identified by the delta function, $\delta(f)$, attached to their respective source terms. Table 3 shows two forms of $U_{n}$ and $L_{i}$ that can be used to represent the variables. The placement of the control surface, $f$, determines which source term definition is used. If the surface is impermeable, then the control surface is the propeller blade. If the control surface exists some distance away from the propeller, then the permeable terms are used. The permeable terms are often used in CFD solutions to calculate the near-field acoustic generation and propagation. The CFD/FW-H approach has been applied to many complex geometries such as helicopters, open rotors, slats, flaps, trailing edges, jets, and wind turbines [27]. Figure 17 provides a description of a surface in motion. This figure shows the locations of the interior boundary, $f(x, t)<0$, the exterior boundary, $f(x, t)>$ 0 , and the control surfaces, $f(x, t)=0$.

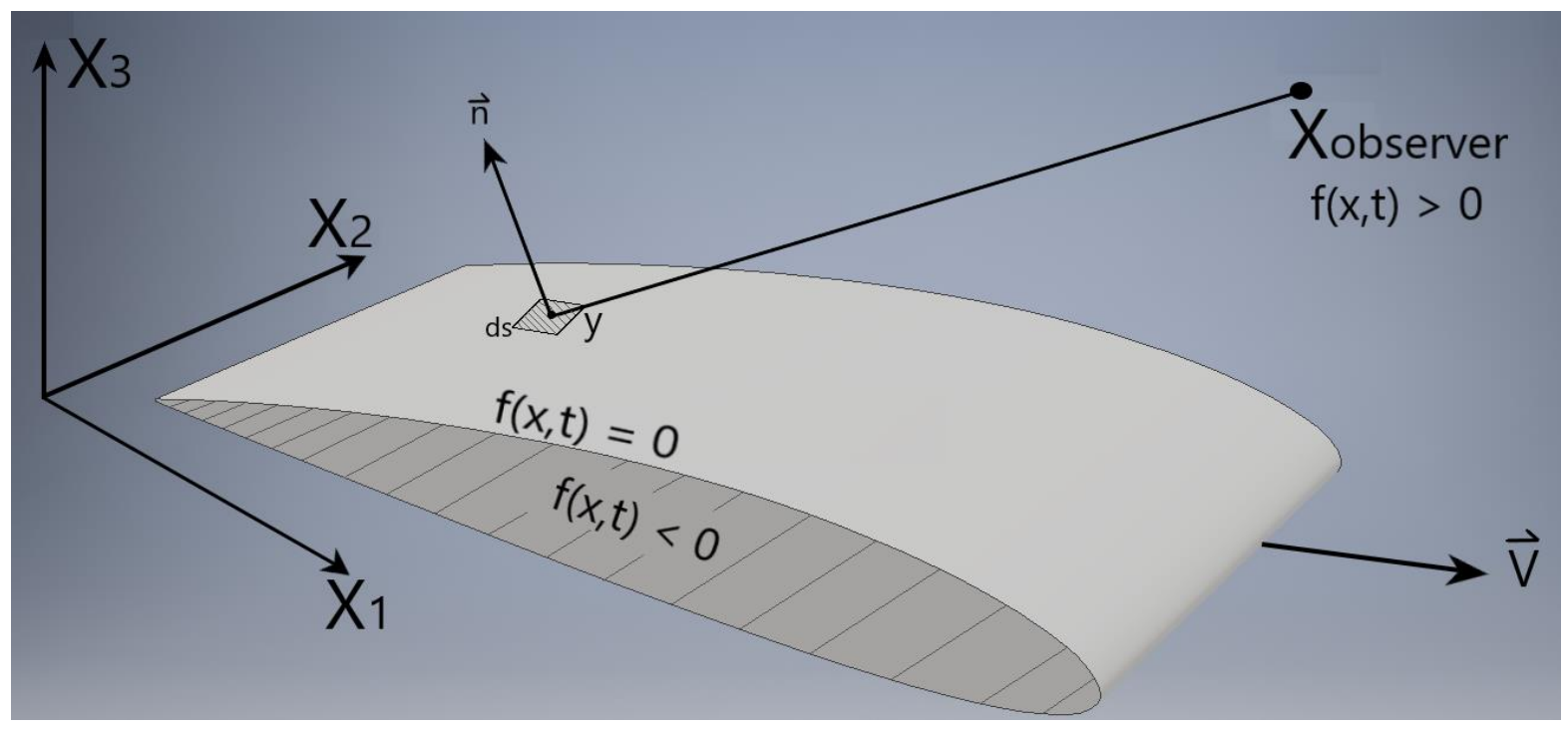

Figure 17: The definition of the moving surface as $f(x, t)=0$. This sets $n$ as the unit outward normal to the surface. $F(x, t)>0$ and $f(x, t)<0$ represents the interior and exterior respectively.

This has made $\mathrm{FW}-\mathrm{H}$ and the related formulation (Farassat formulation 1 and $1 \mathrm{~A}$ ) useful for studying rotor and propeller acoustics, assuming the medium is at rest. The $\mathrm{FW}-\mathrm{H}$ equation used in this study relies on the Farassat formulation $1 \mathrm{~A}$ which converts the differential terms from the original $\mathrm{FW}-\mathrm{H}$ equation to an integral form. This formulation provides a relatively low computational cost and a general robustness [28]. The results of the derivation with Farassat $1 \mathrm{~A}(\mathrm{~F} 1 \mathrm{~A})$ are seen in equation (19) 
for the thickness noise and equation (20) for the loading noise. The exact derivation can be found in reference [26]. Farassat methodically takes the reader from the $\mathrm{FW}-\mathrm{H}$ differential form to the F1A Integral formulation with a detailed explanation of the derivation. In addition, he provides background material relevant to the derivations. It should be stated that the F1A derivation assumes an impermeable source and the contributions from the volume source have been neglected. This leaves us with equation (18) where we are only concerned with the thickness and loading noise.

$$
\begin{gathered}
p^{\prime}(x, t)=p^{\prime}{ }_{T}(x, t)+p^{\prime}{ }_{L}(x, t) \\
4 \pi p^{\prime}{ }_{T}(x, t)=\int_{f=0}\left[\frac{\left(\dot{U}_{n}+U_{\dot{n}}\right)}{r\left(1-M_{r}\right)^{2}}\right]_{r e t} d S+\int_{f=0}\left[\frac{\dot{U}_{n}\left(r \dot{M}_{r}+c M_{r}-c M^{2}\right)}{r^{2}\left(1-M_{r}\right)^{3}}\right]_{r e t} d S \\
4 \pi p^{\prime}{ }_{L}(x, t)=\frac{1}{c} \int_{f=0}\left[\frac{\dot{L_{r}}}{r\left(1-M_{r}\right)^{2}}\right]_{r e t} d S+\int_{f=0}\left[\frac{L_{r}-L_{M}}{r^{2}\left(1-M_{r}\right)^{2}}\right]_{r e t} d S+\frac{1}{c} \int_{f=0}\left[\frac{L_{r}\left(r \dot{M}_{r}+c M_{r}-c M^{2}\right)}{r^{2}\left(1-M_{r}\right)^{3}}\right]_{r e t} d S
\end{gathered}
$$

As noted in the reference, variables with a subscript imply a dot product operator with the unit outward normal vector $(n)$, radiant vector $(r)$, or surface velocity vector normalized by the speed of sound (M) and any variable with a dot accent requires a source time derivation. The $1 / \mathrm{r}^{2}$ identifies the near-field terms and the $1 / r$ shows the terms that are relevant in the far-field. All the integral terms are bracketed with the subscript ret. This is the retarded time and implies the integration is evaluated over the duration of time that the sound is measured by the observer. Equation 21 provides the mathematical relation for retarded time. Retarded time, $\tau$, is the time it takes the information to reach the observer, $t$, due to the distance between the source and the observer, $r$, over the finite speed of the medium, $c$.

$$
\tau=t-\frac{r}{c}=t-\frac{|x-y|}{c}
$$




\section{CHAPTER 4}

\section{COMPUTATIONAL APPROACHES}

\subsection{XROTOR}

XROTOR is an interactive program designed for analyzing ducted and free-tip propellers. The program was developed by Mark Drela and Harold Youngren of MIT [29]. XROTOR allows a user to input an arbitrary rotor geometry and run analyses on the aerodynamic characteristics at a given RPM. In addition, XROTOR can optimize on various geometric properties such as twist distribution and perform structure and acoustic analysis on the propeller, but the acoustic features XROTOR provides are limited for the swept propeller investigation as XROTOR assumes a conventional blade design. XROTOR is used to calculate the aerodynamic characteristics of the unswept propeller at various RPMs. Each RPM evaluation result in a pressure distribution around the blade, giving us a coefficient of lift and drag for each airfoil station. After evaluation, XROTOR's coefficient of thrust and torque will be compared against the wind tunnel data collected in ODUs Closed-Circuit Low-Speed Wind Tunnel. The comparison serves as a check on the pressure distribution which are incorporated into a second computational program to calculate the acoustic noise, ANOPP2.

\subsection{AIRCRAFT NOISE PREDICTION PROGRAM 2 (ANOPP2)}

ANOPP2 is a toolkit that is designed to predict, with high accuracy, noise produced by an aircraft, regardless of whether the aircraft under study is a conventional design, that has previous data to create semiempirical models, or an unconventional design, without previous data[30]. ANOPP2 uses physics-based features to analyze an aircraft and gain insight into the noise generating mechanisms. These physics-based features included noise produced by the propulsion devices and their interaction with the airframe while accounting for real-world flight effects such as flight path (for the doppler effect) and atmospheric/terrain effects. With these physic-based features, which improves upon its predecessor ANOPP, empirical/semi-empirical methods are no longer necessary to predict noise. An overview of the capabilities of ANOPP2 are briefly discussed and displayed in figure 18. The following excerpt points are taken from reference 30, describing the objectives of ANOPP2.

\section{Objectives for ANOPP2}

- Provide the capability to predict the noise from arbitrary aircraft designs, including conventional and unconventional aircraft designs of full-scale and model-scale size, as well as Unmanned Aerial Vehicles (UAVs), Personal Aerial Vehicles (PAVs), and other external acoustic applications. 
- Establish a framework where a combination of acoustic prediction methods of varying fidelity can communicate in a unified system. This ranges from fast computations with reduced order models for system level assessment and design to potentially computationally intensive, high-fidelity, physics-based methods for investigating and understanding noise generation and propagation effects at the components and aircraft system level.

- Include acoustic effects for comparison to flight and model-scale test data such as flight test environment, Doppler effects, convective amplification, and propagation.

- Include physics-based prediction of Propulsion Airframe Aeroacoustics (PAA10), airframe interaction effects such as scattering of landing gear noise by the airframe, and propulsion interactions like those seen in closely spaced twin jets or open-rotors.

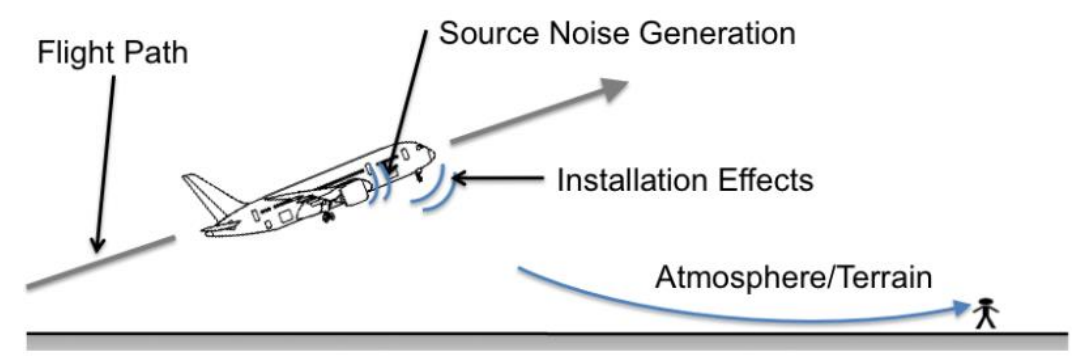

Figure 18: ANOPP2 incorporates various physical effects into the physics-based analysis. [30]
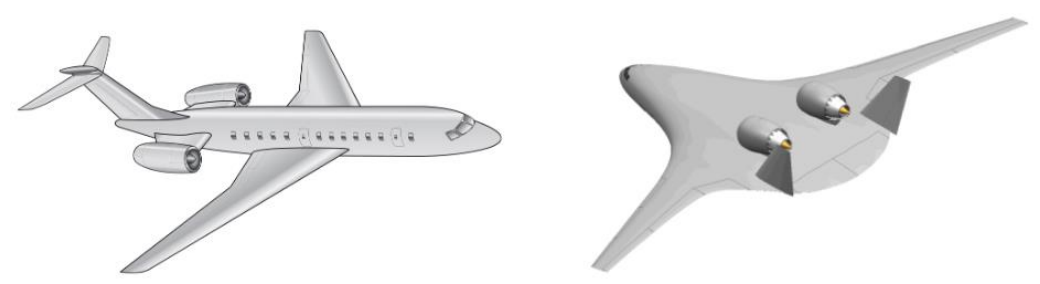

Figure 19: Conventional tube-and-wing and unconventional Hybrid Wing Body aircraft designs. [30]

As shown in reference 30, ANOPP2 is useful in predicting noise for unconventional aircraft designs, which suits the interests of the swept propeller blade investigation. In addition, ANOPP2 can have multiple noise sources evaluated while operating under the physics-based features. This makes it possible to test the swept theory with a series of noise sources placed incrementally from root to tip on each prototype propeller. Figure 18 presents a comparison between a conventional and nonconventional aircraft which ANOPP2 can model and predict. 


\section{CHAPTER 5}

\section{DEFINITION OF SWEPT PROPELLER AND MANUFACTURING 5.1 DEFINITION OF DESIGN FAMILY}

As discussed in the Background on Propeller Aerodynamics section, there are several physical characteristics required to define the geometry of a conventional propeller. The swept propeller builds on these characteristics with two additional parameters. The first parameter is the sweep-back angle. This angle defines where the tip of the propeller is placed with respect to the conventional centerline. In figure 20, a swept propeller is given a swept-back angle of 35 degrees. This means the airfoil at $R_{a} / R=$ 1.00 is rotated 35 degrees back from the original centerline. Each black circle represents a radial distance, $R_{a} / R$, in space and the blue line represents the centerline path of the propeller blade. The solid line representing the modified position and the dotted line representing the original position.

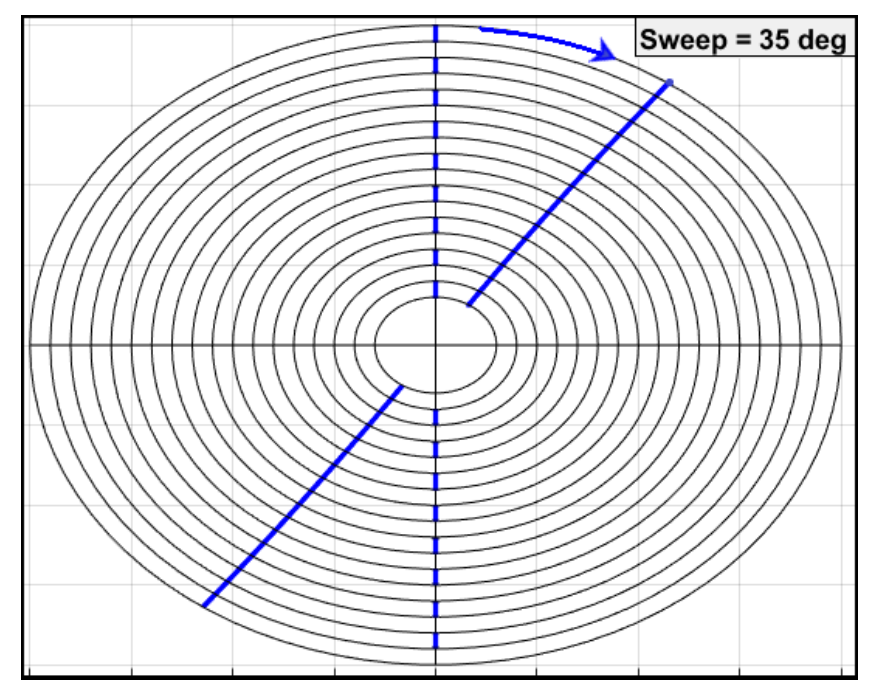

Figure 20: First parameter, sweep-back angle, applied to a propeller.

The sweep-back angle has shifted the centerline path but the second parameter, radius of curvature (Rc), will define the magnitude of curvature for the swept blade. This parameter gives the propeller its "bowing" shape. Figure 21 provides a visual demonstration of applying the Rc to create a bowed centerline. Figure 21a has the swept-back centerline from the previous figure with two new circles, of radius $\mathrm{Rc}$, centered at the origin and the tip. The overlapping point between these two circles identifies the position of the radius of centered curvature (RCC). The RCC is a vital component for constructing the curve and will be marked with a red star. The next step is carried out in figure $21 \mathrm{~b}$, where an additional circle, marked in red, is drawn with a radius of Rc and centered at the RCC. This circle defines the 
geometric sweep of the propeller. The conventional centerline path is now bowed with respect to the RCC and follows the red circle. Figure 21c shows the shape of the new centerline path and figure 21d gives a top-down view of the new centerline path and the isosceles triangle that was created as a result of defining the overlapping point. The isosceles triangle will be used later to define the location of the individual radial stations.

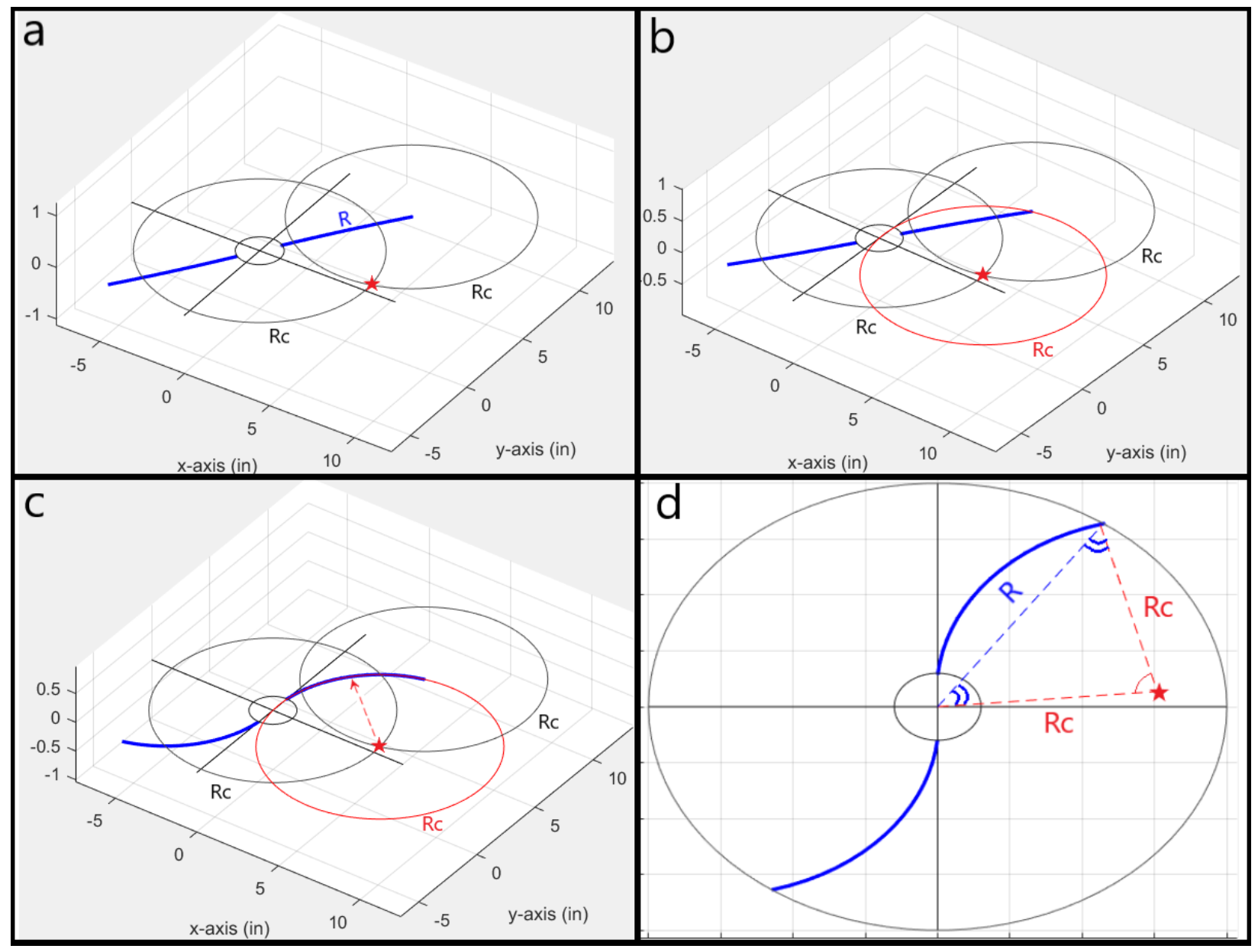

Figure 21: The process of constructing the geometric sweep with the two new inputs.

The geometric sweep has been defined for this propeller family, but the geometry should remain nondimensionalized. This can be easily done by equating $R_{c}$ to $R$ and multiplying by some constant. For this example, when a propeller is prescribed a radius of 8 inches, $R_{c}$ is defined as

$$
\begin{gathered}
\mathrm{R}_{\mathrm{c}}=0.775^{*} \mathrm{R} \\
\mathrm{R}_{\mathrm{c}}=6.2 \mathrm{in}
\end{gathered}
$$


The constant 0.775 was chosen after iterating on various $R_{c}$ values until the first airfoil, $R_{a} / R=0.15$, was set to $0^{\circ}$. With the first airfoil station fixed to the conventional centerline and the last airfoil fixed $35^{\circ}$ off the conventional centerline, the arc path must perform a sweep of 35 degrees from root to tip. With the swept shape defined, the next step is to define each airfoil's radial position, $R_{a} / R$, on the new centerline. Figure $21 \mathrm{~d}$ shows the isosceles triangle that was created as a result of defining the sweep, and figure 22 builds on that definition to calculate the new position of each airfoil on the new centerline.

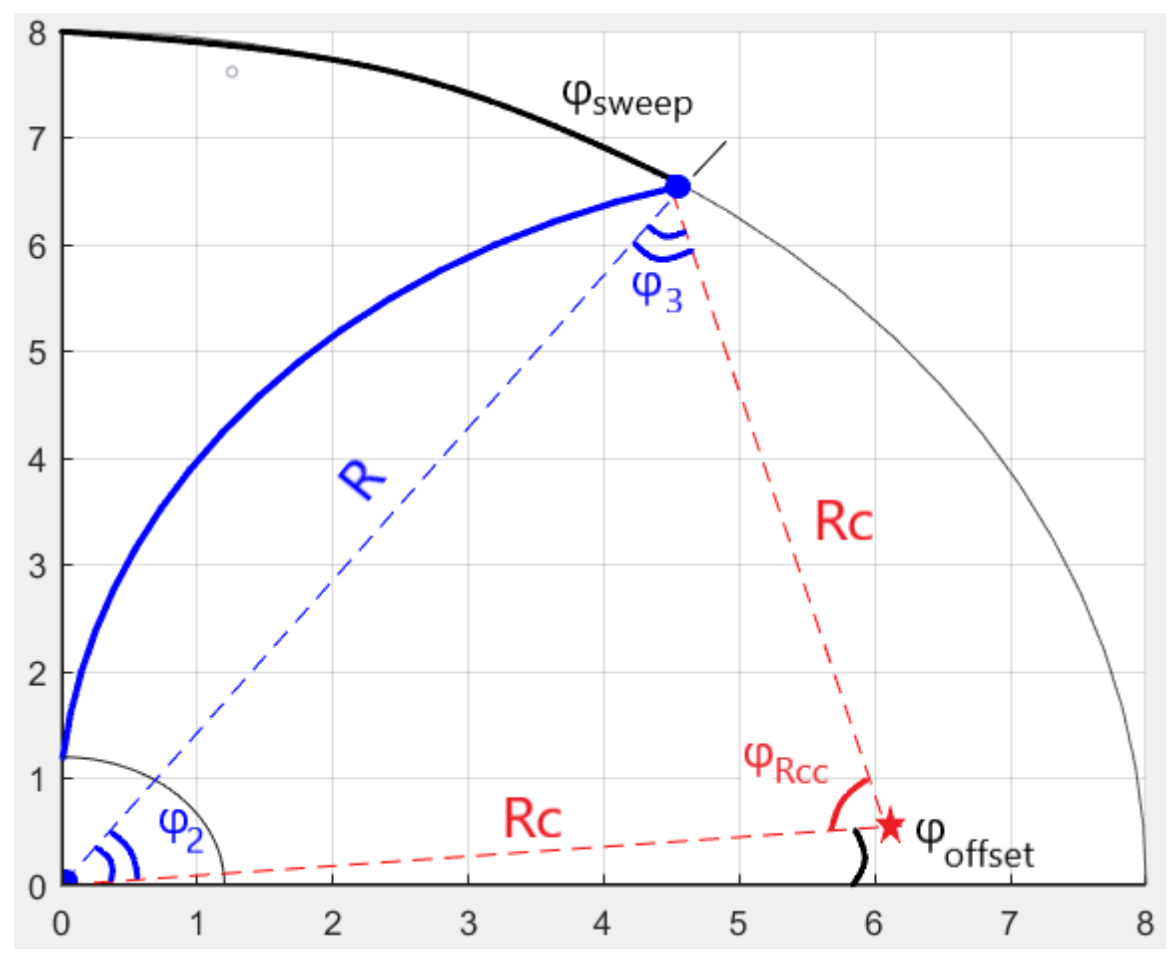

Figure 22: A top-down view of the Geometry for an 8 -inch propeller with a 35-degree sweep. This figure provides an illustration for deriving equation $23-25$.

The first variable that needs to be calculated is the angle of RCC, $\varphi_{\mathrm{Rcc}}$, in red. With all the distances along the isosceles triangle known, the law of cosines can be used to calculate any angle of interest.

$$
\varphi_{R c c}=\cos ^{-1}\left(\frac{R^{2}-R_{c}^{2}-R_{c}^{2}}{-2 * R_{c} R_{c}}\right)=\cos ^{-1}\left(\frac{R^{2}}{-2 R_{c}^{2}}+1\right)=80.396^{\circ}
$$

With $\varphi_{\text {Rcc }}$ known, the other two angles can be calculated

$$
\varphi_{2}=\varphi_{3}=\frac{180-\varphi_{R c c}}{2}=49.822^{\circ}
$$


Now the offset angle can be calculated. This angle defines where RCC is placed on the cartesian plane. The offset angle can be related to the input swept angle, $\varphi_{S w e e p}$, and $\varphi_{2}$ since all three angles contribute to the total angle of 90 degrees.

$$
\begin{gathered}
90^{\circ}=\varphi_{\text {Sweep }}+\varphi_{2}+\varphi_{\text {offset }} \\
\varphi_{\text {Offset }}=5.1778
\end{gathered}
$$

With these relationships established for all the variables in figure 21 , we can solve for each intermediate swept angle. Continuing with this example, the steps to calculate the intermediate sweep angle for the airfoil position at $R_{a} / R=0.65$ are calculated below with an accompanying illustration in figure 23 .

$$
\begin{gathered}
\varphi_{2_{-} i}=\cos ^{-1}\left(\frac{R_{c}^{2}-(0.65 * R)^{2}-R_{c}^{2}}{-2 *(0.65 * R) * R_{c}}\right)=\cos ^{-1}\left(\frac{(0.65 * R)}{2 R_{c}}\right)=65.206 \\
90^{\circ}=\varphi_{\frac{R_{a}}{R}=0.65}+\varphi_{2_{i}}+\varphi_{\text {Offset }} \\
\varphi_{\frac{R_{a}}{R}=0.65}=19.616
\end{gathered}
$$

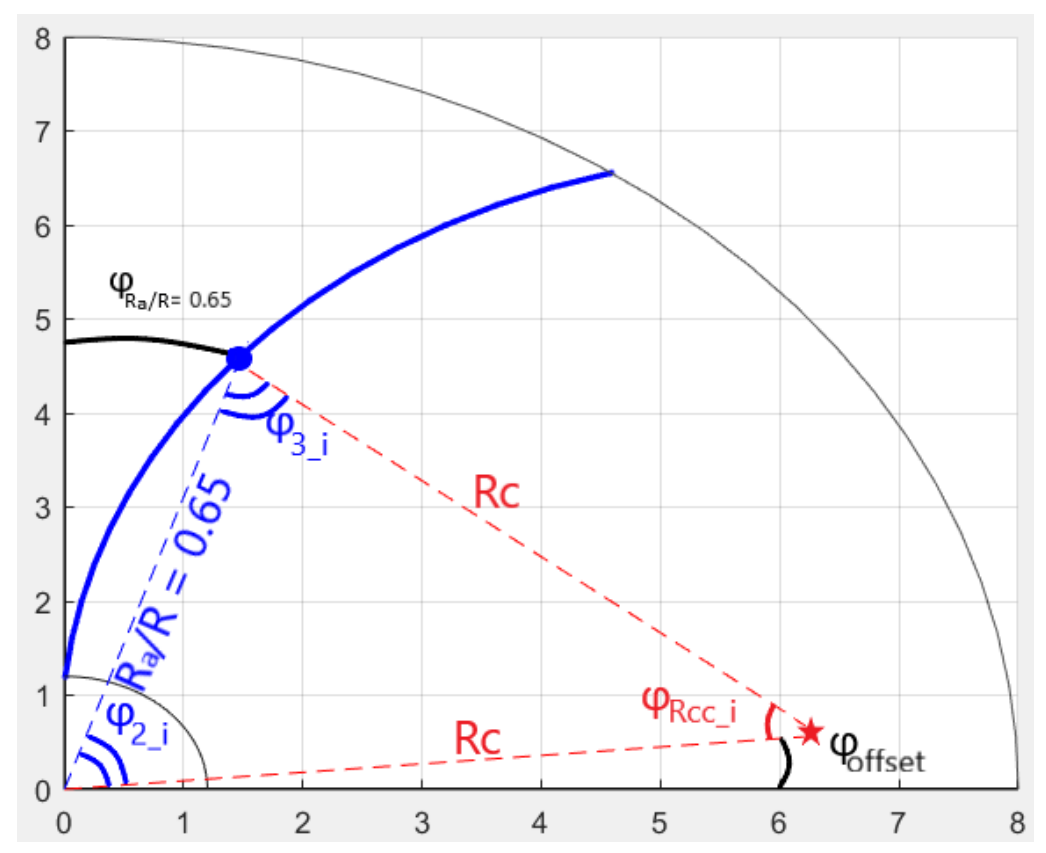

Figure 23: The Geometry required to calculate the intermediate swept angle at $R a / R=0.65$.

This process is repeated for the rest of the airfoils. A table has been constructed for the swept-35 propeller, which is established in Table 13, Appendix B. With the new centerline shape and the airfoil's position defined, one final step is required to construct the swept propeller. The orientation of the 
chord must lie perpendicular to the local radius. This naturally occurs on the conventional propeller but not on the current swept propeller. If the chord from the conventional centerline was only shifted to the modified arc, the propeller would not be swept it would be sheared. Figure 24 demonstrates the need to reorientate the airfoils. Figure 24a shows the 35-degree swept centerline, from the previous figures, with the conventional airfoil chords laid out at each $R_{a} / R$ from root to tip. The chords are simply horizontal lines that do not change with respect to the curved centerline. This prevents the airfoil from acting perpendicularly to the flow of air as the blade spins, resulting in all the airfoils operating at a sideslip angle. Figure 24b, which is a 3D mesh built of figure 24a, show the physical shape of the propeller without orientation. The result is a propeller that is sheared rather than swept. The shearing is most apparent at the tip. To fix this issue, the airfoil chords must be reoriented as seen in figures 24c and $24 \mathrm{~d}$. These two figures show the airfoil chords lying perpendicular to the point where they intercept the swept centerline. The steps required to reorientate the airfoils are completed with eq (28) - eq (36). Figure 25 provides a visual aid for the example calculation.

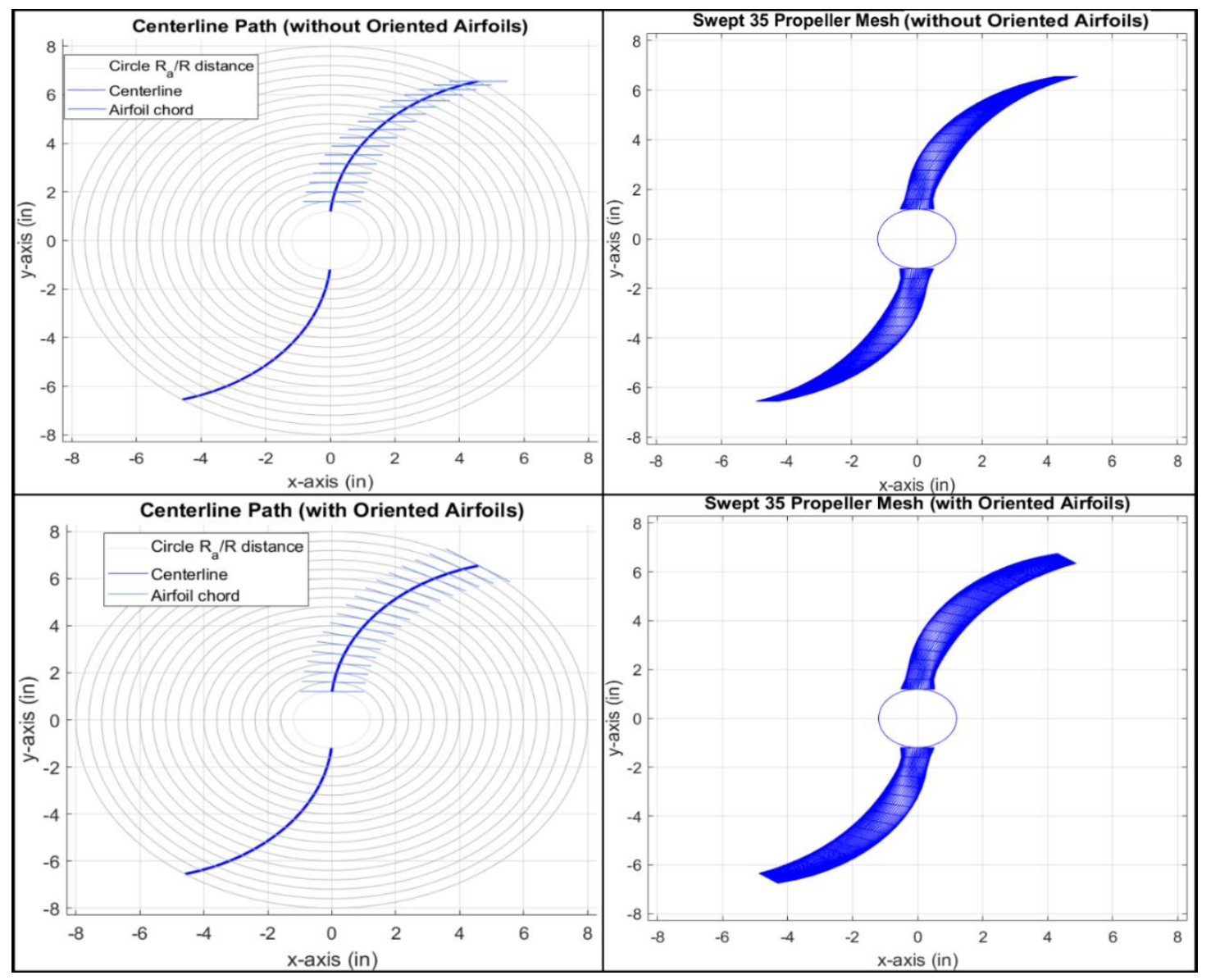

Figure 24a, 24b, 24c, and 24d: The difference between shearing and sweeping a propeller blade. 
The airfoil chords need to be reoriented to be perpendicular to the local intersection. It should be noted that each airfoil will be treated as if it were made of three grid points running from the leading edge to the trailing edge. The leading edge is marked as $\left(X^{l e_{o l d}}, Y^{l e}{ }_{\text {old }}\right)$, the center chord as $\left(X_{c c}, Y_{c c}\right)$, and the trailing edge as $\left(\mathrm{X}^{\mathrm{tr}}\right.$ old, $\mathrm{Y}^{\mathrm{tr}}$ old $)$. To rotate a point around the origin from an old point to a new point, all one needs is equation 28 and an input angle.

$$
\begin{gathered}
X_{\text {new }} \\
Y_{\text {new }}
\end{gathered}=\left(\begin{array}{cc}
\cos (\varphi) & -\sin (\varphi) \\
\sin (\varphi) & \cos (\varphi)
\end{array}\right)\left(\begin{array}{c}
X_{\text {old }} \\
Y_{\text {old }}
\end{array}\right)
$$

However, we are not trying to rotate a point about the origin but instead rotate about some arbitrary position in space. To do this, an arbitrary point in space $\left(X_{\text {arbitray, }} Y_{\text {arbitray }}\right)$ must be subtracted from both sides of equation (29).

$$
\left(\begin{array}{c}
X_{\text {new }}-X_{\text {arbitray }} \\
Y_{\text {new }}-Y_{\text {arbitray }}
\end{array}\right)=\left(\begin{array}{cc}
\cos (\varphi) & -\sin (\varphi) \\
\sin (\varphi) & \cos (\varphi)
\end{array}\right)\left(\begin{array}{c}
X_{\text {old }}-X_{\text {arbitray }} \\
Y_{\text {old }}-Y_{\text {arbitray }}
\end{array}\right)
$$

With equation 29, the orientation point is now with respect to the arbitrary point instead of the origin. Since $X_{c c}$ and $Y_{c c}$ are already positioned where they need to be, the $X_{c c}$ and $Y_{c c}$ are substituted in for the arbitrary points. In addition, the intermediate theta is used to define the rotation angle required for each individual airfoil chord. After placing all known values on the right-hand side, equation (30) can now be used to calculate $X_{\text {new }}$ and $Y_{\text {new }}$ from the $X_{\text {old }}$ and $Y_{\text {old }}$ positions. Note in figure 25, equation (30) rotates the blue line, which represents the airfoil chord, onto the redline making the chord perpendicular to the new centerline.

$$
\left[\begin{array}{l}
X_{\text {new }} \\
Y_{\text {new }}
\end{array}\right]=\left[\begin{array}{c}
X_{c c} \\
Y_{c c}
\end{array}\right]+\left[\begin{array}{rr}
\cos (\varphi) & -\sin (\varphi) \\
\sin (\varphi) & \cos (\varphi)
\end{array}\right] *\left[\begin{array}{c}
X_{\text {old }}-X_{c c} \\
Y_{\text {old }}-Y_{c c}
\end{array}\right]
$$

The airfoil at $R_{a} / R=0.65$ will be used as a demonstration to apply eq 25 . This demonstration starts off where $\varphi_{\frac{R a}{R}=0.65}$ was calculated in equation (24). With $\varphi_{\frac{R a}{R}=0.65}$ known, the position of $\mathrm{X}_{\mathrm{cc}}$ and $\mathrm{Y}_{\mathrm{cc}}$ can be calculated with eq (26) and eq(27). It should be noted that this example uses the $y$-axis as the datum line.

$$
\begin{aligned}
& X c c=R_{a} \sin \left(\varphi_{\frac{R a}{R}}\right)=0.65 * R \sin \left(\varphi_{\frac{R a}{R}=0.65}\right)=1.7457 \text { in } \\
& Y C C=R_{a} \cos \left(\varphi_{\frac{R_{a}}{R}}\right)=0.65 * R \cos \left(\varphi_{\frac{R_{a}}{R}=0.65}\right)=4.8982 \mathrm{in}
\end{aligned}
$$

Next, the airfoil leading edge and trailing edge must be calculated. Since the Xcc and Ycc are at the center of the airfoil and Table 1 gives the chord length, the leading and trailing edge can be calculated. 


$$
\begin{gathered}
X_{\text {old }}^{T E}=X_{c c}+\frac{\frac{C_{r}}{R}}{2}=1.7457+\frac{0.153 * R}{2}=2.3577 \mathrm{in} \\
X_{\text {old }}^{L E}=X_{c c}-\frac{\frac{C_{r}}{R}}{2}=1.7457-\frac{0.153 * R}{2}=1.1337 \mathrm{in} \\
Y_{c c}=Y_{\text {old }}^{T E}=Y_{\text {old }}^{L E}
\end{gathered}
$$

With all the old locations known, the rotation equation will be used to calculate the new point. There are negative signs attached to each angle to rotate the airfoil chord clockwise instead of counterclockwise.

$$
\begin{gathered}
{\left[\begin{array}{l}
X_{\text {new }} \\
Y_{\text {new }}
\end{array}\right]=\left[\begin{array}{l}
1.7457 \\
4.8982
\end{array}\right]+\left[\begin{array}{cc}
\cos \left(-\varphi_{\bar{R}}^{r}=0.65\right. & -\sin \left(-\varphi_{\bar{R}}=0.65\right) \\
\sin \left(-\varphi_{\frac{r}{R}=0.65}\right) & \cos \left(-\varphi_{\frac{r}{R}=0.65}\right)
\end{array}\right] *\left[\begin{array}{l}
1.1337-1.7457 \\
4.8982-4.8982
\end{array}\right]} \\
{\left[\begin{array}{l}
X_{\text {new }} \\
Y_{\text {new }}
\end{array}\right]=\begin{array}{l}
1.1692 \text { in } \\
5.1037 \text { in }
\end{array}}
\end{gathered}
$$

Equations (31)-(36) have only shifted 1 grid point which must be replicated for each airfoil point along the entire airfoil at each station. The reorientation procedure can be automated with two for loops. A MATLAB script has been provided in the appendix which automates the entire procedure based on the users input for tip radius, radius of curvature, and sweep angle.

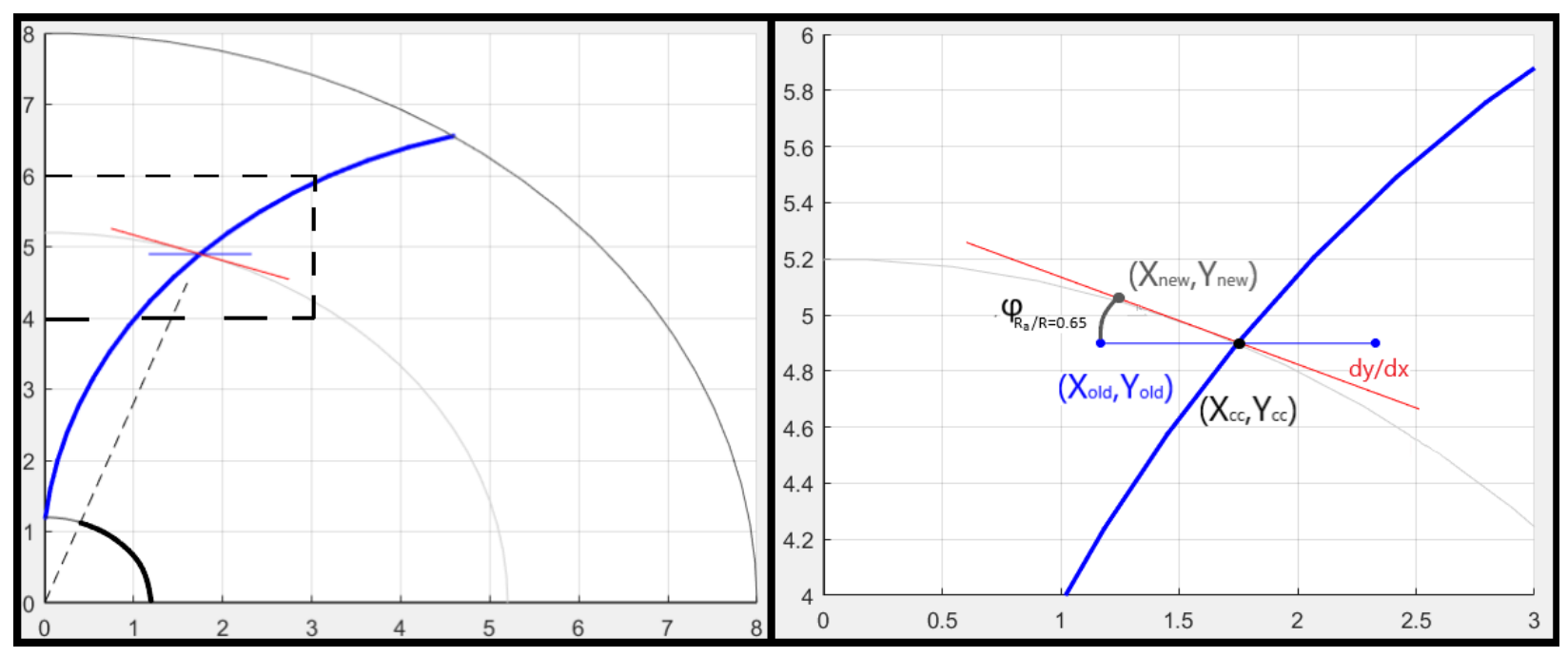

Figure 25a and 25b: The relations ship between the tangent slope for the airfoil chord $r / R=0.65$, in red, and the current airfoil orientation, in blue. 
Figure 26 reveals the final geometry of the swept propeller. The top-down view verifies the airfoil chords have been correctly reoriented for their respective intermediate angles. With all the geometry manipulation complete, the geometric information such as airfoil type, chord length, and Beta can be applied to generate a 3D propeller. A second propeller blade with the same geometry has been made with a mesh to illustrate the physical shape of the 3D geometry. This illustration also serves as a visual inspection to ensure that there are no physical oddities' or errors while generating the swept propeller.

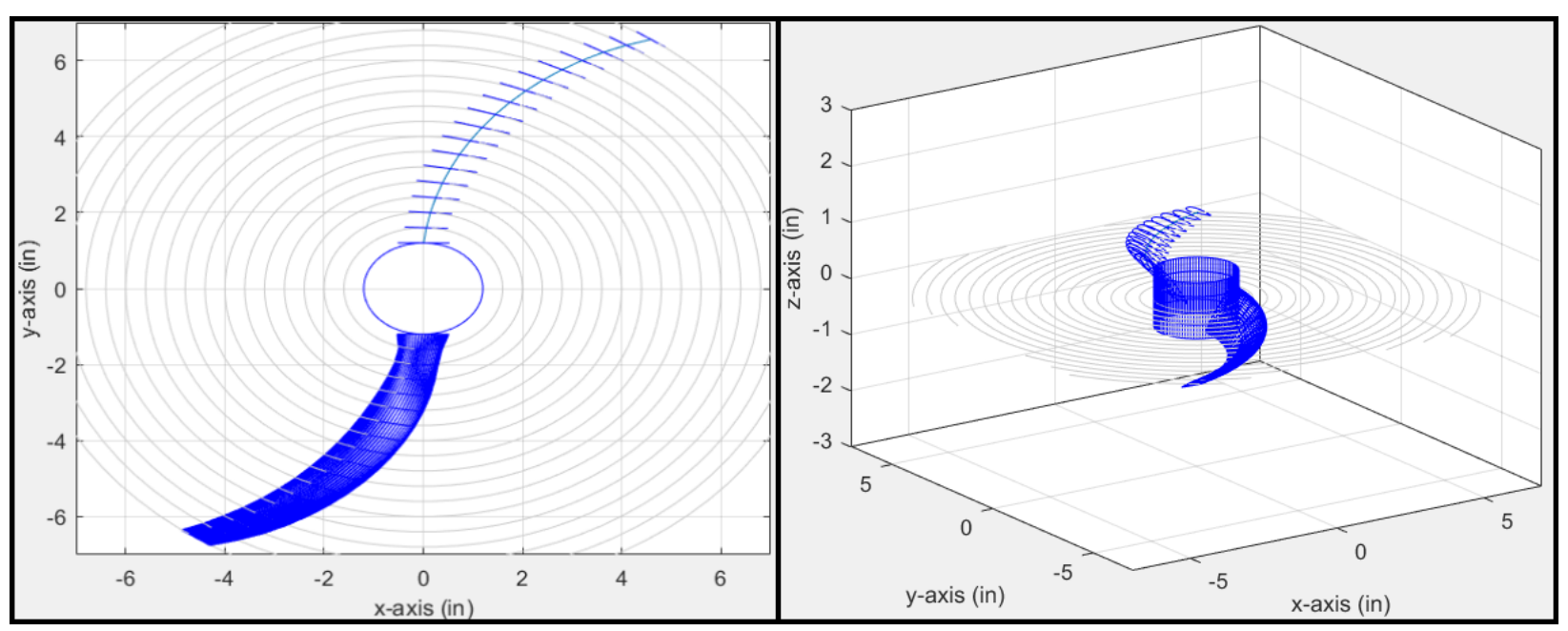

Figure 26a and 26b: Final layout of the three-dimensional $35^{\circ}$ swept propeller viewed from above and the side.

All the new propeller designs will follow a modified naming scheme based on the original diameter and theoretical pitch. The new naming convention will have the standard two parameters as discussed in the Background on Propeller Aerodynamics section followed by the new parameters that defines the sweep. For the Master Airscrew propeller constructed in this example; the 8-inch radius propeller with an 11.636-inch pitch, a 35-degree sweep, and a $0.775 \mathrm{R}$ curvature will be classified as

$$
M A 16 \times 11.636\left|35^{\circ}\right| 0.775 R
$$

This classification is used to define two additional propeller sweeps that will be evaluated both computationally and experimentally. Since both manufacturing and structural integrity are negatively impacted as the sweep angle is driven further and further back and it is ill-advised to manufacture propellers with a sweep greater than $60^{\circ}$. In addition to the complexities of manufacturing the highly swept propeller blades, the circular arc cannot be smaller than $0.5 \mathrm{R}$ because the definition of the RCC point, where the two circles come into contact can only exist if they meet or overlap. If the radius of 
curvature, Rc, circles constructed in figure $20 \mathrm{~b}$ do not overlap, no center point can be defined for the curvature. To demonstrate this limitation, the largest theoretical swept back angle has been generated in figure 27.

Figure 27 shows an 80 sweep, and the appropriate Rc required to ensure the centerline starts at the original centerline and sweeps back until the tip airfoil is 80 degrees off the centerline axis. The Arc value for this sweep meets the criteria to establish an overlapping point for RCC to exist, but the 80 degrees back sweep only just meets this criterion. Even with the centerline constructed, there are still issues with these extreme sweeps. The highly swept propellers require additional information about the airfoil geometry near the tips. Figure 27 a has red lines lying tangent to the curved centerline. These lines represent the slope where the airfoils would normally be positioned. The red lines closer to the root have small slopes that increase gradually from one radial position to the next along the centerline. This allows for a smooth curved propeller shape to form, but near the tip, the variations in slope from one red line to the next start to vary dramatically. This causes a deformation to form at the tips, as seen in figure $27 \mathrm{~b}$. This deformation is due to the lack of grid refinement at the tips.

Figures $28 \mathrm{a}$ and $28 \mathrm{~b}$ compare the propeller shape from the original 18 airfoil stations to a refined set of 30 airfoil stations based on the original 18 airfoils. This shows that larger and more dramatic sweeps require additional geometric information near the tips to properly design a swept propeller. This problem can be solved by interpolating between the already known airfoil positions. The interpolated airfoils, which provide the radial position, chord length, and beta for each new station are automatically generated by XROTOR, when the original 18 airfoil stations from table 1 are written into the program. Even with these additional points, the structural integrity of the propeller is questionable. This propeller was made to demonstrate the maximum potential sweep and will not be considered for further evaluation. 

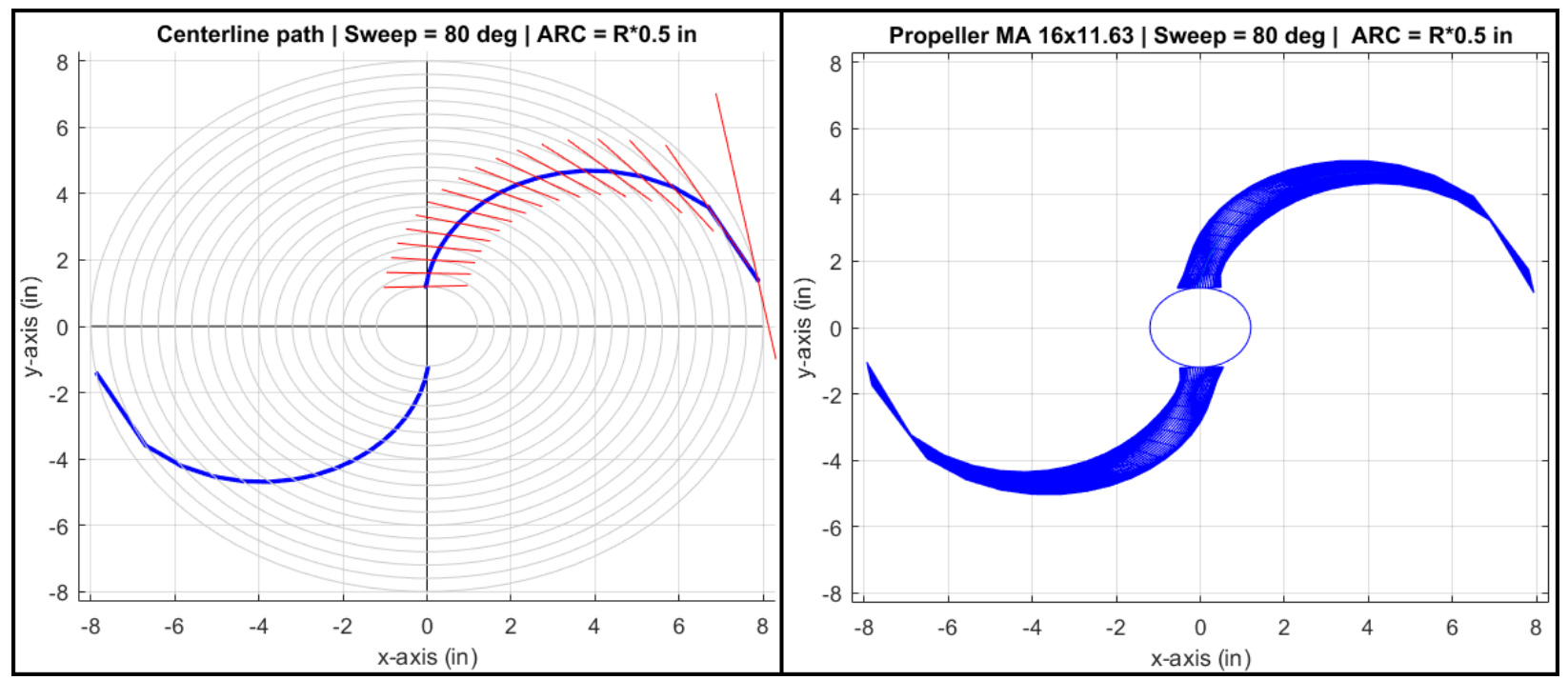

Figure 27: Propeller layout for an 80-sweep
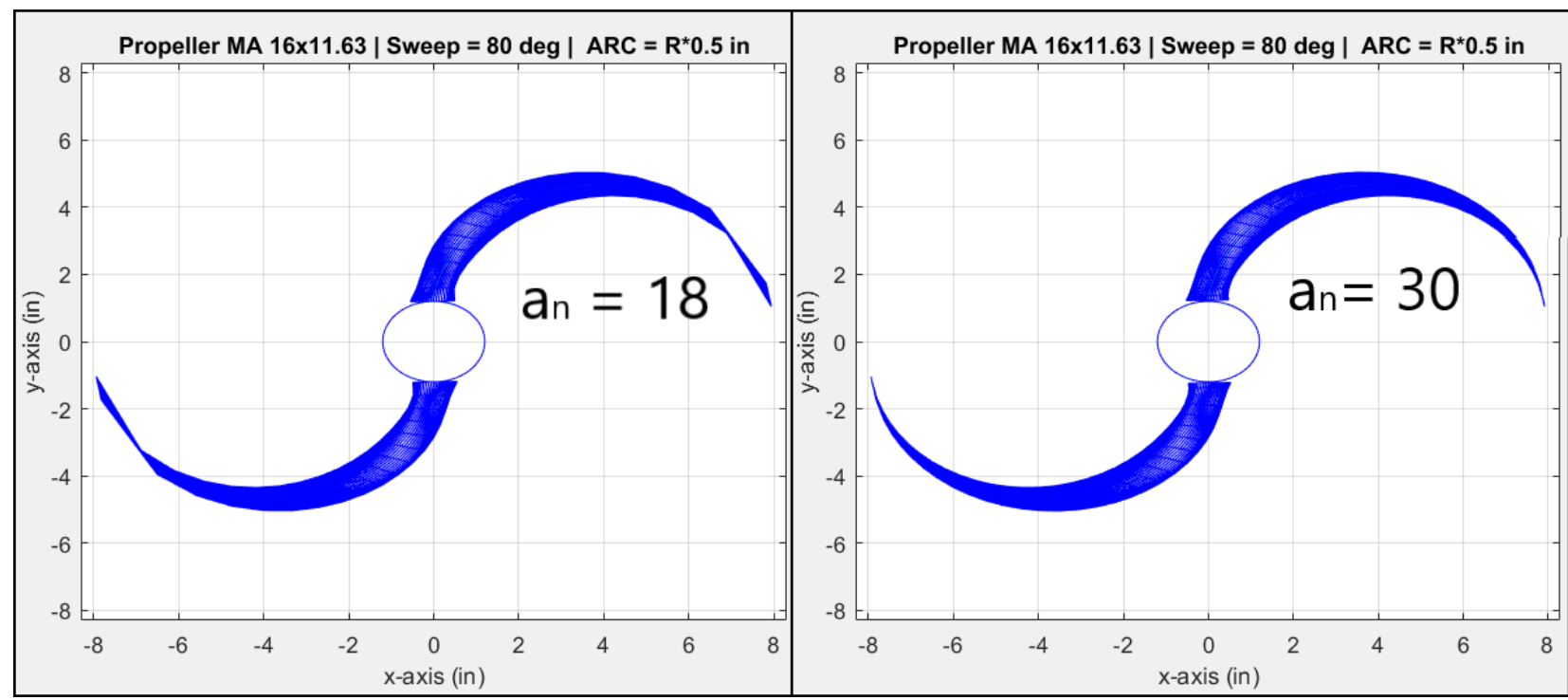

Figure 28: A larger number of airfoil stations need to be defined for larger sweep angles.

The three propeller sweeps that have been chosen for evaluation are the 15, 35, and 55 sweep angles. These sweeps were chosen to provide a test model for the aerodynamic and aeroacoustic performance for small, moderate, and dramatic sweep angles. These models should provide trends demonstrating how the sweep angle affects the coefficient of thrust, torque, efficiency, and sound pressure level. A conventional propeller has been constructed as a control model for comparison against the augmented propeller designs. All the propellers have the same diameter, pitch, chord lengths, betas, and airfoils with the sweep angle acting as the independent variable. All four propeller designs under evaluation are displayed in table 4 and the geometric shape of each propeller can be seen in figure 29. 


\begin{tabular}{|l|l|l|l|l|}
\hline & Diameter (in) & Pitch (in) & Sweep $\left(^{\circ}\right)$ & Curvature (Non-Dimensional) \\
\hline Conventional Prop. & 16 & 11.636 & 0 & 0 \\
\hline Swept Prop \#1 & 16 & 11.636 & 15 & $1.6536^{*} \mathrm{R}$ \\
\hline Swept Prop \#2 & 16 & 11.636 & 35 & $0.775^{*} \mathrm{R}$ \\
\hline Swept Prop \#3 & 16 & 11.636 & 55 & $0.56228^{*} \mathrm{R}$ \\
\hline
\end{tabular}

Table 4: List of propellers that will be constructed.

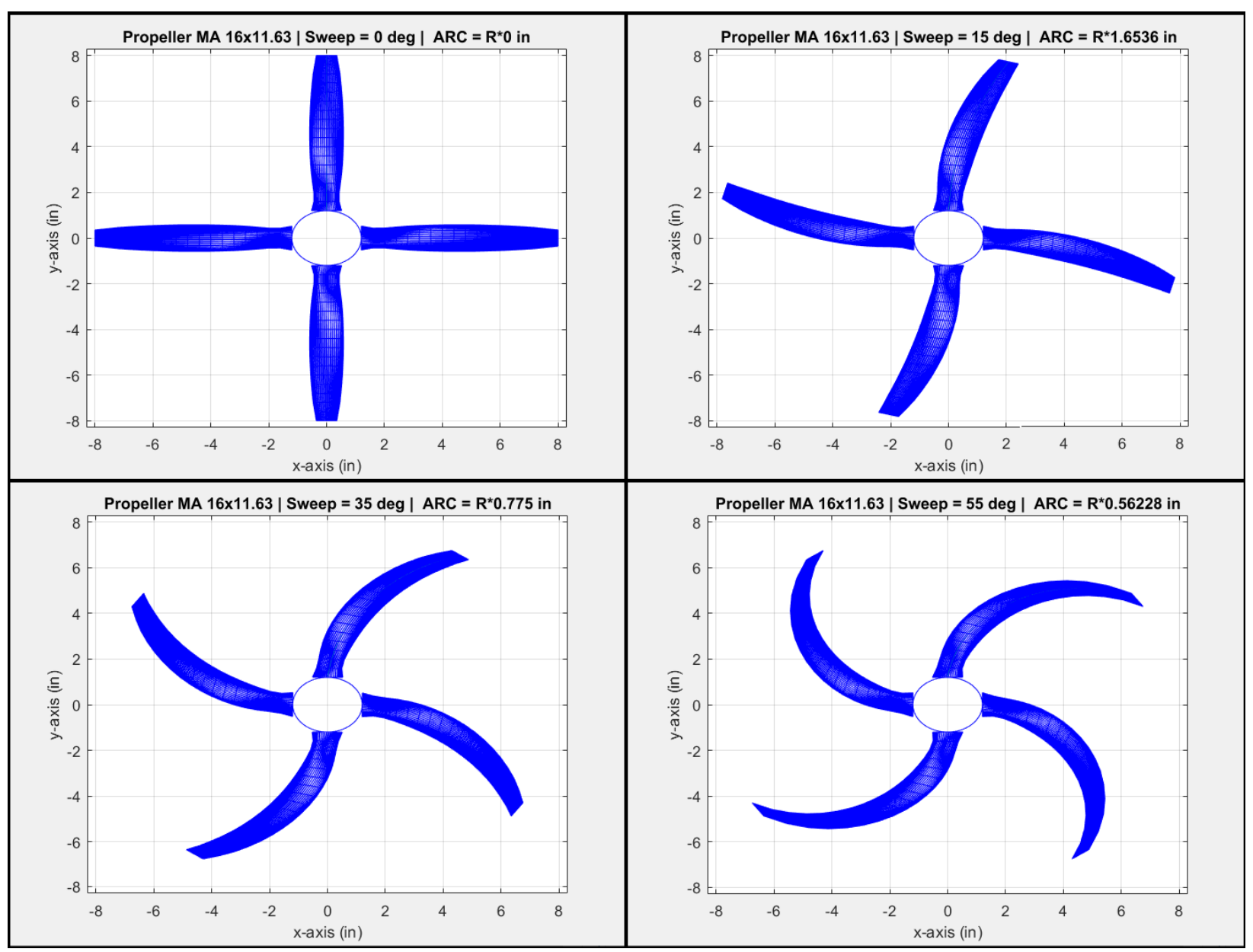

Figure 29: The final geometry of the 4 different types of propellers that were constructed.

The initial work for defining the propeller sweep was done in MATLAB and the script used to generate the shape of the various propeller blades can be found in Appendix A. However, the final design for each propeller was constructed in Inventor with a hub mount. A procedure explaining the step-by-step process to construct the propeller in Inventor can be found in reference 31 . This unpublished document explains, in detail, all the steps required to build both the conventional and swept propeller. This document also explains how to construct all the geometric properties with respect to both the 
propeller's radius and radius of curvature, allowing a user to quickly adjust the inventor model to various lengths and sweep angles. Figure 30 shows the Inventor files for the various propellers with hubs mounts for each design. Table 5 presents the chord length and surface area for each propeller and the relative difference in area. This table shows that, despite the varying chord lengths between each design, the surface area for each propeller is only slightly larger, with the dramatic sweep of 55 only increasing the surface area by three percent.

\begin{tabular}{|l|r|r|r|}
\hline Prop Design & Chord length (in) & Surface Area $\left(\right.$ in $\left.^{2}\right)$ & difference in area \% \\
\hline Straight & 6.8 & 16.442 & \\
\hline 15 Sweep & 6.927 & 16.483 & 0.25 \\
\hline 35 Sweep & 7.493 & 16.647 & 1.25 \\
\hline 55 Sweep & 8.654 & 16.965 & 3.18 \\
\hline
\end{tabular}

Table 5: Chord length and surface areas for each propeller from root to tip.

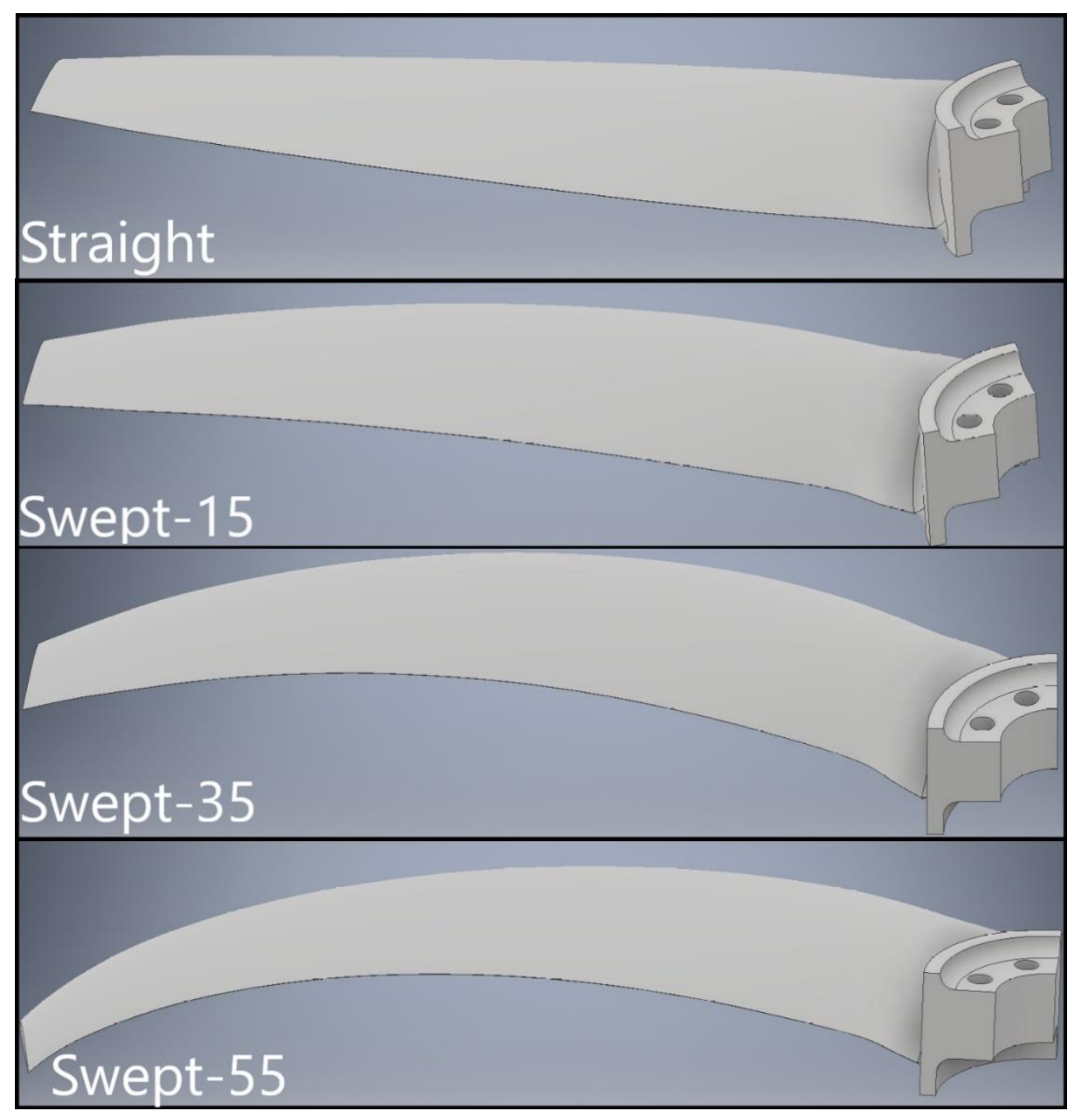

Figure 30: The final geometry, constructed in Inventor, for each propeller. 


\subsection{MANUFACTURING APPROACHES}

Four types of propellers were constructed in Inventor: the straight propeller and the three swept designs. The hubs are designed so the propellers can be mounted to a two, three, or four bladed hub plate. This allows for various blade configurations to be evaluated during testing. A total of 16 propeller (four blades for each design) blades were manufactured out of aluminum 6061 with a CNC milling machine. The milling machine of choice was the Haas Mini Mill 2, presented in figure 31.

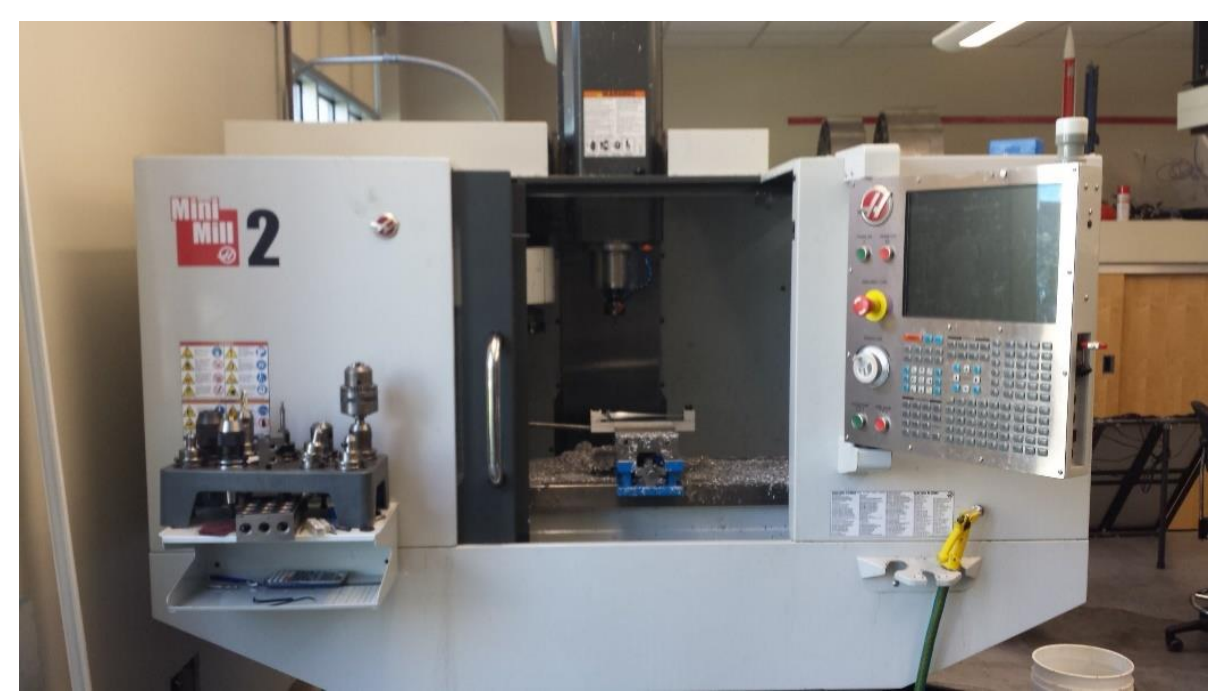

Figure 31: The Super Mini Mill 2. This CNC machine was used to mill all 16 propellers.

Two different milling methods were explored to determine an ideal milling process for the propellers. The first method was the three degree of freedom (3DOF) method. This method used a rotary table to bolt and securely fasten a bar of aluminum 6061 to the rotary table. Once fastened, two milling stages were required to mill away excess from the top and bottom of the bar. The first, initial cut removed large portions of the bar to form the general shape of the blade. The second, finer cut would mill the roughed bar into the finalized shape with step sizes of 1/1,000 of an inch to create a smooth finish. These two milling stages are presented in figure 32 with the rough cut on the left side and the finer on the right. After the finer cut was performed, the bar was unbolted and flipped to repeat the process on the underside of the propeller. 


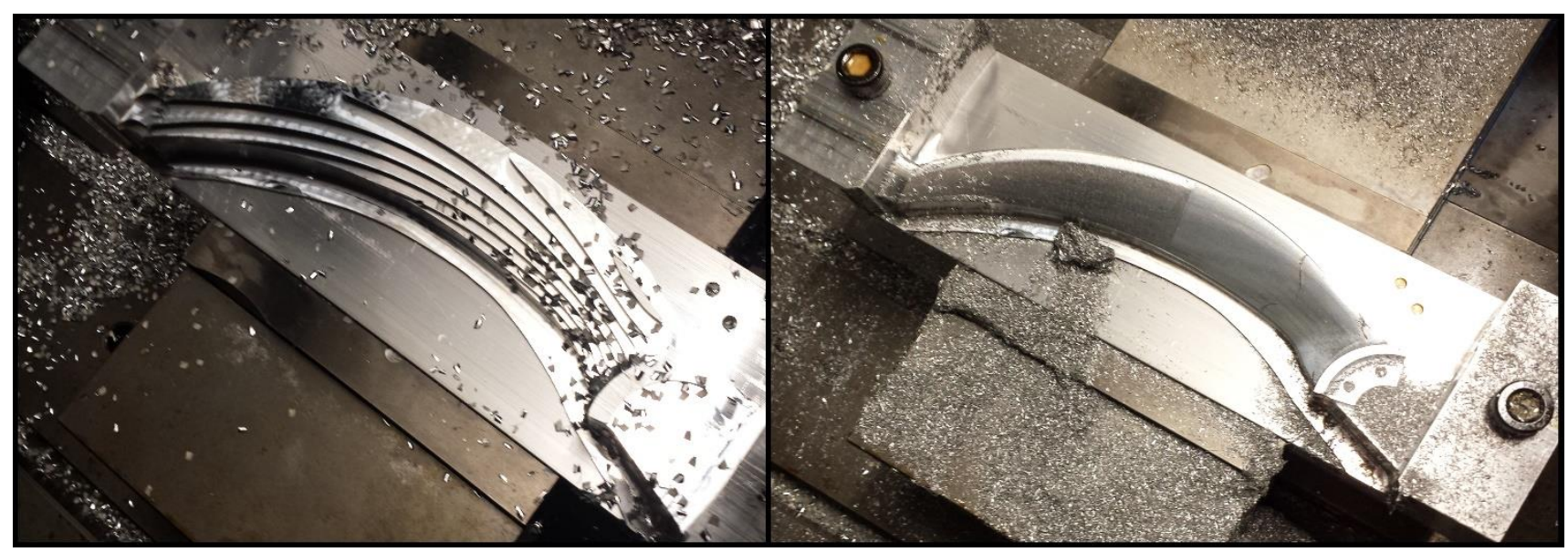

Figure $32 \mathrm{a} \& 32 \mathrm{~b}$ : $32 \mathrm{a}$ presents the 1 st rough cut of milling with the 3DOF method and 32b presents the 2nd finer cut.

With both sides milled, the propeller could be manually trimmed from the supports and any sharp ends would be sanded. This milling process was used to construct four of the swept-35 and swept-55 propellers as well as two of the straight propellers. The blades may appear to have streak lines caused by the milling cutter, but the blade is smooth to the touch. The edges highlighted in green in figure 33 show the support structures, on both ends, that were used to secure the bar during manufacturing. Finally, a light amount of sanding was applied to the tips to ensure the end had a flush surface. This method provided a consistent quality and a smooth finish for all the propeller made with this method. These propellers had to be milled in this manner as the complex geometry would be difficult for the second method, the four degrees of freedom (4DOF).

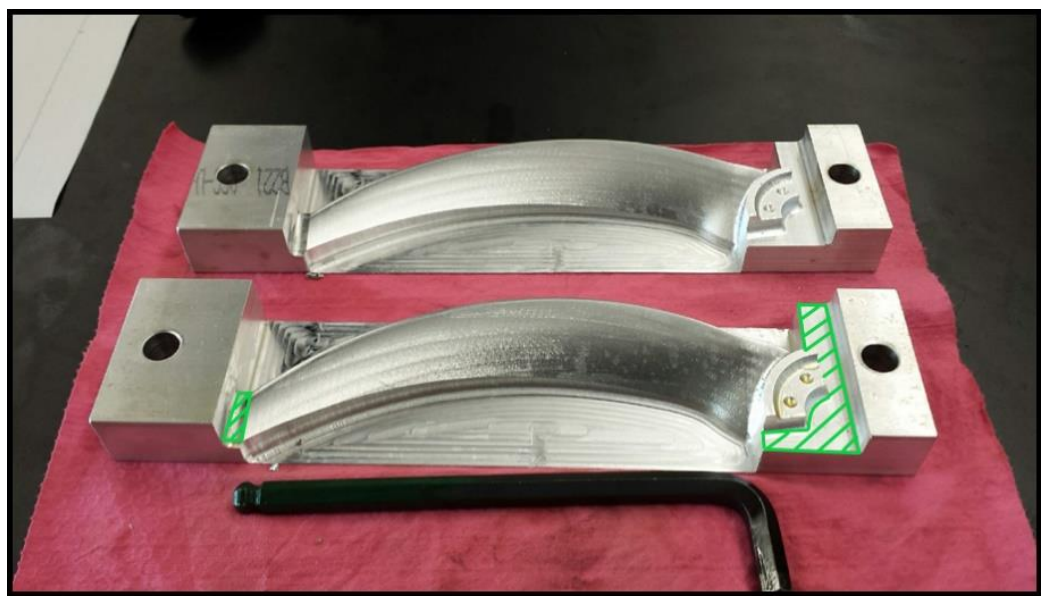

Figure 33: Two swept-35 propellers made in the 3DOF miller with the top side milled away. The highlighted portions would be trimmed after the underside was milled. 
The 4DOF method could be used on the remaining two straight propellers and the four slightly swept, 15-degree propellers as their geometry would not cause interference between the mill and the blade. The 4DOF method relied on a similar technique of performing an initial rough and then a second, finer cut with the bar now supported at the tip with a tail stock and a rotary table providing both a revolving axis as well as a support. The extra degree of freedom allowed the mill to complete the entire process from the solid bar to the finished propeller in one continuous run; however, during the development, two minor issues occurred. The first issue appeared while milling the conventional propellers. A thin, continuous bump along the chord length remained after the process was supposed to be complete, as seen in figure 34 .

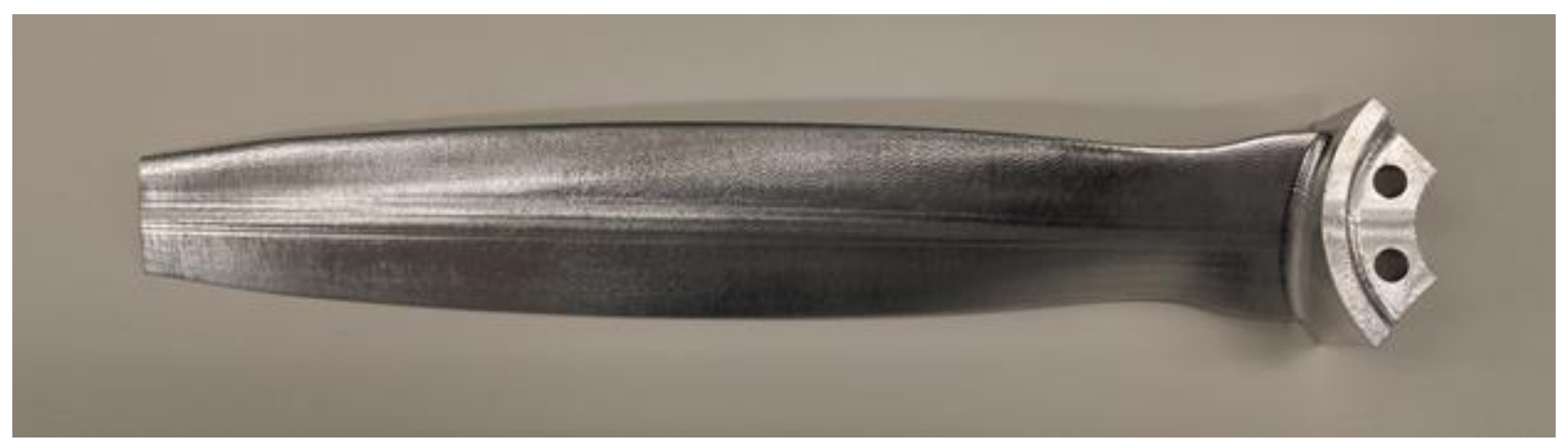

Figure 34: The conventional, straight propeller after milling with the 4DOF method.

This issue was resolved with light sanding, but it should be noted that no significant amount of sanding was not required for the previous milling method. The second issue was observed during the production of the 15-sweep propellers. The first stage worked as expected, cutting away excess and producing the basic shape of the propeller. However, the propellers would flex as the second, finer, stage of the milling occurred, resulting in minor defects that were not seen during production of the more complex geometries. The minor defects can be seen in figure 35 . 


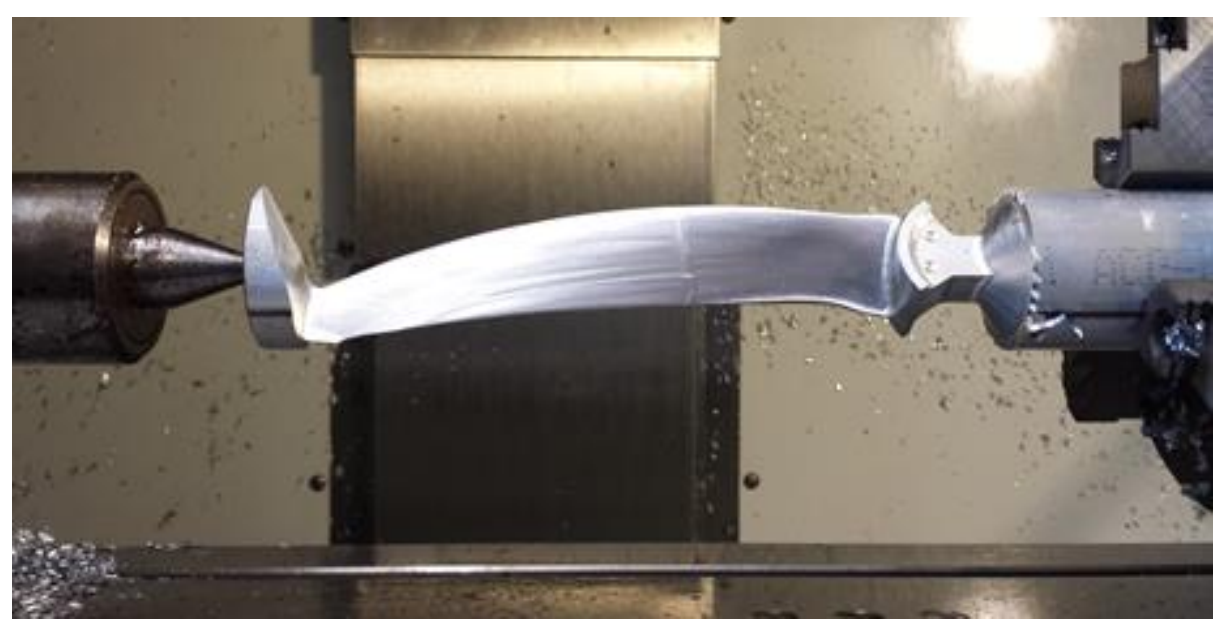

Figure 35: The swept-15 propeller held in with the tail stock and rotary table for the 4DOF milling method.

A test was performed to investigate the cause of these defects, which can be seen in figure $36 . \mathrm{A}$ finished propeller was coated in red layout fluid from root to tip and an additional fine cut was performed to determine where the mill was and was not contacting the blade. Note that only the midsection of the propeller remains coated in figure 36 . This shows that the mill has already performed the proper cut along the surface of the middle section, since the milling tip has "missed" this section. The interior region, between root and the halfway point of the blade, has been stripped of the layout fluid but retains a slightly thicker airfoil. This means, the material was not sufficiently milled because the root was the last section to receive the fine trim and at this point in the milling process, the propeller was a thin beam. Thus, rather than stripping, the propeller deflected causing the interior section to miss the final cut. The deflection was likely amplified by the mounting system, which held the aluminum bar with a tail stock and a rotary table. This platform was not ridged enough to sufficiently secure the propeller during the final manufacturing stage. The 15-sweep propellers required additional sanding to meet satisfactory standards, but this test shows the newer 4-DOF method was not a viable option for consistency or quality. The previous 3-DOF method, while more time consuming, is the superior method to manufacture both the propellers. 


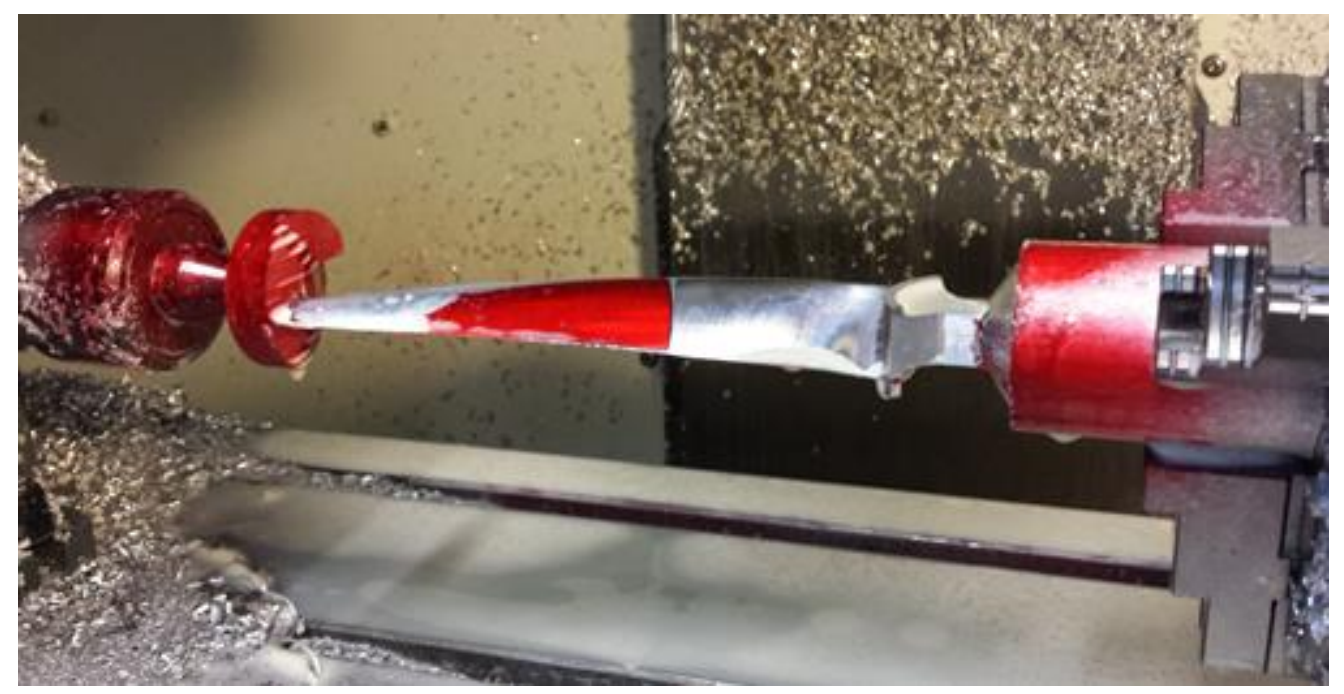

Figure 36: One of the swept-15 propellers used to test for production errors.

After all the propellers were milled, they were lightly sanded with 1000 grit sandpaper to ensure the smoothness between the various designs was consistent. Figure 37 shows all 16 propellers that were milled. With construction completed, the aerodynamic performance of each propeller design could be evaluated.

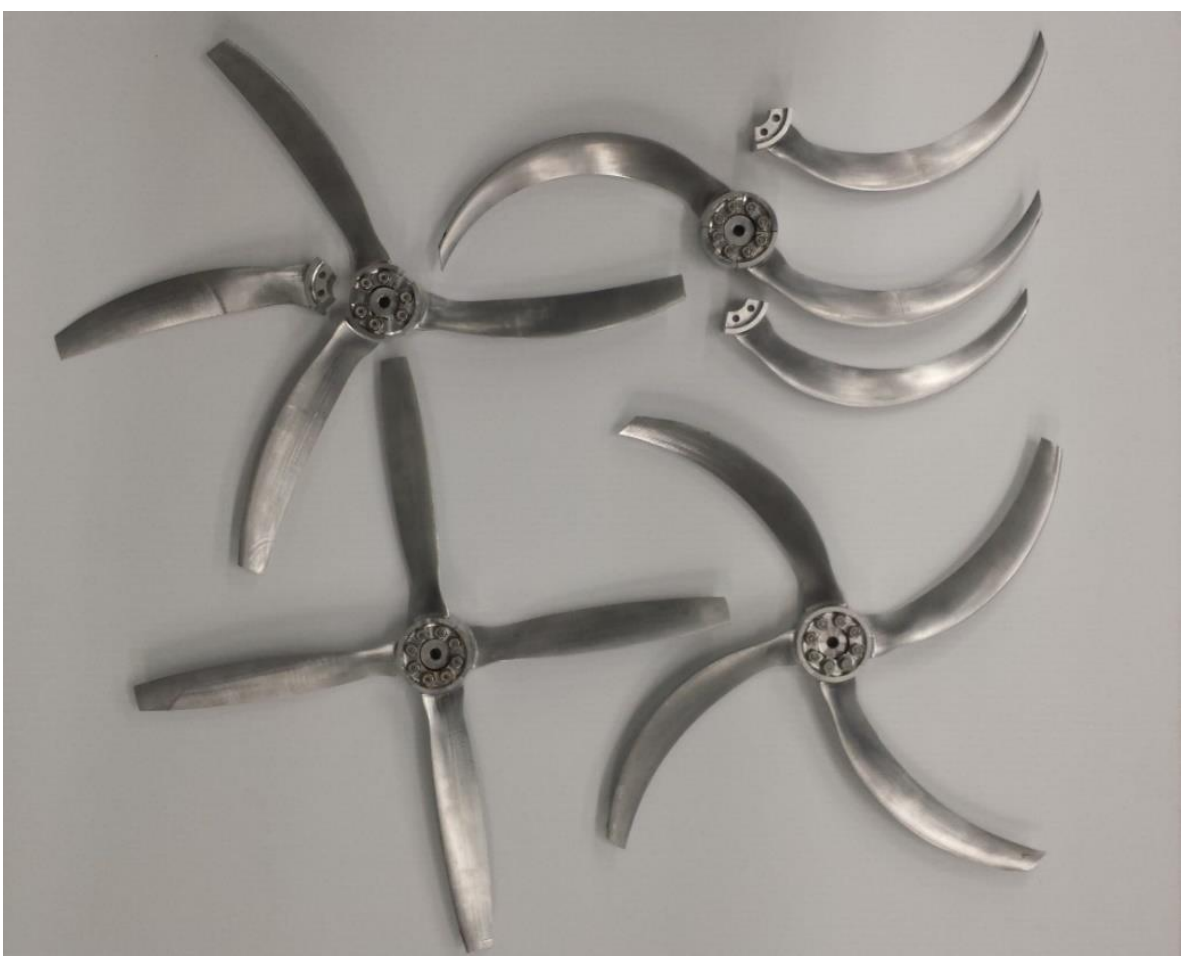

Figure 37: All 16 manufactured propeller blades. 


\section{CHAPTER 6}

\section{EXPERIMENTAL AND COMPUTATIONAL TESTING}

\subsection{ODU WIND TUNNEL}

The aerodynamic evaluation was conducted in ODUs closed-circuit low-speed wind tunnel, which has two test sections. The first section is the low speed $7 \times 8 \times 7 \mathrm{ft}$ section capable of supplying $12 \mathrm{~m} / \mathrm{s}(27 \mathrm{mph}$ ) of steady airflow and the second is the high-speed test section capable of supplying airflow up to $55 \mathrm{~m} / \mathrm{s}$ (123 mph). A basic layout of the wind tunnel can be seen in figure 38 . The propellers were tested in the high-speed section.

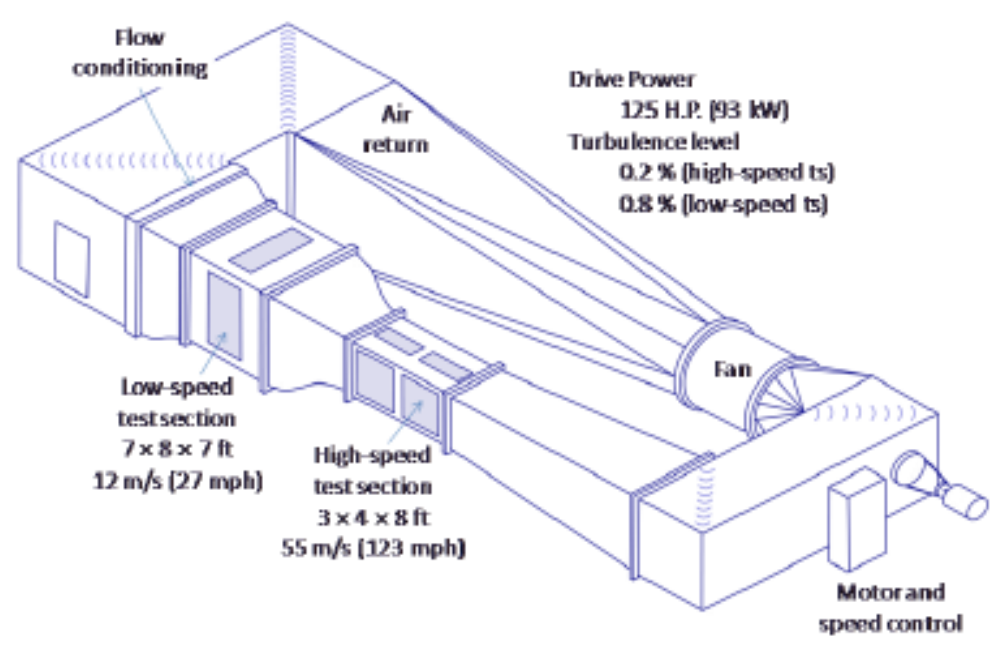

Figure 38: Diagram of ODUs closed-circuit low-speed Wind Tunnel

To collect aerodynamic data for the propellers, a variety of sensors and controls are incorporated into the test stand seen in figure 39 . The test stand uses the ODU $15 \times 15$ load cell to record the forces produced by the propeller [32]. The load cell records how much thrust and torque has been produced based on deformation of the load cell. Other properties such as RPM, tunnel airspeed, and the temperature of the load cell are monitored and recorded for the duration of the experiment. For each propeller, the experiment starts off at a low advance ratio, usually $\mathrm{J} \approx 0.1$, with an increment step of 0.02 or 0.03 . At each increment, the wind tunnel is allowed to come to a steady state before any data can be collected. Once steady, all properties are measured and averaged over a set of 50 points and stored in a spreadsheet. This process of incrementing and measuring is repeated until thrust drops to zero (commonly known as wind milling); this usually occurs around $\mathrm{J} \approx 0.7$. Then the airspeed is dropped 
back to zero to start the process again with another propeller or a different RPM. Before and after each test the balance is monitored to ensure the tare readings did not shift during the experiment.

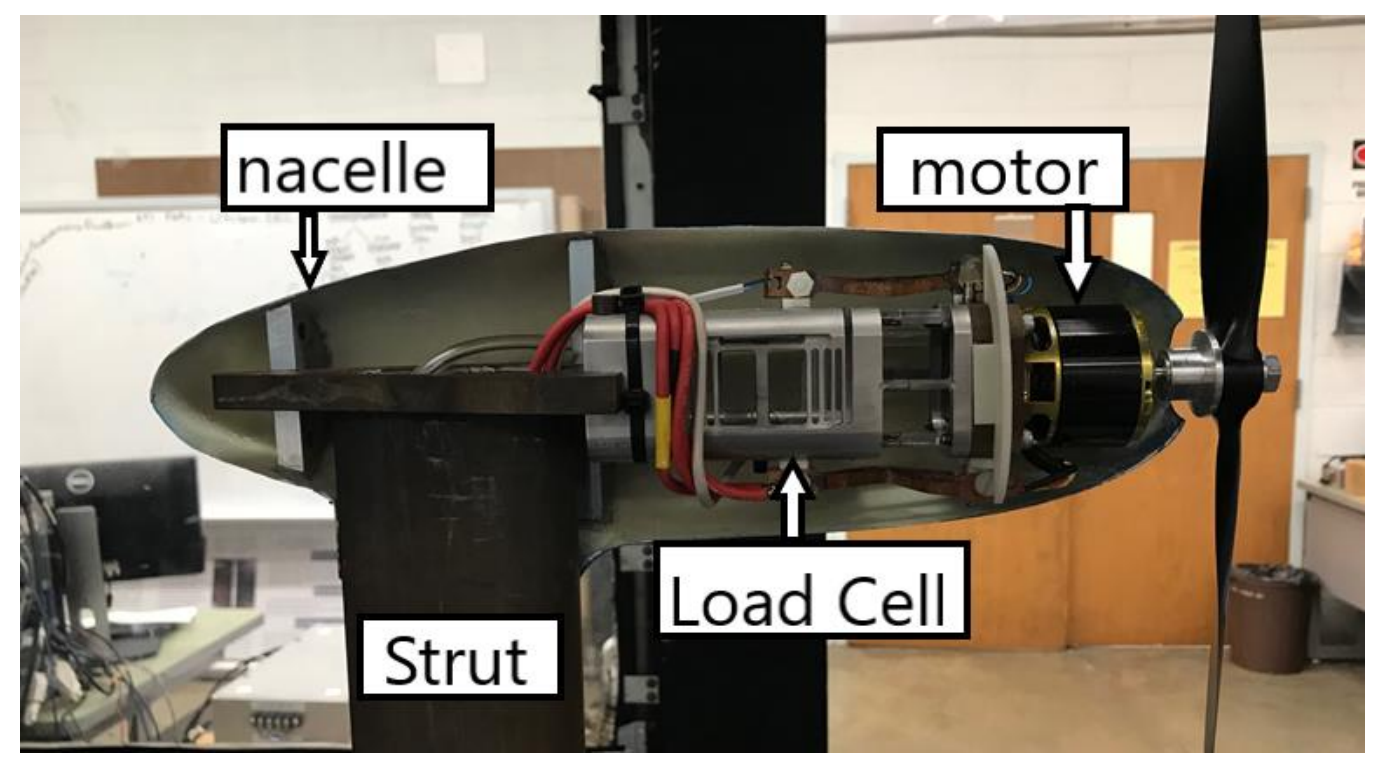

Figure 39: Compartmental view of the test stand and the sensors.

\subsection{OUTDOOR EXPERIMENT}

The acoustic experiment was performed to evaluate the noise signature over a single RPM at static conditions $(\mathrm{J}=0)$. A propeller test stand was constructed to fix a propeller at a desired distance off the ground. A pole was used to fix the propeller in the air without impeding the flow. The structural support was made of lumber and designed to be quick to assemble and disassemble. The stand was built to have a "flat" side to reduce the reflection caused by the stand during operation. The propeller stand can be seen in figure 40. The "flat" side is positioned towards the microphone and is used to collect the acoustic pressures over a duration of time. The microphone used in this experiment was the G.R.A.S. 40PR positioned nine diameters away from propeller at $45^{\circ}$ angle below the plane of rotation. 


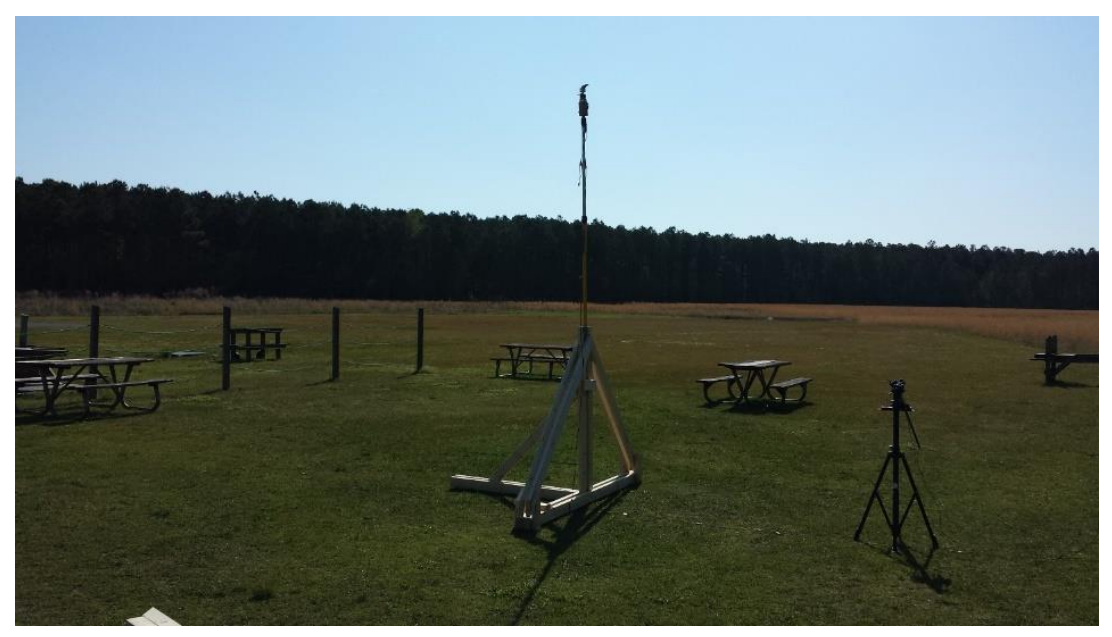

Figure 40: Outdoor test stand

Due to the nature of testing outdoors, it was inevitable for some noise to be introduced from the environment from both weather and ground scattering. To reduce ground scattering, extensive effort would be required to apply acoustic treatment to the area surrounding the propeller stand. This could not be done under the constraints of the study, but the influence of the weather could be minimized. The winds are calmest in the morning before the sun has sufficiently heated up the atmosphere. This provided the best timeframe to run the outdoor acoustic test. However, this means the test could only be conducted for a brief span of time before the winds sufficiently picked up. In addition to running the test on a calm morning, the experiment must be conducted far from any source of community noise. The test would be conducted in a remote, open field. The site chosen to conduct the test was the Newport News, RC park. The facility offered the ideal condition for testing.

Due to time constraints, only the two bladed configurations were evaluated for each propeller design. Other cases such as background noise and motor-only were recorded as well. The motor-only condition required one run under calm conditions, while the background noise was recorded frequently throughout the duration of the morning. This provided a method of monitoring the background noise to determine how significant the ambient noise was relative to the propeller runs. For the experiment, a two-bladed propeller would be mounted to the top of the pole and brought up to an RPM of 5550. 5550 RPM is a strange RPM to choose, but this value was selected as a balancing act. Higher RPMs are desirable for reducing the signal-to-noise produced by the propeller and the environment. Higher RPMs ensure the propellers tonal characteristics are apparent in the analysis. However, higher RPMs are more likely to sway the pole if the wind starts to pick up. This RPM value appeared to be an ideal compromise for the experiment. 


\subsection{ANOPP2 SETUP}

Figure 41 presents a basic layout of the computational approach. The propeller rests at the origin with a semi-sphere of observers surrounding the propeller. All these observers are fixed at a distance, $R$, away from the propeller at various azimuth and elevation angles which are represented by $\theta$ and $\phi$ respectively. The observers are located at the intersection of each black line. Each observer will keep an Acoustic Time Pressure History (APTH) file for one complete rotation of the propeller. The observers operate in retarded time, so the APTH file starts when the first acoustic pressure hits the observer and ends once the last wave passes. The APTH files will be used to compare the various designs and determine if there is any appreciable reduction in predicted noise.

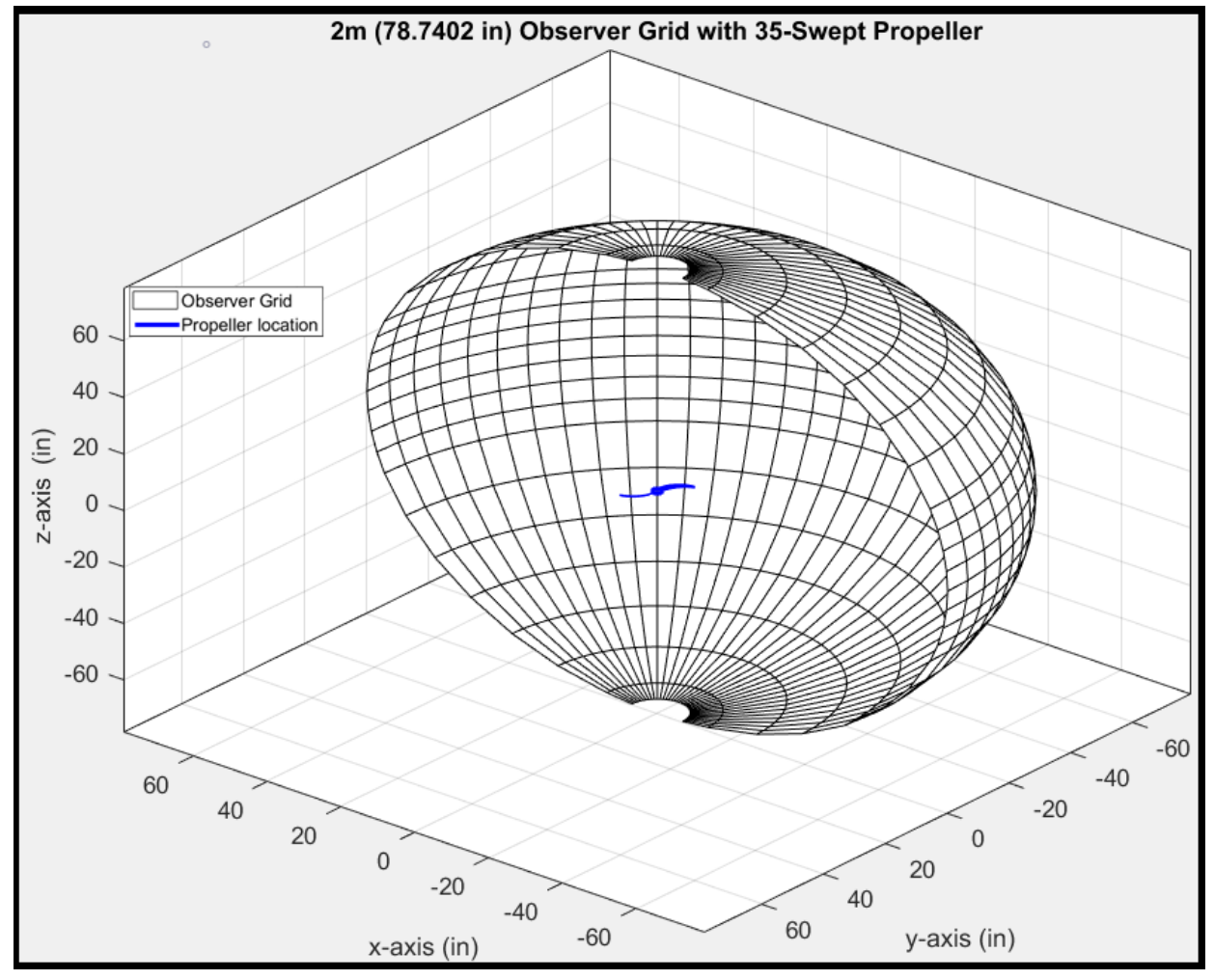

Figure 41: The simulation environment for the ANOPP2 analysis.

The coordinate system for the observer grid can be seen in figure 42 , which also identifies where the azimuth and elevation starts and ends. The green lines indicate where $\theta$ equals 0 and 180 . There are 5degree increments between the two limits spanning the horizon. The red line indicates where $\phi$ equals 5 and 175 degrees. Multiple views have been provided to give a clear outline of the simulation environment. The current observer grid shows a radius of 2 meters (78.7 inches) , but this is just the smallest semi-sphere used for evaluation. In addition to the 2 meter semi-sphere, a $5 \mathrm{~m}, 10 \mathrm{~m}, 20 \mathrm{~m}, 30 \mathrm{~m}$, 
and $50 \mathrm{~m}$ semi-sphere has been constructed for evaluation. Each semi-sphere was used to evaluate the straight, swept-35, and swept-55 propellers at RPMs ranging from 3,500 to 11,000 . Testing and comparing these propellers over a range of distances and RPMs should provide a robust analysis to determine if there are any angles, distances, or RPMs that see a noticeable improvement with regards to the acoustic pressure.

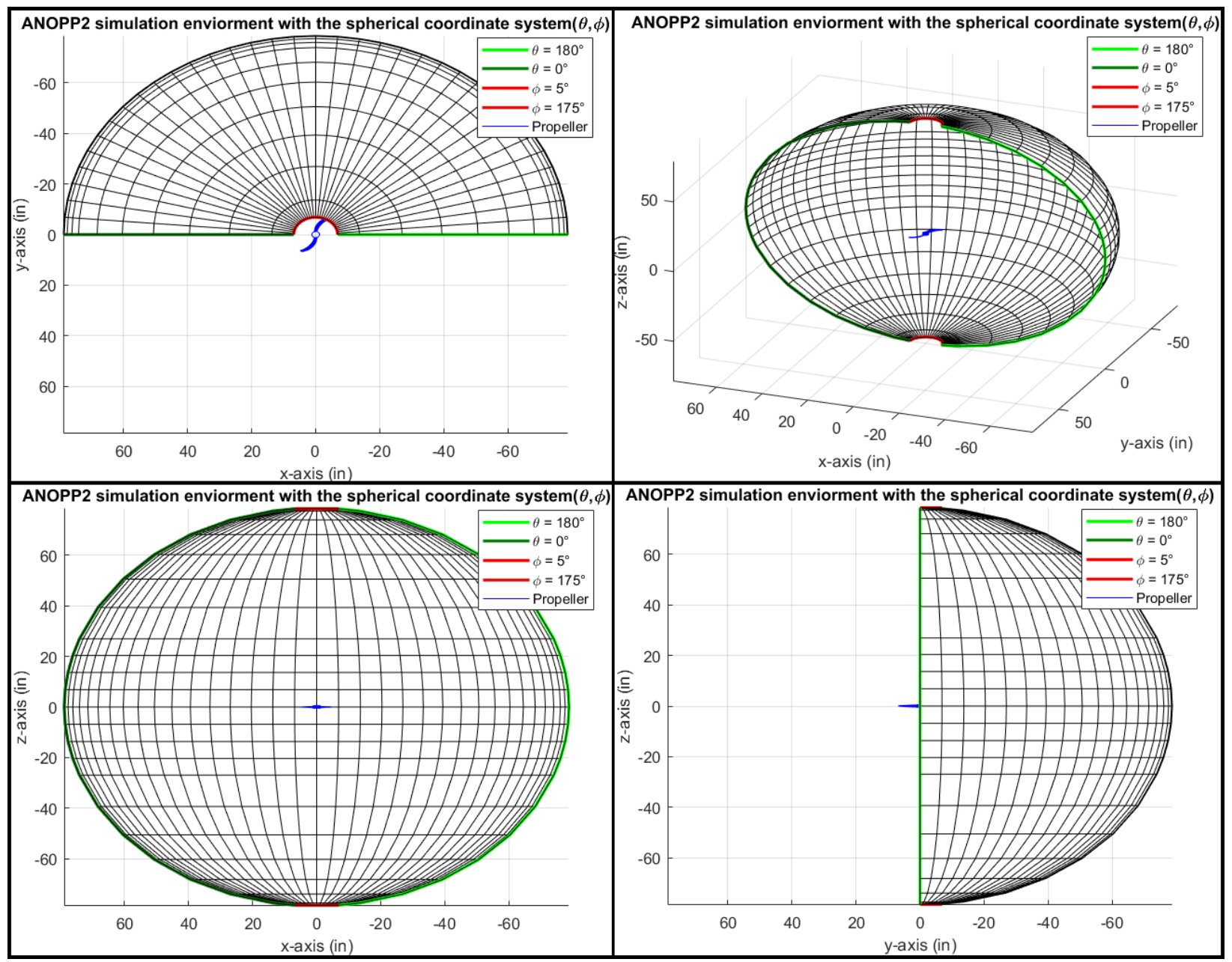

Figure 42: The 50-meter semi sphere showing the spherical coordinate system.

Figures 43a and 43b show how the APTH files are incorporated into the far field. The straight propeller was evaluated at a low RPM of 3500 with the $10 \mathrm{~m}$ semi-sphere. After the simulation was completed, an APTH file was created for each observer. Figure 43a shows the data collected from the observer at $(90,90)$. An acoustic pressure, from a chosen moment in time, was taken from each observer, in this case $\tau=0.0045 \mathrm{~s}$, and added to the grid. The result of selecting and plotting the pressure values from each observer can be seen in figure $43 \mathrm{~b}$, where a pressure gradient has been created as a result of collecting 
all the acoustic pressures for one moment in time. This grid and the contours constructed later, are useful for visual inspection of the results. This allows a user to quickly understand how the pressure changes based on the RPM, distance, and propeller type.

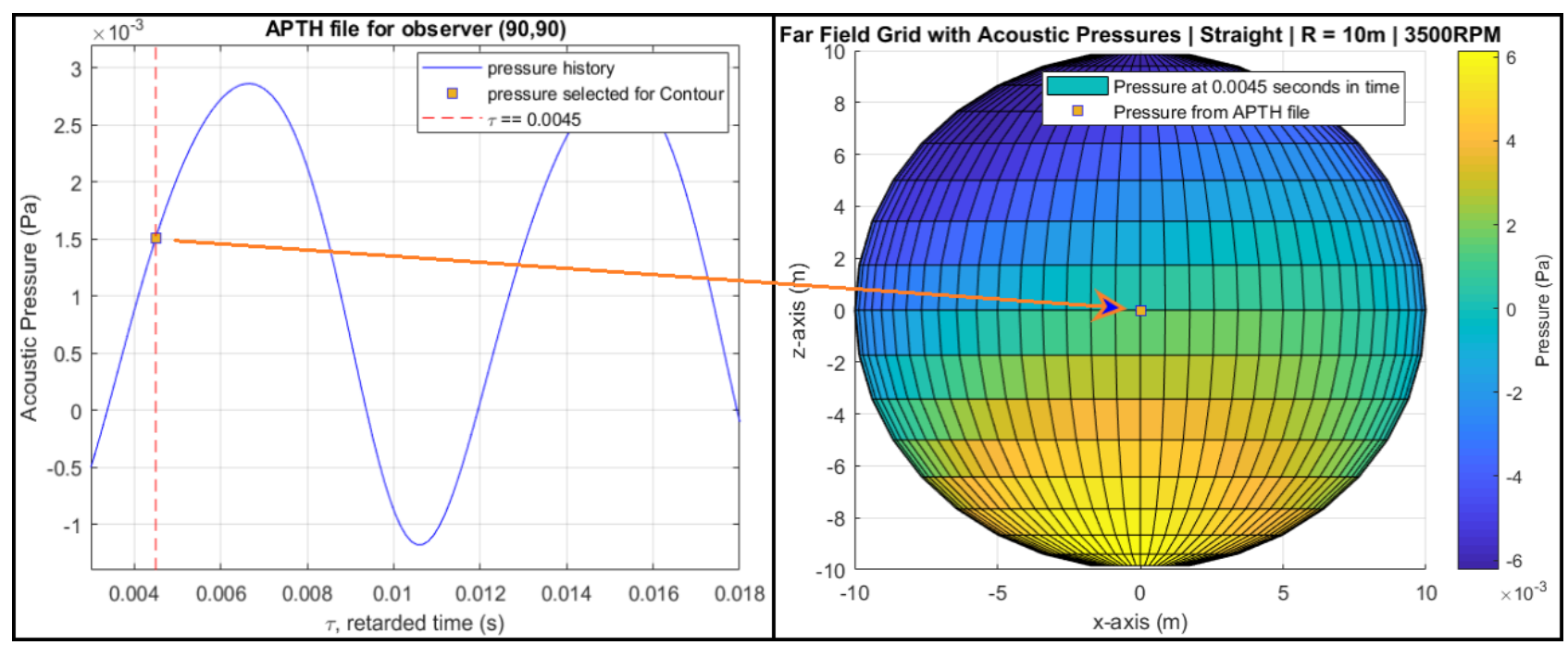

Figure 43: 43a shows the APTH file collected from the simulation. $43 \mathrm{~b}$ shows the far field pressure grid as a result of plotting all pressure when tau $=0.0045 \mathrm{~s}$.

\subsubsection{PROPELLER MODEL}

Figure 44 presents the basic model used to simulate a swept propeller rotating amongst the fixed semi-sphere of observers. Each propeller blade is presented as a lifting line with 30 radial stations. Each node has a coefficient of lift, drag, and airfoil thickness prescribed to represent the aerodynamic and geometric properties. As seen in figure 44, the green and red arrows show a sketch of the coefficient of lift and drag across the propeller, respectively. The aerodynamic properties of interest were evaluated with XROTOR. The NACA 2412 airfoil characteristics were added to the program along with the geometric properties given in table 1 . Since XROTOR does not have any ability to evaluate a swept geometry, a small experimental study was conducted to determine how the aerodynamic coefficients of a swept design differs from the conventional propeller blade. Initial experiments were conducted with Colin Benjamin [33] to evaluate the straight and swept-35 propellers at 3500 and 5000 RPM. The experiment showed consistent overlap between the aerodynamic properties of interest for the two propeller designs. This data suggested that XROTOR is sufficient for predicting the pressure distribution across the propeller for both straight and swept designs. 


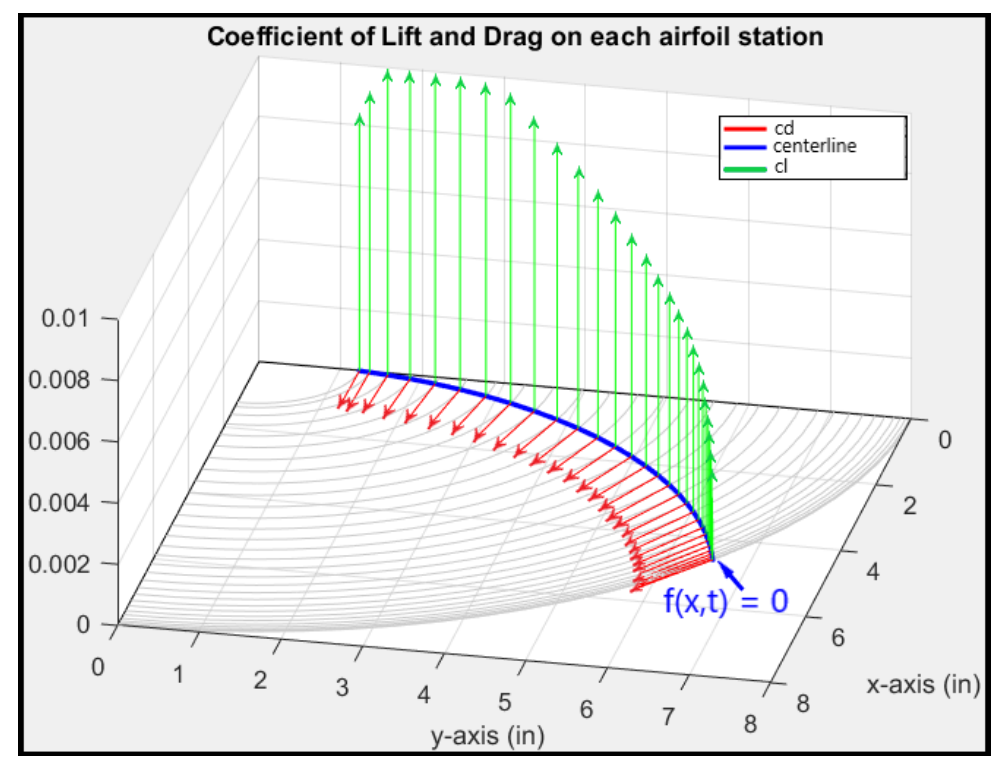

Figure 44: Coefficient of lift and drag on the propeller. The arrows serve as a sketch and not based off the aerodynamic magnitude.

A midrange advance ratio was selected to compare the experimental data against XROTOR. Tables 6-9 show that the relative difference between XROTOR and the wind tunnel is small. With these small difference between the methods, the aerodynamic coefficients for each node could be exported from XROTOR and imported into ANOPP2. With the aerodynamic defined, the only other property needed is the geometry, which consists of the thickness and lifting line shape. The thickness was calculated by treating each NACA 2412 airfoil along the chord line as a 50-sided polygon and calculating the area with MATLAB's built in function, polyarea. The lifting line geometry was calculated with the MATLAB script found in Appendix A. The lifting line, marked as the centerline in figure 44, shows the region where the function equals zero in space and time. This region is where the noise is generated as the line rotates. With all the aerodynamic and geometric information defined, the ANOPP2-F1A analysis can be run to determine the noise signature for each propeller.

\begin{tabular}{|l|l|r|r|r|}
\hline & Properties & XROTOR & Experimental (straight prop) & Error \% \\
\hline Input & RPM & 3500 & 3525.6 & \\
\hline Input & Air velocity (m/s) & 9.19 & 9.19 & \\
\hline Output & Adv. Ratio (J) & 0.38491 & 0.385 & \\
\hline Output & Ct & 0.06797 & 0.0704 & 3.45 \\
\hline Output & Cp & 0.03918 & 0.0413 & 5.13 \\
\hline
\end{tabular}

Table 6: XROTOR data compared to experimental data for the straight propeller. 


\begin{tabular}{|l|l|r|r|r|}
\hline & Properties & XROTOR & Experimental (35swept prop) & Error \% \\
\hline Input & RPM & 3500 & 3500 & \\
\hline Input & Air velocity $(\mathrm{m} / \mathrm{s})$ & 9.07 & 9.07 & \\
\hline Output & Adv. Ratio $(\mathrm{J})$ & 0.38491 & 0.385 & \\
\hline Output & Ct & 0.06881 & 0.0681 & 1.04 \\
\hline Output & Cp & 0.03942 & 0.0396 & 0.45 \\
\hline
\end{tabular}

Table 7: XROTOR data compared to experimental data for the swept-35 propeller.

\begin{tabular}{|l|l|r|r|r|}
\hline & Properties & XROTOR & Experimental (Straight Prop) & Error \% \\
\hline Input & RPM & 5000 & 5000 & \\
\hline Input & Air velocity (m/s) & 12.76 & 12.76 & \\
\hline Output & Adv. Ratio (J) & 0.37677 & 0.38 & \\
\hline Output & Ct & 0.07036 & 0.0686 & 2.500 \\
\hline Output & Cp & 0.03992 & 0.0389 & 2.503 \\
\hline
\end{tabular}

Table 8: Comparison between XROTOR and experimental values for the straight propeller 5000 RPM.

\begin{tabular}{|l|l|r|r|r|}
\hline & Properties & XROTOR & Experimental (Swept 35 Prop) & \multicolumn{1}{l|}{ Error \% } \\
\hline Input & RPM & 5000 & 5000 & \\
\hline Input & Air velocity (m/s) & 11.607 & 11.6067 & \\
\hline Output & Adv. Ratio $(\mathrm{J})$ & 0.3427 & 0.3427 & \\
\hline Output & Ct & 0.0757 & 0.0757219 & 0.029 \\
\hline Output & Cp & 0.04124 & 0.04162 & 0.923 \\
\hline
\end{tabular}

Table 9: Comparison between XROTOR and experimental for the swept-35 propeller at 5000 RPM.

\subsubsection{SOURCE DISTRIBUTION}

With both the thickness and loading noise accounted for, ANOPP2 can now run simulations with the F1A integration equation along the impermeable lifting line. Once the integrals have been evaluated, ANOPP2 will propagate the pressure waves generated by the integration of the F1A formulation from the propeller to the far field observers. Each propeller is treated as a monopole and dipole source superimposed on top of one another, which will simply be referred to as source pairs. Figure 45 shows a conventional and swept-35 propeller propagating to the far-field where the fixed observer records all the acoustic pressures over the duration of the experiment. This figure is simply a sketch meant to convey how a swept propeller's propagation of acoustic waves would differ from a conventional propeller. 

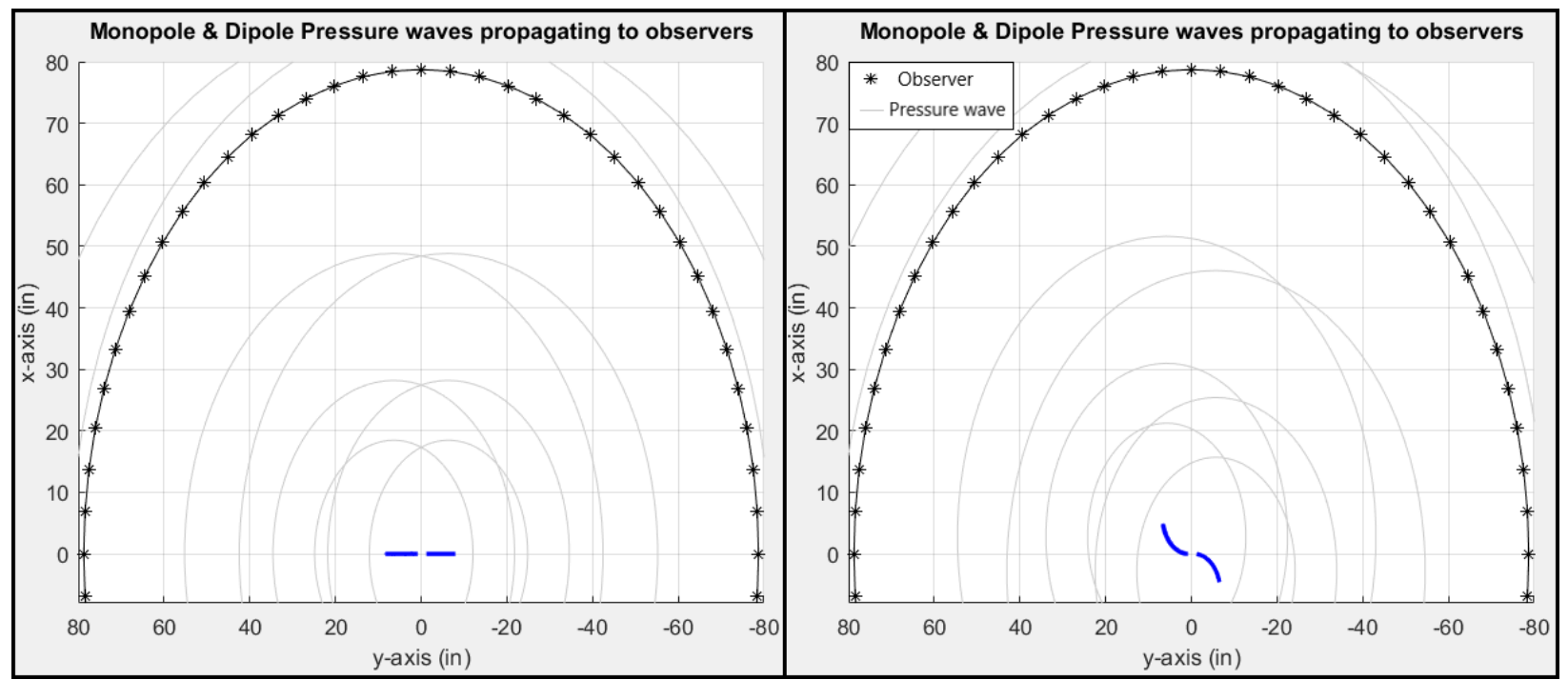

Figure 45: Sketch of the propagation waves from the propeller to observers in the far-field.

However, the swept theory does not treat the entire blade as a single source pair, but a series of monopoles and dipoles distributed throughout the blade. This distribution of sources should better represent the blade's shape and create interference in the fair field. Figure 46 illustrates this concept of interference as an increasing number of source pairs are distributed on a given blade. This interference will be used to evaluate the potential benefits of the swept blade design and the possibility of an optimal shape which would create the maximum interference at a desired distance. The initial interference simulation will consist of six source pairs distributed on a propeller blade with each source integrating over a small segment of the blade instead of one source pair integrating over the entire blade. For example, a propeller is given 30 airfoil sections from root to tip. An integration over the entire 30 airfoil stations would create one source pair for each propeller blade present. For the example in figure 46, the propeller blade was split into 6 segments where segment 1 held the airfoils from station 1 to 5 , segment 2 held the airfoils from station 5 to 10, segment 3 from 10 to 15 and so on until 6 segments were constructed. For figure 46 this would result in a total of 12 source pairs for propagation and interference to be considered. 

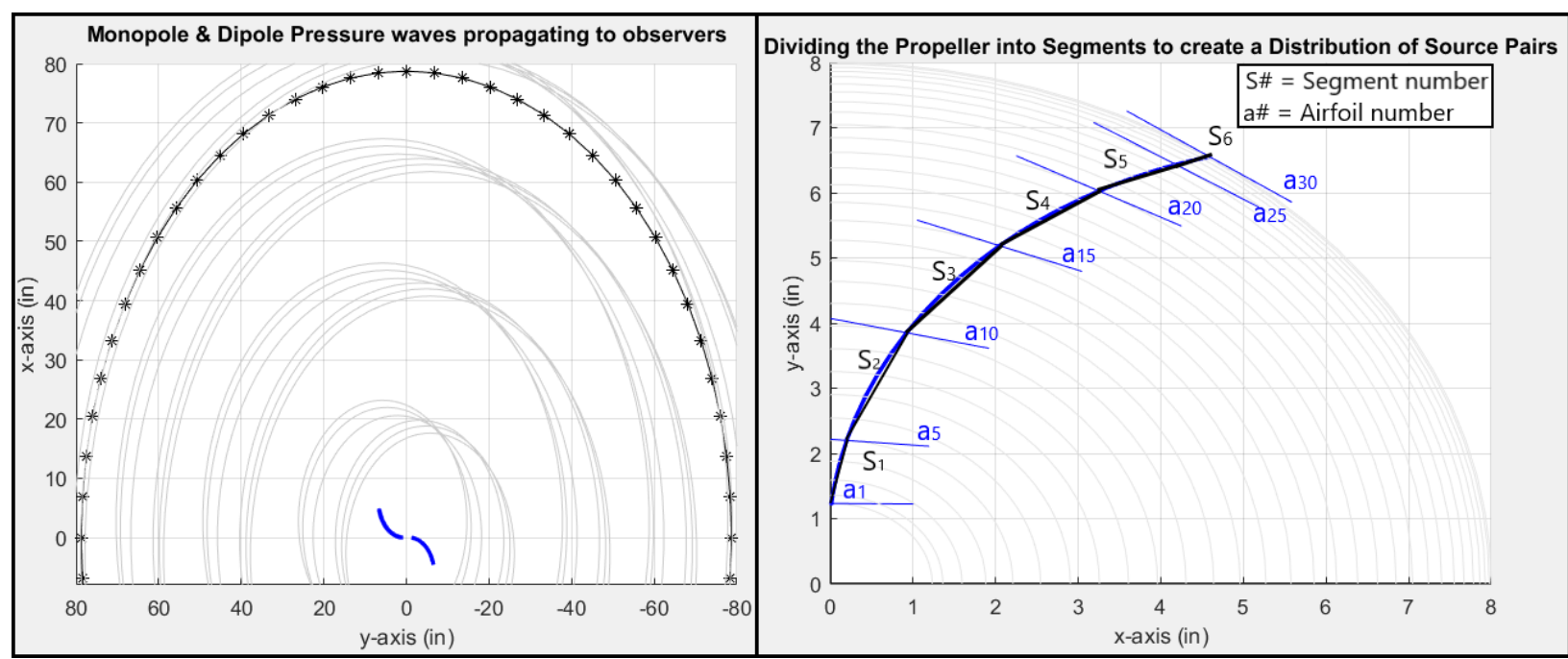

Figure 46: Sketch of a multitude of source pairs propagation to the far-field and the segment division for the propeller.

This could be considered a complex task but ANOPP2 makes the evaluation straightforward. ANOPP2 allows for a "mid source" to be constructed around multiple sources of noise. This "mid source" encapsulates all the individual sources pairs which stores and propagates all the acoustic information to the far field while retaining the constructive/destructive interference and directivity of the individual sources [32]. Figure 47 shows the use of the mid source to encapsulate a series of sources-surfaces produced by the engine and airframe. This information is then propagated to the mid-surface and in turn propagated to the observer as a combination of each noise component; without losing fidelity. Another method is to simply let each source propagate to the observer and combine the sources upon the same time of arrival. In either case, ANOPP2 can make this procedure a simple task.

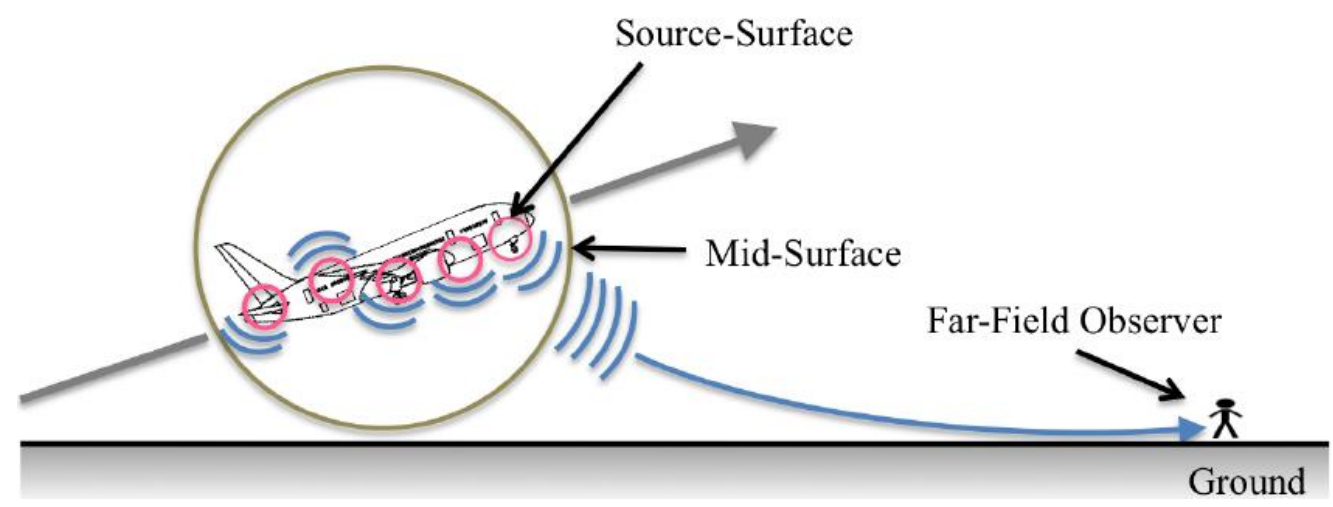

Figure 47: ANOPP2 can combine multiple noise sources and propagate information to the observer with one surface. [30] 


\section{CHAPTER 7}

\section{RESULTS}

\subsection{COMPUTATIONAL RESULTS (ANOPP2)}

A library of coefficients is stored for each RPM calculated in XROTOR. There are five RPM values under consideration, these are 3,500, 5,000, 7,000, 9,000, and 11,000. Each of these five operating conditions will be evaluated in the six semi-sphere simulation environments. In order to run all these scenarios for one propeller design, 30 runs must be conducted. For the three propellers shapes under consideration, this results in a total of 90 runs. This wide swath of acoustic information at various RPM and distances covering all angles should definitively indicate whether a swept propeller could have an acoustic benefit over a conventional propeller. It should be noted that the highest RPM evaluated was 11,000 , because this is the highest speed for a propeller of this size. The tip Mach number is 0.687 and, at these speeds, increasing the RPM would likely create shocks due to local acceleration on the airfoil. Shocks are not represented in this model.

For the first analysis, the straight, swept-35, and swept-55 propeller are tested with two blades operating at 7000 RPM with the observer grid placed at $10 \mathrm{~m}$. All the propellers are initially treated as a source pair for each blade. This test was done to see if there is any stark contrast from the pressure fields produced by the blade. The multi-source propellers will be evaluated afterwards, with the first analysis serving as a direct comparison between the single source pair (two source pairs for two blades) and the multi-source distribution (12 sources for 2 blades). The results from the first analysis can be seen in figures 48,49 , and 50 for the straight, swept-35, and swept-55 propeller, respectively. Each subfigure provides two geometric and two acoustic plots. The top subfigures, a \& b, provide the 3D surface of the propeller being evaluated and the ANOPP2 lifting-line model use in the simulation. The acoustic results of the geometry are shown in subfigures $c$ and $d$. Subfigure $c$ for each initial test shows the semi sphere viewed from the XZ-axis. This is the same perspective as subfigure $42 \mathrm{c}$ where the $\theta$ and $\phi$ angles were defined. However, the acoustic data has been plotted to show how the pressures vary across the far field. Subfigure $d$ takes subfigure $c$ and warps the semi-sphere into a square. This results in a Mercator projection that provides a better view of the edges of the semi sphere but distorts the image. All pressure plots are presented in the units of Pascal ( $\mathrm{Pa})$. 


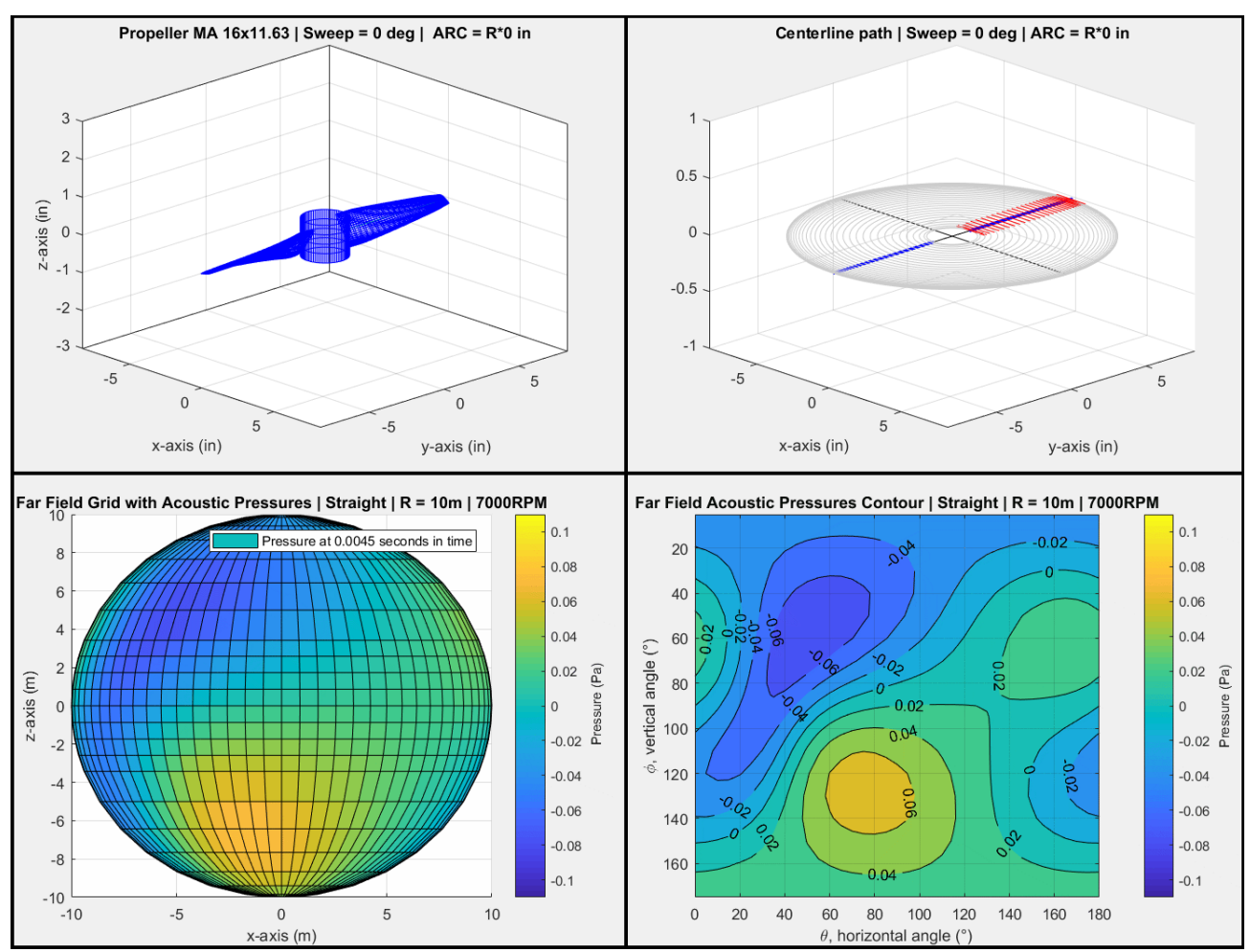

Figure 48: The straight propellers geometry (top) and acoustic pressure output (below) for the 7000 RPM and $10 \mathrm{~m}$ case.

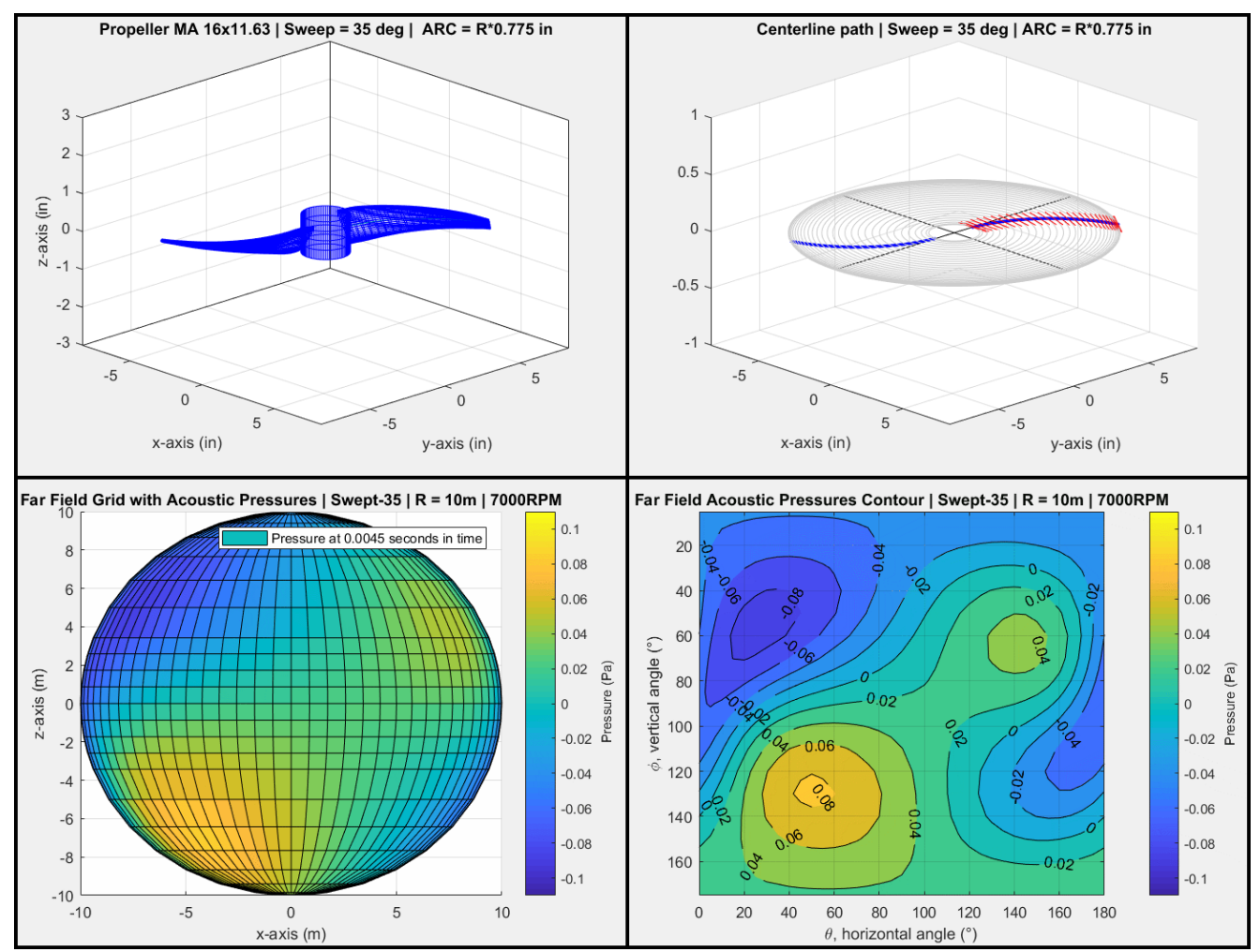

Figure 49: The swept-35 propellers geometry (top) and acoustic pressure output (below) for the 7000 RPM and $10 \mathrm{~m}$ case. 


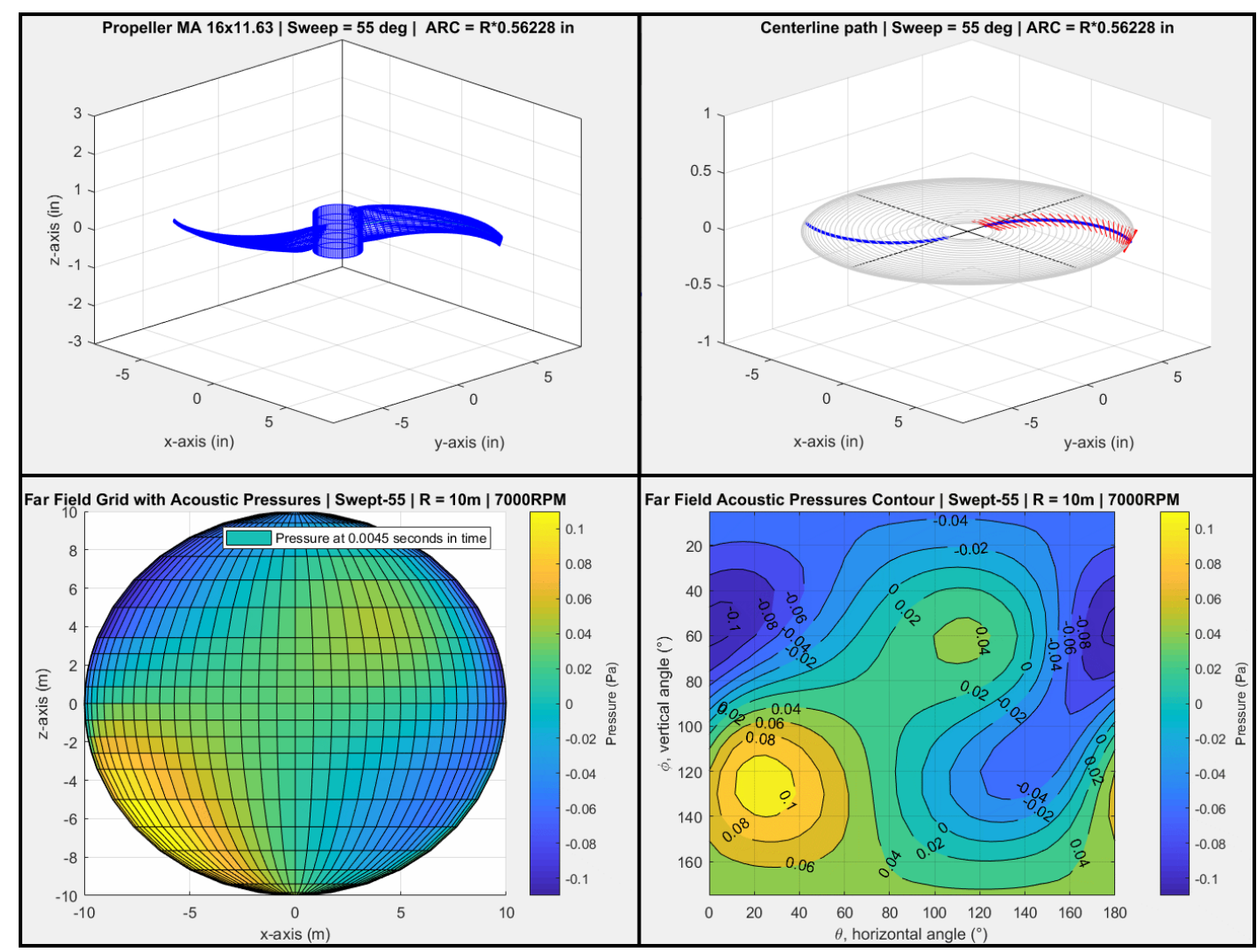

Figure 50: The swept-55 propellers geometry (top) and acoustic pressure output (below) for the 7000 RPM and $10 \mathrm{~m}$ case.

Figures 48, 49, and 50 show a consistent trend where increasing the sweep angle results in a slightly greater acoustic noise output. This is likely due to the slight increase in wetted area caused by the sweeping of the blade. The color maps show the maximum positive pressures occurring on the $\phi=60^{\circ}$ and $\phi=130^{\circ}$ axis for the largest negative and positive pressures, respectively. The large positive regions are referred to as "hot spots" and the low-pressure regions are referred to as "cold spots". In each subfigure $d$, there are two regions where large acoustic pressure "hot spots" and "cold spots" occur on the graph. The maximum and minimum pressure values are close in proximity due to the position of the propeller.

Figure 48 will be used as a reference for the multi-source method illustrated in figure 51 . The swept-35 propeller was broken into six segments from root to tip with the integration bounds shown for each segment in figure 46. The six segments were run independently through ANOPP2, with each segment producing an APTH file for each observer in the field. Afterwards, the six APTH files created for each of the observers were combined and plotted for each moment in time. Figure 51 shows the swept-35 broken into six segments in figure $45 \mathrm{~b}$, where the red line indicates the segment boundaries along the 
curved centerline. The results for the multi-source method are shown in figure 51c and 51d. They do not appear to change the graph in any meaningful way when compared to the source pair model in figure 49.

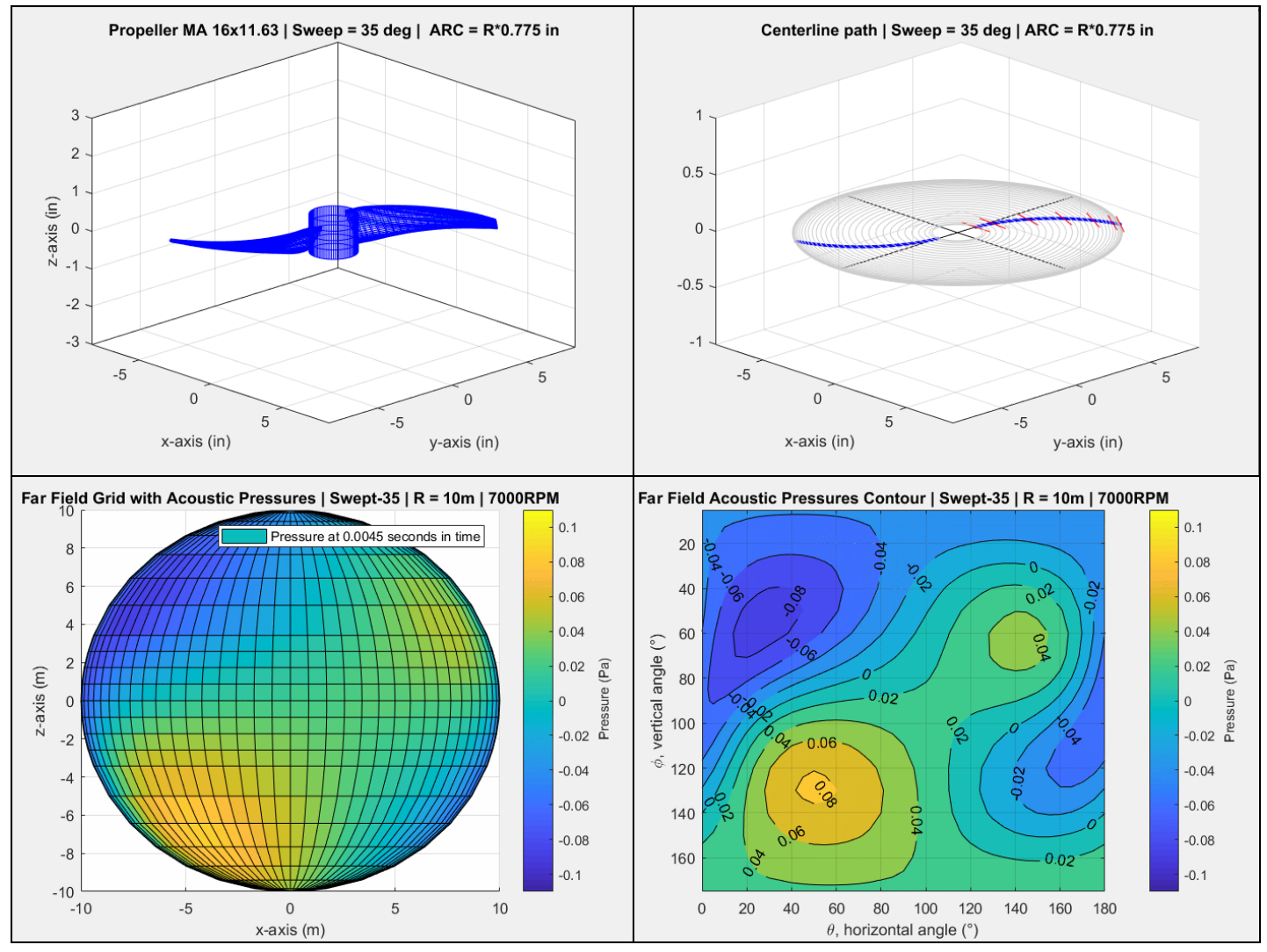

Figure 51: The swept-35 propeller split in multiple source points across each blade.

The previous analyses only looked at one semi-sphere observer distance and one RPM value. ANOPP2 allows us to perform simulations over a wide range of distances and RPMs for the propeller shapes under consideration, which may provide more insights into the relationship between RPM, distance, and observer angle for each of the propeller designs. It is well established that higher RPM (i.e. tip speed) increases noise while larger distances reduce the noise. However, the trends may vary for each blade. To test this, three plots have been made to show how the below-plane, in-plane, and above-plane of rotation pressures vary as a function of RPM and distance. Each plot uses a single observer along the $\theta=$ $90^{\circ}$ line to evaluate the trends, with $\phi=50^{\circ}$ as our above-plane observer point, $\phi=90^{\circ}$ as the in-plane of rotation observer point, and $\phi=120^{\circ}$ as the below plane of rotation angle. Recording the acoustic pressures for all RPMs, angles, and distances for each propeller design provides clear trends regarding the aeroacoustic effect of adding sweep to a propeller. The APTH file for each condition was compared 
on a single plot using equation (37) which calculates $p_{r m s}$. The pressure values from each APTH file are used to compute a root mean square to produce single pressure that represents the average pressure experienced by the observer. This value is then converted to $\mathrm{dB}$ with the reference pressure set to $20 \times 10^{-6} \mathrm{~Pa}$ in equation (38).

$$
\begin{aligned}
& P_{r m s}=\sqrt{\frac{{p^{\prime}}_{1}^{2}+{p^{\prime}}_{2}{ }^{2}+p^{\prime 2}{ }_{3}+{p^{\prime}}^{2}+\cdots+p^{\prime 2}}{\text { number of pressure values }}} \\
& d B=20 * \log _{10}\left(\frac{\text { Prms }}{\text { Pref }}\right)=20 \log \left(\frac{\text { Prms }}{20 * 10^{-6}}\right)
\end{aligned}
$$

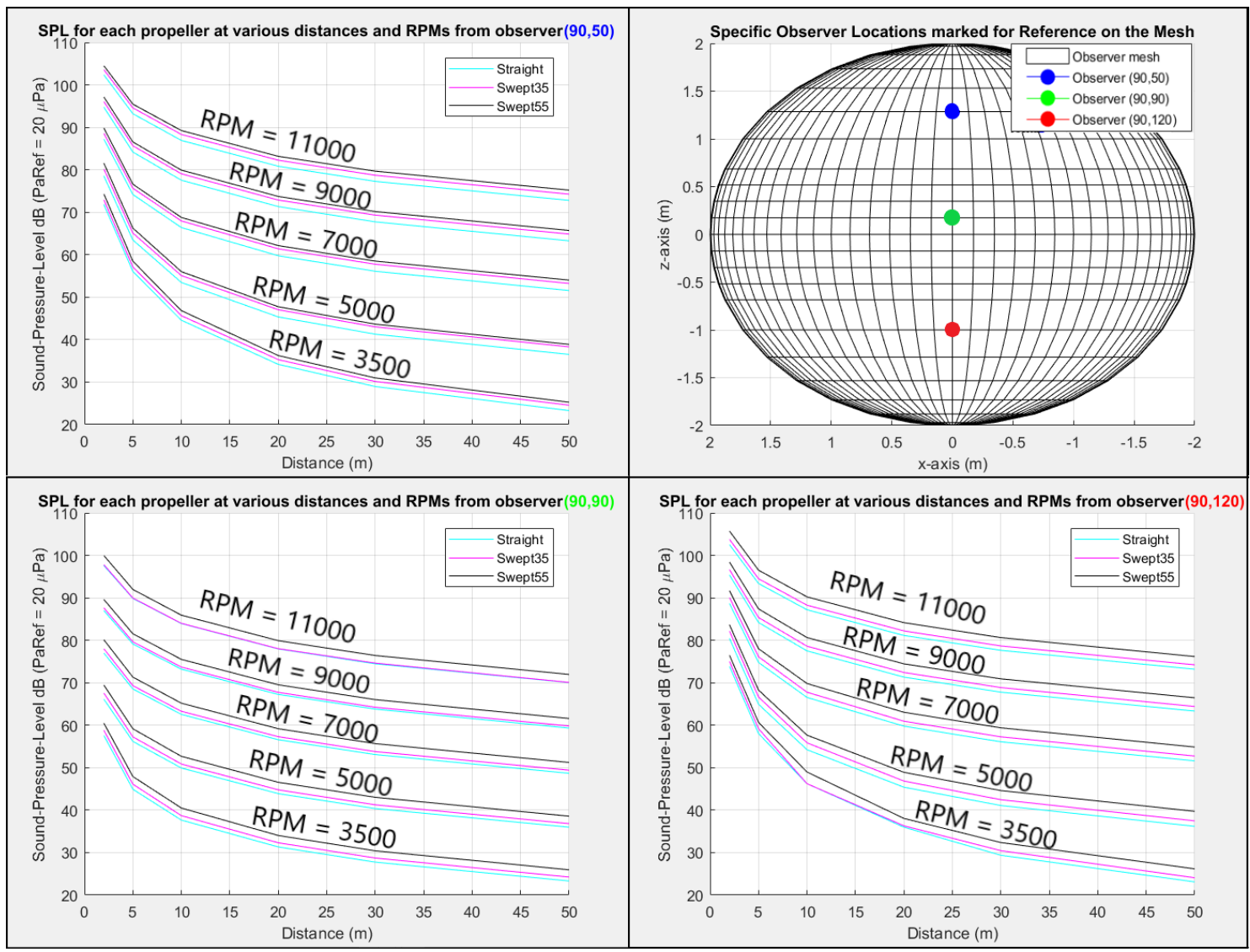

Figure 52: General trends for three different propellers in ANOPP2 at various RPM's and distances for three observer positions.

The trends are consistent across all obsevation points. The straight propeller is slightly quieter than either of the swept designs. The trends are consistent with far-field data where the straight propeller's contour plots would produce slighly less intense acoustic pressures for a given distance and RPM. The increase in acoustic pressures is likey due to the increasing arc length for larger swept propellers. Since the F1A intergral is performed over a larger arc length, the result is a greater pressure disturbance. But 
this fact alone does not explain why the swept propellers are producing more noise over the straight design. A comparison between the various far-field pressure plots provides additional insight. Figure 53, 54 , and 55 will examine each propeller at a fixed distance, $50 \mathrm{~m}$, from the lowest-to-highest RPM to study how the far-field pressure contour varies.

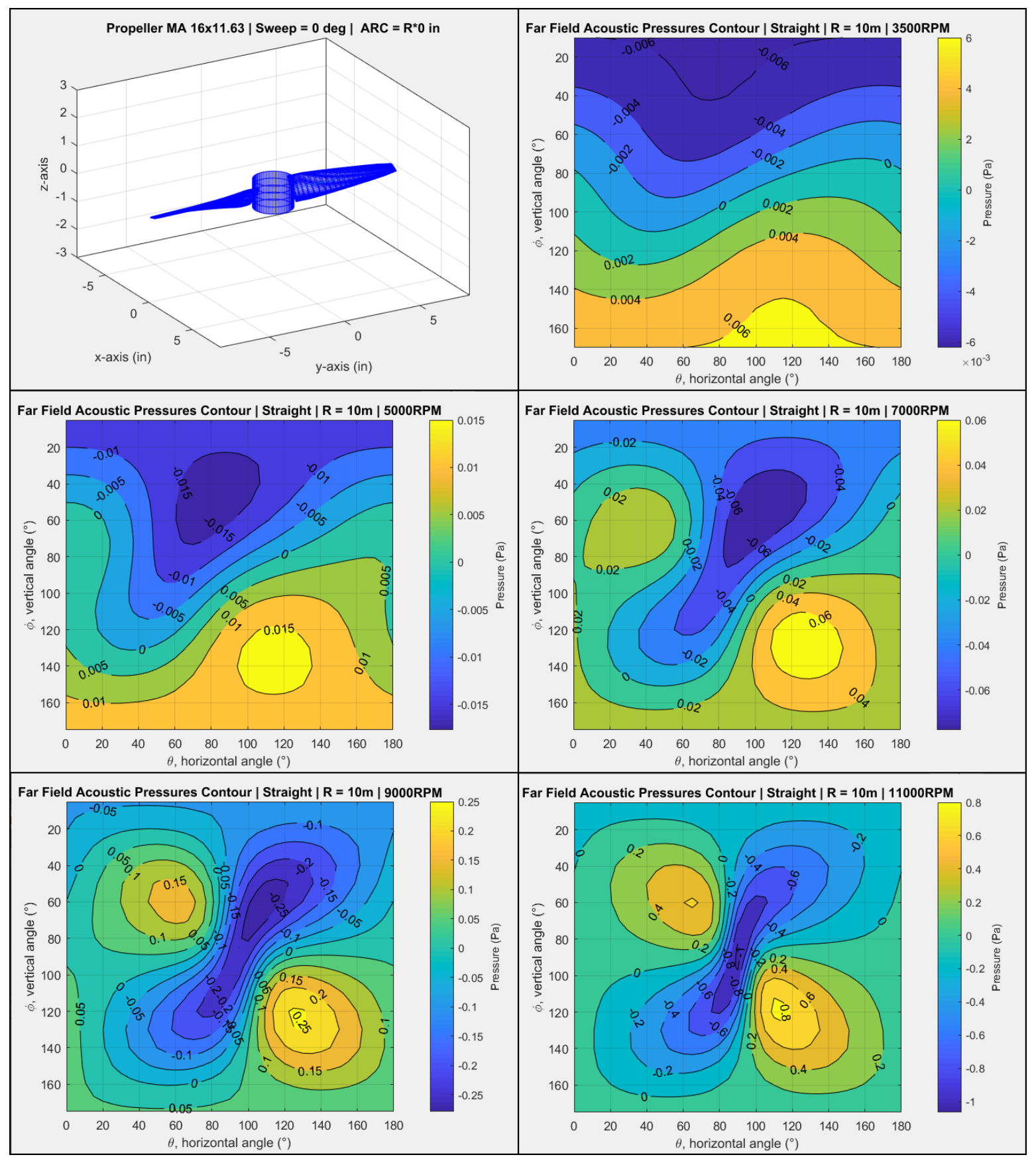

Figure 53: The straight propeller's acoustic pressure at various RPMs recorded at $10 \mathrm{~m}$ from all angles on the SemiSphere. 


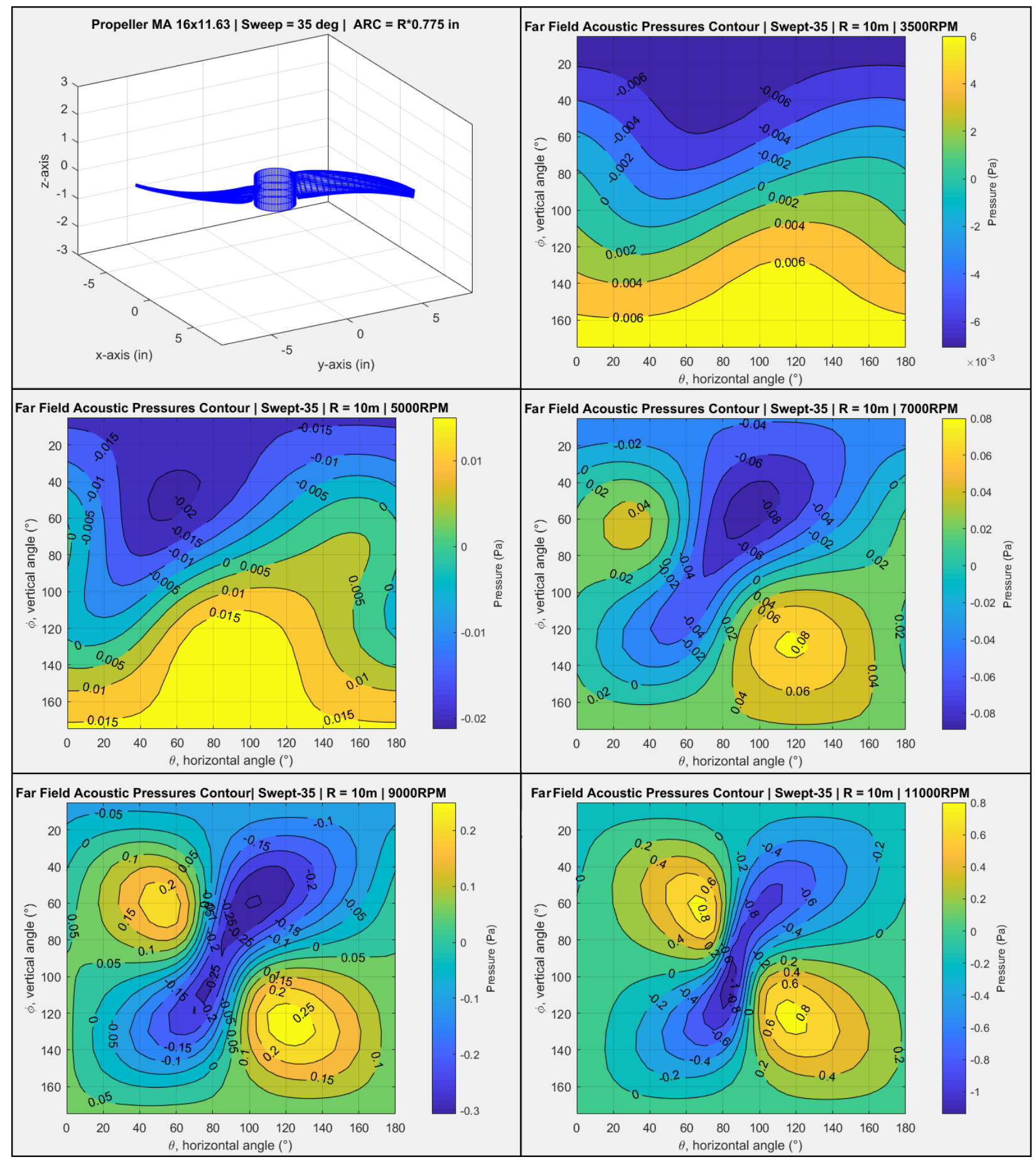

Figure 54: The swept-35 propeller's acoustic pressure at various RPM recorded at 10m from all angles on the Semisphere. 


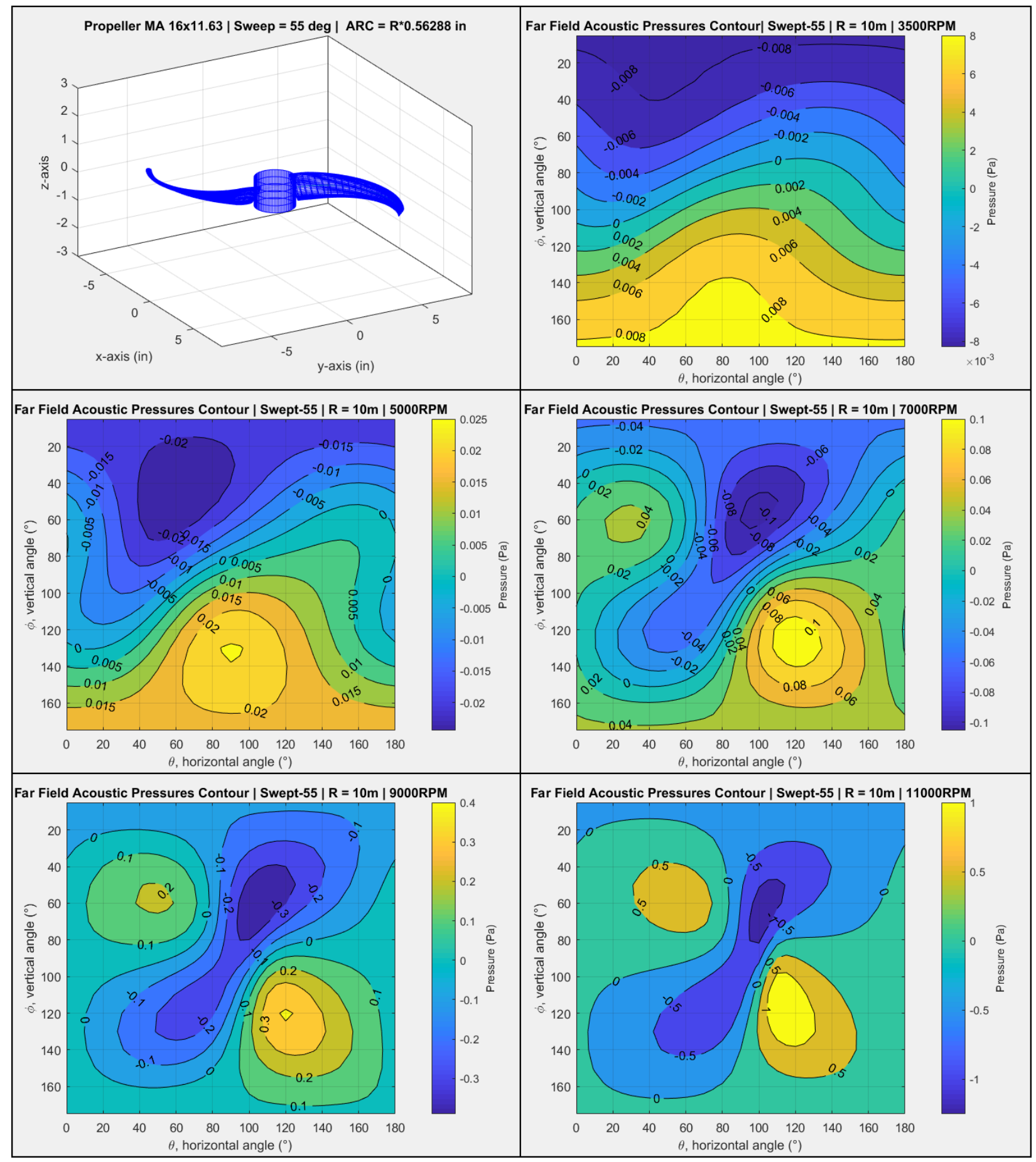

Figure 55: The swept-55 propeller's acoustic pressure at various RPM recorded at 10m from all angles on the Semisphere. 
At 3,500 RPM the contour starts off with weak pressure trends where the largest positive and negative acoustic pressures occur near the poles with the in-plane region producing minimal pressures. There are slight "bumps" in the far field caused by the propeller's radial position. At 5,000 RPMs, the "bumps" start to detach from the poles and approach the plane of rotation, $\phi=90^{\circ}$, while producing progressively larger acoustic pressures. This trend continues with RPMs over 7,000 but a second pair of weaker pressure regions start to develop behind the initial pair. This is likely a result of the loading (moving dipole) sources, which increase in strength as the forces, (lift and drag) increase with higher and higher rotational speeds. However, this does not explain why the far field produces the complex contour shape seen at higher RPMs. Breaking the total acoustic pressure contour plot into its component sources, should shed additional light onto the phenomenon behind the pressure contours in figures 53 , 54 , and 55.

Figures 56, 57, and 58 separate the total acoustic pressures into their individual source components. Which is the loading (moving dipole) and thickness noise (moving monopole) for various RPMs. Figures 56,57 , and 58 are arranged so that the left subfigures with "spring colors" are the results of the loading noise and the middle subfigures in "winter colors" are the thickness noise and the subfigures on the right represent the summation of the pressure components. This is the total noise. For each propeller, the loading noise at 3,500 RPM produces a weak two-set dipole shape in the far field with the largest positive-negative pressure values occurring at poles for the observer grids. This explains the "bump" seen in previous figures with RPMs at 3,500. As RPM increases the sources become stronger, resulting in sharper gradients and larger acoustic pressures in the far field as seen in the 11,000 RPM subfigures. An interesting note about the 11,000 RPM thickness noise is the "spreading" of the loading noise across the observer field. The sharp and sudden dip in pressures seen in the thickness noise plots vary significantly across blade shape. This implies one of two major points. One, sweeping the propeller blade allows for a more gradual thickness noise to be introduced to the observer since the airfoils are no longer "stacked" when they pass the observer. This results in a spreading and overall decrease in noise generated by the thickness of the blades. Two, the thickness and loading APTH files can be altered as a result of sweeping the blade. This means, that there is a potential case cause deconstructive interference between the radiated pressures. The interference between the current propeller designs is plotted in figure 59. 


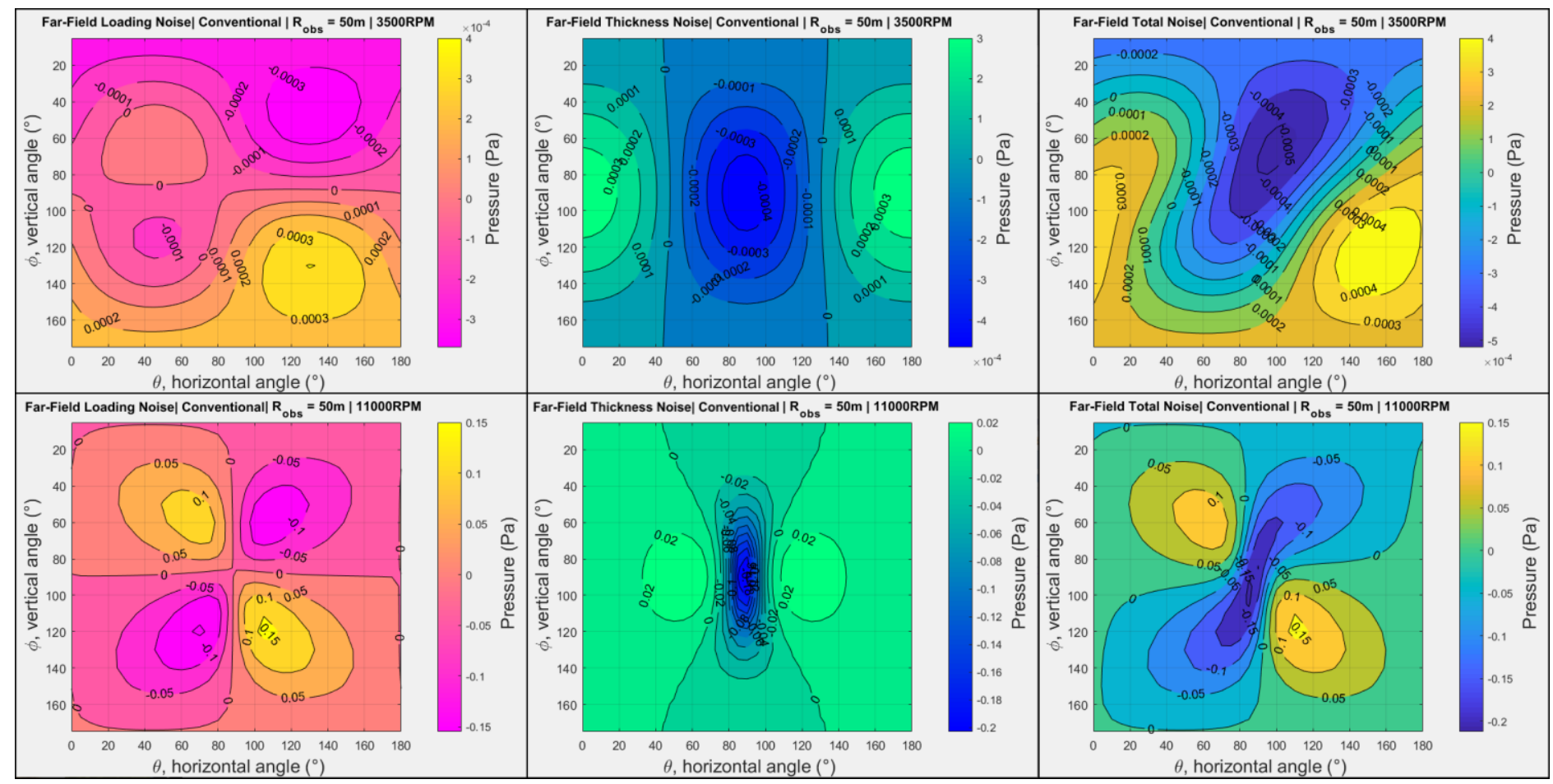

Figure 56: Dipole and monopole contribution to the total pressure for the straight 2-blade propeller at $50 \mathrm{~m}$.

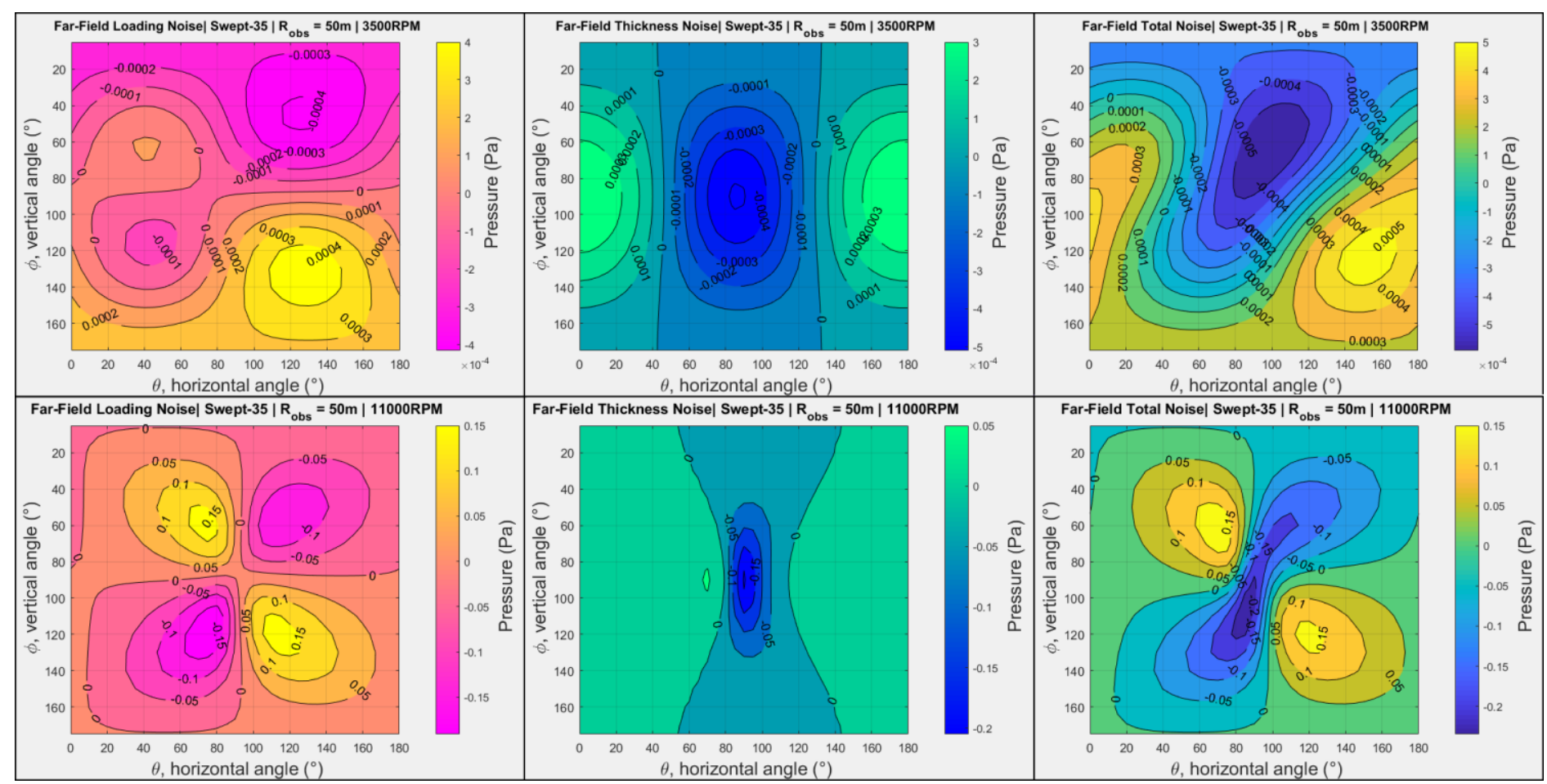

Figure 57: Dipole and monopole contribution to the total pressure for the swept-35, 2-blade propeller at $50 \mathrm{~m}$. 


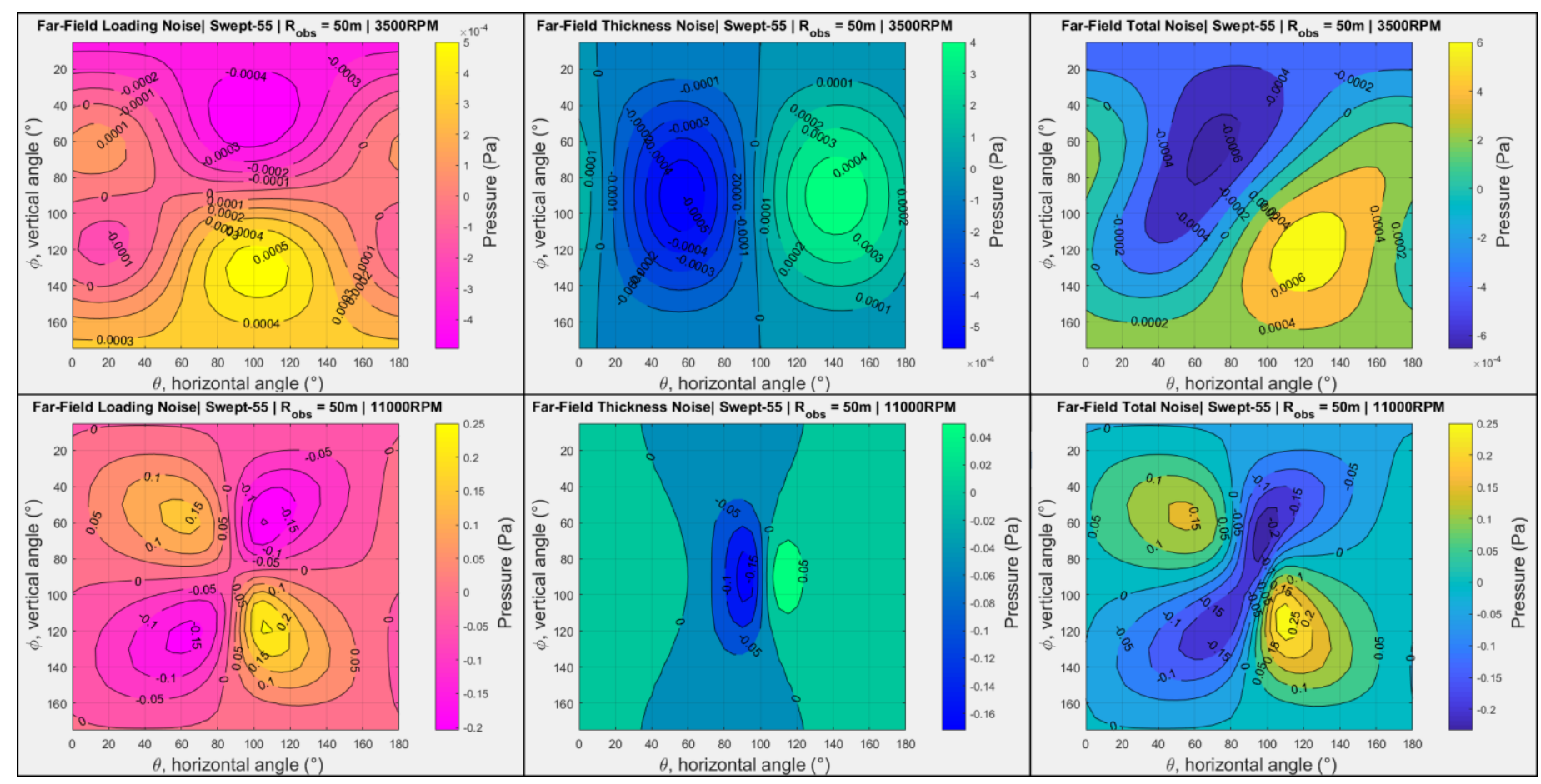

Figure 58: Dipole and monopole contribution to the total pressure for the swept-55, 2-blade propeller at $50 \mathrm{~m}$.

Figure 59 sheds further light on the thickness and loading noise contributions. A single observer was selected from the plane of rotation $(90,90)$ to compare each propeller type at a constant speed of 11,000RPM and 50meters. These parameters provide a clear visual difference in pressure history trends. The individual source contributions are plotted with respect to the total noise. The thickness noise, marked with red dash-dot lines, produced a large, sudden drop in pressure as the blade "passes" the observer. Since the straight, conventional propeller has the entire blade "pass" the observer at once, the negative pressure should be larger than the swept designs. The swept designs produce a gradual negative pressure drop over a longer duration of time since the observer only "sees" portions of the blade pass at any given moment. The loading noise, marked with black dotted lines, shows a gradual sinusoidal wave. This seems reasonable as the blade is producing a constant load so the pressure signature will grow and shrink as the blade rotates toward and away from the observer. These individual sources, along with their phase, culminate into one total wave, marked in blue.

Comparing the straight propeller to the swept-35 design, there is little difference in magnitude or any apparent phase cancelation. There is a minor difference in the thickness noise at the peaks and a slightly smaller drop in thickness noise for the swept-35, but the trends are otherwise identical in waveform. 
The swept-55, however, shows a stark contrast to the straight propeller. The thickness magnitude is noticeably smaller and extends over a longer duration of time. Another fascinating aspect of the swept55 is the loading noise, which differs in shape and magnitude. The swept-55 has a loading noise with larger peaks and smaller troughs with respect to the previous designs. However, the loading and thickness noise create positive interference during the approaching portion of the revolution. This created a large, positive pressure spike that did not occur in previous designs. This suggests that the swept designs are noisier than the conventional design, even when the thickness noise is reduced. The propellers under current evaluation are not optimized for interference; rather, they serve as a validation method for the model. If the experimental results match the F1A prediction, then an optimization method could be applied to find a sweep angle that produces maximum destructive interference. This analysis extensively covers the 2-bladed design but has not discussed, in any significant detail, the noise characteristic of larger blade counts. Higher blade counts could contribute to a different waveform, especially if the blade count is high enough and the blades are swept significantly enough to cause overlap between the tip of one blade and the root of the following blade, as in the case of the swept-55 propeller with a blade count of 4 . Figure 29 show the swept-55 propeller has blades that slightly overlap between the tip of the proceeding blade and the root of the preceding blade. However, additional blade counts increase the simulation time considerably. The simulation time could be justified if the introduction of additional blades altered the pressure waveform in a significant way. A simulation was constructed to compare the two, three, and four bladed propellers blade counts for a straight and swept-55 propeller. An observer was placed at the $(90,90)$ in-plane position at 50m. All propellers were operated at an RPM of 5000 . Figure 60 shows the result of adjusting the blade count for the two, three, and four blades, respectively. The trends show that a similar waveform appears across all blade counts. It is important to note that the acoustic pressure slightly decreases with the increasing blade count. Otherwise, the waveform is only repeating more frequently as a result of more blades passing for the same duration of time. This suggests that the two-bladed propeller will suffice for the analysis and higher blade counts will see similar trends as the two-bladed design. 

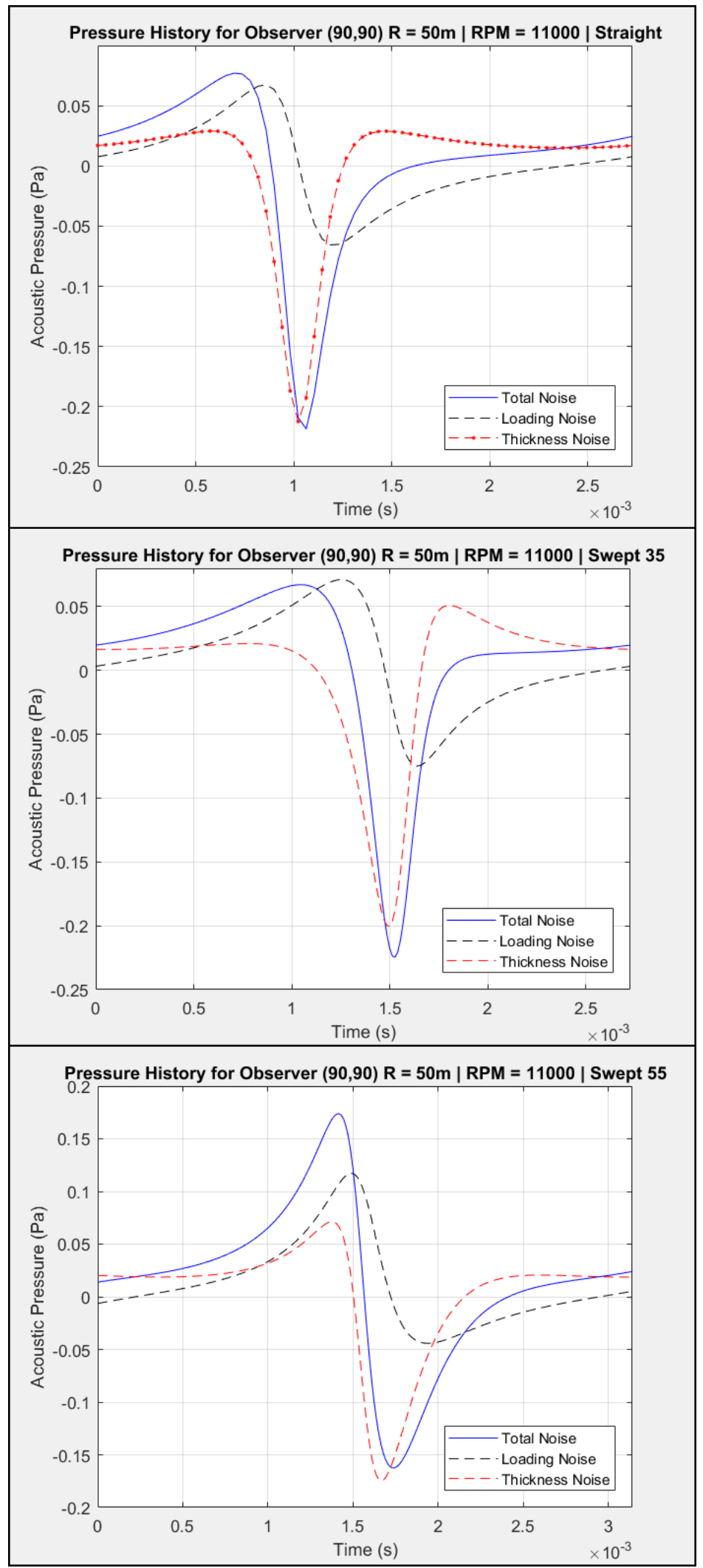

Figure 59: Pressure history components for each propeller at observer $(90,90)$. 

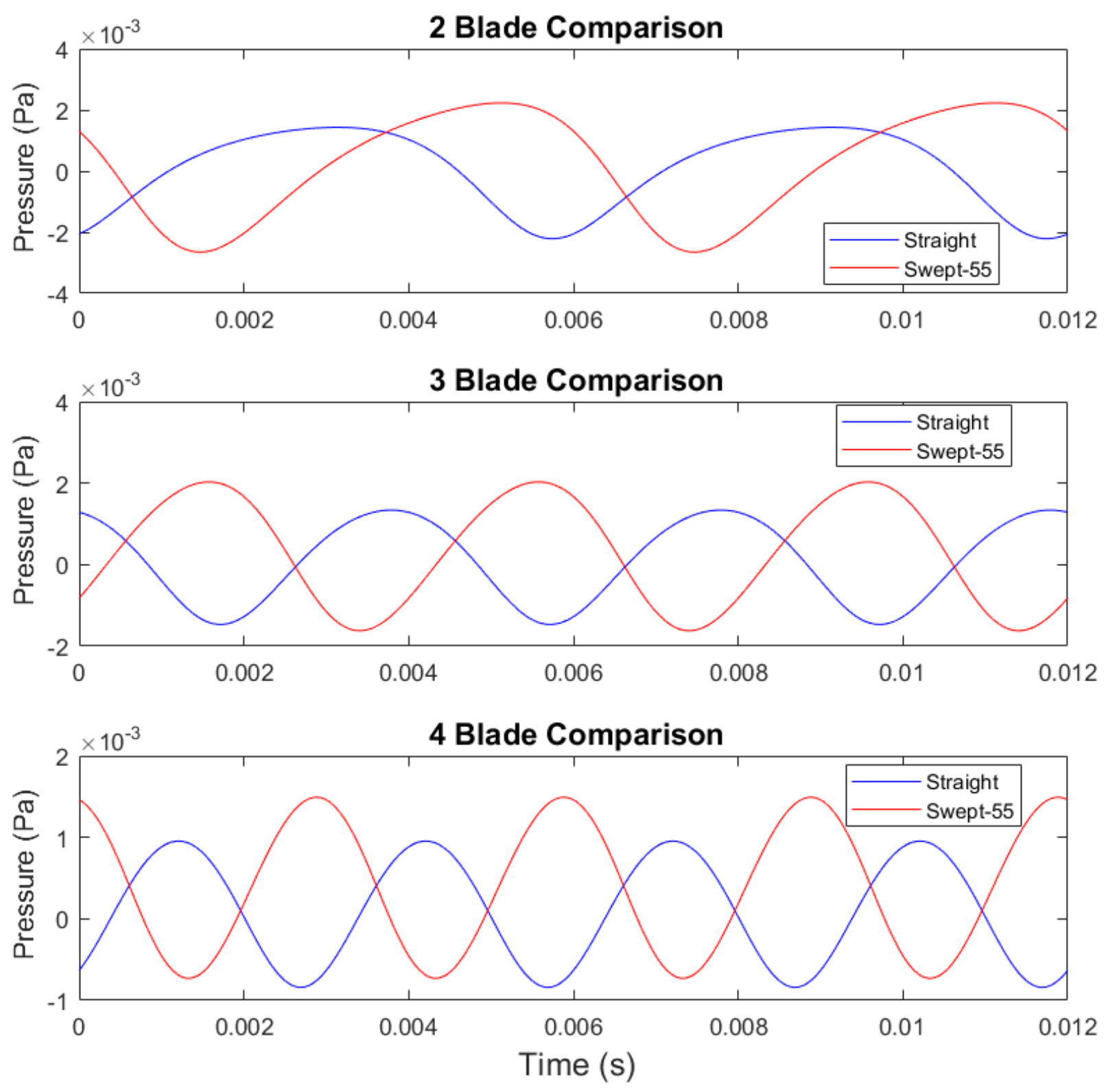

Figure 60: Straight and Swept-55 compared across various blade counts from observer $(90,90), 50 \mathrm{~m}$ away and operating at an RPM of 5000.

\subsection{AERODYNAMIC PERFORMANCE}

Each propeller was designed to be tested for a 2-blade, 3-blade, and 4-blade configuration. The propeller designs are represented in table 10 which shows the various blade configuration and designs intended for aerodynamic evaluation. The X's indicate which testing conditions were successful. Not all conditions could be evaluated due to the variance in weight/mass distribution across the propeller blades 
that were manufactured with the 4DOF method. The propellers manufactured with the 4DOF method did not produce consistent mass distributions. This caused the center of mass to shift away from the center of rotation. This led to excessive vibrations at low RPMs, preventing collection of any aerodynamic data. The only propellers that were milled with this method were the four swept-15 propellers and two of the straight propellers. And of these propellers milled with this technique it was still possible to obtain a few balanced configurations with light sanding. The straight designs could be tested for the two and three blade configurations while the swept-15 could only provide a balanced 2-blade configuration.

\begin{tabular}{|l|l|l|l|}
\hline & 2-blade & 3-blade & 4-blade \\
\hline Straight & X & X & \\
\hline Swept-15 & X & & \\
\hline Swept-35 & X & X & X \\
\hline Swept-55 & X & X & X \\
\hline
\end{tabular}

Table 10: $X==$ successful evaluation in the wind tunnel at 3000 RPM

\subsubsection{TESTING THE TWO BLADED PROPELLERS}

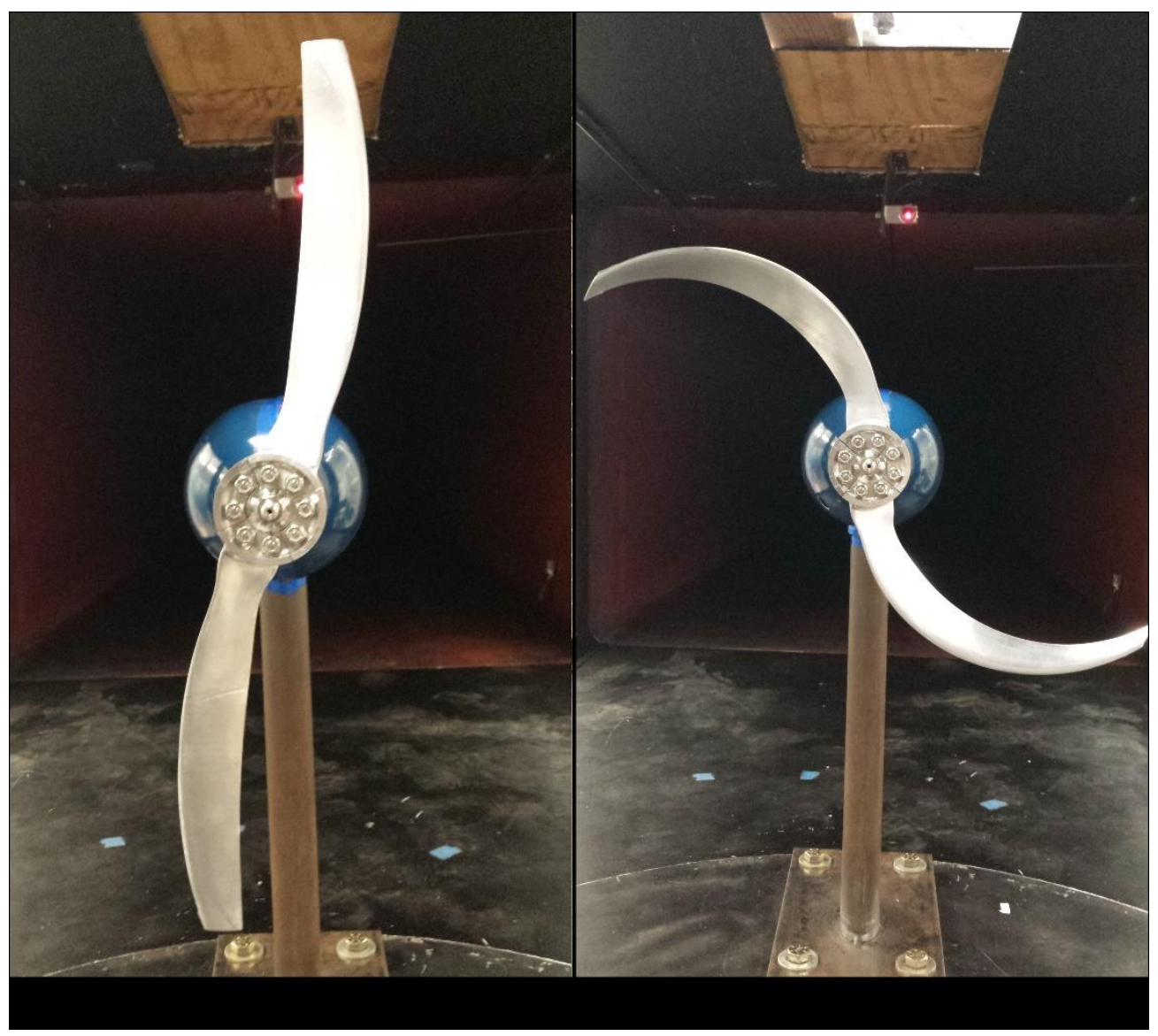

Figure 61: Swept-15 (left) and swept-55 (right) inside ODU's wind tunnel. 


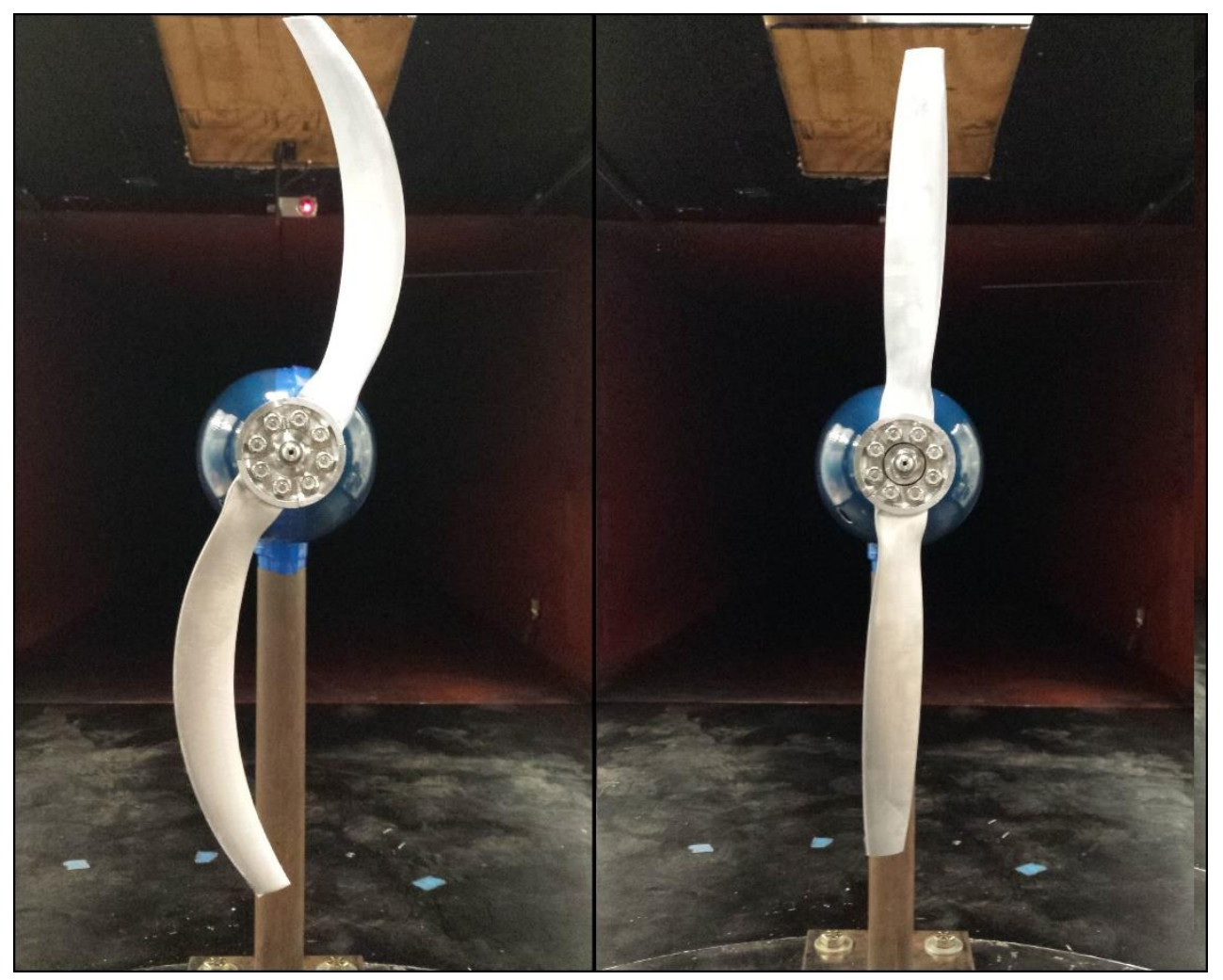

Figure 62: Swept-35 (left) and straight (right) inside ODU's wind tunnel.

The first propeller configuration tested was the 2-blade design at 3,000 RPM. All propellers designs had a balanced set that produced minimum vibrations. All 2-bladed designs were tested with hub plates which can be seen in figures $61 a, 61 b, 62 a$ and $62 b$ which were secured to the hub mount with shoulder bolts. The aerodynamic values of interest are coefficient of thrust $(\mathrm{Ct})$, coefficient of power $(\mathrm{Cp})$, and efficiency (n). The Ct plot, figure 63, shows significant overlap of trends between the swept-35, swept-15, and the straight propeller across the entire range of advance ratios. The $95 \%$ Confidence Intervals $(95 \% \mathrm{Cl})$, signified by the dotted lines, show that the coefficient of thrust is consistent across the first three blade designs. The only propeller designs that deviates from this consistency is the swept-55 and even with this dramatic sweep, the coefficient of thrust only shifts down slightly for the lower range of advance ratios. This would suggest that the swept-55 is a disadvantageous design, but the coefficient of power plot, figure 64 , shows the swept-55 requires less power to operate the same RPM.

The coefficient of power plot mirrors the trends seen in Ct. That is, major overlaps occur for the swept-35, swept-15, and straight propellers. The swept-35 requires a slightly larger input power compared to the straight and swept-15 propellers, which have the same thrust outputs. However, these variations in thrust 
and power are ultimately canceled out in the efficiency plot, figure 65. This figure shows that the deviation found in the previous two graphs ultimately cancel out in efficiency. All four propeller blade types overlap across all the advance ratios below $\mathrm{J}=0.6$. At advance ratios beyond peak efficiency, small deviations in smoothness and imperfections are likely the main factors in the trend variations above $J=0.6$. This would explain why the swept-35, which did not suffer from any manufacturing defects, performs better than the other designs after peak efficiency.

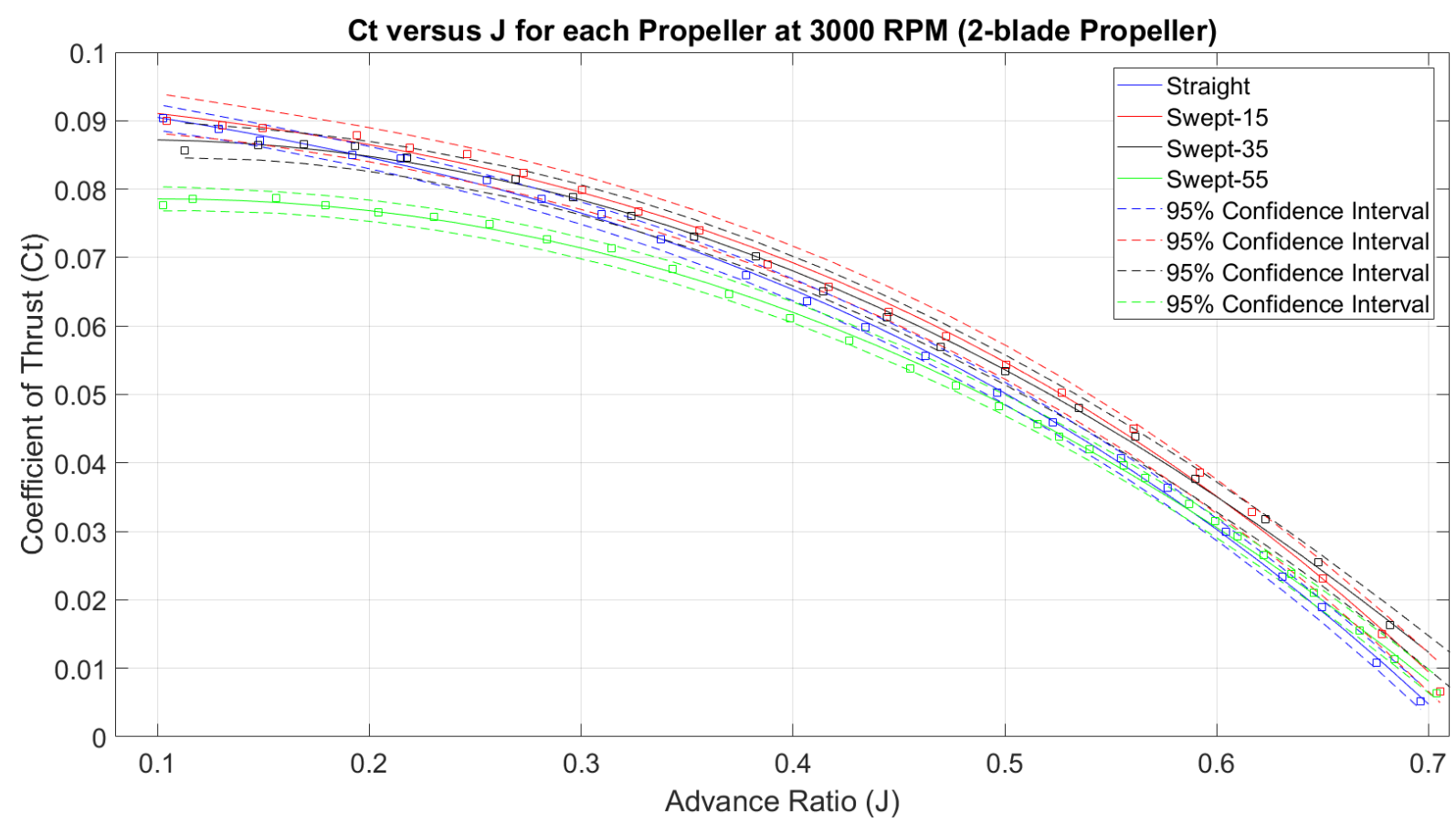

Figure 63: Coefficient of power for all 2-bladed propellers. 


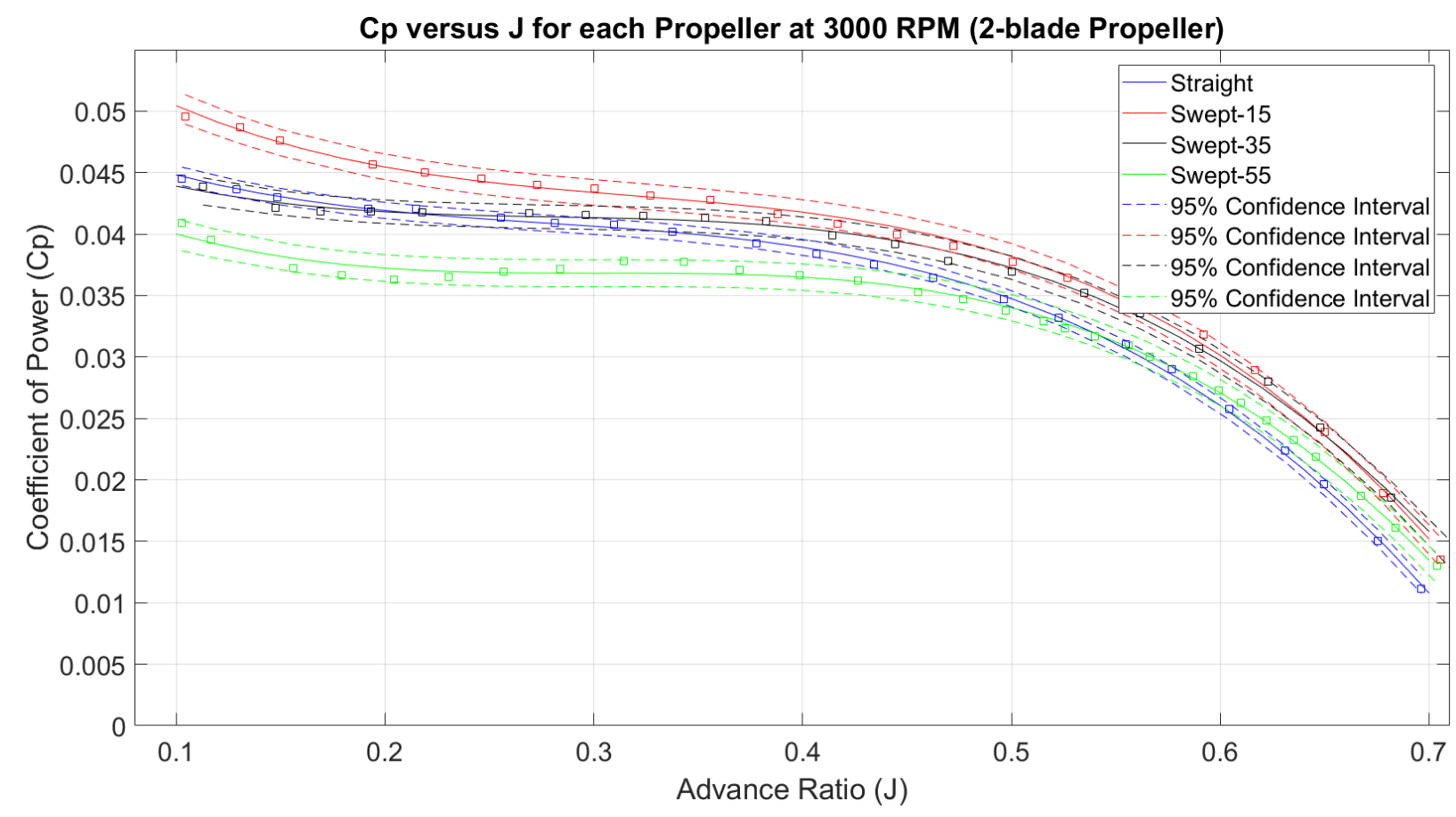

Figure 64: Coefficient of Power for all 2-bladed propellers.

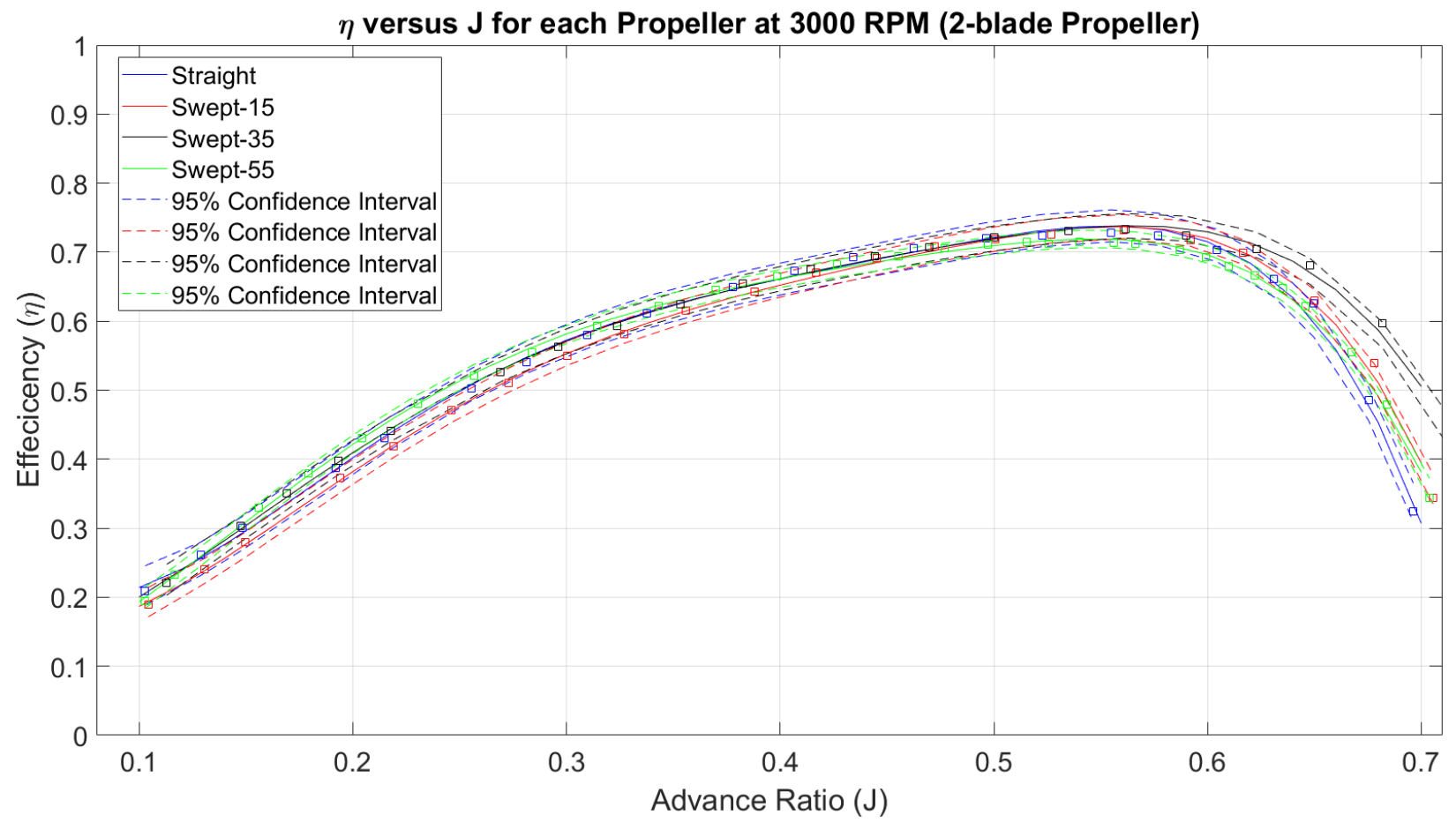

Figure 65: Efficiency for all 2-bladed propellers. 


\subsubsection{TESTING THE THREE BLADED PROPELLERS}

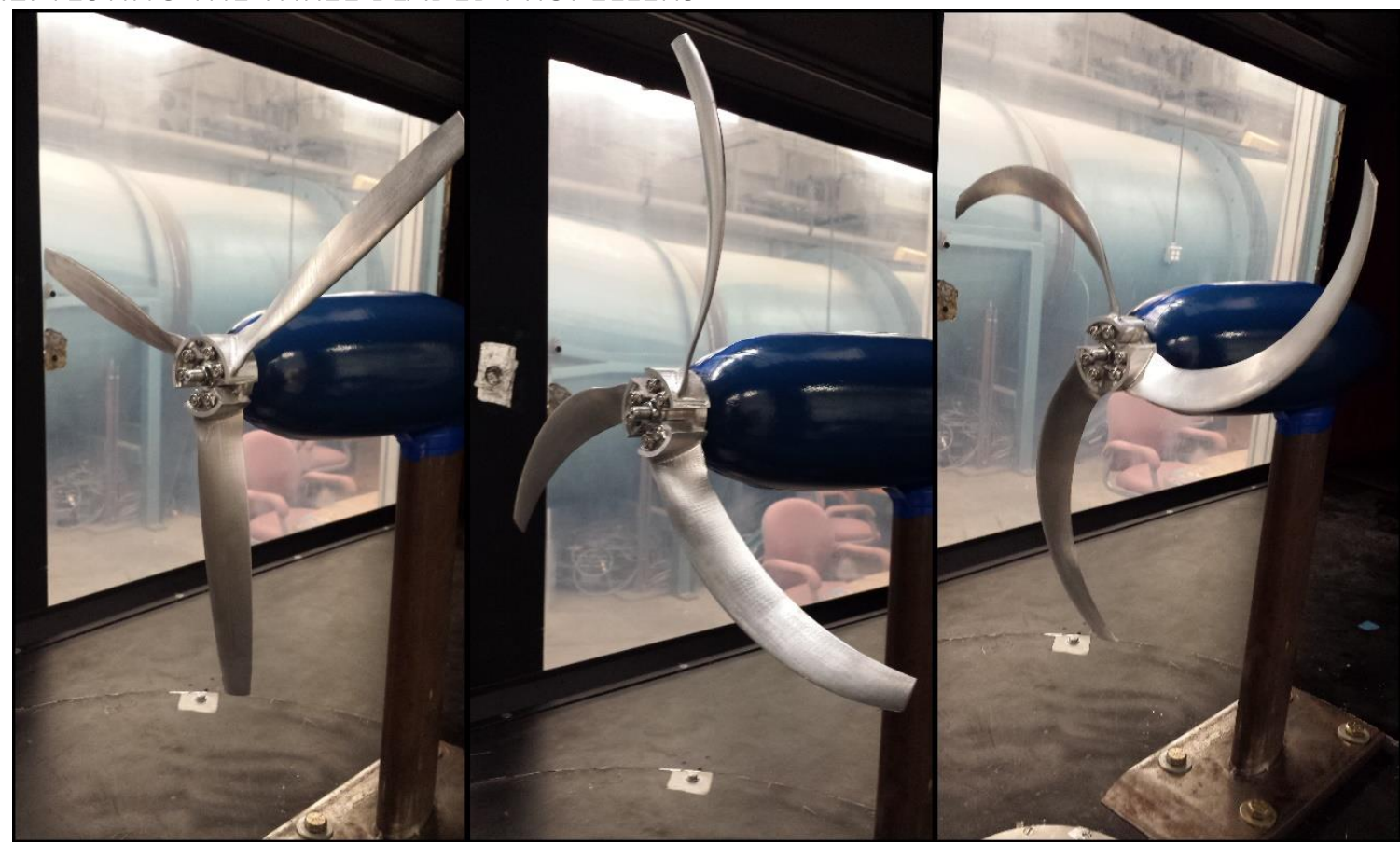

Figure 66: The three different designs tested in ODU's wind tunnel for the three-blade configuration.

Of the four propeller designs, only three could be evaluated for the three-blade count. As stated, this was due to deviations in production for the 4DOF milling method. The successful designs can be viewed in figure $66 a, 66 b$, and $66 \mathrm{c}$ which shows the straight, swept-35, and swept-55 respectively. Theses propellers were tested at 3000 RPM and produced similar results for the efficiencies in figure 69. The trends overlap until peak efficiency is reached, afterwards the trends start to diverge. The other interesting feature to note is the swept-55's juxtaposition in magnitude with respect to the other propellers. The swept-55 now has a greater $C_{t}$ and $C_{p}$ than the other two configurations, while maintaining congruent efficiencies. 


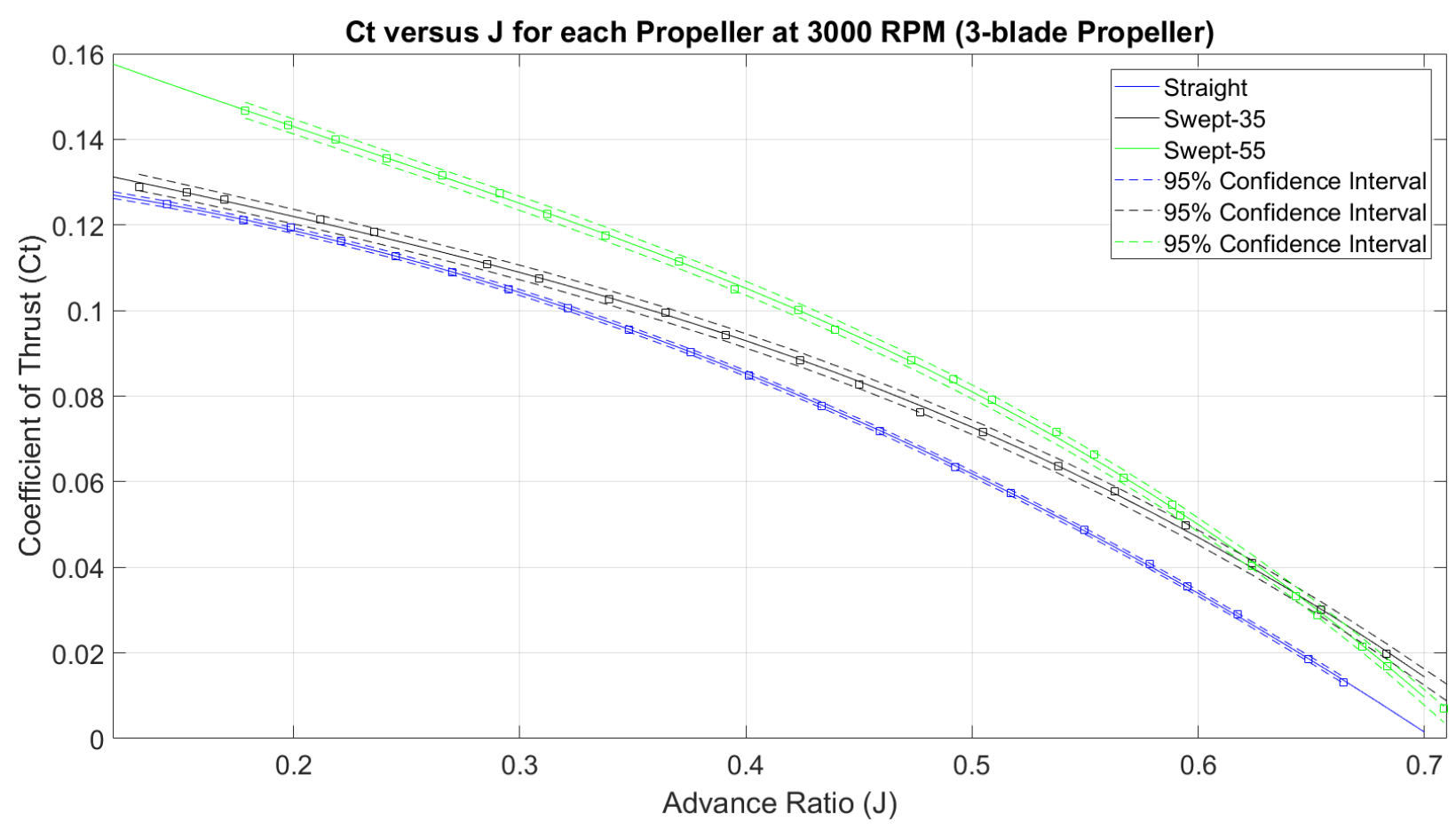

Figure 67: Coefficient of thrust for the straight, swept-35, and swept-55 3-bladed propellers.

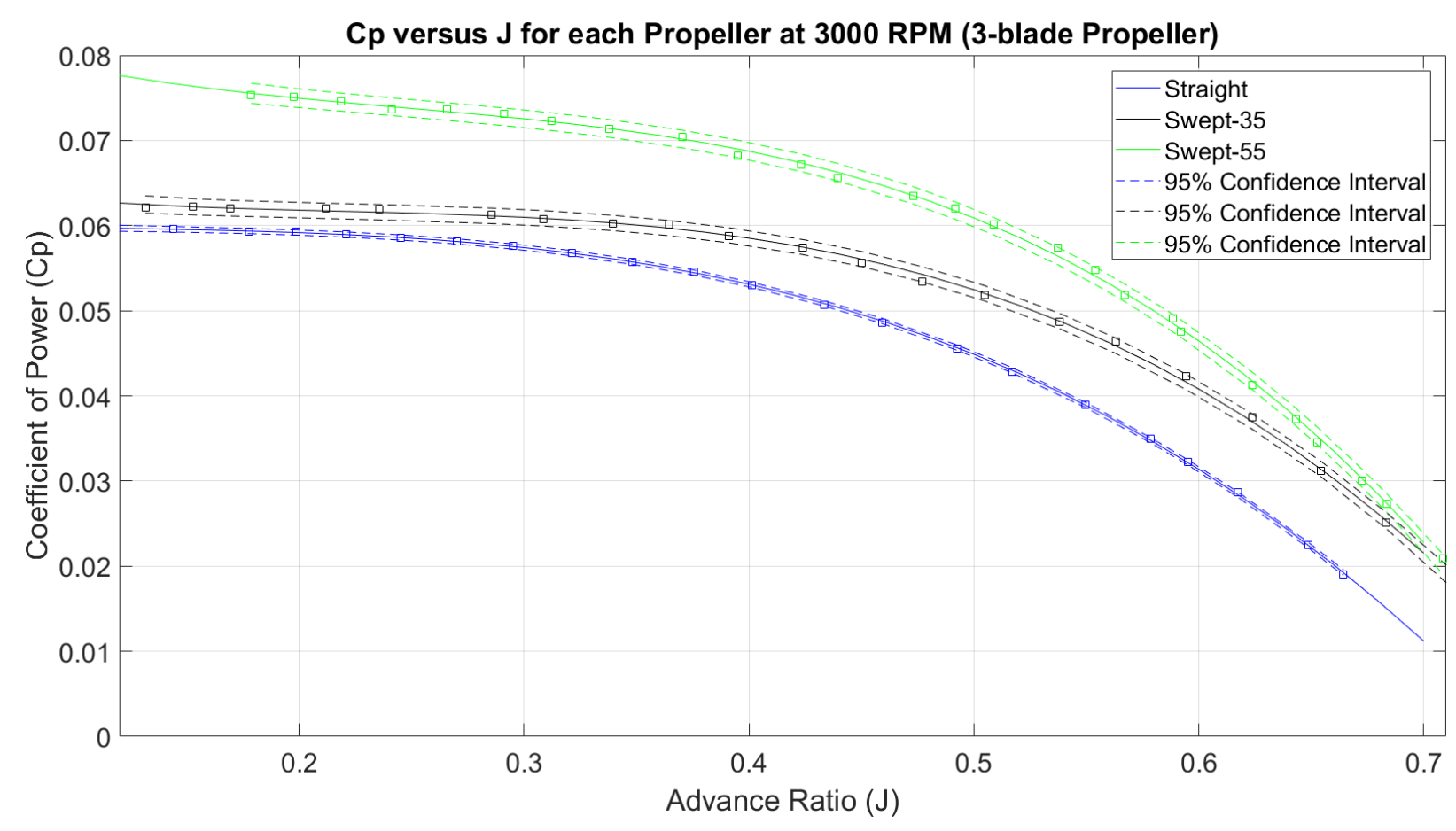

Figure 68: Coefficient of power for the straight, swept-35, and swept-55 3-bladed propellers. 


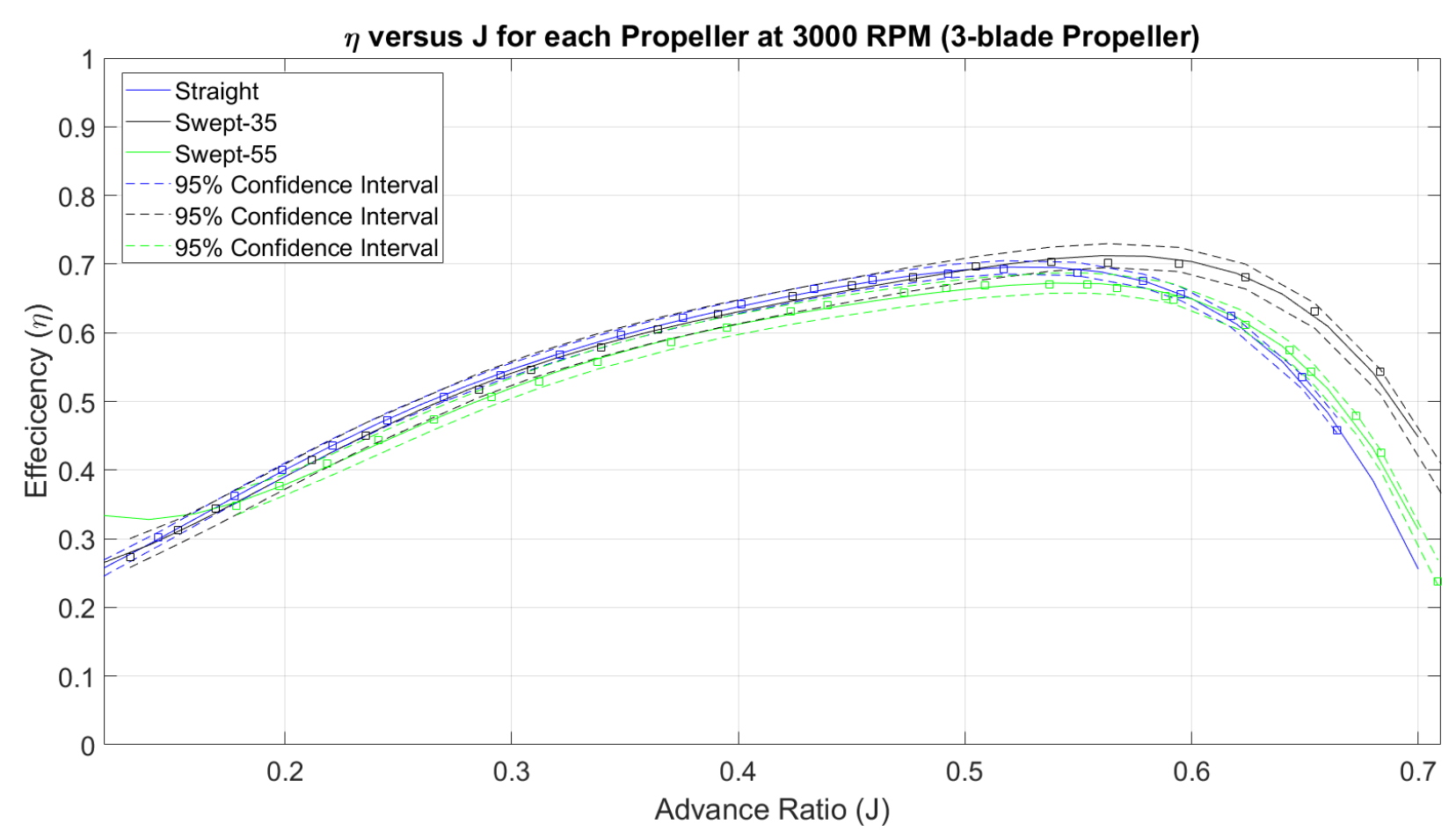

Figure 69: Efficiency for the straight, swept-35, and swept-55 3-bladed propeller.

\subsubsection{TESTING THE FOUR BLADED PROPELLERS}

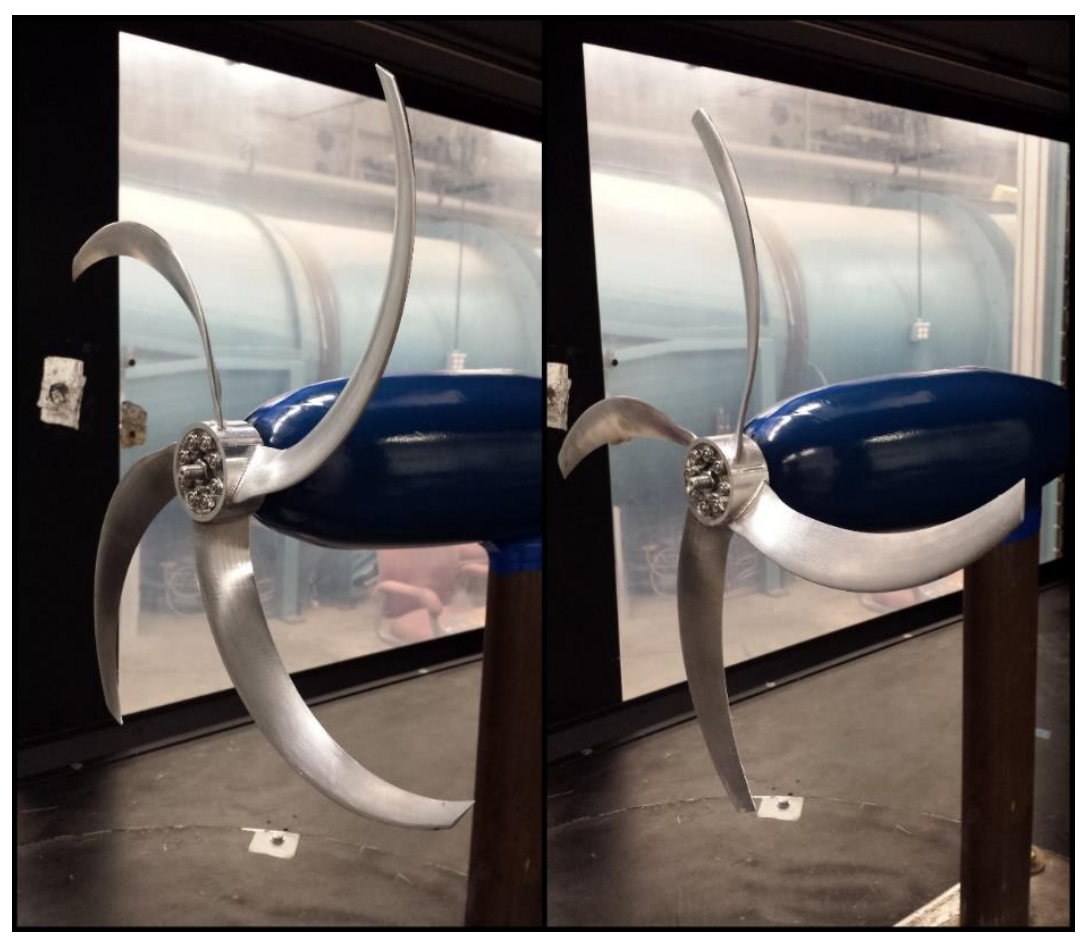

Figure 70: The two different designs tested in ODU's wind tunnel for the four-blade configuration. 
Only two designs, the swept-55 and the swept-35 could be balanced for evaluation with the 4blade configuration. Again, figures 71, 72, and 73 show consistent trends with respect to the available data from the lower blade number experiments. The consistency in which the propellers efficiencies stack across all blade numbers show that these trends are robust. Even with the missing data, it is reasonable to conclude that, for the propellers evaluated, blade sweep does not produce any significant deviation in the efficiency in comparison to a conventional, straight propeller. Additional testing should be performed on propellers of various diameters and twists before a general conclusion can be made about the sweeps impact on the aerodynamic efficiencies, but this test provides solid groundwork for future designs. With consistent efficiencies established, the acoustic waveform needs to be examined. This will determine if the ANOPP2 lifting line model is sufficient for predicting the noise and determine if the ARC swept propellers produce less noise. The next experiment will investigate the propeller noise across the various blade designs.

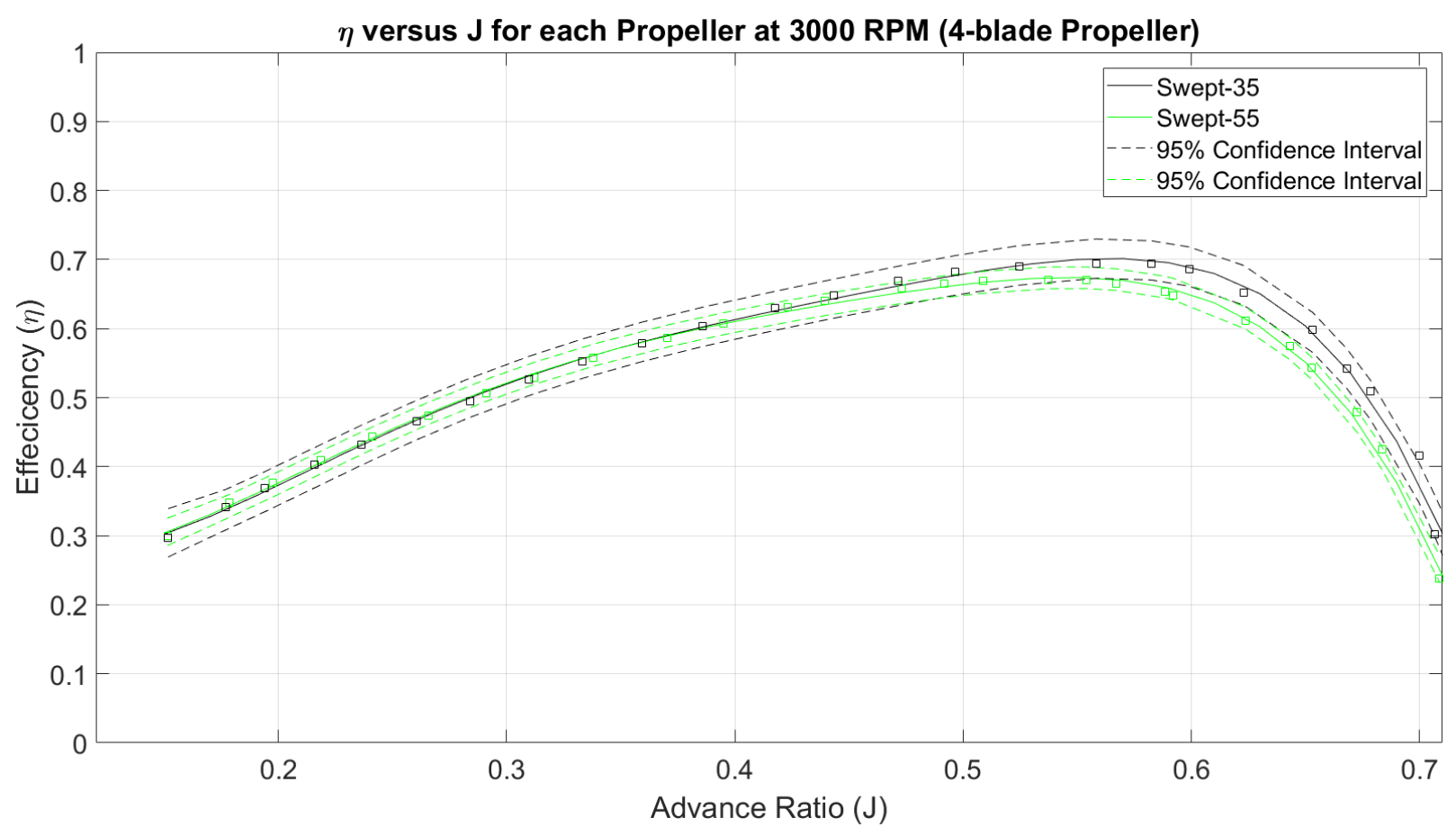

Figure 71: Efficiency for the swept-35 and swept-55 4-bladed propellers. 


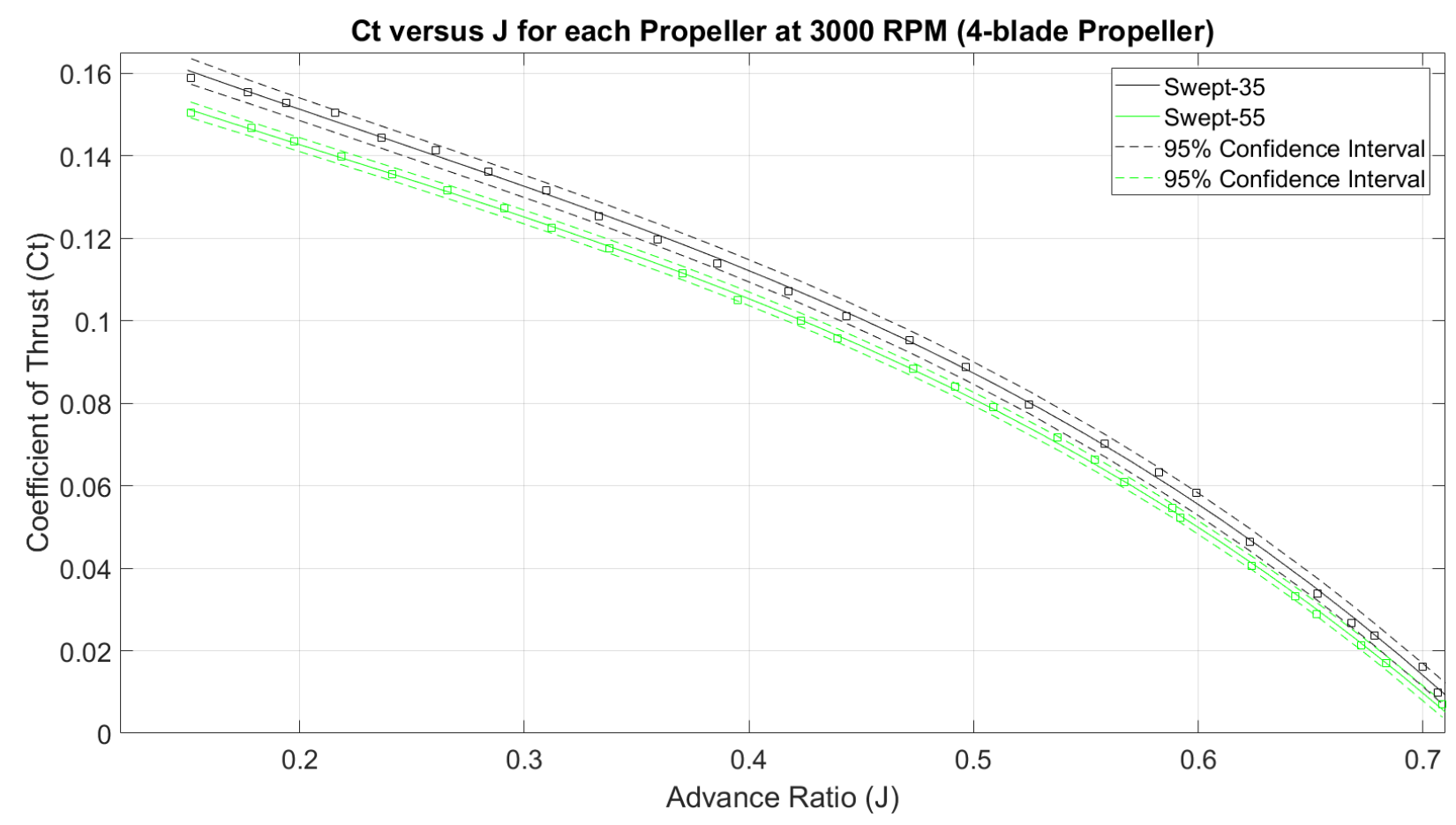

Figure 72: Coefficient of thrust for the swept-35 and swept-55 4-bladed propellers.

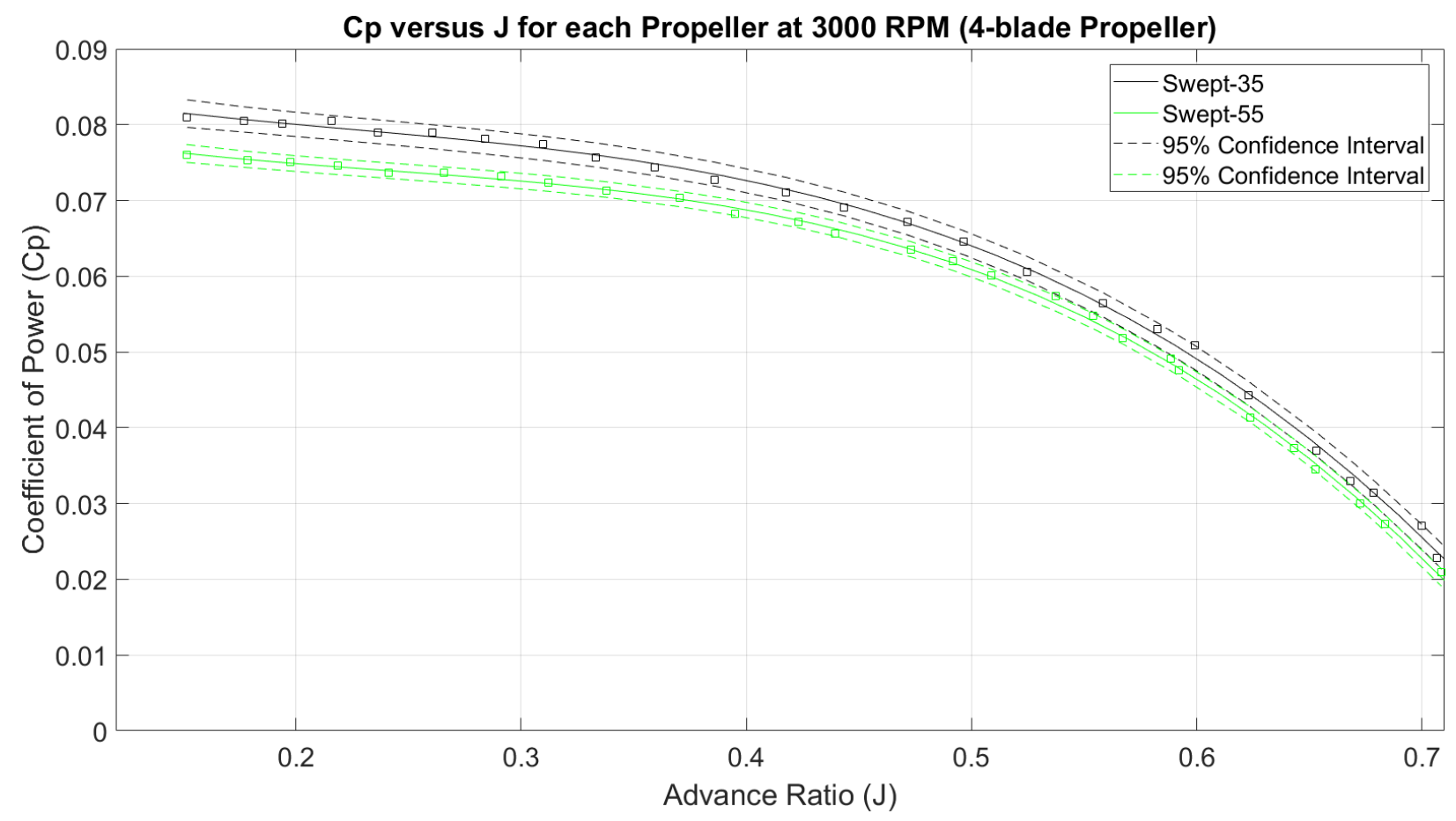

Figure 73: Coefficient of Thrust plot for the Swept-35 and Swept-55 4bladed propellers. 


\subsection{EXPERIMENTAL ACOUSTIC RESULTS (OUTDOORS)}

The background noise recorded at various points during the morning are plotted in figure 74 and presented as $\mathrm{dBA}$. Any value below $0 \mathrm{dBA}$ is a pressure variation below the referenced value, $\mathrm{Pa}_{\mathrm{Ref}}=20 \times 10^{-6}$ $\mathrm{Pa}$, which is the limit of sensitivity of the human ear. Thus, an SPL at or below $0 \mathrm{dBA}$ still exists as a pressure wave but is not perceived by the ear. These negative $d B A^{\prime}$ s have been included to show the entire spectrum of ambient noise during the test day. As expected, the background noise is smallest in the morning and the winds, even gradual, contribute to noise significantly in the lower frequencies. Figure 75 magnifies the low frequency spectrum $(0-1 \mathrm{kHz})$ and highlights the variation in background noise. Even with these variation in noise, these conditions were still acceptable for testing. The motor was also tested in isolation and compared to the background noise in figure 76. All plots used the built-in MATLAB function pwelch for postprocessing.

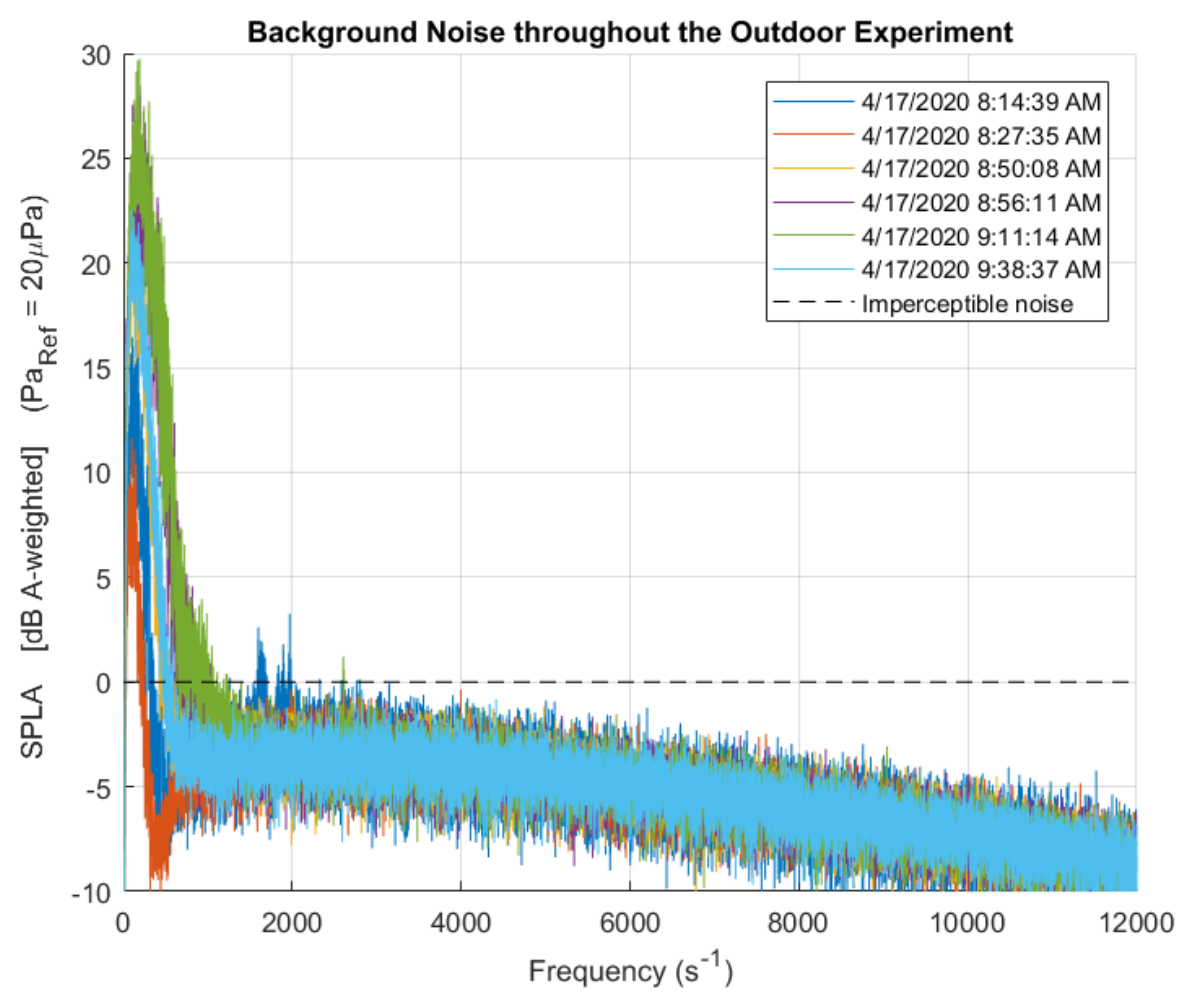

Figure 74: Background noise at various point of the day. It is not necessary to plot the negative dB but a "wide view" of the entire spectrum shows that there is no audible noise above $2 \mathrm{KHz}$. 


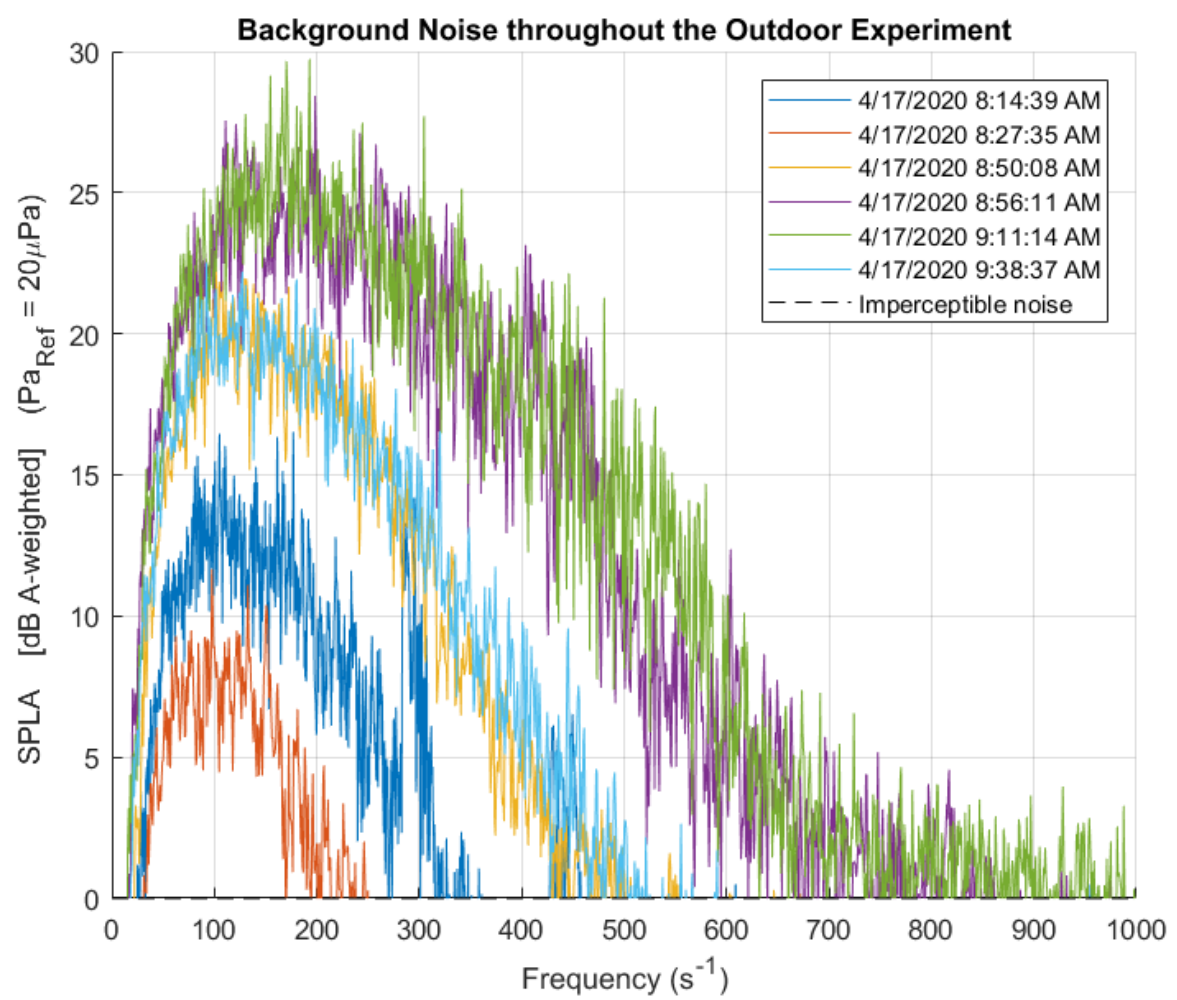

Figure 75: Detailed view of the low frequency range from figure 74.

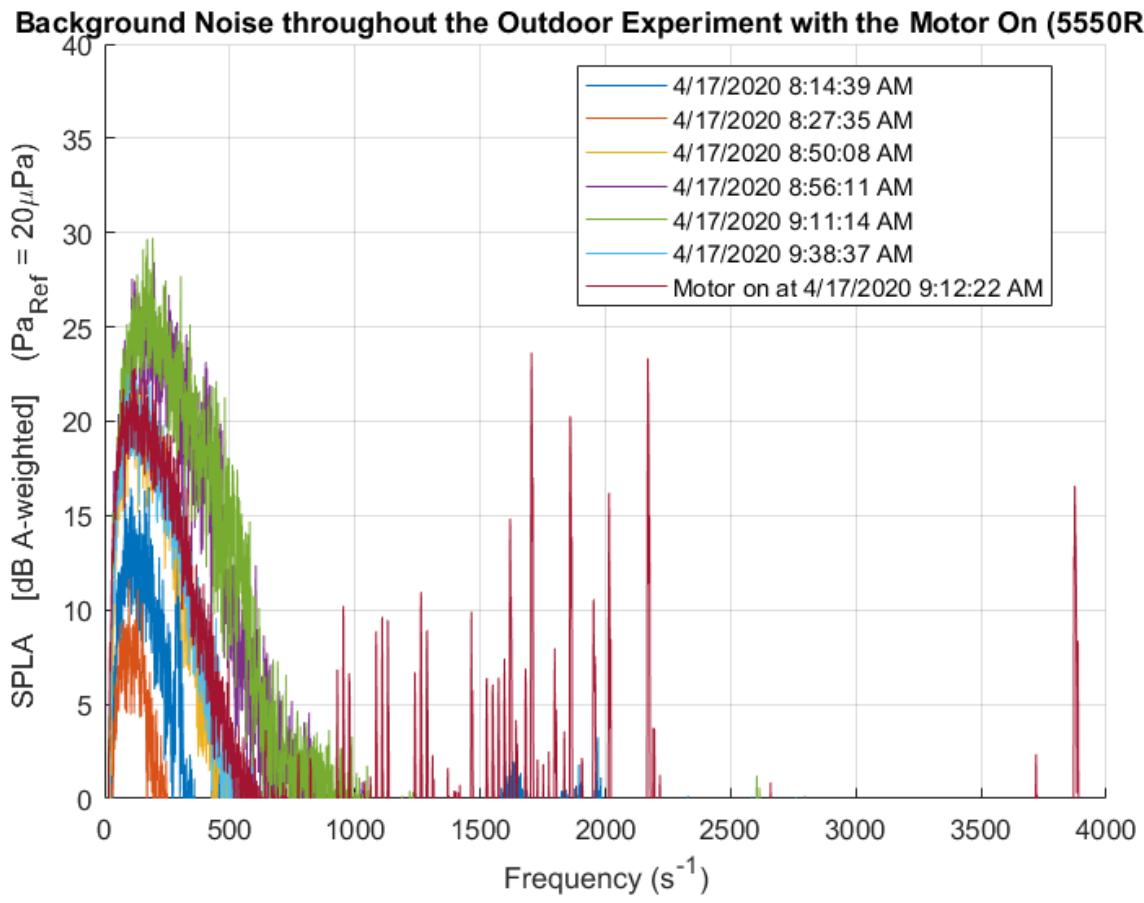

Figure 76: Background noise compared to the motor noise at 5550 RPM 
Figure 77, 78, and 79 show the swept-15, swept-35, and swept-55 propeller acoustic spectrum at 5550 RPM. Each propeller was recorded at three different runs over a duration of 15 seconds starting at the timestamp included in the figures. The trends continued out to higher frequencies, but the tonal noise is the most important aspect to investigate. The first blade passing frequency (BPF) occurs at $\sim 185 \mathrm{~Hz}$, and the BPF harmonics occur at their respective locations. The runs would start when the wind was calm but due to the nature of testing outdoors, the wind would often suddenly pick up and contribute additional noise. The repeated runs were implemented as a means of examining how significantly the wind impacted the propeller data as well as to increase the likelihood that at least one run had little to no wind during the observation. The background noise figures. Figures 74 and 75 , shed light onto which propeller runs had minimal external noise caused by the environment. In figures 77,78 , and 79 the runs varied mainly in the lower frequencies $(100-500 \mathrm{~Hz})$ where the addition of wind compounds the noise in this range. It can be concluded that the runs with the least amount of continuous noise with respect to the BPF in the low frequency range are the "best" run for each propeller. These runs were used in the comparison plot in figure 80 . In all the runs, the first BPF occurs at $\sim 185 \mathrm{~Hz}$ and the additional peaks that occur are integer multiples of the first BPF called the harmonic BPF; however, only the first few harmonic BPF are discernible from the continuous noise. The BPF is calculate with equation (38).

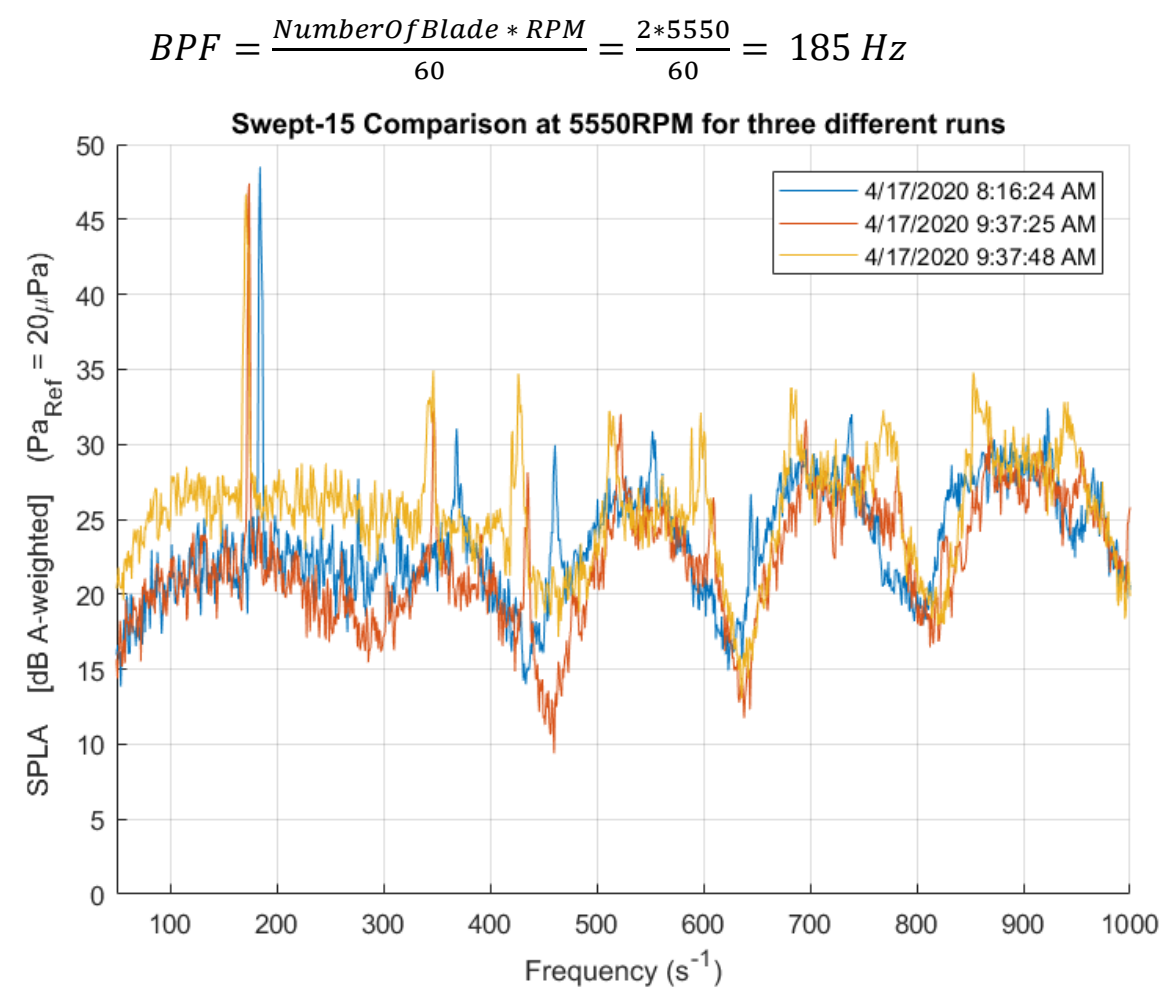

Figure 77: Swep-15 evaluated at three different runs. ( $r=9^{*} D, 45^{\circ}$ below) 


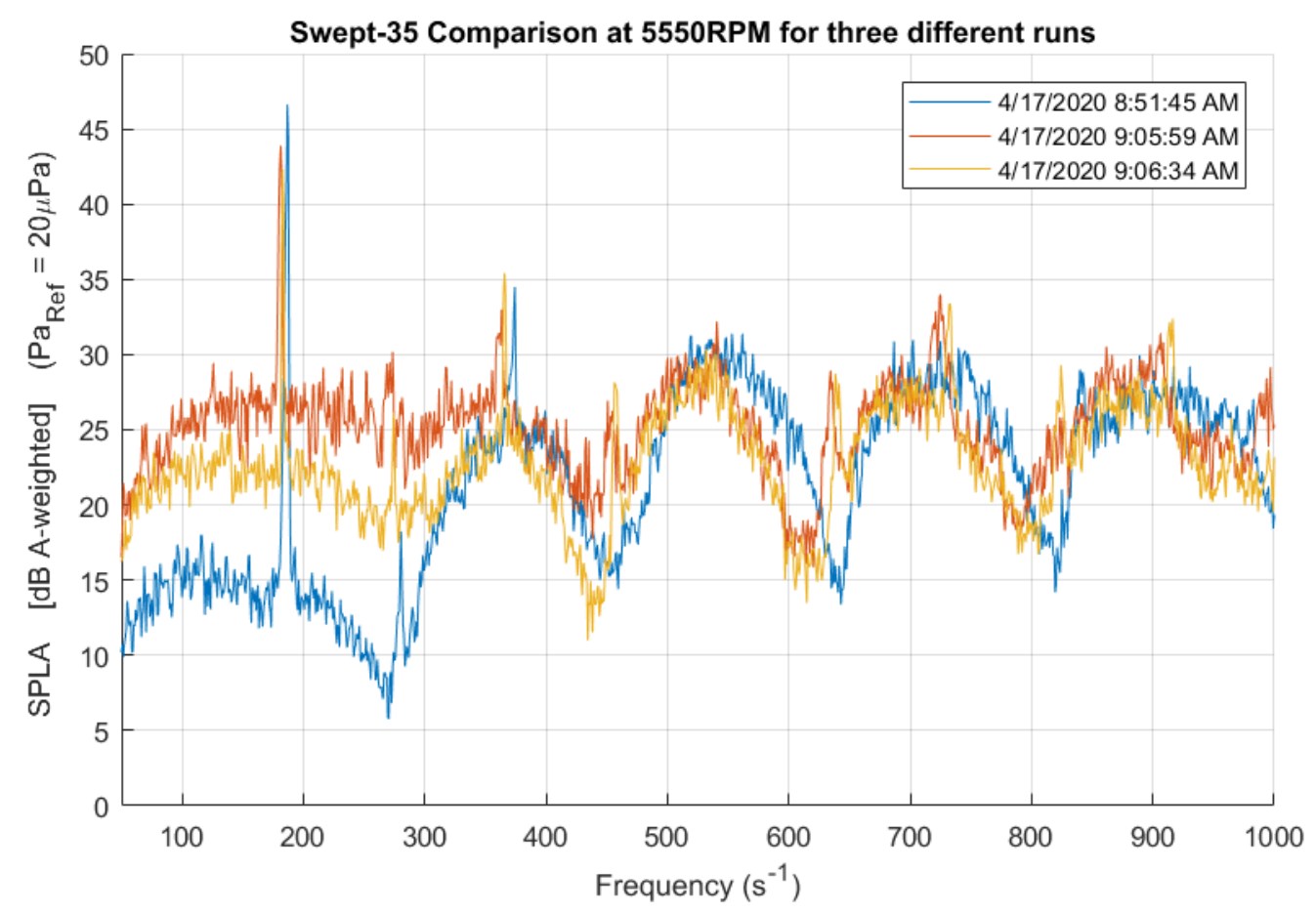

Figure 78: Swept-35 evaluated at three different runs ( $r=9 * D, 45^{\circ}$ below)

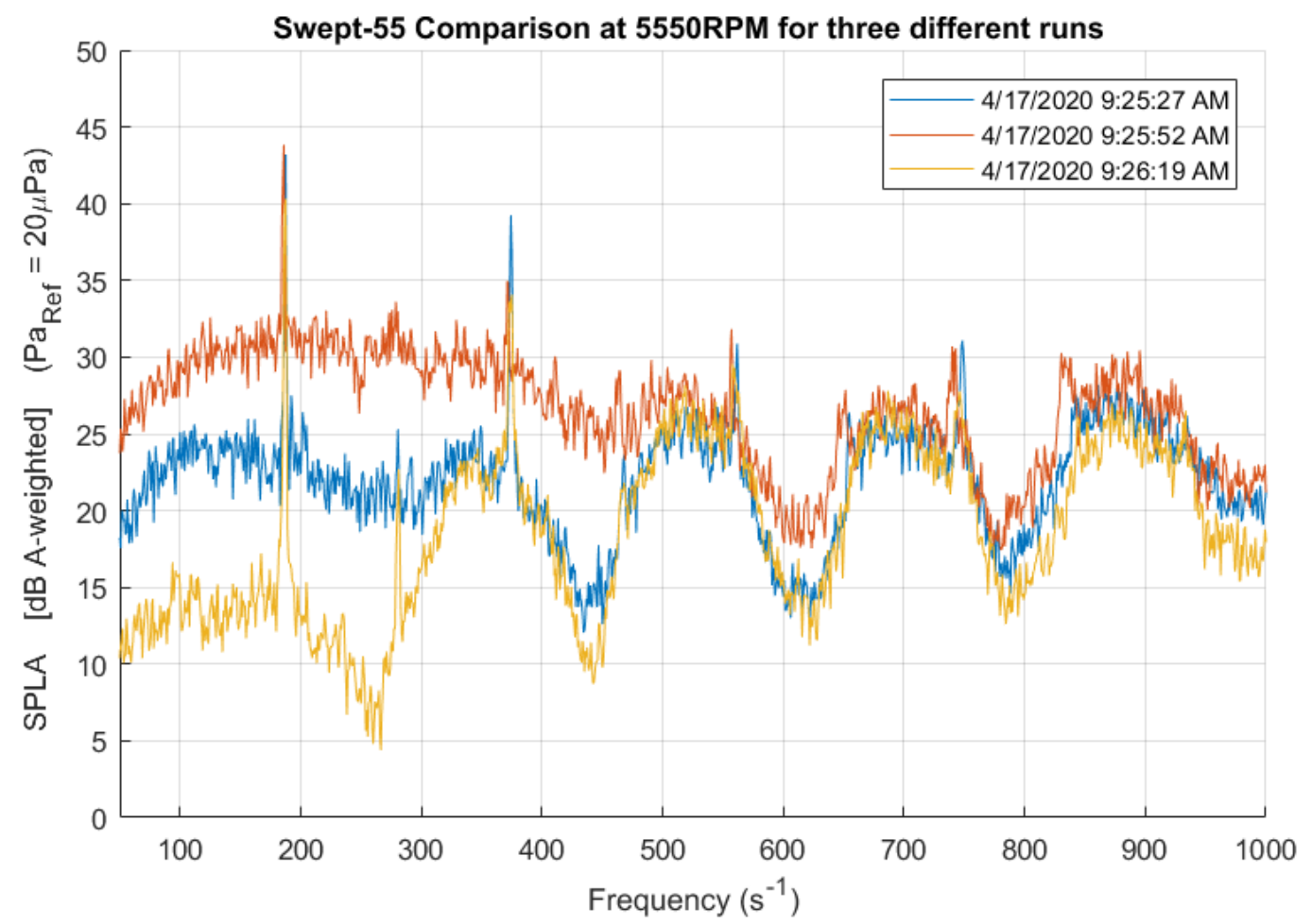

Figure 79: Swept-55 evaluated at three different runs $\left(r=9 * D, 45^{\circ}\right.$ below) 
The comparison plot shows the "best" case for the four different blade designs under study. The most apparent difference between the four designs is the continuous noise in the lower frequencies. This highlights the impact of the environment on the swept-15 case (in blue) versus the clean run of the straight propeller (in purple). Figure 81 shows a zoomed in graph of the first BPF comparison. This figure highlights the difference in $\mathrm{dBA}$ between the various designs. The swept-15 peaks at $48 \mathrm{dBA}$, while the swept-35 and straight propeller differ only minutely from one another. The swept-55 propeller has the lowest sound pressure level of $40 \mathrm{dBA}$, but figure 79 shows that this run produced lower $\mathrm{dB}$ than the other two runs of the same design and RPM, which were taken only seconds apart from one another. Those runs produced similar tonal dBA to the swept-35 and straight design seen in the comparison figure. This suggests that the dBA confidence intervals overlap between designs.

Figure 82 uses MATLAB's bandpower function to calculate the average power near the first five BPF. A $\pm 6 \mathrm{~Hz}$ range was given to each peak, to ensure that the tonal peaks were captured for each propeller. The figure suggests that the swept designs produces weaker Harmonic BPF and this trend is systematic for higher frequencies. However, no definitive conclusions can be made for this data since this was a single outdoor run on a mostly calm day, which, even under ideal weather and setting, produced notable background noise as the experiment was conducted throughout the morning. One of two methods can be taken to validate this data. Either several additional outdoor runs can be conducted under similar circumstances, or this test can be conducted in an anechoic chamber where these external variables are controlled. 


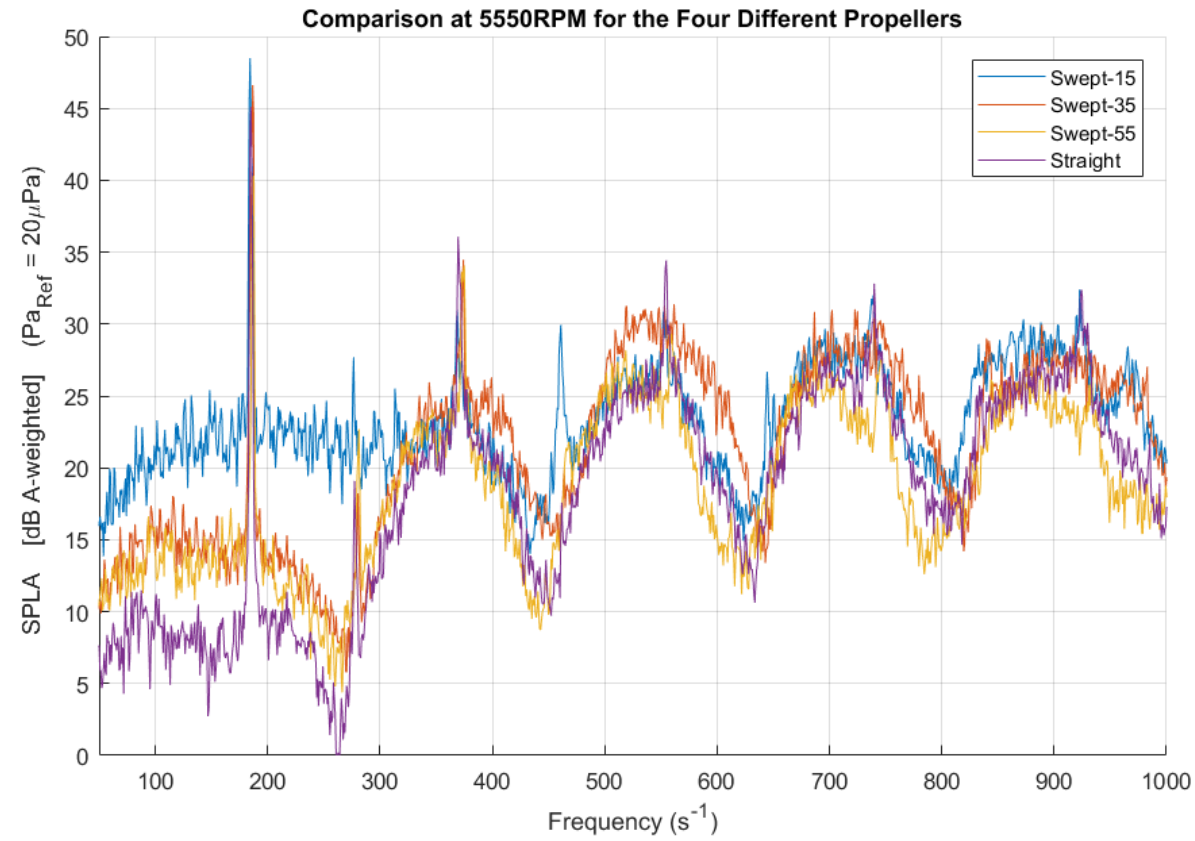

Figure 80: Comparison between the four different propellers for each "best" case.

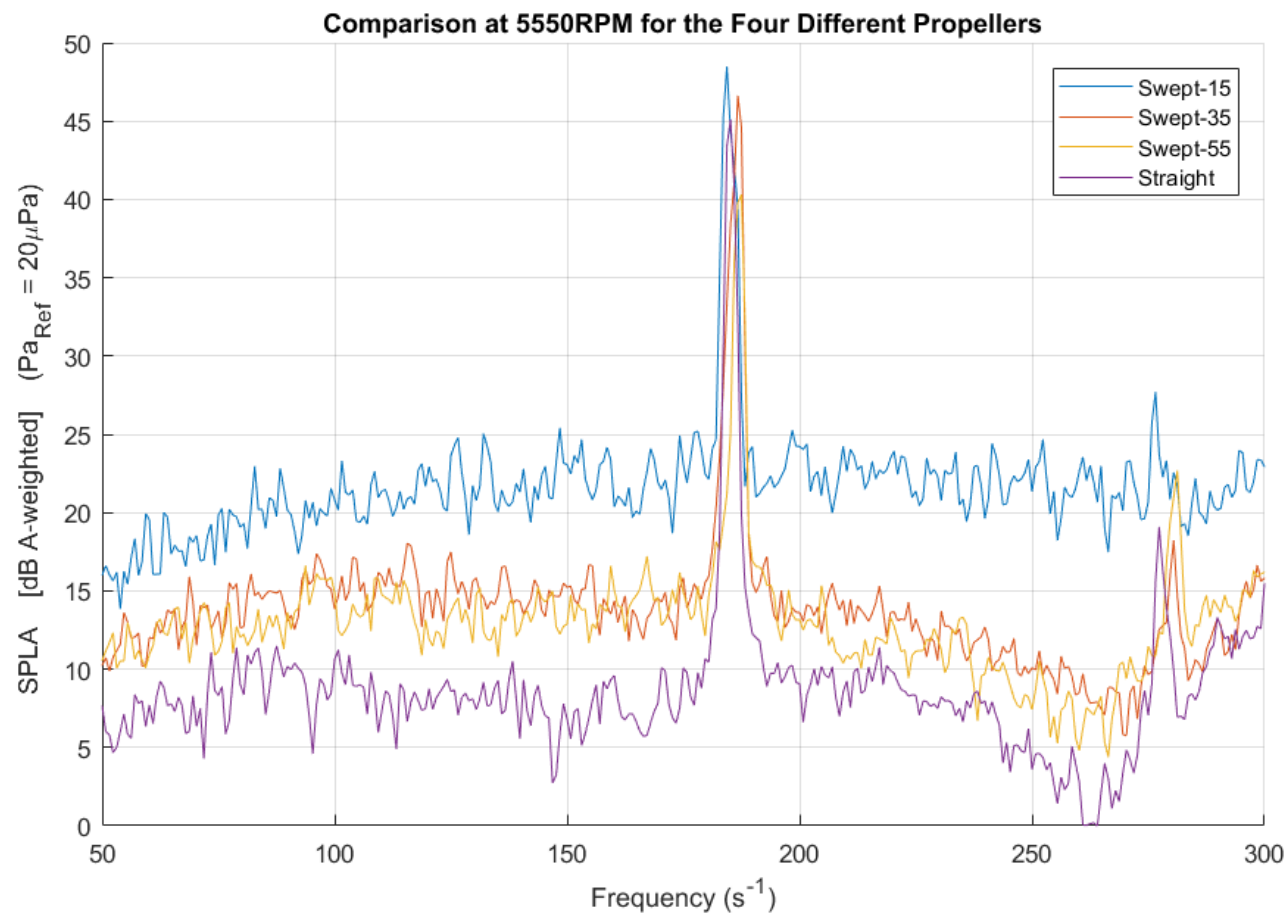

Figure 81: Close up comparison of the first BPF. 


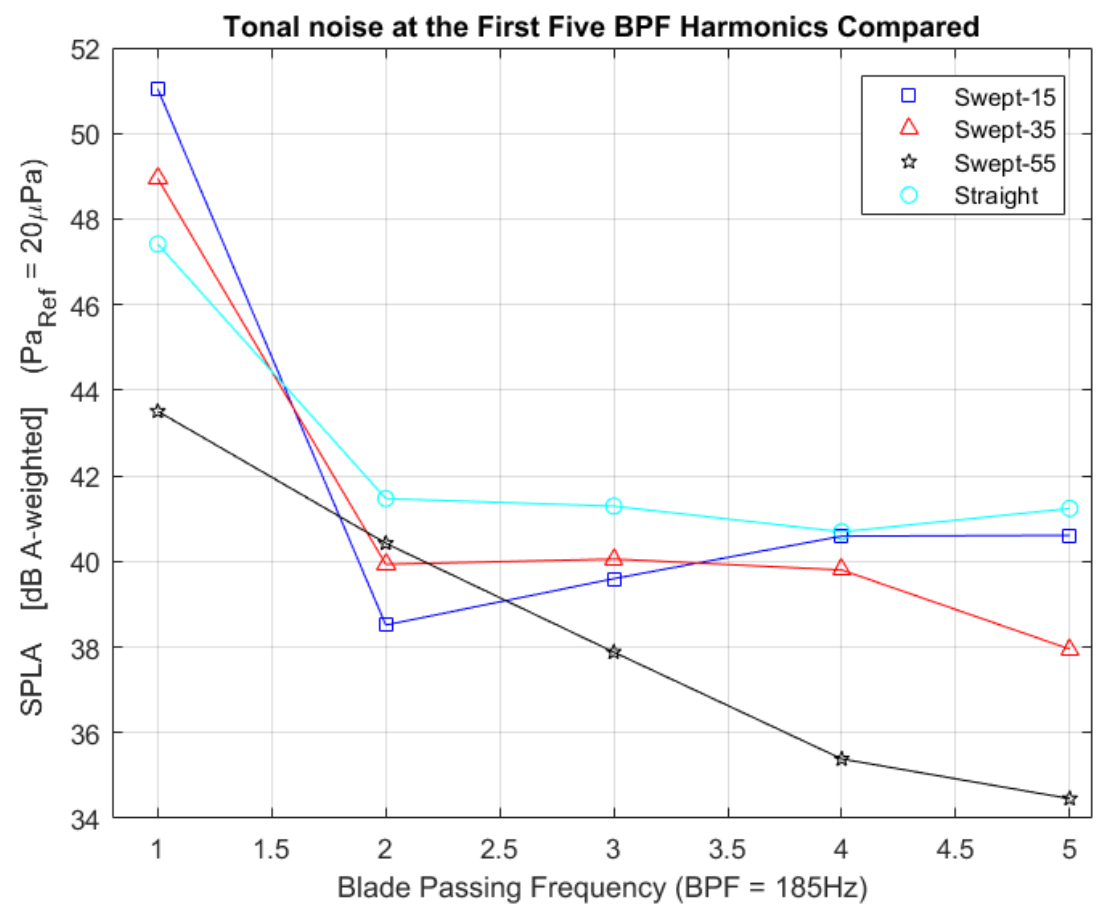

Figure 82: Comparison between the first five BPF harmonics between blade designs. 


\section{CHAPTER 8 CONCLUSION AND FUTURE WORK}

This study investigated the aerodynamic and aeroacoustic properties of several unconventional propeller designs against a straight propeller blade intended for a small UAV. These propellers were made of Aluminum 6061 and were manufactured with a CNC milling machine with two different cutting methods. Of the two methods used, the 3DOF milling method produced the more consistent finish across the various propeller designs constructed. The aerodynamics was evaluated and while the coefficient of thrust and torque varied slightly from one propeller design to another, their differences ultimately canceled out in the efficiency plots. The aerodynamic efficiency a propeller is not significantly altered by the addition of sweep, even when the propeller has a dramatic sweep angle. This suggests the propeller blade's sweep can be altered to find an optimum design to minimize the acoustic noise without hampering the aerodynamic performance. For the aeroacoustic side of the investigation, a computational and experimental method was employed to investigate the tonal differences between a conventional and swept propeller. ANOPP2-F1A was used to evaluate the pressure signal across a wide range of RPMs, distances, and observer angles to generate trends to investigate each of the propeller's waveforms. The computational results showed little difference between the designs and suggested the swept designs were slightly louder, likely a result of integrating along a longer chord length. Despite this, ANOPP2 shows a swept blade can alter the pressure waveform. This means that there is potential to create maximum destructive interference between the loading and thickness noise. The aeroacoustic experiment was conducted outdoors on a calm day but, even under good conditions, definitive results cannot be established due to the variation in background noise between runs, but, even with the noisy data, the experiment suggests the more dramatic swept designs produce less noise.

Additional runs are planned for the outdoor run to validate and expand the dataset. The dataset will be expanded by evaluating with more observer angles, observers placed in both near and far field, higher blade count, and various operating RPM for all blade designs. A similar test will be run in an anechoic chamber. The aerodynamic will be conducted again with a spinner placed on the hub. The spinner should result in a small but noticeable decrease in $C_{p}$ and an overall increase in performance. 


\section{REFERENCES}

[1] Y. Huang, K. N. Reddy, R. S. Fletcher, and D. Pennington, "UAV Low-Altitude Remote Sensing for Precision Weed Management," Weed Technology, vol. 32, no. 1, pp. 2-6, 2018, doi:

10.1017/wet.2017.89.

[2] National Park Service, Independence National Historical Park Archives., "Understanding Sound," July 3, 2018, https://www.nps.gov/subjects/sound/understandingsound.htm

[3] R. Brigham, E. Kalko, G. Jones, S. Parsons, and H. Limpens, "Bat echolocation research: tools, techniques and analysis," Bat Conservation International. Austin, Texas, 2004.

[4] D. W. Robinson and R. S. Dadson, "A re-determination of the equal-loudness relations for pure tones," British Journal of Applied Physics, vol. 7, no. 5, p. 166, 1956.

[5] Y. Suzuki et al., "Precise and full-range determination of two-dimensional equal loudness contours," Tohoku University, Japan, 2003.

[6] B. E. Duvall, "Development and implementation of a propeller test capability for GL-10" Greased Lightning" propeller design," 2016.

[7] A. W. Vogeley, "Sound-level measurements of a light airplane modified to reduce noise reaching the ground," 1949.

[8] W. Harlamert, "A Quiet Propeller for Commuter and General Aviation Aircraft," SAE Technical Paper, 0148-7191, 1974.

[9] R. K. Rathgeber and D. E. Sipes, "The Influence of Design Parameters on Light Propeller Aircraft Noise," SAE Technical Paper, 0148-7191, 1977.

[10] A. Lee and M. Mosher, "A study of the noise radiation from four helicopter rotor blades [tests in Ames 40 by 20-foot wind tunnel]," 1978.

[11] D. Davis, "The Impact of Noise Regulations on Propeller Design," SAE Transactions, pp. 2086-2095, 1979

[12] R. J. Klatte and F. B. Metzger, "Influence of Noise Reduction on Weight and Cost of General Aviation Propellers," UNITED TECHNOLOGIES CORP WINDSOR LOCKS CONN HAMILTON STANDARD DIV, 1979

[13] W. Dobrzynski, "The effect on radiated noise of non-zero propeller rotational plane attitude," in 10th Aeroacoustics Conference, 1986, p. 1926.

[14] F. Weiblen, "A contribution to noise improvements for aircraft by noise measurement evaluation," in 14th DGLR/AIAA Aeroacoustics Conference, 1992, vol. 2, pp. 623-627.

[15] M. Chusseau, S. Pauzin, and R. Roozen, Light Aircraft propeller: Design Parameter Effects on Acoustics and Aerodynamics. AIAA, 1993. 
[16] R. D. Hager, Advanced turboprop project. Scientific and Technical Information Division, National Aeronautics and Space Administration, 1988.

[17] T. Kim, "Reduction of tonal propeller noise by means of uneven blade spacing," UC Irvine, 2016.

[18] D. L. Huff and B. S. Henderson, "Electric Motor Noise for Small Quadcopters: Part 1-Acoustic Measurements," in 2018 AIAA/CEAS Aeroacoustics Conference, 2018, p. 2952.

[19] gZawodny, N. S. and Boyd Jr., D. D., "Investigation of Rotor-Airframe Interaction Noise Associated with SmallScale Rotary-Wing Unmanned Aircraft Systems," AHS International 73rd Annual Forum, 2017

[20] D. B. Hanson, "Influence of propeller design parameters on far-field harmonic noise in forward flight," AIAA Journal, vol. 18, no. 11, pp. 1313-1319, 1980.

[21] Brandt, J. B., "Small-Scale Propeller Performance at Low Speeds," M.S. Thesis, Department of Aerospace Engineering, University of Illinois at Urbana-Champaign, Illinois, 2005.

[22] M. K. Rwigema, "Propeller blade element momentum theory with vortex wake deflection," in 27th International congress of the aeronautical sciences, 2010, vol. 2010, p. 2.3.

[23] ANOPP2 Development Team, “ANOPP2 Functional Module Manual”, NASA, Version 1.2

[24] N. L. Schatzman, "Aerodynamics and Aeroacoustic Sources of a Coaxial Rotor," Ph. D. thesis, Georgia Institute of Technology, Atlanta, GA, 2018.

[25] F. Farassat, "Derivation of Formulations 1 and 1A of Farassat," 2007.

[26] K. S. Brentner and F. Farassat, "An analytical comparison of the acoustic analogy and Kirchhoff formulation for moving surfaces," 1997.

[27] The ANOPP2 Development Team, "ANOPP2 Functional Module Manual Version 1.2", NASA Langley Research Center, Hampton, VA, April 2019

[28] K. S. Brentner and F. Farassat, "Modeling aerodynamically generated sound of helicopter rotors," Progress in Aerospace Sciences, vol. 39, no. 2-3, pp. 83-120, 2003.

[29] Drela M., Youngren H., "XROTOR User Guide”, Massachusetts Institute of Technology, Retrieved from http://web.mit.edu/drela/Public/web/xrotor/xrotor doc.txt, 2003

[30] L. Lopes and C. Burley, "Design of the next generation aircraft noise prediction program: ANOPP2," in 17th AIAA/CEAS Aeroacoustics Conference (32nd AIAA Aeroacoustics Conference), 2011, p. 2854.

[31] A. Wiedemann, "Step-by-Step guide to making a NACA rotor blade," unpublished

[32] Sadowski, Nicholas B. "A Cost Effective Design for a Propeller Thrust/Torque Balance" (2018). Master of Science (MS), thesis, Mechanical \& Aerospace Engineering, Old Dominion University, DOI: 10.25777/f8mc-9c26 https://digitalcommons.odu.edu/mae etds/39 
[33] Benjamin, Colin B. "Characterization and Optimization of a Propeller Test Stand" (2019). Master of Science (MS), Thesis, Mechanical \& Aerospace Engineering, Old Dominion University, DOI:

10.25777/8zmp-r426 https://digitalcommons.odu.edu/mae etds/198 


\section{APPENDIX A. MATLAB CODE DEFINING A SWEPT PROPELLER}

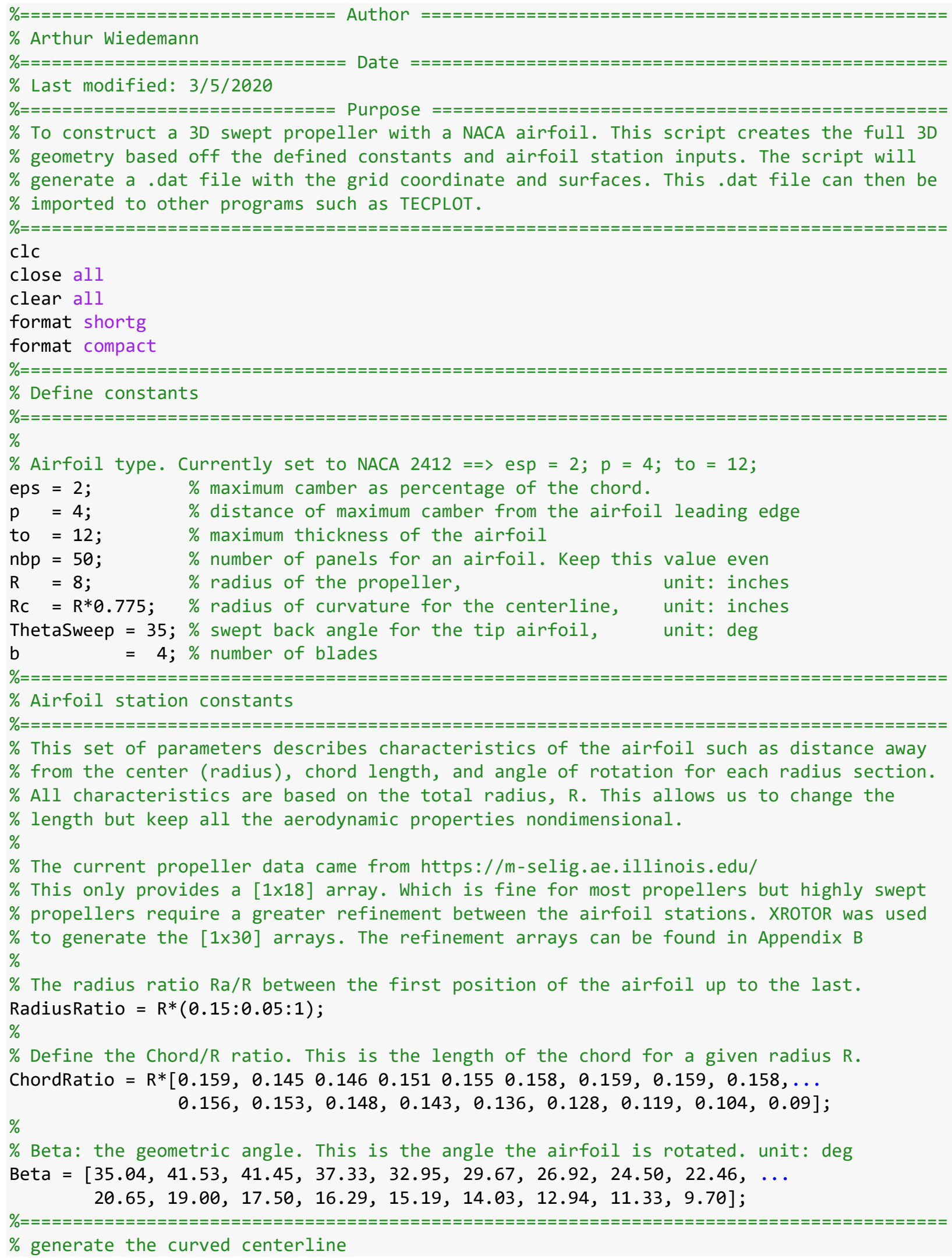




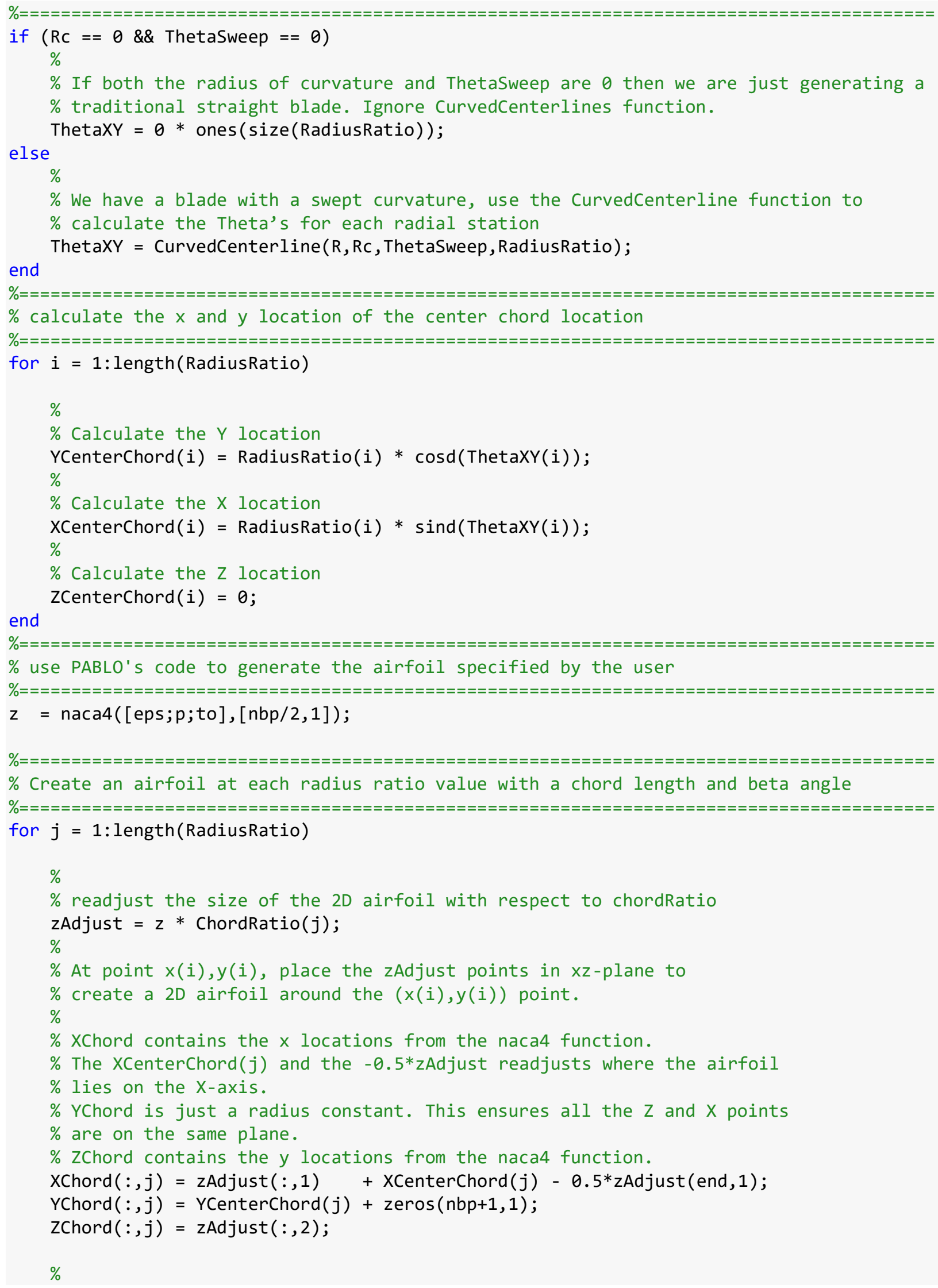


$\%$ Rotate the airfoil in the $x z-p l a n e$ using the Beta angle

for $i=1:(n b p+1)$

$\%$

$\%$ Use the rotation matrix to rotate the airfoil based on the current

$\%$ Beta angle input. The XCenterChord/ZCenterChord are used to

$\%$ correctly position the values in 3D space.

BetaRotate $=$

$[$ XCenterChord $(j)$; ZCenterChord $(j)] \quad+\ldots$

$[\operatorname{cosd}(\operatorname{Beta}(j)), \operatorname{sind}(\operatorname{Beta}(j)) ;-\operatorname{sind}(\operatorname{Beta}(j)), \operatorname{cosd}(\operatorname{Beta}(j))] * \ldots$

$[(\operatorname{XChord}(i, j)-X C e n t e r C h o r d(j)) ;(\operatorname{ZChord}(i, j)-$ ZCenterChord $(j))]$;

$\%$

$\%$ The Reposition variables are a [1×2] where the top value is the new

$\% \mathrm{x}$ and the bottom value the new $\mathrm{z}$. Assign them to their proper

$\%$ place.

$x \operatorname{Chord}(i, j)=$ BetaRotate $(1,1)$;

$\operatorname{zChord}(i, j)=$ BetaRotate $(2,1)$;

end

$\%$

$\%$ The angle the airfoil needs to be normal to the curved centerline. This is based

$\%$ off the local theta. The negative sign rotates the system $\mathrm{cw}$ instead of $\mathrm{ccw}$

Angle $=-\operatorname{ThetaXY}(j)$;

$\%$

\% Rotate the airfoil in the xy-plane using the "-ThetaXY" angle

for $i=1:(n b p+1)$

$\%$

$\%$ Reposition each airfoil based off the "-ThetaXY" Angle.

$\%$ This must be done to each grid point on the current airfoil.

Reposition $=$

[XCenterChord $(j)$; YCenterChord $(j)]$

[cosd(Angle), -sind(Angle); sind(Angle), cosd(Angle) $] \quad * .$.

$[(X C h o r d(i, j)-X C e n t e r C h o r d(j)) ; \quad(Y C h o r d(i, j)-Y C e n t e r C h o r d(j))]$;

$\%$

$\%$ The Reposition variables are a [1x2] where the top value is the new

$\% \mathrm{x}$ and the bottom value the new $\mathrm{y}$. Assign them to their proper place

$\operatorname{xChord}(i, j)=\operatorname{Reposition}(1,1)$;

$\operatorname{YChord}(i, j)=\operatorname{Reposition}(2,1)$;

end

end

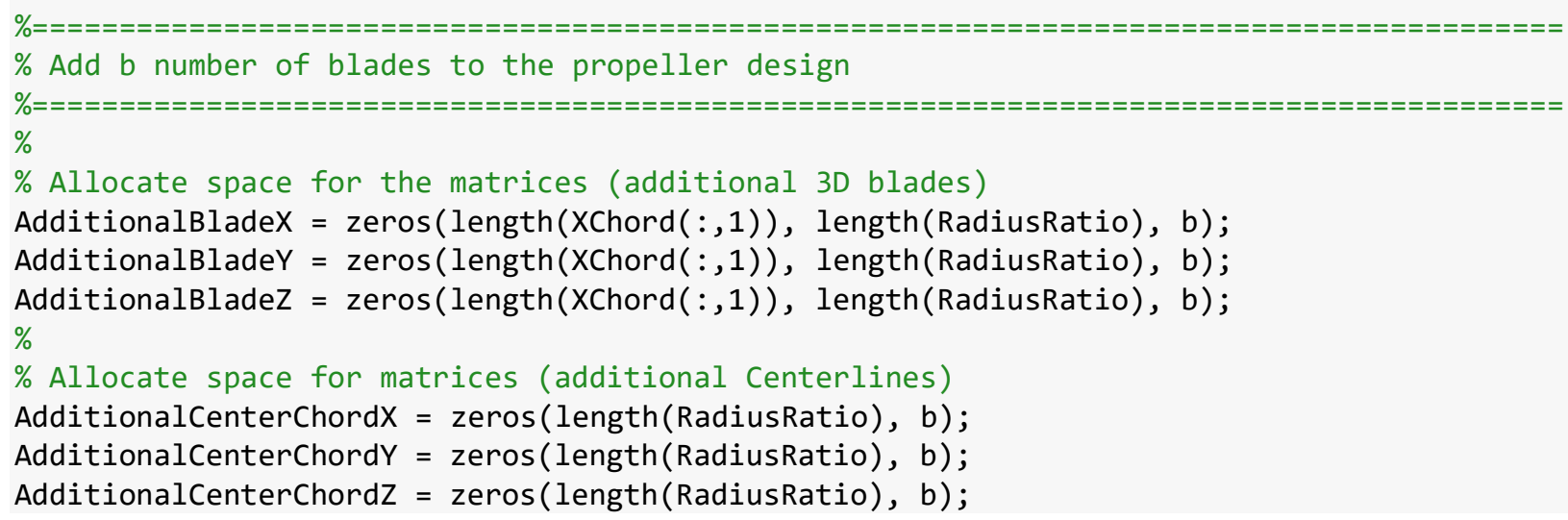




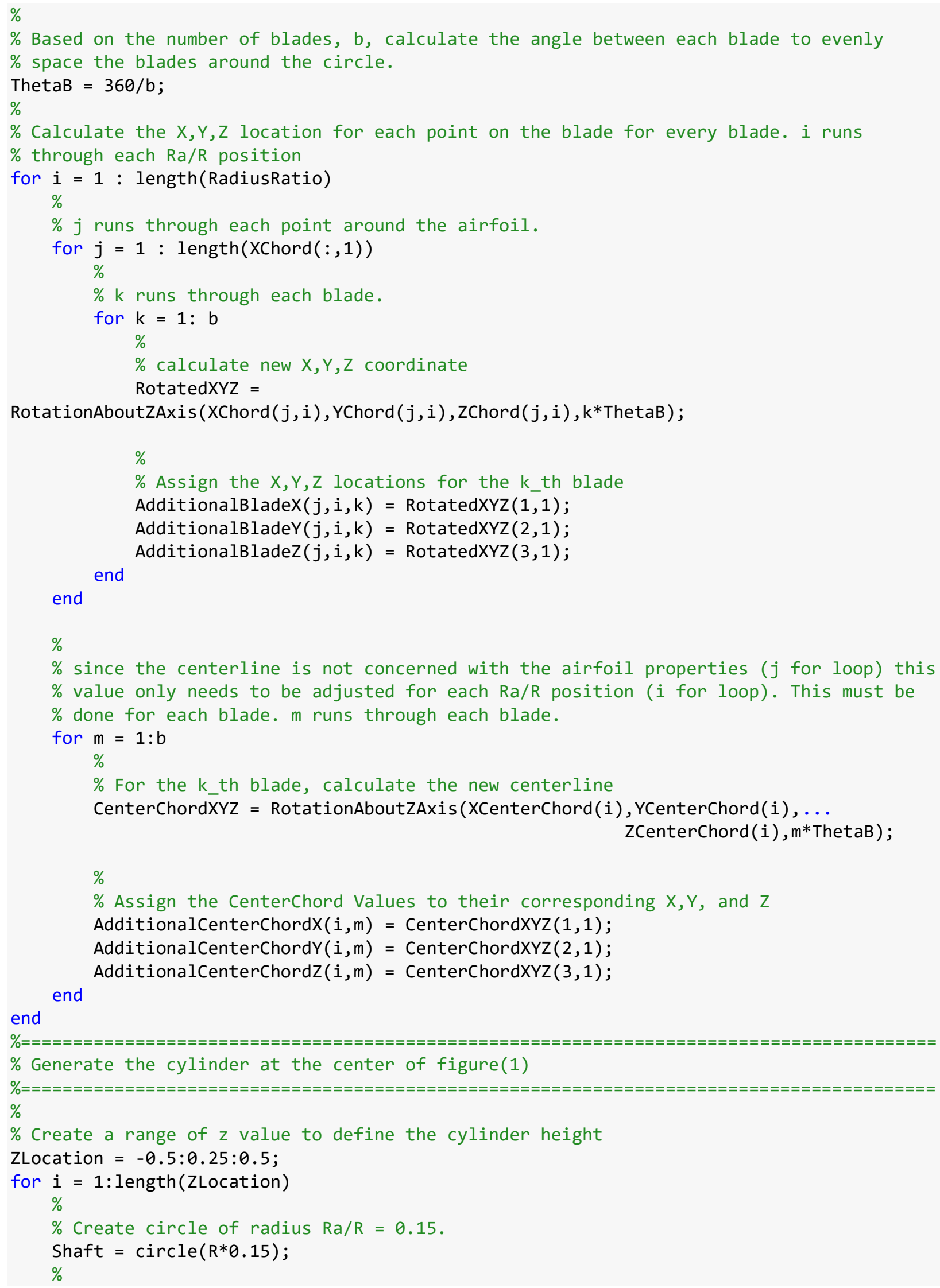




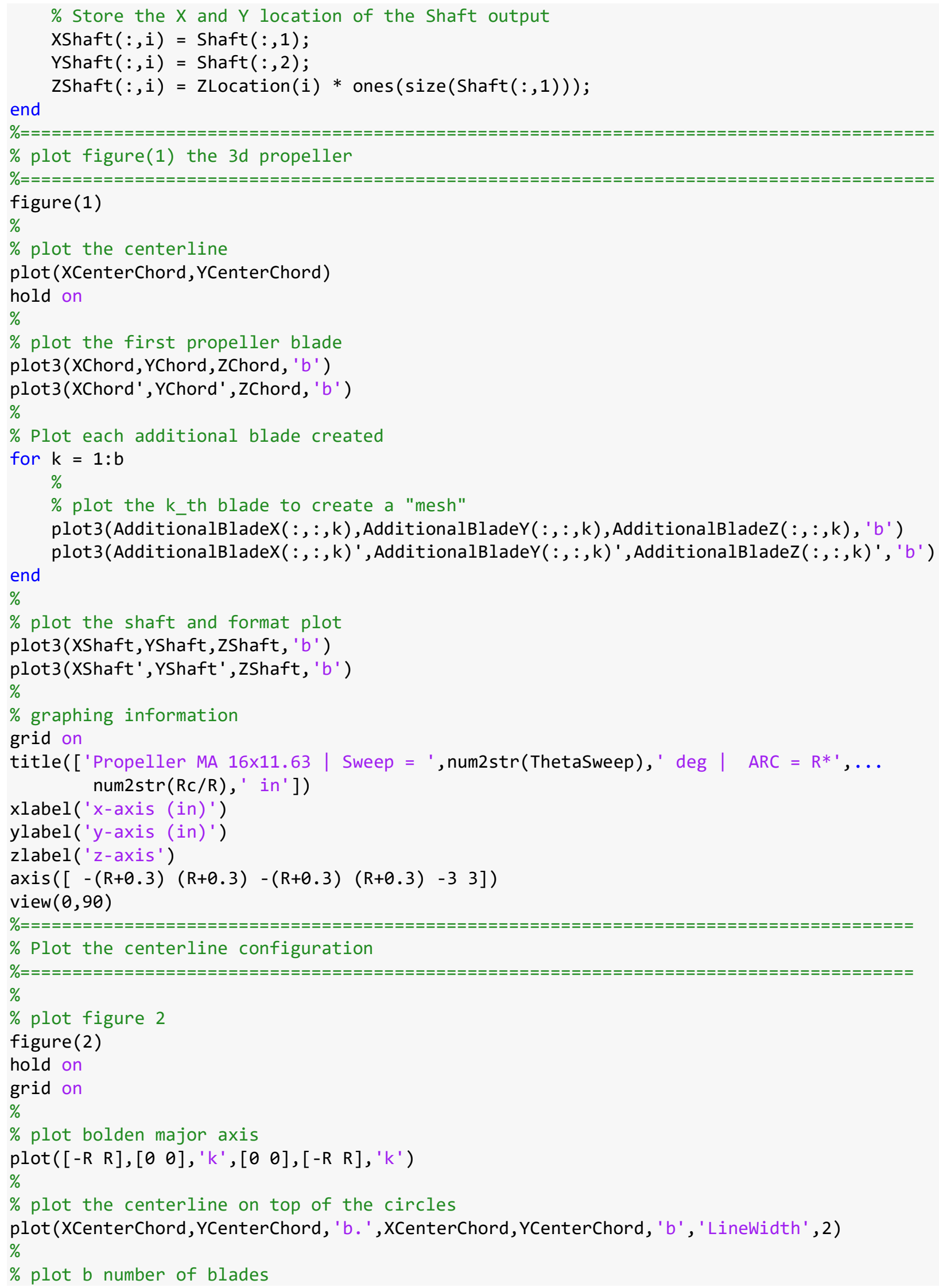




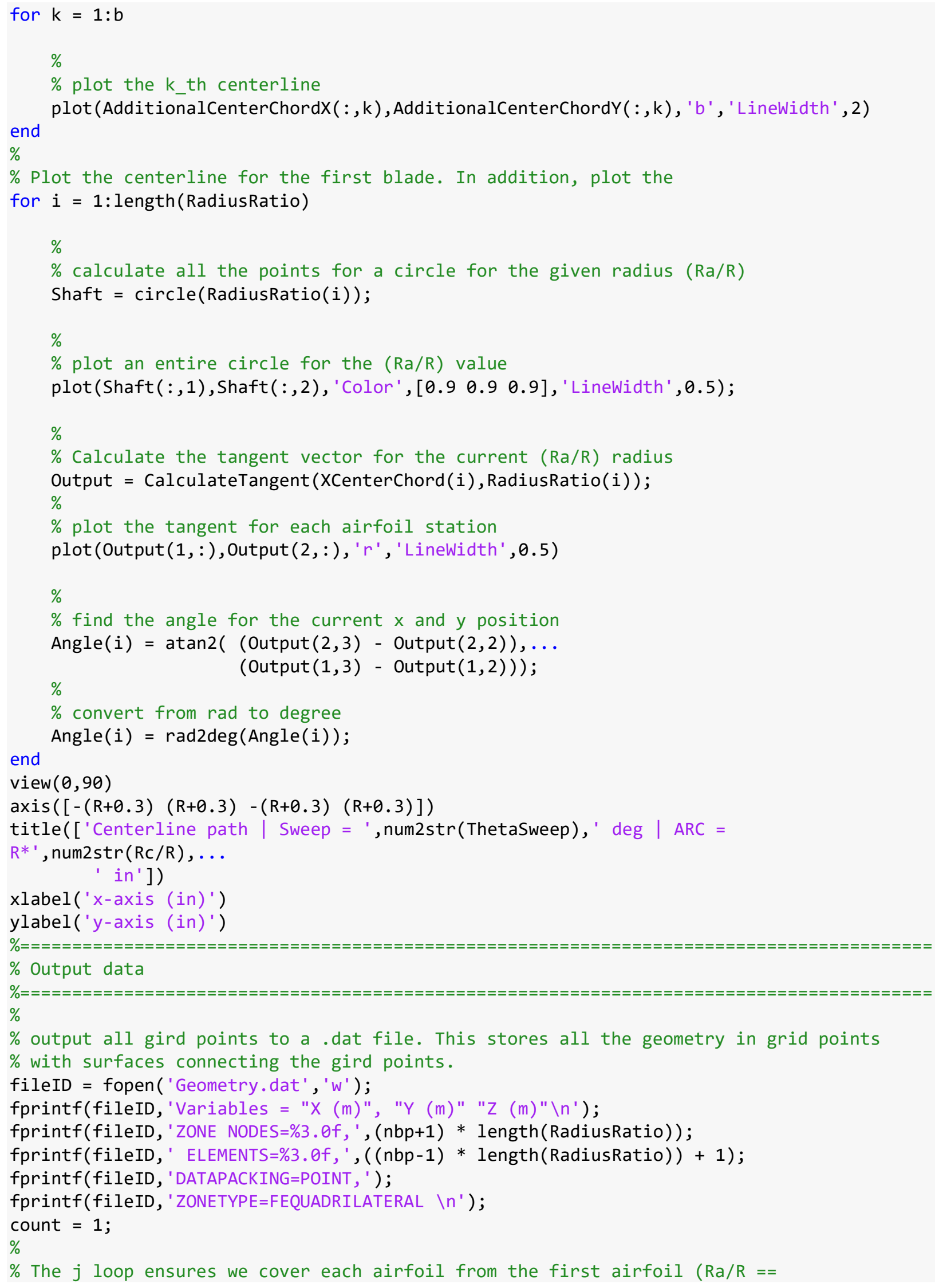


$\% 0.15)$ to the last airfoil $(\mathrm{Ra} / \mathrm{R}==1)$.

for $j=1$ : length $(\operatorname{XChord}(1,:))$

$\%$

\% The i loop ensures we output each datapoint inside an individual

$\%$ airfoil. (so, all the points that make up an airfoil are outputted

$\%$ with the inner loop)

for $i=1$ : length $(X \operatorname{Chord}(:, 1))$

$\%$

$\%$ Output the current point $X, Y, Z$ location in 3D space to the file

fprintf(fileID, '\%6.4f \%6.4f \%6.4f $\backslash n^{\prime}$, $\operatorname{xChord}(i, j)$, YChord(i,j), ...

end

$\operatorname{ZChord}(i, j))$;

end

for $j=1$ : length $(X \operatorname{Chord}(1,:))$

for $i=1$ : length $(\operatorname{XChord}(:, 1))$

$\operatorname{node}(i,:)=((j-1) * n b p)+[(i), n b p+1+i, i+n b p+2, i+1] ;$

fprintf(fileID, '\%2. of \%2. of $\% 2.0 f \quad \% 2.0 f \backslash n '$, node $(i,:))$;

$\%$

$\%$ Once the largest integer in the node output hits the maximum

$\%$ number of points $((X, Y, Z)$ locations) we are finished assigning

$\%$ node locations

if $(\operatorname{node}(i, 3)==((n b p+1) *$ length $($ RadiusRatio $)))$

$\%$

$\%$ once we compute all the nodes, break out of the for loops break;

end

end

end

fclose(fileID);

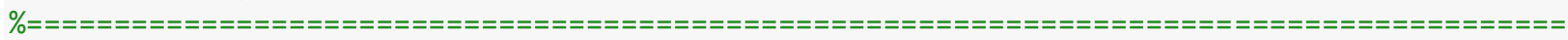

$\%$ 1st Function

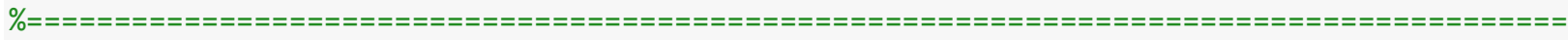

function XandYPointsForCircle $=$ circle(radius)

$\%$

$\%$ create a range from $\theta$ to 2 pi. This will be use to calculate each point

$\%$ along the circle

ThetaRange $=0: 4: 360$;

for $i=1:$ length (ThetaRange)

$\%$

\% Calculate the $\mathrm{x}$ position at the current theta angle

$X$ Point $(i)=\operatorname{cosd}($ ThetaRange $(i)) *$ radius;

$\%$

\% Calculate the y position at the current theta angle

YPoint $(i)=\operatorname{sind}($ ThetaRange $(i)) *$ radius;

end

$\%$

$\%$ Store all values in the output so it can be plotted later

XandYPointsForCircle $(:, 1)=$ XPoint;

XandYPointsForCircle $(:, 2)=$ YPoint; 


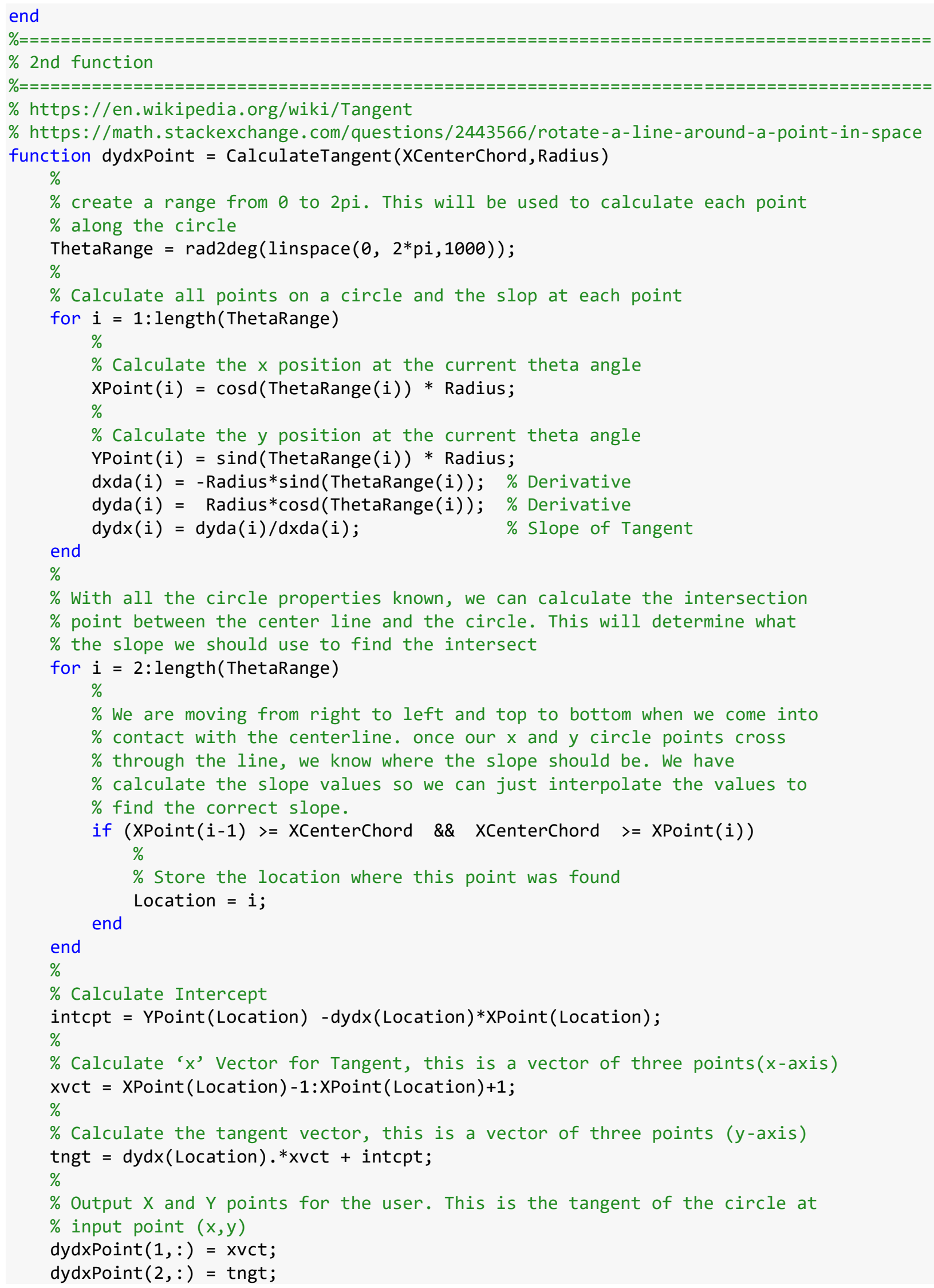




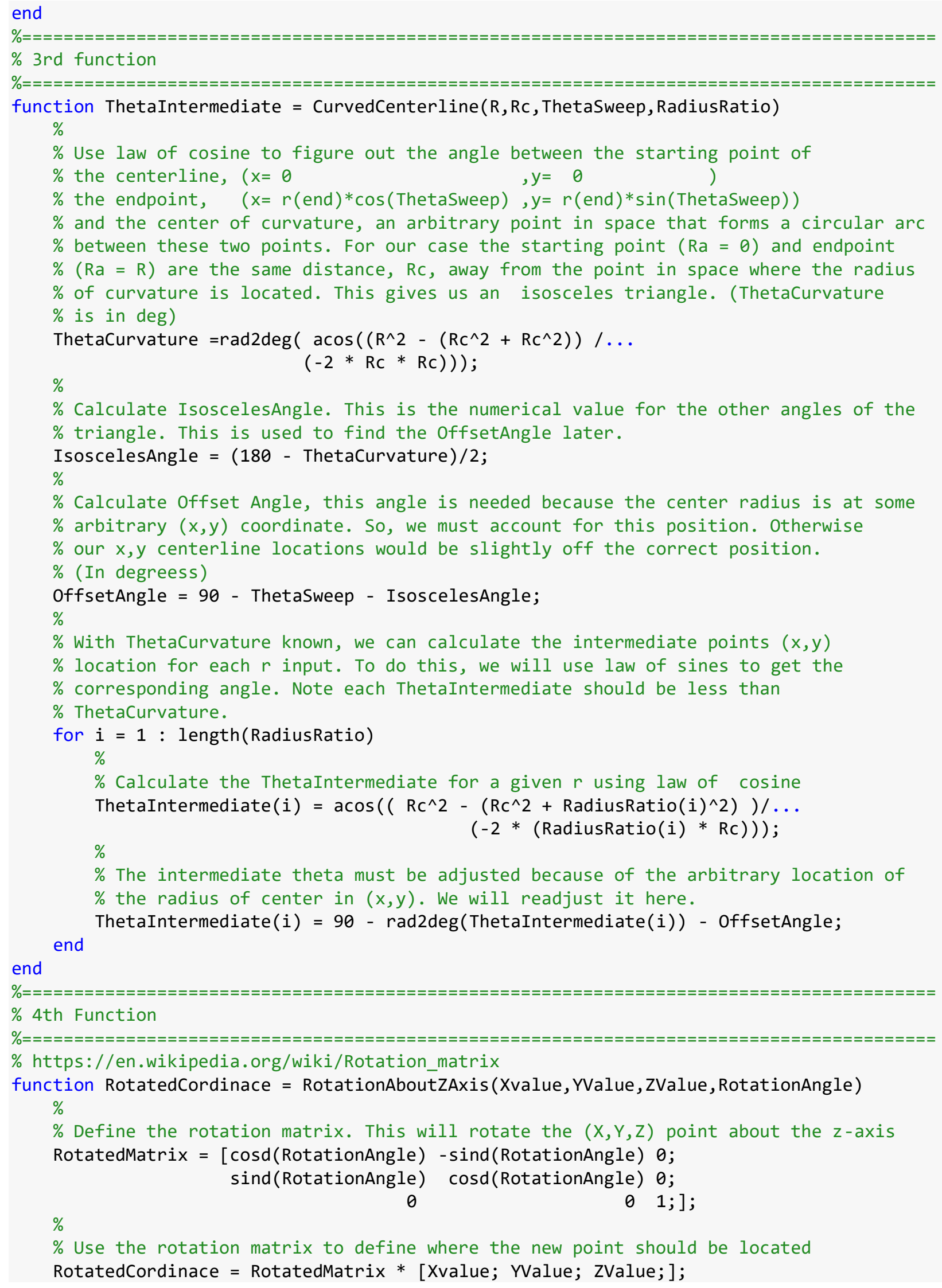




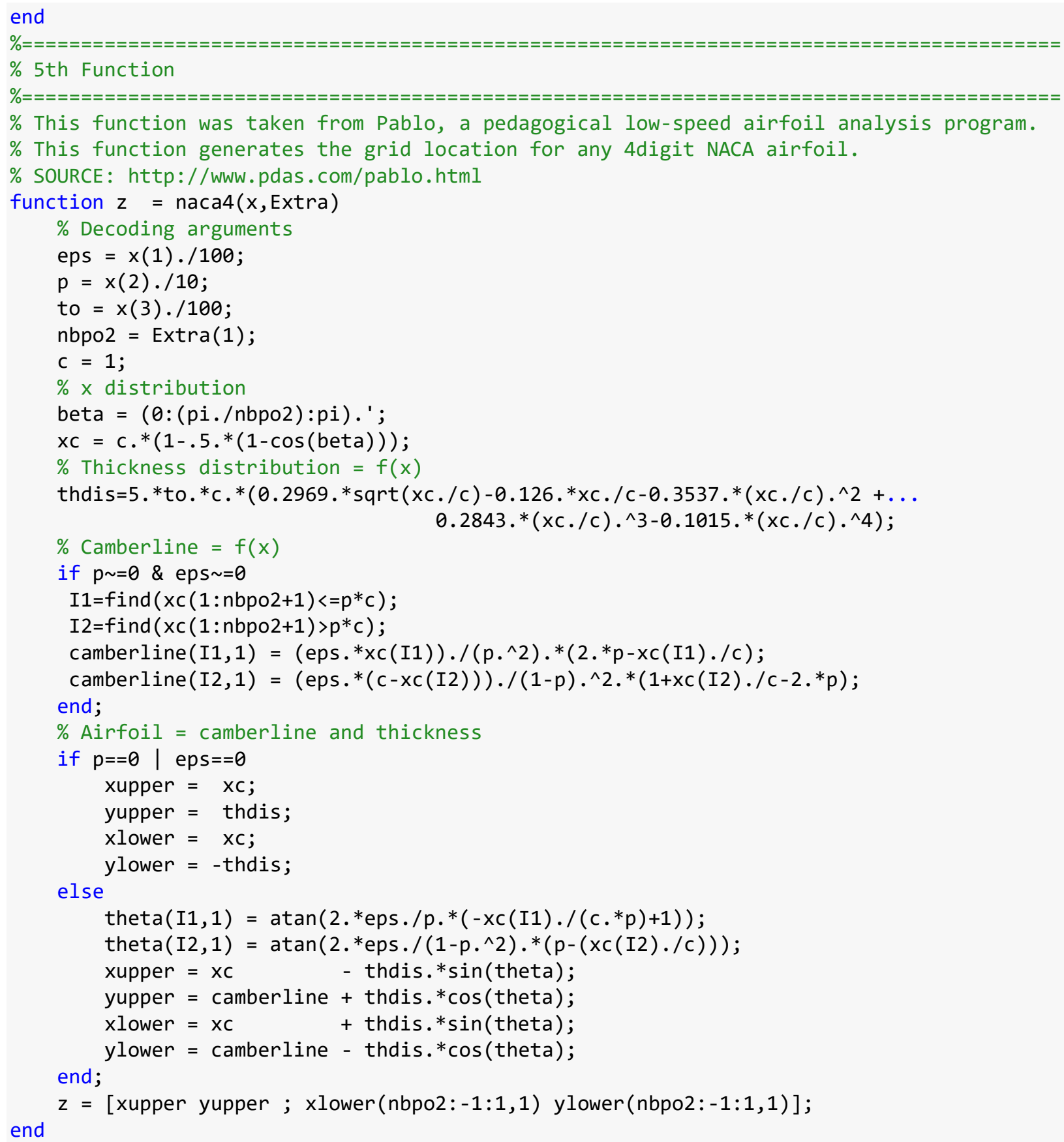




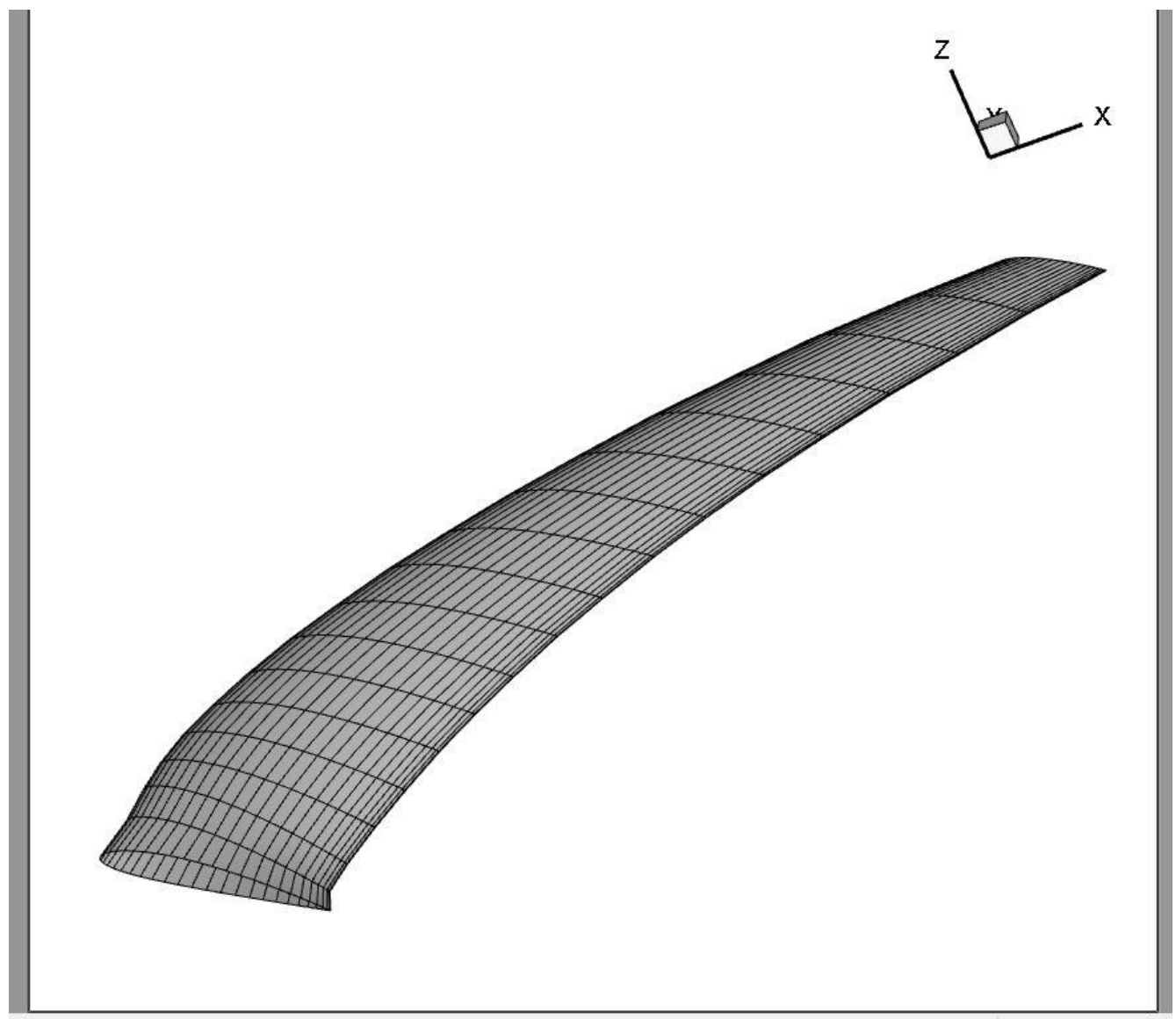

Figure 83: 'Geometry.dat' output from the MATLAB script imported into TECPLOT. 
APPENDIX B. PROPELLER DATASET

\begin{tabular}{|c|c|c|c|}
\hline$\frac{R_{a}}{R}$ & $\frac{c}{R}$ & $\beta$ & $\varphi_{\frac{R_{a}}{R}}$ \\
\hline 0.15 & 0.159 & 35.04 & 0 \\
\hline 0.2 & 0.145 & 41.53 & 0 \\
\hline 0.25 & 0.146 & 41.45 & 0 \\
\hline 0.3 & 0.151 & 37.33 & 0 \\
\hline 0.35 & 0.155 & 32.95 & 0 \\
\hline 0.4 & 0.158 & 29.67 & 0 \\
\hline 0.45 & 0.159 & 26.92 & 0 \\
\hline 0.5 & 0.159 & 24.5 & 0 \\
\hline 0.55 & 0.158 & 22.46 & 0 \\
\hline 0.6 & 0.156 & 20.65 & 0 \\
\hline 0.65 & 0.153 & 19 & 0 \\
\hline 0.7 & 0.148 & 17.5 & 0 \\
\hline 0.75 & 0.143 & 16.29 & 0 \\
\hline 0.8 & 0.136 & 15.19 & 0 \\
\hline 0.85 & 0.128 & 14.03 & 0 \\
\hline 0.9 & 0.119 & 12.94 & 0 \\
\hline 0.95 & 0.104 & 11.33 & 0 \\
\hline 1 & 0.09 & 9.7 & 0 \\
\hline
\end{tabular}

Table 11: The geometric properties used to define the straight propeller (18 stations defined)

\begin{tabular}{|c|c|c|c|}
\hline$\frac{R_{a}}{R}$ & $\frac{C}{R}$ & $\beta$ & $\varphi_{\frac{R_{a}}{R}}$ \\
\hline 0.15 & 0.159 & 35.04 & 0 \\
\hline 0.2 & 0.145 & 41.53 & 0.86699 \\
\hline 0.25 & 0.146 & 41.45 & 1.7352 \\
\hline 0.3 & 0.151 & 37.33 & 2.6045 \\
\hline 0.35 & 0.155 & 32.95 & 3.4749 \\
\hline 0.4 & 0.158 & 29.67 & 4.3468 \\
\hline 0.45 & 0.159 & 26.92 & 5.2203 \\
\hline 0.5 & 0.159 & 24.5 & 6.0956 \\
\hline 0.55 & 0.158 & 22.46 & 6.9729 \\
\hline 0.6 & 0.156 & 20.65 & 7.8526 \\
\hline 0.65 & 0.153 & 19 & 8.7347 \\
\hline 0.7 & 0.148 & 17.5 & 9.6196 \\
\hline 0.75 & 0.143 & 16.29 & 10.507 \\
\hline 0.8 & 0.136 & 15.19 & 11.398 \\
\hline 0.85 & 0.128 & 14.03 & 12.293 \\
\hline 0.9 & 0.119 & 12.94 & 13.191 \\
\hline 0.95 & 0.104 & 11.33 & 14.093 \\
\hline 1 & 0.09 & 9.7 & 15 \\
\hline
\end{tabular}

Table 12: The geometric properties used to define the swept-15 propeller (18 stations defined) 


\begin{tabular}{|c|c|c|c|}
\hline$\frac{R_{a}}{R}$ & $\frac{C}{R}$ & $\beta$ & $\varphi_{\frac{R_{a}}{R}}$ \\
\hline 0.15 & 0.159 & 35.04 & 0 \\
\hline 0.2 & 0.145 & 41.53 & 2.2359 \\
\hline 0.25 & 0.146 & 41.45 & 4.104 \\
\hline 0.3 & 0.151 & 37.33 & 5.9822 \\
\hline 0.35 & 0.155 & 32.95 & 7.8725 \\
\hline 0.4 & 0.158 & 29.67 & 9.7775 \\
\hline 0.45 & 0.159 & 26.92 & 11.699 \\
\hline 0.5 & 0.159 & 24.5 & 13.641 \\
\hline 0.55 & 0.158 & 22.46 & 15.606 \\
\hline 0.6 & 0.156 & 20.65 & 17.596 \\
\hline 0.65 & 0.153 & 19 & 19.616 \\
\hline 0.7 & 0.148 & 17.5 & 21.669 \\
\hline 0.75 & 0.143 & 16.29 & 23.761 \\
\hline 0.8 & 0.136 & 15.19 & 25.895 \\
\hline 0.85 & 0.128 & 14.03 & 28.079 \\
\hline 0.9 & 0.119 & 12.94 & 30.318 \\
\hline 0.95 & 0.104 & 11.33 & 32.622 \\
\hline 1 & 0.09 & 9.7 & 35 \\
\hline
\end{tabular}

Table 13: The geometric properties used to define the swept-35 propeller (18 stations defined)

\begin{tabular}{|c|c|c|c|}
\hline$\frac{R_{a}}{R}$ & $\frac{c}{R}$ & $\beta$ & $\varphi_{\frac{R_{a}}{R}}$ \\
\hline 0.15 & 0.159 & 35.04 & 0 \\
\hline 0.2 & 0.145 & 41.53 & 2.4669 \\
\hline 0.25 & 0.146 & 41.45 & 5.0672 \\
\hline 0.3 & 0.151 & 37.33 & 7.6947 \\
\hline 0.35 & 0.155 & 32.95 & 10.356 \\
\hline 0.4 & 0.158 & 29.67 & 13.059 \\
\hline 0.45 & 0.159 & 26.92 & 15.81 \\
\hline 0.5 & 0.159 & 24.5 & 18.621 \\
\hline 0.55 & 0.158 & 22.46 & 21.503 \\
\hline 0.6 & 0.156 & 20.65 & 24.468 \\
\hline 0.65 & 0.153 & 19 & 27.533 \\
\hline 0.7 & 0.148 & 17.5 & 30.719 \\
\hline 0.75 & 0.143 & 16.29 & 34.053 \\
\hline 0.8 & 0.136 & 15.19 & 37.571 \\
\hline 0.85 & 0.128 & 14.03 & 41.322 \\
\hline 0.9 & 0.119 & 12.94 & 45.383 \\
\hline 0.95 & 0.104 & 11.33 & 49.87 \\
\hline 1 & 0.09 & 9.7 & 55 \\
\hline
\end{tabular}

Table 14: The geometric properties used to define the swept-55 propeller (18 stations defined) 


\begin{tabular}{|c|c|c|c|}
\hline$\frac{R_{a}}{R}$ & $\frac{C}{R}$ & $\beta$ & $\varphi_{\frac{R_{a}}{R}}$ \\
\hline 0.154 & 0.1555 & 36.53 & 0 \\
\hline 0.17 & 0.1491 & 39.39 & 0 \\
\hline 0.199 & 0.1451 & 41.44 & 0 \\
\hline 0.235 & 0.145 & 41.97 & 0 \\
\hline 0.276 & 0.1484 & 39.64 & 0 \\
\hline 0.318 & 0.1526 & 35.62 & 0 \\
\hline 0.363 & 0.1559 & 32.04 & 0 \\
\hline 0.407 & 0.1583 & 29.27 & 0 \\
\hline 0.451 & 0.159 & 26.85 & 0 \\
\hline 0.495 & 0.159 & 24.72 & 0 \\
\hline 0.538 & 0.1583 & 22.93 & 0 \\
\hline 0.58 & 0.1569 & 21.36 & 0 \\
\hline 0.62 & 0.155 & 19.96 & 0 \\
\hline 0.659 & 0.1522 & 18.7 & 0 \\
\hline 0.697 & 0.1483 & 17.58 & 0 \\
\hline 0.732 & 0.1448 & 16.68 & 0 \\
\hline 0.766 & 0.1409 & 15.93 & 0 \\
\hline 0.798 & 0.1362 & 15.22 & 0 \\
\hline 0.828 & 0.1315 & 14.52 & 0 \\
\hline 0.856 & 0.127 & 13.89 & 0 \\
\hline 0.881 & 0.1227 & 13.36 & 0 \\
\hline 0.905 & 0.1178 & 12.81 & 0 \\
\hline 0.925 & 0.1119 & 12.17 & 0 \\
\hline 0.944 & 0.106 & 11.54 & 0 \\
\hline 0.959 & 0.101 & 11 & 0 \\
\hline 0.973 & 0.0973 & 10.59 & 0 \\
\hline 0.983 & 0.0946 & 10.29 & 0 \\
\hline 0.991 & 0.0927 & 10.06 & 0 \\
\hline 0.997 & 0.0914 & 9.89 & 0 \\
\hline 1 & 0.09 & 9.70 & 0 \\
\hline
\end{tabular}

Table 15: The geometric properties used to define the straight propeller (30 stations defined) 


\begin{tabular}{|c|c|c|c|}
\hline$\frac{R_{a}}{R}$ & $\frac{c}{R}$ & $\beta$ & $\varphi_{\frac{R_{a}}{R}}$ \\
\hline 0.154 & 0.1555 & 36.53 & 0.068905 \\
\hline 0.17 & 0.1491 & 39.39 & 0.34643 \\
\hline 0.199 & 0.1451 & 41.44 & 0.84963 \\
\hline 0.235 & 0.145 & 41.97 & 1.4747 \\
\hline 0.276 & 0.1484 & 39.64 & 2.1871 \\
\hline 0.318 & 0.1526 & 35.62 & 2.9177 \\
\hline 0.363 & 0.1559 & 32.04 & 3.7015 \\
\hline 0.407 & 0.1583 & 29.27 & 4.469 \\
\hline 0.451 & 0.159 & 26.85 & 5.2378 \\
\hline 0.495 & 0.159 & 24.72 & 6.008 \\
\hline 0.538 & 0.1583 & 22.93 & 6.7622 \\
\hline 0.58 & 0.1569 & 21.36 & 7.5004 \\
\hline 0.62 & 0.155 & 19.96 & 8.2051 \\
\hline 0.659 & 0.1522 & 18.7 & 8.8938 \\
\hline 0.697 & 0.1483 & 17.58 & 9.5664 \\
\hline 0.732 & 0.1448 & 16.68 & 10.187 \\
\hline 0.766 & 0.1409 & 15.93 & 10.792 \\
\hline 0.798 & 0.1362 & 15.22 & 11.363 \\
\hline 0.828 & 0.1315 & 14.52 & 11.899 \\
\hline 0.856 & 0.127 & 13.89 & 12.401 \\
\hline 0.881 & 0.1227 & 13.36 & 12.849 \\
\hline 0.905 & 0.1178 & 12.81 & 13.281 \\
\hline 0.925 & 0.1119 & 12.17 & 13.642 \\
\hline 0.944 & 0.106 & 11.54 & 13.985 \\
\hline 0.959 & 0.101 & 11 & 14.256 \\
\hline 0.973 & 0.0973 & 10.59 & 14.51 \\
\hline 0.983 & 0.0946 & 10.29 & 14.691 \\
\hline 0.991 & 0.0927 & 10.06 & 14.836 \\
\hline 0.997 & 0.0914 & 9.89 & 14.945 \\
\hline 1 & 0.09 & 9.70 & 15 \\
\hline
\end{tabular}

Table 16: The geometric properties used to define the swept-15 propeller (30 stations defined) 


\begin{tabular}{|c|c|c|c|}
\hline$\frac{R_{a}}{R}$ & $\frac{c}{R}$ & $\beta$ & $\varphi_{\frac{R_{a}}{R}}$ \\
\hline 0.154 & 0.1555 & 36.53 & 0.52425 \\
\hline 0.17 & 0.1491 & 39.39 & 1.119 \\
\hline 0.199 & 0.1451 & 41.44 & 2.1986 \\
\hline 0.235 & 0.145 & 41.97 & 3.5426 \\
\hline 0.276 & 0.1484 & 39.64 & 5.0793 \\
\hline 0.318 & 0.1526 & 35.62 & 6.6612 \\
\hline 0.363 & 0.1559 & 32.04 & 8.3663 \\
\hline 0.407 & 0.1583 & 29.27 & 10.045 \\
\hline 0.451 & 0.159 & 26.85 & 11.738 \\
\hline 0.495 & 0.159 & 24.72 & 13.446 \\
\hline 0.538 & 0.1583 & 22.93 & 15.132 \\
\hline 0.58 & 0.1569 & 21.36 & 16.797 \\
\hline 0.62 & 0.155 & 19.96 & 18.4 \\
\hline 0.659 & 0.1522 & 18.7 & 19.983 \\
\hline 0.697 & 0.1483 & 17.58 & 21.545 \\
\hline 0.732 & 0.1448 & 16.68 & 23.003 \\
\hline 0.766 & 0.1409 & 15.93 & 24.439 \\
\hline 0.798 & 0.1362 & 15.22 & 25.809 \\
\hline 0.828 & 0.1315 & 14.52 & 27.111 \\
\hline 0.856 & 0.127 & 13.89 & 28.344 \\
\hline 0.881 & 0.1227 & 13.36 & 29.46 \\
\hline 0.905 & 0.1178 & 12.81 & 30.545 \\
\hline 0.925 & 0.1119 & 12.17 & 31.461 \\
\hline 0.944 & 0.106 & 11.54 & 32.342 \\
\hline 0.959 & 0.101 & 11 & 33.044 \\
\hline 0.973 & 0.0973 & 10.59 & 33.706 \\
\hline 0.983 & 0.0946 & 10.29 & 34.182 \\
\hline 0.991 & 0.0927 & 10.06 & 34.566 \\
\hline 0.997 & 0.0914 & 9.89 & 34.855 \\
\hline 1 & 0.09 & 9.70 & 35 \\
\hline
\end{tabular}

Table 17: The geometric properties used to define the swept-35 propeller (30 stations defined) 


\begin{tabular}{|c|c|c|c|}
\hline$\frac{R_{a}}{R}$ & $\frac{C}{R}$ & $\beta$ & $\varphi_{\frac{R_{a}}{R}}$ \\
\hline 0.154 & 0.1555 & 36.53 & 0.093473 \\
\hline 0.17 & 0.1491 & 39.39 & 0.91726 \\
\hline 0.199 & 0.1451 & 41.44 & 2.4151 \\
\hline 0.235 & 0.145 & 41.97 & 4.2846 \\
\hline 0.276 & 0.1484 & 39.64 & 6.4297 \\
\hline 0.318 & 0.1526 & 35.62 & 8.6485 \\
\hline 0.363 & 0.1559 & 32.04 & 11.054 \\
\hline 0.407 & 0.1583 & 29.27 & 13.441 \\
\hline 0.451 & 0.159 & 26.85 & 15.866 \\
\hline 0.495 & 0.159 & 24.72 & 18.337 \\
\hline 0.538 & 0.1583 & 22.93 & 20.804 \\
\hline 0.58 & 0.1569 & 21.36 & 23.271 \\
\hline 0.62 & 0.155 & 19.96 & 25.681 \\
\hline 0.659 & 0.1522 & 18.7 & 28.097 \\
\hline 0.697 & 0.1483 & 17.58 & 30.524 \\
\hline 0.732 & 0.1448 & 16.68 & 32.834 \\
\hline 0.766 & 0.1409 & 15.93 & 35.157 \\
\hline 0.798 & 0.1362 & 15.22 & 37.426 \\
\hline 0.828 & 0.1315 & 14.52 & 39.639 \\
\hline 0.856 & 0.127 & 13.89 & 41.791 \\
\hline 0.881 & 0.1227 & 13.36 & 43.797 \\
\hline 0.905 & 0.1178 & 12.81 & 45.81 \\
\hline 0.925 & 0.1119 & 12.17 & 47.563 \\
\hline 0.944 & 0.106 & 11.54 & 49.303 \\
\hline 0.959 & 0.101 & 11 & 50.738 \\
\hline 0.973 & 0.0973 & 10.59 & 52.131 \\
\hline 0.983 & 0.0946 & 10.29 & 53.163 \\
\hline 0.991 & 0.0927 & 10.06 & 54.014 \\
\hline 0.997 & 0.0914 & 9.89 & 54.668 \\
\hline 1 & 0.09 & 9.70 & 55 \\
\hline
\end{tabular}

Table 18: The geometric properties used to define the swept-55 propeller (30 stations defined) 


\section{VITA}

Arthur David Wiedemann was born in Newport News, Virginia, in the year 1995. During his time in high school, he became interested in astronomy after watching the television series Cosmos: A Personal Voyage, presented by Carl Sagan. His interests would gradually shift to aerospace, which led him to pursue a B.S. degree in mechanical engineering at Old Dominion University. Due to his student internship at NASA Langley, he became interested in aeroacoustics and methods to reduce noise radiated from aircraft. This led to Arthur continuing his education and aquire an M.S. to conduct research of his own in the field of aeroacoustics. During Arthur's time as a graduate student at ODU, he became the head tutor for the Department of Mechanical and Aerospace Engineering where he helped both undergraduate and graduate students with their coursework. Arthur received his M.S. degree in Aerospace engineering in May 2020. 